U.S. DEPARTMENT OF THE INTERIOR

U.S. GEOLOGICAL SURVEY

Occurrence and Distribution of Pesticides and Volatile Organic Compounds in Ground Water and Surface Water in Central Arizona Basins, 1996-98, and Their Relation to Land Use

Water-Resources Investigations Report 01-4144

National Water-Quality Assessment Program 


\section{Occurrence and Distribution of Pesticides and Volatile Organic Compounds in Ground Water and Surface Water in Central Arizona Basins, 1996-98, and Their Relation to Land Use}

Water-Resources Investigations Report 01—4144

National Water-Quality Assessment Program 


\section{U.S. DEPARTMENT OF THE INTERIOR \\ GALE A. NORTON, Secretary}

U.S. GEOLOGICAL SURVEY

Charles G. Groat, Director

The use of firm, trade, and brand names in this report is for identification purposes only and does not constitute endorsement by the U.S. Geological Survey.

For additional information write to:

District Chief

U.S. Geological Survey

Water Resources Division

520 N. Park Avenue, Suite 221

Tucson, AZ 85719-5035
Copies of this report can be purchased from:

U.S. Geological Survey

Information Services

Box 25286

Federal Center

Denver, C0 80225-0046

Information regarding research and data-collection programs of the U.S. Geological Survey is available on the Internet via the World Wide Web. You may connect to the home page for the Arizona District Office using the URL http://az.water.usgs.gov. 


\section{FOREWORD}

The U.S. Geological Survey (USGS) is committed to serve the Nation with accurate and timely scientific information that helps enhance and protect the overall quality of life, and facilitates effective management of water, biological, energy, and mineral resources. Information on the quality of the Nation's water resources is of critical interest to the USGS because it is so integrally linked to the long-term availability of water that is clean and safe for drinking and recreation and that is suitable for industry, irrigation, and habitat for fish and wildlife. Escalating population growth and increasing demands for the multiple water uses make water availability, now measured in terms of quantity and quality, even more critical to the long-term sustainability of our communities and ecosystems.

The USGS implemented the National Water-Quality Assessment (NAWQA) Program to support national, regional, and local information needs and decisions related to water-quality management and policy. Shaped by and coordinated with ongoing efforts of other Federal, State, and local agencies, the NAWQA Program is designed to answer: What is the condition of our Nation's streams and ground water? How are the conditions changing over time? How do natural features and human activities affect the quality of streams and ground water, and where are those effects most pronounced? By combining information on water chemistry, physical characteristics, stream habitat, and aquatic life, the NAWQA Program aims to provide science-based insights for current and emerging water issues. NAWQA results can contribute to informed decisions that result in practical and effective water-resource management and strategies that protect and restore water quality.

Since 1991, the NAWQA Program has implemented interdisciplinary assessments in more than 50 of the Nation's most important river basins and aquifers, referred to as Study Units. Collectively, these Study Units account for more than 60 percent of the overall water use and population served by public water supply, and are representative of the Nation's major hydrologic landscapes, priority ecological resources, and agricultural, urban, and natural sources of contamination.

Each assessment is guided by a nationally consistent study design and methods of sampling and analysis. The assessments thereby build local knowledge about water-quality issues and trends in a particular stream or aquifer while providing an understanding of how and why water quality varies regionally and nationally. The consistent, multi-scale approach helps to determine if certain types of water-quality issues are isolated or pervasive, and allows direct comparisons of how human activities and natural processes affect water quality and ecological health in the Nation's diverse geographic and environmental settings. Comprehensive assessments on pesticides, nutrients, volatile organic compounds, trace metals, and aquatic ecology are developed at the national scale through comparative analysis of the Study-Unit findings.

The USGS places high value on the communication and dissemination of credible, timely, and relevant science so that the most recent and available knowledge about water resources can be applied in management and policy decisions. We hope this NAWQA publication will provide you the needed insights and information to meet your needs, and thereby foster increased awareness and involvement in the protection and restoration of our Nation's waters.

The NAWQA Program recognizes that a national assessment by a single program cannot address all waterresource issues of interest. External coordination at all levels is critical for a fully integrated understanding of watersheds and for cost-effective management, regulation, and conservation of our Nation's water resources. The program, therefore, depends extensively on the advice, cooperation, and information from other Federal, State, interstate, tribal, and local agencies, non-government organizations, industry, academia, and other stakeholder groups. The assistance and suggestions of all are greatly appreciated.

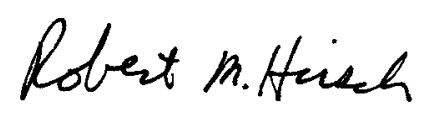

Robert M. Hirsch

Associate Director for Water 


\section{CONTENTS}

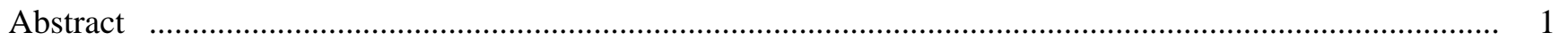

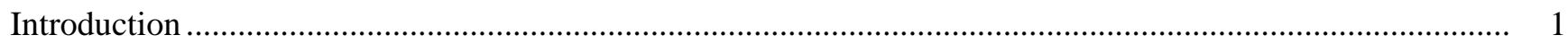

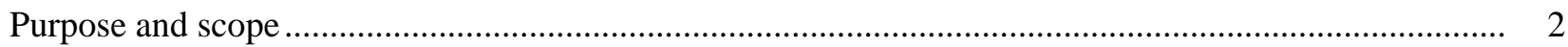

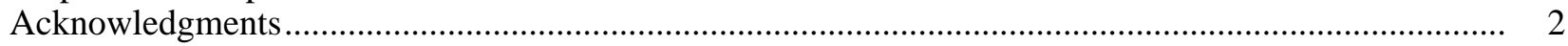

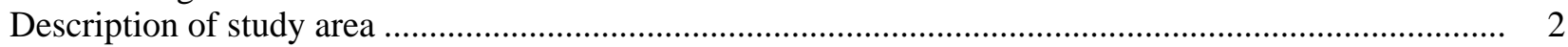

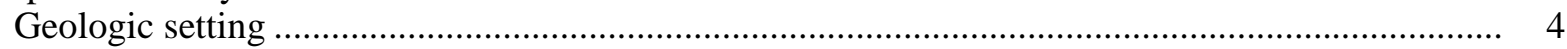

General surface-water and ground-water conditions ................................................................ 4

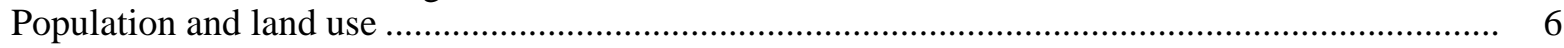

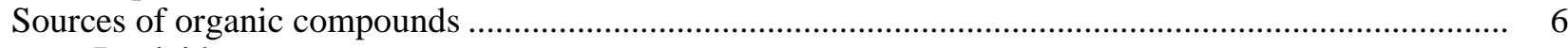

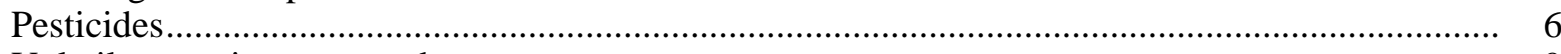

Volatile organic compounds

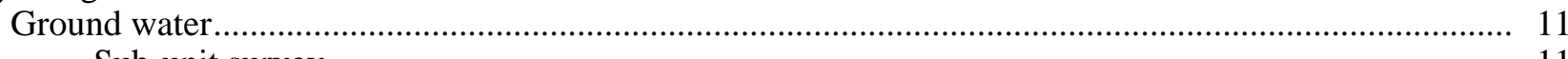

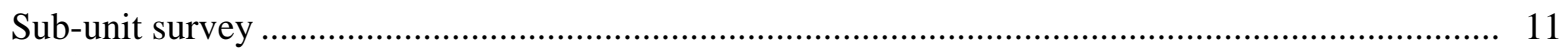

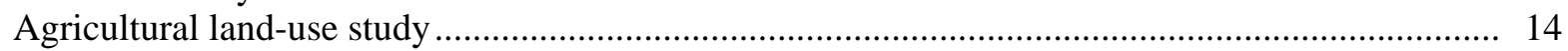

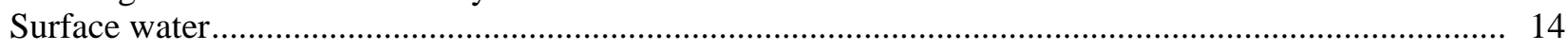

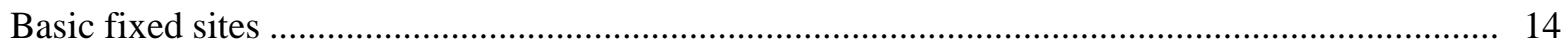

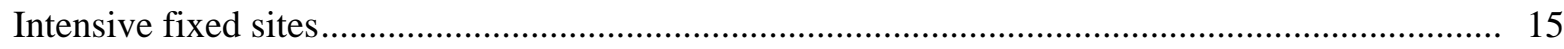

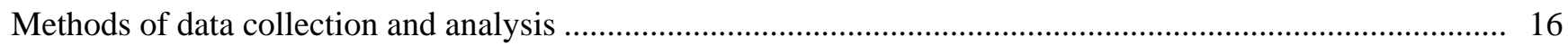

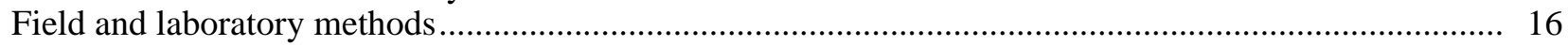

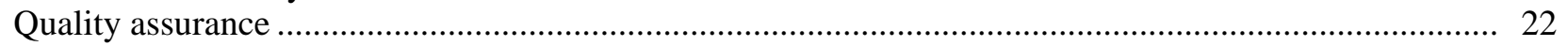

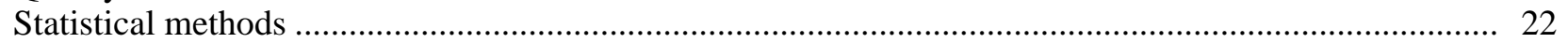

Occurrence and distribution of pesticides and volatile organic compounds in ground water and

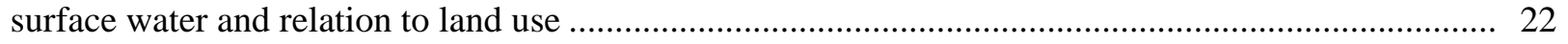

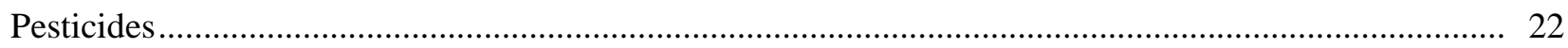

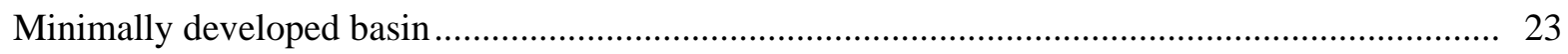

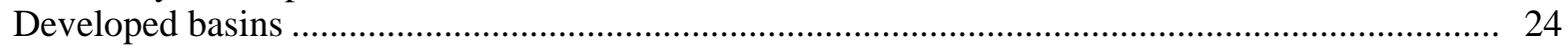

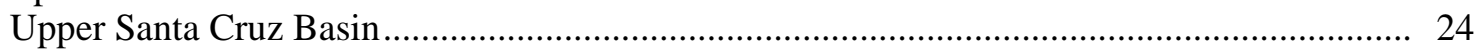

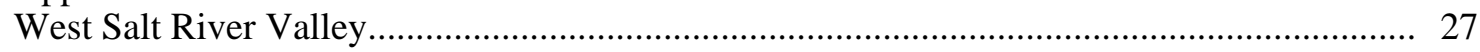

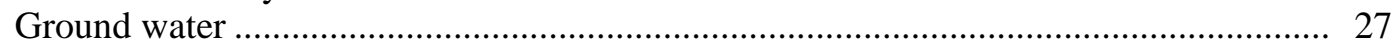

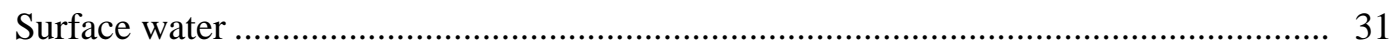

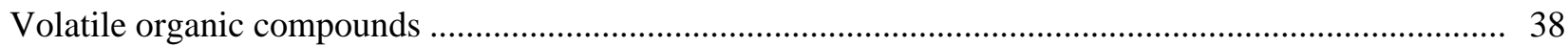

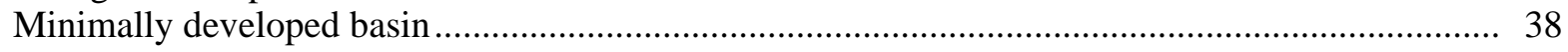

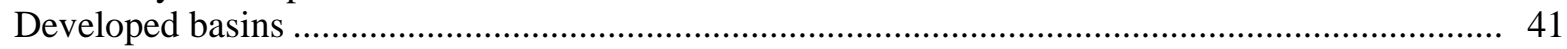

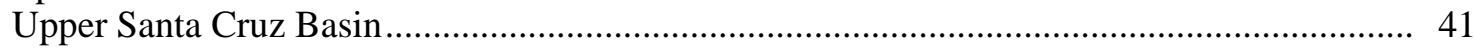

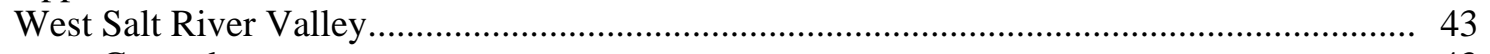

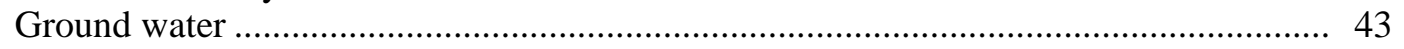

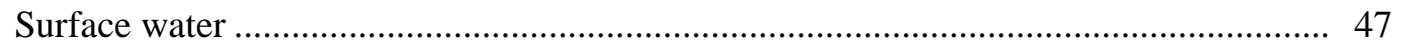

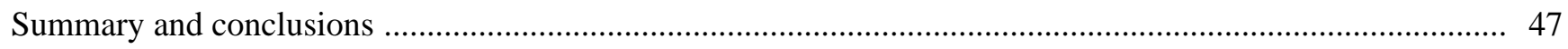

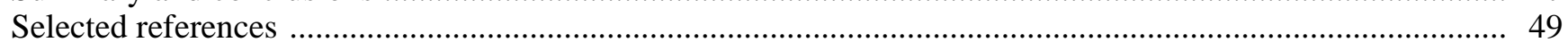

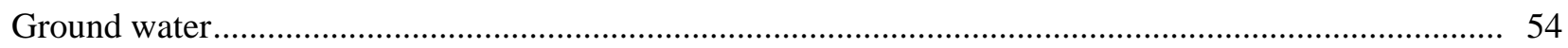

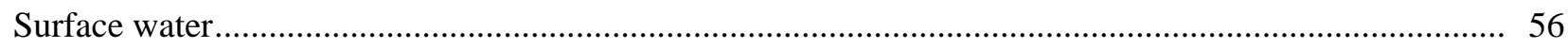

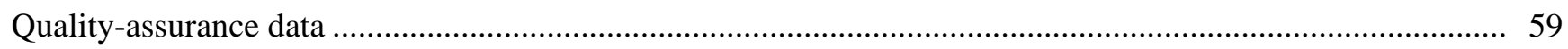

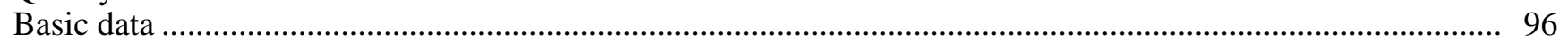




\section{FIGURES}

1. Map showing principal perennial and intermittent or ephemeral streams, and dams and selected canals, Central Arizona Basins study area.....

2. Chart showing surface-water sites, selected canals, wastewater-treatment plant outflows, interconnections with the Central Arizona Project Canal, agricultural return flows, and ground-water inputs to canals within the Central Arizona Basins study area .....

3. Map showing land use and land cover in the Central Arizona Basins study area.

4. Map showing surface-water sites and ground-water basins where samples were collected for pesticide and volatile organic compound analyses, Central Arizona Basins study area, 1996-98

5. Graph showing number of pesticide detections per well, Central Arizona Basins study area, 1996-98

6. Map showing locations of ground-water and surface-water sites where samples were collected for pesticide analyses, Upper Santa Cruz Basin, Central Arizona Basins study area.....

7. Map showing locations of ground-water and Surface-water sites where samples were collected for pesticide analyses, West Salt River Valley, Central Arizona Basins study area

8. Graph showing deethylatrazine/atrazine ratios for samples from wells in the agricultural land-use study area, Central Arizona Basins study area, August 1997 and February 1998

9. Graph showing selected pesticides detected in surface-water samples collected from the Gila River at Buckeye Canal, West Salt River Valley, Central Arizona Basins study area, 1996-98

10. Graph showing selected pesticides detected in surface-water samples collected from the Hassayampa River near Arlington, West Salt River Valley, Central Arizona Basins study area, 1996-98

11. Map showing locations of ground-water sites where samples were collected for volatile organic compound analyses, Sierra Vista subbasin, Central Arizona Basins study area, 1996-97

12. Graph showing number of volatile organic compound detections per well, Central Arizona Basins study area, 1996-98

13. Map showing locations of ground-water and surface-water sites where samples were collected for volatile organic compound analyses, Upper Santa Cruz River Basin, Central Arizona Basins study area

14. Map showing locations of ground-water and surface-water sites where samples were collected for volatile organic compound analyses, West Salt River Valley, Central Arizona Basins study area 


\section{TABLES}

Page

1. Recommended insecticides, herbicides, and defoliants for cotton crops in Arizona, 1940-87 .......... 9

2. Pesticides applied in the ground-water study areas, Central Arizona Basins study area, 1996-98 _.... 10

3. Study areas where ground-water samples were collected for pesticide and (or) volatile organic compound analyses, Central Arizona Basins study area, 1996-98

4. Surface-water sites where samples were collected for pesticide and (or) volatile organic compound analyses, Central Arizona Basins study area, 1996-98.

5. Pesticide and pesticide-degradation compound analyses by the National Water Quality Laboratory for surface-water and ground-water samples, Central Arizona Basins study area, 1996-98

6. Pesticide-degradation compound analyses by the U.S. Geological Survey, Organic Geochemistry Research Group, Lawrence, Kansas, for ground-water and surfacewater samples, Central Arizona Basins study area, 1996-98.

7. Volatile organic compound analyses by the National Water Quality Laboratory for ground-water and surface-water samples, Central Arizona Basins study area, 1996-98

8. Number of pesticides detected in ground-water and surface-water samples, Sierra Vista subbasin, Central Arizona Basins study area, 1996-97

9. Maximum Contaminant Levels, human health advisory limits, aquifer water-quality standards, and surface-water standards for pesticides detected in ground-water and surface-water samples, Central Arizona Basins study area, 1996-98.....

10. Number of pesticides detected in ground-water and surface-water samples in the Upper Santa Cruz Basin, Central Arizona Basins study area, 1996-98.

11. Number of pesticides detected in ground-water and surface-water samples,

West Salt River Valley, Central Arizona Basins study area, 1996-98

12. Number of volatile organic compounds detected in ground-water samples in the Sierra Vista subbasin, Central Arizona Basins study area, 1996-97

13. Maximum Contaminant Levels, human health advisory limits, aquifer water-quality standards, and surface-water standards for volatile organic compounds detected in ground-water and surface-water samples, Central Arizona Basins study area, 1996-98

14. Number of volatile organic compounds detected in ground-water and surface-water samples, Upper Santa Cruz Basin, Central Arizona Basins study area, 1996-98

15. Number of volatile organic compounds detected in ground-water and surface-water samples, West Salt River Valley, Central Arizona Basins study area, 1996-98.

16. Volatile organic compounds in water from three wells having the highest number of detections, West Salt River Valley, Central Arizona Basins study area, 1996-98

17. Quality-control samples collected in the Central Arizona Basins study area, 1996-98

18. Pesticide and pesticide-degradation compounds detected in field and equipment blanks for ground-water samples, Central Arizona Basins study area, 1996-98

19. Volatile organic compounds detected in field blanks for ground-water samples collected March 7 to September 17, 1996, Central Arizona Basins study area. 
20. Volatile organic compounds detected in field blanks, source-solution blanks, and equipment blanks for ground-water samples collected October 1, 1996, to

July 9, 1998, Central Arizona Basins study area.....

21. Volatile organic compounds detected in trip blanks and polyvinyl chloride wash samples for ground-water samples, Central Arizona Basins study area, 1996-98

22. Percent recovery statistics for pesticides and pesticide-degradation compounds detected in field-matrix and laboratory-spike samples for ground-water samples and in laboratory set-spike samples, Central Arizona Basins study area, 1996-98

23. Percent recovery statistics for surrogate compounds for pesticides and volatile organic compounds detected in ground-water and surface-water samples, Central Arizona Basins study area, 1996-98

24. Percent recovery statistics for volatile organic compounds detected in field-matrix spike samples for ground-water and surface-water samples, Central Arizona Basins study area, 1996-98

25. Pesticide and pesticide-degradation compounds detected in field blanks for surface-water samples, Central Arizona Basins study area, 1996-98

26. Volatile organic compounds detected in field blanks, source-solution blanks, equipment blanks, trip blanks, and split replicates collected at 91st Avenue wastewater-treatment plant outfall near Phoenix, 1996-97

27. Summary statistics for pesticides and pesticide-degradation compounds detected in field-matrix spike samples and replicates for surface-water samples, Central Arizona Basins study area, 1996-98

28. Results of pesticide and pesticide-degradation compound analyses of concurrent replicates for surface-water samples, Central Arizona Basins study area, 1996-98

29. Pesticide and pesticide-degradation compounds detected in split-replicates for surface-water samples, Central Arizona Basins study area, 1996-98

30 Data for volatile organic compounds detected in ground-water samples collected April 1996 through September 1996, Central Arizona Basins study area

31. Site information for wells sampled, Central Arizona Basins study area, 1996-98 101

\section{CONVERSION FACTORS}

\begin{tabular}{rll}
\hline Multiply & \multicolumn{1}{c}{ By } & To obtain \\
foot $(\mathrm{ft})$ & 0.3048 & meter \\
mile $(\mathrm{mi})$ & 1.609 & kilometer \\
square mile $\left(\mathrm{mi}^{2}\right)$ & 2.590 & square kilometer \\
gallon $(\mathrm{gal})$ & 0.003785 & cubic meter \\
million gallons (Mgal) & 3,785 & cubic meter \\
\hline
\end{tabular}

\section{ABBREVIATED WATER-QUALITY UNITS}

Chemical concentrations are given only in metric units. Chemical concentration in water is given in milligrams per liter (mg/L) or micrograms per liter $(\mu \mathrm{g} / \mathrm{L})$. Milligrams per liter is a unit expressing the solute mass (milligrams) per unit volume (liter) of water. One thousand micrograms per liter is equivalent to 1 milligram per liter. For concentrations less than 7,000 milligrams per liter, the numerical value is about the same as for concentrations in parts per million. 
VERTICAL DATUM

Sea level: In this report, "sea level" refers to the National Geodetic Vertical Datum of 1929 (NGVD of 1929) — a geodetic datum derived from a general adjustment of the first-order level nets of both the United States and Canada, formerly called "Sea Level Datum of 1929".

\section{DEFINITION OF ABBREVIATIONS AND ACRONYMS}

$\begin{aligned} \text { ADEQ } & \text { Arizona Department of Environmental Quality } \\ \text { CAP } & \text { Central Arizona Project } \\ \text { CAZB } & \text { Central Arizona Basins } \\ \text { MCL } & \text { Maximum Contaminant Level } \\ \text { MRL } & \text { Minimum Reporting Level } \\ \text { DDE } & \text { Dichlorodiphenyldichloroethene } \\ \text { DDT } & \text { Dichlorodiphenyldichloroethane } \\ \text { GCMS } & \text { Gas chromatograph/mass spectrometry with selected ion monitoring } \\ \text { HPLC } & \text { High-performance liquid chromatography } \\ \text { LUS } & \text { Land-use study } \\ \text { NAWQA } & \text { National Water-Quality Assessment } \\ \text { NWQL } & \text { National Water-Quality Laboratory } \\ \text { PCE } & \text { Tetrachloroethene } \\ \text { SUS } & \text { Sub-unit survey } \\ \text { TCE } & \text { Trichloroethene } \\ \text { TRI } & \text { Toxics Release Inventory } \\ \text { USEPA } & \text { U.S. Environmental Protection Agency } \\ \text { USGS } & \text { U.S. Geological Survey } \\ \text { VOC } & \text { Volatile organic compound } \\ \text { WWTP } & \text { Wastewater-Treatment Plant }\end{aligned}$

\section{WELL-NUMBERING SYSTEM, ARIZONA}

The well numbers used by the U.S. Geological Survey in Arizona are in accordance with the Bureau of Land Management's system of land subdivision. The land survey in Arizona is based on the Gila and Salt River Meridian and Base Line, which divide the State into four quadrants. These quadrants are designated counterclockwise by the capital letters, A,B, C, and D. All land north and east of the point of origin is in A quadrant, that north and west in B quadrant, that south and west in C quadrant, and that south and east in D quadrant. The first digit of a well number indicates the township; the second, the range; and the third, the section in which the well is situated. The lowercase letters $a, b, c$, and $d$ after the section number indicate the well location within the section. The first letter denotes a particular 160-acre tract; the second, the 40-acre tract; and the third, the 10 -acre tract. These letters also are assigned in a counterclockwise direction, beginning in the northeast quarter. If the location is known within the 10-acre tract, three lowercase letters are shown in the well number. In the example shown, well number (A01-01)22dcc designated the well as being in the SW1/4SW1/4SE1/4, sec. 22 T. 1 N., R. 1 E. Where more than 1 well is within a 10-acre tract, consecutive numbers beginning with 1 are added as suffixes. 
WELL (A-01-01)22dcc

R. $1 \mathrm{E}$.

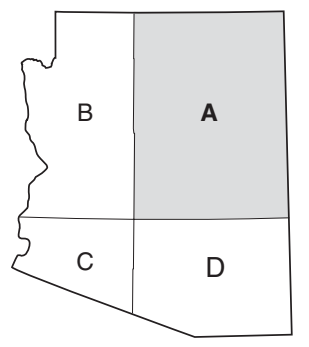

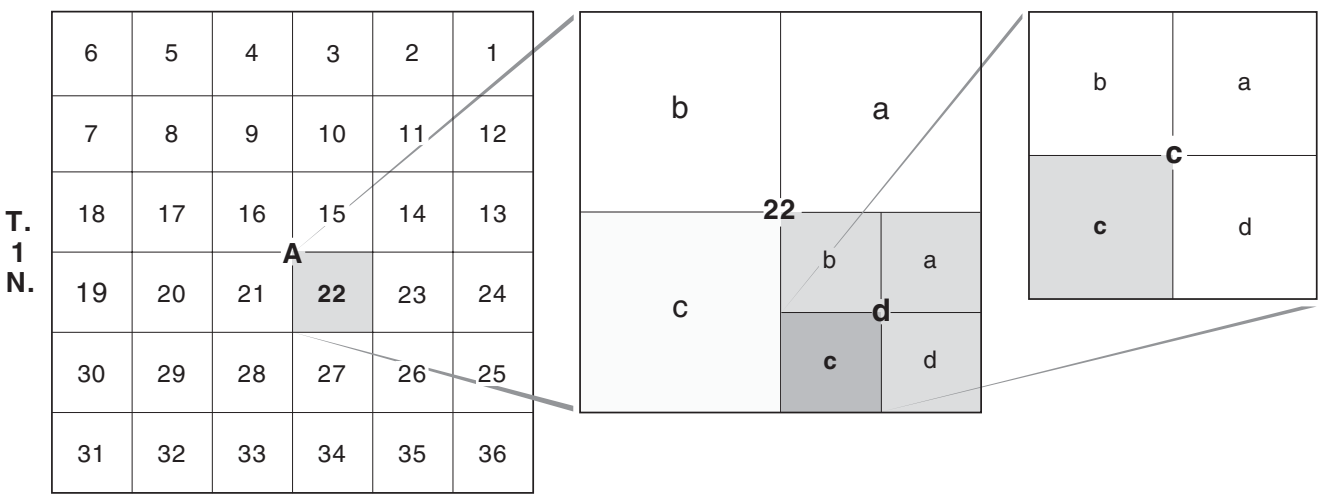




\title{
Occurrence and Distribution of Pesticides and Volatile Organic Compounds in Ground Water and Surface Water in Central Arizona Basins, 1996-98, and Their Relation to Land Use
}

\author{
By D.J. Gellenbeck and D.W. Anning
}

\begin{abstract}
Samples of ground water and surface water from the Sierra Vista subbasin, the Upper Santa Cruz Basin, and the West Salt River Valley were collected and analyzed to determine the occurrence and distribution of pesticides and volatile organic compounds in central Arizona. The study was done during 1996-98 within the Central Arizona Basins study unit of the National Water-Quality Assessment program. This study included 121 wells and 4 surface-water sites in the 3 basins and the analyses of samples from 4 sites along the Santa Cruz River that were part of a separate study. Samples were collected from 121 wells and 3 surface-water sites for pesticide analyses, and samples were collected from 109 wells and 3 surface-water sites for volatile organic compound analyses.

Certain pesticides detected in ground water and surface water can be related specifically to agricultural or urban uses; others can be related to multiple land uses. Effects from historical agriculture are made evident by detections of DDE in ground-water and surface-water samples collected in the West Salt River Valley and detections of atrazine and deethylatrazine in the ground water in the Upper Santa Cruz Basin. Effects from present agriculture are evident in the seasonal variability in concentrations of pre-emergent pesticides in surface-water samples from the West Salt River Valley. Several detections of DDE and dieldrin in surface water were higher than established water-quality limits. Effects of urban land use are made evident by detections of volatile organic compounds in ground water and surface water from the West Salt River Valley. Detections of volatile organic compounds in surface water from the Santa Cruz River near Nogales, Arizona, also are indications of the effects of urban land use. One detection of tetrachloroethene in ground water was higher than established water-quality limits.

Water reuse is an important conservation technique in the Southwest; however, the reuse of water provides a transport mechanism for pesticides and volatile organic compounds to reach areas that are not normally affected by manmade compounds from specific land-use activities. The most complex mixture of pesticides and volatile organic compounds is in the West Salt River Valley and is the result of watermanagement practices and the combination of land uses in this basin throughout history.
\end{abstract}

\section{INTRODUCTION}

Predicted population growth in Arizona will result in an increased demand for good-quality drinking water. Between 1980 and 1990, the population in Arizona increased by 35 percent; and between 1990 and 2000, the population increased by another 40 percent (Arizona Department of Economic Security, rev. March 27, 2001). Most population growth was in and near the metropolitan areas of Phoenix and Tucson (Cordy and others, 1998). As the demand for goodquality drinking water increases in these metropolitan 
areas, knowledge about the occurrence and distribution of organic compounds in the water resources becomes more important. To date (2000), there have been few studies that completed an assessment of these compounds on a large scale in Arizona.

The National Water-Quality Assessment (NAWQA) program begun by the U.S. Geological Survey (USGS) provided the opportunity to assess the occurrence and distribution of organic and inorganic compounds in the water resources of central Arizona during 1996-98. The objectives of the NAWQA program are to describe the current water-quality conditions and trends in rivers, streams, and ground water and to understand the natural and human factors that affect the conditions and trends in water quality throughout the Nation (Hirsch and others, 1988). The Central Arizona Basins (CAZB) study area (fig. 1), which includes $34,700 \mathrm{mi}^{2}$, was one of more than 50 study areas across the United States that were selected to help meet these objectives. Surface-water and ground-water samples from the CAZB study area were analyzed to determine the occurrence and distribution of pesticides and volatile organic compounds (VOCs) in south-central Arizona during 1996-98.

\section{Purpose and Scope}

This report presents analytical results for pesticides and VOCs in ground water and surface water in the southern and central parts of the CAZB study area during 1996-98. In this report, the term "pesticides" refers to pesticide parent and degradation compounds. Activities in urban and agricultural land-use areas are evaluated as possible sources of pesticides and VOCs detected in the water samples. During 1996-98, ground-water samples were collected from three basins - the West Salt River Valley, the Sierra Vista subbasin, and the Upper Santa Cruz Basin. Samples for pesticide analyses were collected at 121 wells, and samples for VOC analyses were collected at 109 wells. Surface-water samples primarily were collected from four sites in southern and central Arizona; at one site, alternate sampling locations were used depending on flow conditions. During 1996-98, 77 samples for pesticide analyses were collected at 3 of the 4 surfacewater sites, and 13 samples for VOC analyses were collected at 3 of the 4 surface-water sites. Comparisons of occurrence and distribution of pesticides and VOCs among the ground-water basins and seasonal variability of pesticide occurrence and distribution at some surface-water sites are included in this report.

Quality-control samples were collected to evaluate the quality of the environmental data. During 1996-98, 12 different types of quality-control samples were collected to evaluate contamination, bias, and variability in the ground-water and surface-water data. The discussion of these results is included in the section entitled "Quality-Assurance Information."

\section{Acknowledgments}

Henry Sanger, Alissa Coes, Dave Peyton, Jack Edmonds, Julie Rees, Karen Beaulieu, Joe Capesius, Laurie Wirt, Christie O’Day, Ann Tillery, Ken Galyean, Rodrigo Morales, David Graham, and Gail Cordy (USGS) all participated in collection of ground-water and surface-water samples. Todd Ingersoll, Ray Davis, Melissa Butler, and Dawn McDoniel (USGS) processed many of the samples for pesticide analyses in the office laboratory. Alice Konieczki (USGS) collected samples on the Santa Cruz River; without these data, no information would be available about pesticides and VOCs in surface water from the Upper Santa Cruz Basin. Many of these people donated several hours of their personal time to ensure the accuracy and completeness of the data presented.

Ken Agnew, University of Arizona, Pesticide and Information Office, provided information for pesticides used in the study area. Private land owners allowed USGS personnel to measure water levels, sample ground water, drill monitoring wells, and sample surface water on their property. Many municipal agencies permitted sampling of their wells and allowed access to well records.

\section{Description of Study Area}

The CAZB study area has been divided into two hydrologic provinces - the Basin and Range Lowlands and the Central Highlands - on the basis of physiographic and hydrologic characteristics (Fenneman, 1931). The data discussed in this report are from the Basin and Range Lowlands Province of the CAZB study area, which includes about $19,000 \mathrm{mi}^{2}$ of southern and central Arizona. Altitudes range from about $800 \mathrm{ft}$ above sea level west of Phoenix near the study-area boundary to about $9,470 \mathrm{ft}$ southeast of Sierra Vista (Cordy and others, 1998). 


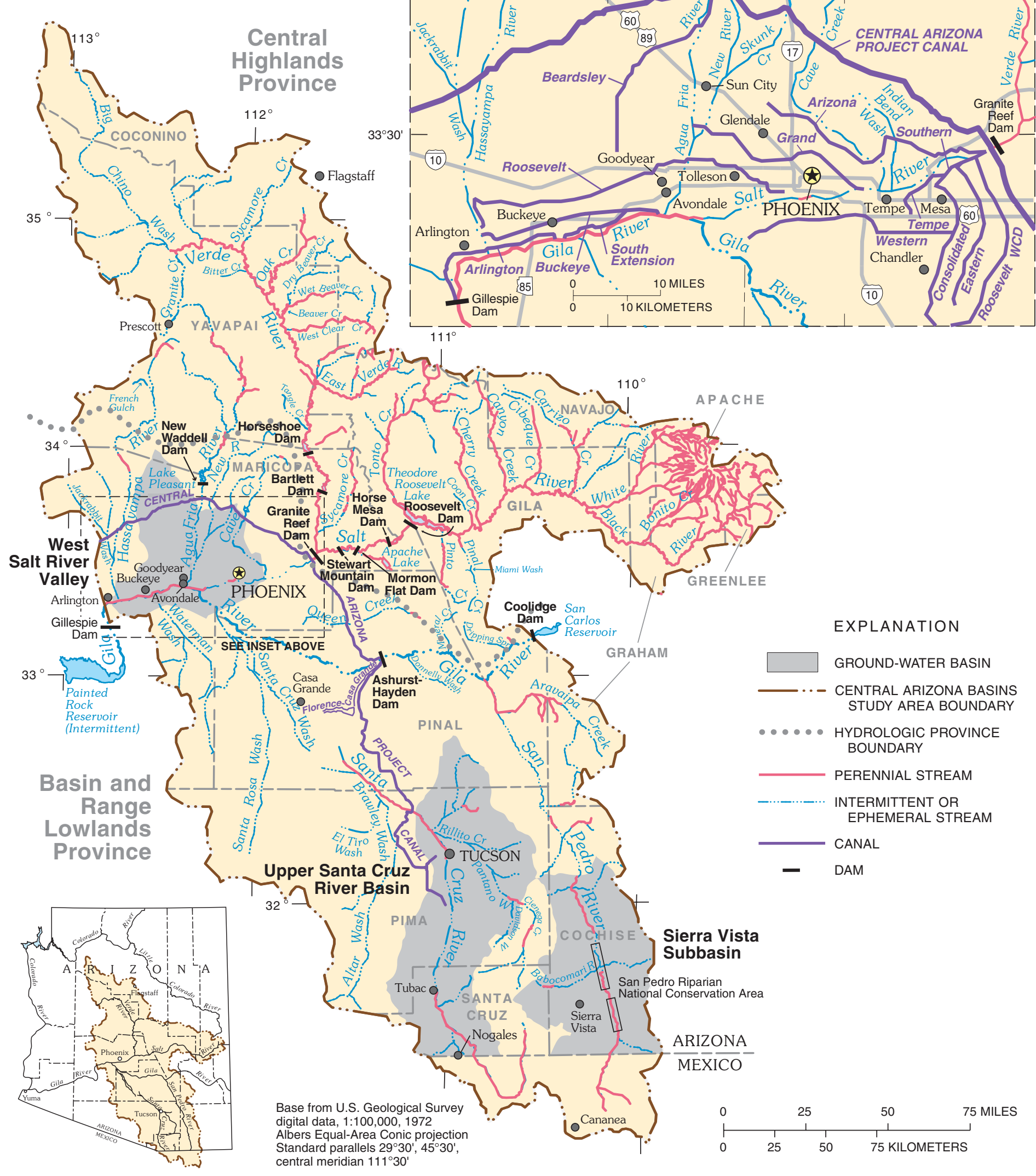

Figure 1. Principal perennial and intermittent or ephemeral streams, and dams and selected canals, Central Arizona Basins study area (perennial reaches from Arizona Game and Fish Department, 1993; digital data modified from U.S. Geological Survey, 1974-86; urban digital data for 1990 from Maricopa Association of Governments, Pima County, and the University of Arizona). 


\section{Geologic Setting}

The present-day geologic setting in the southern and central parts of the CAZB study area is in part the result of normal faulting during the Basin and Range Disturbance (Damon and others, 1984; Shafiqullah and others, 1980) that resulted in uplifted and downdropped blocks that form the mountains and valleys, respectively. Bedrock in the mountains includes Precambrian metamorphic and granitic rocks; Paleozoic sandstone, limestone, and shale; Mesozoic granitic, volcanic, and sedimentary rocks; and Cenozoic granitic, volcanic, and sedimentary rocks (Reynolds, 1988).

In the Basin and Range Lowlands, sediments were eroded from the surrounding uplifted mountains and deposited in the valleys in each basin during a period of internal drainage. The basin-fill deposits may be as much as $12,000 \mathrm{ft}$ thick (Cordy and others, 1998). Anderson and others (1992) identified these deposits as weakly to highly consolidated gravel, sand, silt, and clay that range in age from late Tertiary to Quaternary. Evaporite and mudstone deposits are found within the lower part of the basin-fill deposits. Stream alluvium overlies the basin-fill deposits and is present along the surface-water drainages in most of the alluvial basins.

\section{General Surface-Water and Ground-Water Conditions}

In the CAZB study area, the hydrologic system in the Basin and Range Lowlands is dominated by the regulation of streamflow and large amounts of groundwater pumping. Reservoirs and diversions on the Salt, Verde, and Agua Fria Rivers regulate surface water that contributes flow to the Gila River from the Central Highlands (fig. 1). Within the Basin and Range Lowlands, almost all flow in the Gila River is diverted at Ashurst-Hayden Dam into the Florence-Casa Grande Canal (fig. 1). Between this diversion and the metropolitan area of Phoenix, the flow in the Gila River is ephemeral, and the sources of streamflow are mainly irrigation-return flow and treated-sewage effluent.

The San Pedro and Santa Cruz Rivers in the Basin and Range Lowlands flow from Mexico northward into Arizona and contribute flow to the Gila River before the Gila River enters the metropolitan area of Phoenix. The headwaters of the San Pedro River are in Mexico, and flow is perennial in some reaches and intermittent in others (fig. 1). The headwaters of the Santa Cruz River are in Arizona, and a perennial reach of the river flows southward into Mexico and then northward back into Arizona. In Arizona, the Santa Cruz River contains perennial and ephemeral reaches. Treated-sewage effluent from wastewater-treatment plants (WWTPs) on the Santa Cruz River provide base flow between Nogales and Tubac and near Tucson. The channel of the Santa Cruz River becomes a network of distributary channels that are ephemeral between Tucson and the confluence with the Gila River. These distributary channels create a disconnection of the river. The Santa Cruz River contains irrigation-return flow in this reach.

Within the metropolitan area of Phoenix, downstream from canal diversions, and upstream from the confluence of the Salt and Gila Rivers, the primary source of water in the Salt River is outflow from the WWTPs at 23rd Avenue and 91st Avenue. These WWTPs process 71.3 billion gallons of sewage annually from the metropolitan Phoenix area (City of Phoenix, 1999). Seasonal floods and irrigation-return flow also can contribute to this reach. Downstream from the confluence of the Gila and Agua Fria Rivers, most of the surface water is diverted into the Buckeye Canal operated by the Buckeye Irrigation District (fig. 2). Diverted surface water in this canal, as well as other canals in the metropolitan area of Phoenix, is supplemented with ground water.

Farther downstream, irrigation-return flows contribute to the surface water in the Gila River. The Hassayampa River primarily is ephemeral; irrigationreturn flow is the source of water in localized reaches and enters the Gila River at the confluence.

Ground water in the Basin and Range Lowlands primarily is in the stream alluvium and basin-fill deposits. The most productive aquifers are the thick basin-fill deposits. Most water is obtained from the upper 1,000 ft of these deposits and generally is under unconfined conditions (Anderson and others, 1992). Primary locations of ground-water recharge are along mountain fronts and along the axes of structural basins where major streams and rivers contain flow for long periods. Irrigation water applied at amounts that exceed crop requirements provides recharge to the underlying aquifer in areas of active cultivation and in urban areas where landscapes are overwatered. Seepage from unlined irrigation canals also can provide recharge to aquifers. 


\section{Upstream End (Coolidge Dam)}

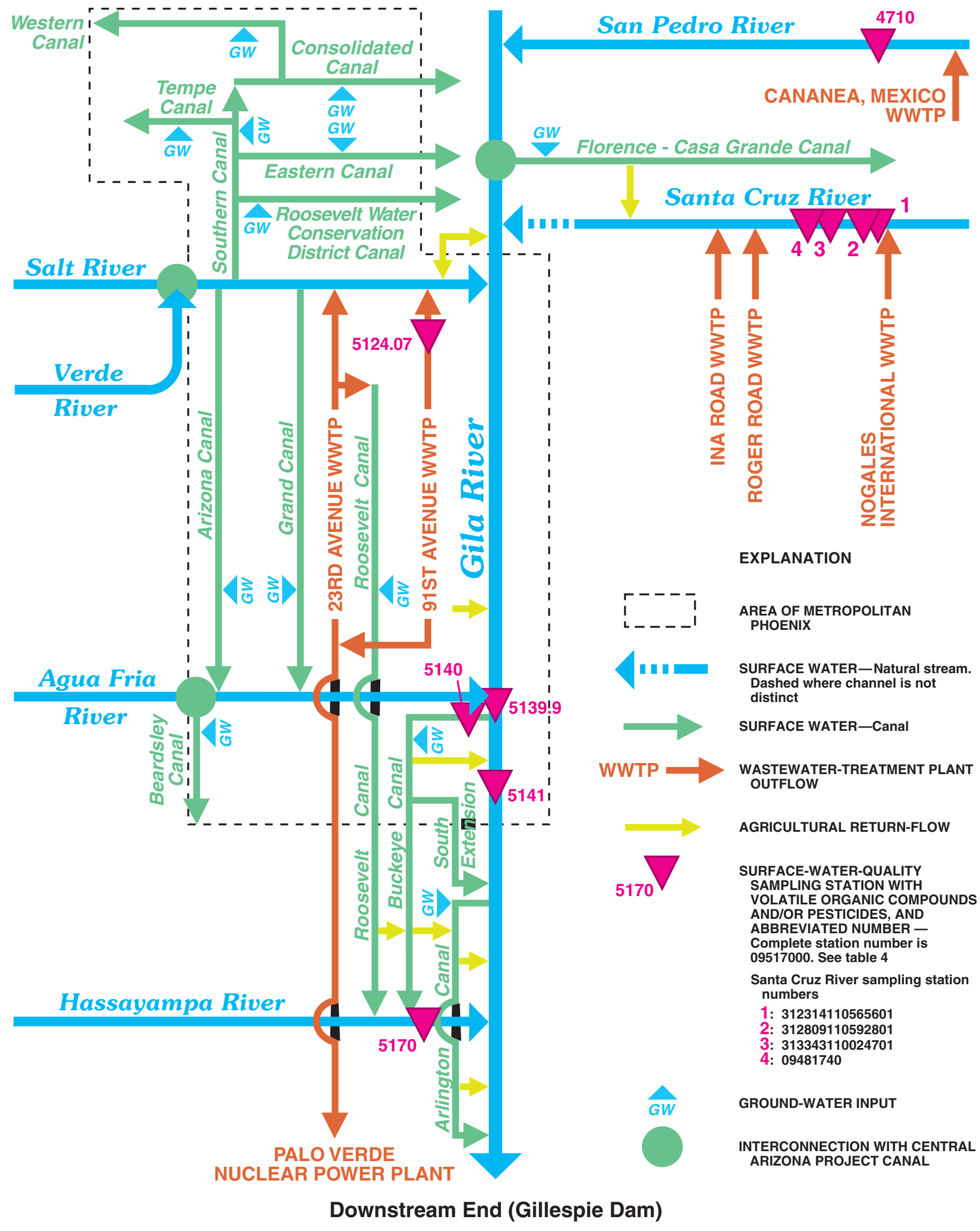

Figure 2. Surface-water sites, selected canals, wastewater-treatment plant outflows, interconnections with the Central Arizona Project Canal, agricultural return flows, and ground-water inputs to canals within the Central Arizona Basins study area. 
Natural ground-water movement generally parallels surface-water movement within each basin; ground water moves from the mountains surrounding the basin toward the stream and then along the structural axis of the basin. Depth to ground water ranges from zero near perennial streams and some irrigated areas to as much as 1,300 ft below land surface (Cordy and others, 1998). In some basins, water levels have declined as much as $400 \mathrm{ft}$ (Anderson and others, 1992, pl. 2). In the metropolitan area of Phoenix (Thomsen and Miller, 1991, sheet 1) and in the agricultural area near Casa Grande (Thomsen and Baldys, 1985, sheet 1), the direction of ground-water movement has been altered and is toward the pumping centers. Ground-water discharge from aquifers in these areas occurs by evapotranspiration, discharge to streams as base flow, underflow to downgradient basins, and by groundwater pumping in the agricultural and urban areas (Anderson and others, 1992).

\section{Population and Land Use}

The two largest population centers in the Basin and Range Lowlands of the CAZB are the metropolitan areas of Phoenix and Tucson (fig. 3). Of the 3,665,230 people counted in 1990 in Arizona by the U.S. Census Bureau, 53 percent resided in the metropolitan area of Phoenix and 16 percent resided in the metropolitan area of Tucson (U.S. Bureau of the Census, 1991). Population growth in the State of Arizona between 1990 and 2000 was 40 percent (Arizona Department of Economic Security, rev. March 27, 2001).

Land use in the Basin and Range Lowlands, excluding land in Mexico, primarily is rangeland (74 percent; fig. 3). Most of the remaining area is forest (7 percent), urban ( 8 percent), or agricultural (8 percent). In 1990, cotton comprised 68 percent of the crop acreage in the CAZB; alfalfa and other hay comprised 13 percent, and wheat and barley comprised 11 percent (Arizona Agricultural Statistics Service, 1991). The compilation of the digital land-use and land-cover geographic information system data for the CAZB study area (fig. 3) is described in more detail in Cordy and others (1998).

The Sierra Vista subbasin was considered to have minimal urban development (Gellenbeck and Coes, 1999). The population in the area of Sierra Vista is one percent of the total in Arizona (U.S. Bureau of the
Census, 1991). This subbasin has a small amount of urban ( 2 percent) and agricultural (1 percent) lands. Most of the land area is rangeland ( 83 percent) or forest (13 percent).

In the West Salt River Valley and the Upper Santa Cruz Basin, the land use has more urban development than in the Sierra Vista subbasin. As of 1990, 40 percent of the land in the West Salt River Valley was rangeland, 32 percent was urban, and 25 percent was agricultural. As of 1990, 60 percent of the land in the Upper Santa Cruz Basin was rangeland, 14 percent was forest, 22 percent was urban, and 2 percent was agricultural. The metropolitan areas of Phoenix and Tucson in the West Salt River Valley and the Upper Santa Cruz Basin, respectively, have had extensive population growth over the last decade; consequently, the urban land-use area more than doubled in the CAZB from 1980 to 1990 (Cordy and others, 1998). These population and land-use changes could have possible effects on water use, quantity, and quality (Cordy and others, 1998).

\section{Sources of Organic Compounds}

Information about sources of pesticides and VOCs in the CAZB is difficult to obtain and summarize because those types of data were not collected in the past. The available data for pesticides are not complete owing to the lack of historical data compilation; therefore, estimates of applications and releases used in this report are probably lower than the amounts actually used. Information about VOCs released to the environment is even less complete, and most of this information is based on known contamination sites. Because sources of VOCs are varied, the information about VOC releases is general.

\section{Pesticides}

In Arizona, the amount of pesticides used in agricultural areas has been estimated using various methods. Sales data derived from voluntary questionnaires provide some information about pesticide use in Arizona. Limitations of these data include: (1) the data cannot be correlated with usage because the compounds may have been used outside Arizona, or in a different year than when they were purchased, and (2) the data are based on voluntary surveys completed by distributors (Brew and Baker, 1987). 


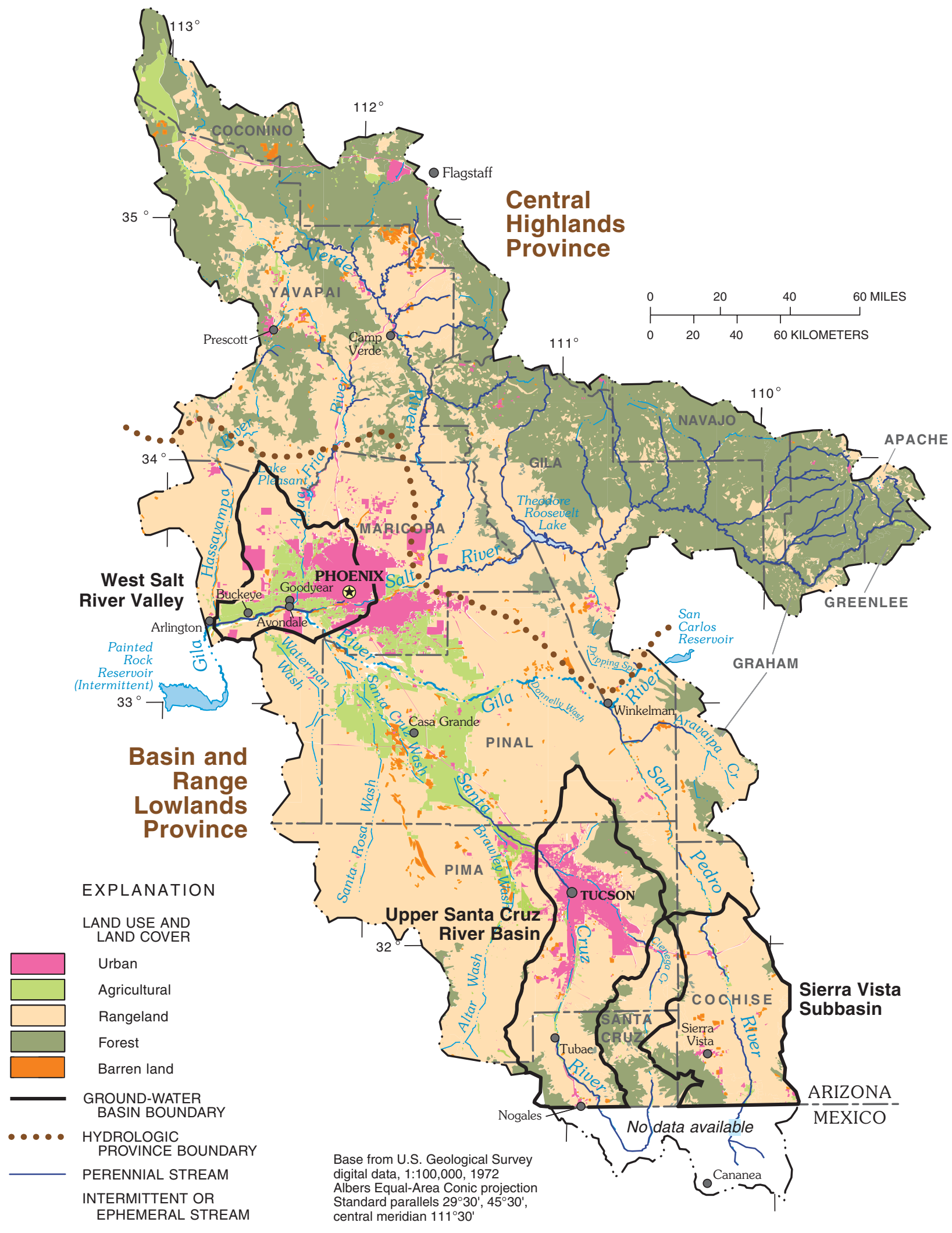

Figure 3. Land use and land cover in the Central Arizona Basins study area. 
The small number of respondents to these surveys distorts the resulting data. Because of a lack of participation and regulations that require information about pesticide application, the sales survey is being replaced by a computer database (Ken Agnew, Pesticide Information and Training Office, University of Arizona, oral commun., 1999). Data on pesticide use in this database will be more accurate and complete than the sales data and will include acres treated, pounds of active ingredient, and date of application. Because reporting is mandated only for commercial applications, the information on pesticide use in Arizona will not be complete; however, the data will be more extensive than the historical data.

Accurate information on the amounts of pesticides used in Arizona is not available for historical periods because of the unreliable nature of the pesticide-use information from the sales data. The best description of pesticide use in Arizona through time may be the trends in the use of specific pesticides on cotton compiled from questionnaires and personal interviews with entomologists, extension agents, pest-control advisors, applicators, and growers (Brew and Baker, 1987). Because cotton has been a major crop in Arizona and more insecticides are applied to cotton than other crops (Brew and Baker, 1987), the summary of this information can provide some insight into the pesticides used in Arizona. One important trait from these historical data is the use of the same compounds over several decades, including the insecticides sulfur and parathion and the herbicides sodium chlorate and trifluralin (table 1).

Dichlorodiphenyldichloroethane (DDT) and toxaphene were used in the late 1940s, 1950s, and early 1960s on cotton crops in Arizona. DDT was used to eradicate the lygus bug, cotton leaf perforater, and the pink bollworm from cotton crops (Brew and Baker, 1987). In 1965, because of contamination of milk and hay, Arizona became the first state in the United States to restrict the use of DDT, and in 1969, a total restriction for use was enacted (Brew and Baker, 1987). Toxaphene also was used to eradicate pests on cotton and other crops in Arizona (Brew and Baker, 1987). Milk contamination and the reduced effectiveness of toxaphene resulted in its removal from the recommended list of pesticides in Arizona in 1976 (Brew and Baker, 1987).

Pesticide use on crops in Arizona changed dramatically in the 1970s. The most notable changes were the increased use of herbicides and decreased amount of insecticides used on crops because of the development of synthetic pyrethroid insecticides that could be used in smaller amounts (Brew and Baker, 1987). The development of biological pest control also reduced the amount of pesticides applied.

The trend toward smaller amounts of pesticides applied to crops continued in the 1980s because several Federal and State regulations limited the use of pesticides. In addition, limits on ground-water withdrawals resulted in decreased irrigation and a reduction in the area under cultivation. The increased use of pheromones and other biological techniques to reduce the effects of pests also helped to reduce the need for pesticides.

Data for general pesticide use in Arizona in the 1990s are not available; therefore, pesticide use in Arizona was characterized on the basis of a summary of the pesticides used on cotton crops in the United States (Padgitt, 1997). In the early 1990s, the use of herbicides on cotton remained similar to use in the 1980s. The use of insecticides on cotton crops increased in the mid-1990s because of expanded acreage and more intensive treatments per acre. The amounts used in the 1990s may be larger than those used in the 1980s; however, the amounts are less than half the estimated amounts used in the 1960s and 1970s (Padgitt, 1997).

The amount of pesticides applied in agricultural areas where ground-water and (or) surface-water samples were collected in 1996-98 for analyses of organic compounds varies greatly on the basis of location (table 2). Estimates are not available for pesticide use in other land-use types. The highest pesticide usage during the sampling period was in the West Salt River Valley, which historically had some of the largest areas of agriculture in the State (Brew and Baker, 1987). In a study of shallow ground water in the western part of the West Salt River Valley, a small area of primarily agricultural land use accounted for about one-half of the pesticides used in this basin (table 2). Some pesticides were used in the Upper Santa Cruz Basin, but in general, the amounts are insignificant compared to the amounts of pesticides used in the West Salt River Valley. Only three pesticides were reportedly used in the Sierra Vista subbasin during 1996-98 (table 2). 
Table 1. Recommended insecticides, herbicides, and defoliants for cotton crops in Arizona, 1940-87

[Lists are in order of preference of use; Source: Brew and Baker (1987)]

\begin{tabular}{|c|c|c|c|c|c|c|}
\hline $1940-45$ & 1955 & 1965 & 1970 & 1975 & 1980 & $1985-87$ \\
\hline \multicolumn{7}{|c|}{ Insecticides } \\
\hline $\begin{array}{l}\text { Sulfur } \\
\text { Cryolite } \\
\text { Sodium arsenite } \\
\text { Calcium arsenate } \\
\text { Nicotene sulfate } \\
\text { Pyrethrum } \\
\text { Sabadilla } \\
\text { Paris green } \\
\text { Rotenone } \\
\text { Calcium cyanide } \\
\text { Carbon bisulfide }\end{array}$ & $\begin{array}{l}\text { Toxaphene } \\
\text { DDT } \\
\text { Sulfur } \\
\text { Parathion } \\
\text { BHC } \\
\text { Demeton } \\
\text { Aramite } \\
\text { Dieldrin } \\
\text { Endothall }\end{array}$ & $\begin{array}{l}\text { DDT } \\
\text { Toxaphene } \\
\text { Endosulfan } \\
\text { Malathion } \\
\text { Dicrotophos } \\
\text { Azinphos-methyl } \\
\text { Methyl parathion } \\
\text { Parathion } \\
\text { Dimethoate } \\
\text { Sulfur } \\
\text { Dibrom }\end{array}$ & $\begin{array}{l}\text { Azinphos-methyl } \\
\text { Monocrotophos } \\
\text { Toxaphene } \\
\text { Methyl parathion } \\
\text { Parathion } \\
\text { Dimethoate } \\
\text { Malathion } \\
\text { Sulfur } \\
\text { Dicofol }\end{array}$ & $\begin{array}{l}\text { Methyl parathion } \\
\text { Azinphos-methyl } \\
\text { Toxaphene } \\
\text { Chlordimeform } \\
\text { Methomyl } \\
\text { Monocrotophos } \\
\text { Aldicarb } \\
\text { Dicofol } \\
\text { Dimethoate } \\
\text { Carbaryl } \\
\text { Dicrotophos }\end{array}$ & $\begin{array}{l}\text { Chlordimeform } \\
\text { Permethrin } \\
\text { Azinphos-methyl } \\
\text { Fenvalerate } \\
\text { Methyl parathion } \\
\text { Methomyl } \\
\text { Monocrotophos } \\
\text { Sulprofos } \\
\text { Gossyplure } \\
\text { EPN } \\
\text { Dimethoate } \\
\text { Aldicarb } \\
\text { Chlorpyrifos } \\
\text { Dicofol } \\
\text { Propargite } \\
\text { Sulfur } \\
\text { Dicrotophos } \\
\text { Methidathion }\end{array}$ & $\begin{array}{l}\text { Chlordimeform } \\
\text { Methyl parathion } \\
\text { Cypermethrin } \\
\text { Fenvalerate } \\
\text { Methomyl } \\
\text { Monocrotophos } \\
\text { Azinphos-methyl } \\
\text { Gossyplure } \\
\text { Acephate } \\
\text { Permethrin } \\
\text { Malathion } \\
\text { Propargite } \\
\text { Oxamyl } \\
\text { Dicofol } \\
\text { Dimethoate } \\
\text { Chlorpyrifos } \\
\text { Aldicarb } \\
\text { Sulfur } \\
\text { Sulprofos } \\
\text { Flucythrinate }\end{array}$ \\
\hline \multicolumn{7}{|c|}{ Herbicides/Defoliants } \\
\hline Sodium chlorate & $\begin{array}{l}\text { Sodium chlorate } \\
\text { Diuron } \\
\text { Dalapon } \\
\text { Minuron } \\
\text { TCA }\end{array}$ & $\begin{array}{l}\text { Sodium chlorate } \\
\text { Diuron } \\
\text { Trifluralin } \\
\text { Dalapon } \\
\text { TCA } \\
\text { Monuron }\end{array}$ & $\begin{array}{l}\text { Sodium chlorate } \\
\text { Trifluralin } \\
\text { Dalapon } \\
\text { Diuron } \\
\text { Monuron } \\
\text { Chlorothal }\end{array}$ & $\begin{array}{l}\text { Sodium chlorate } \\
\text { Trifluralin } \\
\text { Prometryn } \\
\text { MSMA } \\
\text { Diuron } \\
\text { Profluralin } \\
\text { Chlorothal } \\
\text { Bensulide }\end{array}$ & $\begin{array}{l}\text { Sodium chlorate } \\
\text { Trifluralin } \\
\text { Prometryn } \\
\text { MSMA } \\
\text { DSMA } \\
\text { Pendimethalin } \\
\text { Glyphosate } \\
\text { Diuron } \\
\text { Profluralin }\end{array}$ & $\begin{array}{l}\text { DEF } \\
\text { Sodium chlorate } \\
\text { Trifluralin } \\
\text { Prometryn } \\
\text { Diuron } \\
\text { Glyphosate } \\
\text { Fluazifop-butyl } \\
\text { Arsenic acid } \\
\text { MSMA } \\
\text { Pendimethalin } \\
\text { Paraquat } \\
\text { Sethoxydin } \\
\text { Cyanazine }\end{array}$ \\
\hline
\end{tabular}

\section{Volatile Organic Compounds}

Information about sources of VOCs is not as readily available as information about pesticides; therefore, there is a general lack of information about the origin of VOCs in the CAZB. One source of information is the Toxics Release Inventory (TRI) maintained by the USEPA (U.S. Environmental Protection Agency, 1998). Data for 1995 indicate that no compounds were released to water in the CAZB, but several compounds were released to air (Arizona Department of Environmental Quality, 1997).
Transport of VOCs from air to water is possible (Squillace and others, 1999). Some of the compounds released to air in the CAZB in 1995 include 1,1,1-trichloroethane, trichloroethene (TCE), and trichloromethane (Arizona Department of Environmental Quality, 1997). Information from the TRI data indicates that most of the VOC release sites are in the metropolitan areas of Phoenix and Tucson (U.S. Environmental Protection Agency, 1998). Because of possible transport of VOCs from the atmosphere to the ground water, detection of VOCs in the ground water near the release sites is possible. 
Table 2. Pesticides applied in the ground-water study areas, Central Arizona Basins study area, 1996-98

[Source: Ken Agnew, Pesticide Information and Training Office, University of Arizona, written commun. (1999). Values are in pounds of active ingredient. Only pesticides analyzed in ground-water and surface-water samples are included]

Ground-water study areas

\begin{tabular}{|c|c|c|c|c|c|c|c|c|c|c|c|c|}
\hline \multirow{3}{*}{$\begin{array}{l}\text { Compound } \\
\text { Name }\end{array}$} & \multicolumn{6}{|c|}{ West Salt River Valley } & \multirow{2}{*}{\multicolumn{3}{|c|}{ Upper Santa Cruz Basin }} & \multirow{2}{*}{\multicolumn{3}{|c|}{ Sierra Vista Subbasin }} \\
\hline & \multicolumn{3}{|c|}{$\begin{array}{l}\text { Agricultural land-use } \\
\text { study area }\end{array}$} & \multicolumn{3}{|c|}{ Basinwide } & & & & & & \\
\hline & 1996 & 1997 & 1998 & 1996 & 1997 & 1998 & 1996 & 1997 & 1998 & 1996 & 1997 & 1998 \\
\hline $2,4-\mathrm{D}$ & 514 & 274 & 499 & 3,584 & 569 & 994 & 0 & 0 & 0 & 0 & 0 & 0 \\
\hline Aldicarb & 2,392 & 1,321 & 584 & 7,834 & 2,782 & 2,319 & 3,011 & 4,549 & 0 & 0 & 0 & 0 \\
\hline Atrazine & 1,367 & 851 & 136 & 1,814 & 1,052 & 749 & 0 & 0 & 0 & 0 & 0 & 0 \\
\hline Azinphos-methyl & 0 & 30 & 0 & 0 & 30 & 0 & 0 & 0 & 0 & 0 & 0 & 0 \\
\hline Bromoxynil & 0 & 0 & 21 & 45 & 0 & 141 & 85 & 93 & 70 & 0 & 0 & 0 \\
\hline Carbaryl & 0 & 773 & 0 & 881 & 1,475 & 795 & 0 & 0 & 0 & 0 & 0 & 0 \\
\hline Carbofuran & 440 & 273 & 277 & 1,198 & 474 & 685 & 0 & 0 & 0 & 0 & 0 & 0 \\
\hline Chlorothalonil & 0 & 0 & 0 & 257 & 140 & 1,110 & 0 & 0 & 0 & 0 & 0 & 0 \\
\hline Chlorpyrifos & 14,472 & 6,953 & 1,989 & 30,061 & 13,956 & 5,448 & 367 & 0 & 396 & 0 & 70 & 0 \\
\hline Cyanazine & 0 & 939 & 0 & 1,037 & 2,187 & 0 & 0 & 0 & 0 & 0 & 0 & 0 \\
\hline DCPA & 0 & 0 & 0 & 144 & 168 & 168 & 0 & 0 & 0 & 0 & 0 & 0 \\
\hline Diazinon & 0 & 0 & 0 & 503 & 163 & 147 & 0 & 0 & 0 & 0 & 0 & $c$ \\
\hline Dicamba & 77 & 21 & 161 & 851 & 264 & 898 & 0 & 0 & 0 & 0 & 0 & 0 \\
\hline Dichloropropene & 130,227 & 63,240 & 98,175 & 178,332 & 112,992 & 126,963 & 0 & 0 & 0 & 0 & 0 & 0 \\
\hline Dimethoate & 329 & 834 & 568 & 3,056 & 3,752 & 2,462 & 0 & 40 & 0 & 0 & 0 & c \\
\hline Disulfoton & 922 & 868 & 862 & 1,474 & 1,216 & 1,574 & 0 & 0 & 0 & 0 & 0 & 0 \\
\hline Diuron & 147 & 105 & 77 & 374 & 423 & 279 & 112 & 2 & 0 & 0 & 1 & 0 \\
\hline EPTC & 1,412 & 902 & 752 & 8,529 & 9,783 & 9,950 & 0 & 0 & 0 & 0 & 0 & c \\
\hline Esfenvalerate & 3 & 0 & 0 & 364 & 124 & 118 & 0 & 0 & 0 & 0 & 0 & 0 \\
\hline Malathion & 1,018 & 5,584 & 3,764 & 4,711 & 12,427 & 7,618 & 2,493 & 1,596 & 3,754 & 0 & 0 & 0 \\
\hline MCPA & 156 & 56 & 83 & 1,398 & 576 & 1,988 & 0 & 0 & 0 & 0 & 0 & 0 \\
\hline Methomyl & 105 & 1,558 & 137 & 2,673 & 3,238 & 2,447 & 0 & 60 & 185 & 22 & 47 & 23 \\
\hline Methyl parathion & 6,285 & 2,489 & 914 & 7,827 & 4,858 & 1,394 & 89 & 165 & 218 & 0 & 0 & 0 \\
\hline Metolachlor & 0 & 0 & 0 & 40 & 0 & 0 & 0 & 0 & 0 & 0 & 0 & 0 \\
\hline Metribuzin & 0 & 0 & 0 & 220 & 93 & 890 & 0 & 0 & 0 & 0 & 0 & 0 \\
\hline Norflurazon & 0 & 0 & 0 & 16 & 0 & 0 & 0 & 0 & 0 & 0 & 0 & 0 \\
\hline Oryzalin & 0 & 0 & 0 & 1,834 & 2,860 & 1,530 & 0 & 0 & 0 & 0 & 0 & 0 \\
\hline Oxamyl & 3,217 & 2,776 & 657 & 6,567 & 3,800 & 935 & 347 & 128 & 0 & 0 & 0 & 0 \\
\hline Pendimethalin & 2,928 & 2,056 & 1,991 & 3,426 & 2,887 & 2,656 & 45 & 0 & 0 & 0 & 0 & 0 \\
\hline Permethrin & 43 & 215 & 339 & 679 & 751 & 1,064 & 0 & 0 & 0 & 0 & 0 & 0 \\
\hline Phorate & 436 & 268 & 305 & 436 & 268 & 305 & 0 & 0 & 0 & 0 & 0 & 0 \\
\hline Trifluralin & 3,327 & 4,276 & 10,061 & 15,421 & 12,282 & 16,249 & 0 & 13 & 0 & 16 & 0 & 0 \\
\hline Totals & 169,817 & 96,662 & 122,352 & 285,586 & 195,590 & 191,876 & 6,549 & 6,646 & 4,623 & 38 & 118 & 23 \\
\hline
\end{tabular}


The ADEQ has identified VOCs, which include gasoline compounds, solvents, and refrigerants, as contaminants of high concern in ground water in Arizona (Marsh, 1994). Leaking underground-storage tanks and disposal of solvents were linked to most of the documented cases of ground water contaminated by VOCs. High-technology manufacturing facilities (mostly electronics and aerospace industries) use solvents for degreasing and are in the urban areas of the State where most of the ground water that is contaminated by VOCs has been identified. Disposal of solvents has occurred since the 1950s and probably earlier. Recent contamination of ground water by VOCs is attributed to disposal or leaks at dry-cleaning facilities. Use of some public drinking-water wells in Phoenix and Tucson has been discontinued because of contamination by VOCs (Marsh, 1994).

\section{STUDY DESIGN}

The study design for the CAZB was based on NAWQA guidelines and on the major water-quality issues in the study area. The major water-quality issues in the CAZB study area include: effects of discharge of treated-sewage effluent on surface-water and groundwater quality, aquatic life, and instream flows near urban areas; movement and fate of organic contaminants in ground water from industrial discharges, spills, landfills, and other point sources in urban areas; and movement and fate of fertilizers, pesticides, and other contaminants from nonpoint sources such as irrigation-return flow and stormwater runoff (Cordy, 1994). These issues were the primary basis for selecting sites for sampling of organic compounds in ground water and surface water.

The study design used for this project is an attempt to characterize the occurrence and distribution of water quality in the ground-water and surface-water resources that are used in the CAZB. The results presented in this report accurately represent the water quality at the specific sites sampled and only generally characterize the entire resource.

\section{Ground Water}

The NAWQA ground-water study is designed to assess water-quality conditions of the major aquifers in each study area (Gilliom and others, 1995). Sampling sites used during 1996-98 within the CAZB study area included two basins where ground water is used in large quantities and ground-water quality has been, or has the potential to be, affected by human activities and one basin where ground-water quality was not expected to be affected by human activities. The basins chosen for study include the West Salt River Valley, Upper Santa Cruz Basin, and Sierra Vista subbasin (fig. 4). Two different types of NAWQA ground-water studies were completed in the CAZB in the chosen sampling areas-subunit surveys (SUSs) and land-use studies (LUSs).

\section{Sub-Unit Survey}

Three SUSs were conducted in the CAZB in 1996-98. The purpose of NAWQA SUSs is to broadly characterize the water quality of major aquifer systems in a study area primarily by sampling existing wells (Gilliom and others, 1995). Areas where ground water is being used for industry, irrigation, or public or domestic water supply are given priority for sampling over those areas where ground water is not used. Of water being used in the CAZB, 96 percent is used in the Basin and Range Lowlands. Of this amount, 80 percent is drawn from ground-water resources (Cordy and others, 1998). The largest use of ground water is for agriculture followed by municipal supply (Cordy and others, 1998). Increased amounts of goodquality drinking water will be needed in those areas where the population is growing fastest, specifically in the West Salt River Valley and the Upper Santa Cruz Basin. Consequently, these two areas were prioritized for study. The likelihood of degradation of groundwater quality, such as that from high nitrate concentrations and the existence of pesticides and VOCs, also was a factor in the prioritizing of these two basins. An SUS was completed in the Sierra Vista subbasin to provide a comparison with conditions in the West Salt River Valley and the Upper Santa Cruz Basin because the Sierra Vista subbasin was thought to be minimally affected by human activities (Coes and others, 1999). 


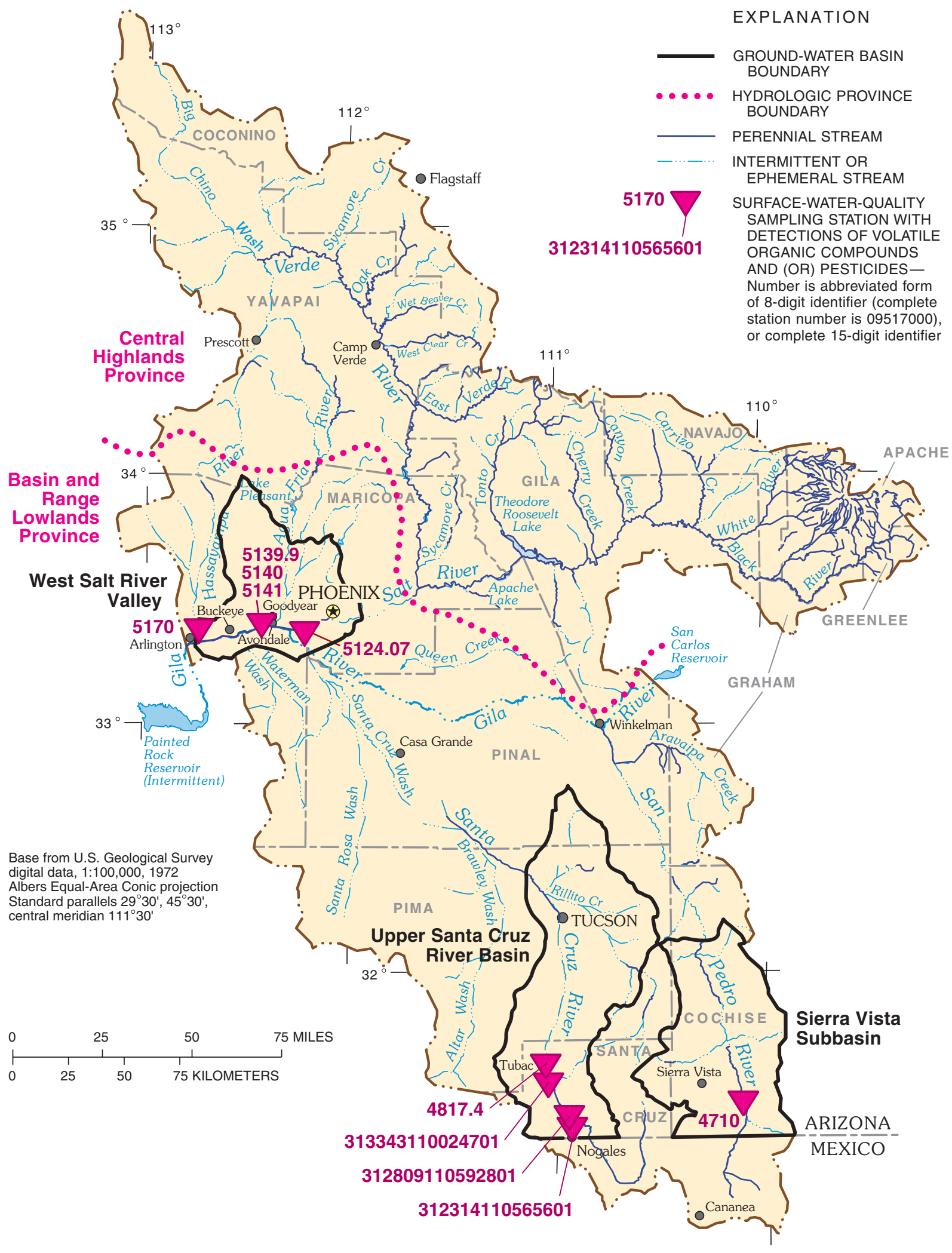

Figure 4. Surface-water sites and ground-water basins where samples were collected for pesticide and volatile organic compound analyses, Central Arizona Basins study area, 1996-98. 
The following statistically based, stratifiedrandom approach was used to select existing wells for each SUS. Computer software (Scott, 1990) was used to divide each SUS into a specific number of equal-area polygons, referred to as cells. Within each cell, a suitable existing well was found for sampling near a point chosen randomly by the computer software. A well was suitable for sampling if it had a submersible pump, a sampling point between the pump and any treatment system and (or) storage tanks, a measuring point to determine depth to water; if construction information (depth, perforated interval, casing diameter, and driller's log) was available; and if the well owner gave permission to sample. For some locations, a well with all of the characteristics listed above was not available; in these cases, wells were sampled that did not have all of the characteristics.

Sixty-four existing wells in the West Salt River Valley were sampled during 1996-97 (table 3). Water from these wells is used for domestic and municipal drinking water, irrigation, livestock, and (or) industrial uses. The computer software (Scott, 1990) was used to select 35 wells from the 64 sampled as the basis for characterizing the ground-water quality in the West Salt River Valley and for comparisons with results from other ground-water studies in the CAZB. All of these wells were sampled for pesticide analyses, and 30 were sampled for VOC analyses. Depth of the 35 wells ranged from 100 to $1,445 \mathrm{ft}$ below land surface.

Twenty-nine wells were sampled in the Upper Santa Cruz Basin SUS in 1998 (table 3). Depth of the wells ranged from 40 to $800 \mathrm{ft}$ below land surface. Water from these wells is used for domestic drinking water, irrigation, livestock, and (or) industrial uses. Thirty equal-area cells were used for well selection. A suitable well for sampling could not be found in one cell.

Nineteen wells were sampled in the Sierra Vista subbasin SUS in 1996 (table 3). Depth of the wells ranged from 90 to $1,003 \mathrm{ft}$ below land surface. Water from these wells is used for domestic drinking water, irrigation, livestock, and (or) industrial uses. Twenty equal-area cells were used for this SUS. A suitable well for sampling could not be found in one cell. Groundwater samples were analyzed for pesticides using capillary-column gas chromatography/mass spectrometry with selected-ion monitoring (GCMS). Samples were not collected for high-performance liquid chromatography (HPLC) analyses because of the low percentage of agricultural land use in the basin (see section entitled "Field and Laboratory Methods").

Table 3. Study areas where ground-water samples were collected for pesticide and (or) volatile organic compound analyses, Central Arizona Basins study area, 1996-98

[HPLC, high-performance liquid chromatography; GCMS, gas chromatography/mass spectrometry; PT/GCMS, purge and trap gas chromatography/mass spectrometry]

Number of samples

\begin{tabular}{|c|c|c|c|c|}
\hline \multirow[b]{3}{*}{ Study area } & \multirow[b]{3}{*}{ Sampling period } & & & \\
\hline & & \multicolumn{2}{|c|}{ Pesticides } & \multirow{2}{*}{$\begin{array}{l}\text { Volatile organic compounds } \\
\text { PT/GCMS }\end{array}$} \\
\hline & & HPLC & GCMS & \\
\hline \multicolumn{5}{|l|}{ West Salt River Valley } \\
\hline Sub-unit survey ${ }^{1}$ & 02-96 to $09-97$ & 64 & 64 & 52 \\
\hline Agricultural land-use study ${ }^{2}$ & 08-97 and 02-98 & 18 & 18 & 18 \\
\hline Upper Santa Cruz Basin sub-unit survey & 04-98 to $07-98$ & 29 & 29 & 29 \\
\hline Sierra Vista subbasin sub-unit survey & $06-96$ to $10-96$ & 0 & 19 & 19 \\
\hline
\end{tabular}

${ }^{1}$ Only 35 wells are used in this report.

${ }^{2}$ Nine wells were sampled one time in August 1997 and one time in February 1998. 


\section{Agricultural Land-Use Study}

One LUS was completed in the CAZB in 1997-98. The purpose of NAWQA LUSs is to assess the concentrations and distribution of water-quality constituents in shallow ground water recharged in the last 10 years beneath the most significant land-use settings (Gilliom and others, 1995). The CAZB LUS characterizes the effects of agricultural activities in the southwestern part of the West Salt River Valley. This study included drilling, constructing, and sampling nine monitoring wells that range in depth from 20 to $83 \mathrm{ft}$ below the land surface and have 5-foot perforated intervals within the top $10 \mathrm{ft}$ of the water table. Drilling sites were located by identifying an area in the West Salt River Valley that had primarily agricultural land use and depths to ground water that were less than $100 \mathrm{ft}$. Ten equal-area cells were used for site selection; nine sites were acceptable for installation of wells. Construction data for these wells are available in Tadayon and others (1999); all wells have polyvinylchloride casing and screens. Sampling of these wells occurred at two different times during the growing season-August 1997 at the end of the intensive irrigation season (summer months) and February 1998 before the beginning of the intensive irrigation season (table 3).

\section{Surface Water}

The NAWQA surface-water studies focus on assessing the most important water-quality conditions of streams and rivers in each study unit (Gilliom and others, 1995). In the CAZB study area, the most important surface-water quality concerns are the effects of discharge of treated-sewage effluent, irrigationreturn flow, and stormwater runoff (Cordy, 1994). Eleven surface-water sites were used in the sampling network in the CAZB. Samples for analyses of pesticides and VOCs were collected from four sites (fig. 4; table 4). In addition to surface-water sites sampled by CAZB personnel, three sites were sampled on the Santa Cruz River, and one site was sampled on Nogales Wash as part of another project (fig. 4; table 4). Sampling and analytical procedures were the same as those used at CAZB sites. Data from these sites are discussed in this report. Surface-water samples were collected during two streamflow conditions: lowflow samples, collected during normal streamflow conditions, and high-flow samples, collected during storm runoff.
Two types of surface-water sampling sites are included in the NAWQA design-basic fixed sites and intensive fixed sites. Basic fixed sites were to be used to "characterize the spatial and temporal distribution of general water quality and constituent transport in relation to hydrologic conditions and contaminant sources" (Gilliom and others, 1995). Intensive fixed sites were to be used to "characterize seasonal and short-term temporal variability of general water-quality and constituent transport and determine the occurrence and seasonal patterns in concentrations and transport of dissolved pesticides" (Gilliom and others, 1995).

The main difference between the use of basic fixed sites and the use of intensive fixed sites is the frequency of sampling. High frequency sampling at a few intensive fixed sites during key time periods can provide more useful information compared with monthly sampling at the basic fixed sites. Additionally, the surface-water sites are classified either as integrators (sites that are used to characterize the water quality for large, heterogeneous basins that are affected by a combination of possible point, nonpoint, and natural contaminant sources) or indicators (sites that are used to characterize the water quality for smaller, homogeneous basins that are affected by specific point, nonpoint, or natural contaminant sources). Additional samples for pesticide and VOC analyses were collected at four sites along the Santa Cruz River near Tubac during 1997-98 as part of a separate USGS study.

\section{Basic Fixed Sites}

Samples for pesticide and VOC analyses were collected at two basic fixed sites (table 4)-San Pedro River at Charleston (09471000) and 91st Avenue wastewater-treatment plant outfall near Phoenix (09512407).

The site on the San Pedro River in the Sierra Vista subbasin was selected as a reference site for the Basin and Range Lowlands. The San Pedro River is one of the last free-flowing rivers in this hydrologic province (Cordy and others, 1998). VOCs were not analyzed in water from this site because typical sources of VOCs including urban and industrial land-use activities are not widespread in the drainage area. To determine the effect of agricultural activities adjacent to the river in the basin, six samples collected at this site from January to October 1997 were analyzed for pesticides (table 4). 
Table 4. Surface-water sites where samples were collected for pesticide and (or) volatile organic compound analyses, Central Arizona Basins study area, 1996-98

[HPLC, high-performance liquid chromatography; GCMS, gas chromatography/mass spectrometry; PT/GCMS, purge and trap gas chromatography/mass spectrometry; WSRV, West Salt River Valley; USC, Upper Santa Cruz Basin; BFS, basic fixed site; IFS, intensive fixed site; IND, indicator site; INT, integrator site]

\begin{tabular}{|c|c|c|c|c|c|c|c|}
\hline \multirow[b]{3}{*}{ Site name } & \multirow[b]{3}{*}{ Site number } & \multirow{3}{*}{$\begin{array}{c}\text { Ground- } \\
\text { water basin }\end{array}$} & \multirow[b]{3}{*}{ Type of site } & \multirow[b]{3}{*}{ Sampling period } & \multicolumn{3}{|c|}{ Number of samples } \\
\hline & & & & & \multicolumn{2}{|c|}{ Pesticides } & \multirow{2}{*}{$\begin{array}{c}\text { Volatile organic } \\
\text { compounds } \\
\text { PT/GCMS }\end{array}$} \\
\hline & & & & & HPLC & GCMS & \\
\hline \multicolumn{8}{|c|}{ Sites used in this study } \\
\hline San Pedro River at Charleston & 09471000 & $\begin{array}{l}\text { Sierra Vista } \\
\text { subbasin }\end{array}$ & BFS-IND & $01-97$ to $10-97$ & 6 & 6 & 0 \\
\hline $\begin{array}{l}\text { 91st Avenue waste-water treatment plant } \\
\text { outfall near Phoenix }\end{array}$ & 09512407 & WSRV & BFS-IND & $06-96$ to $04-97$ & 0 & 0 & 6 \\
\hline Gila River at Buckeye Canal: & & WSRV & IFS-INT & & & & \\
\hline $\begin{array}{l}\text { Gila River above diversions, at the head of } \\
\text { Buckeye Canal }{ }^{1}\end{array}$ & 09513990 & & & $02-96$ to $03-96$ & 2 & 2 & 0 \\
\hline Buckeye Canal near Avondale & 09514000 & & & $\begin{array}{l}12-96 \text { to } 02-98 \\
06-96 \text { to } 04-97\end{array}$ & $\begin{array}{r}28 \\
0\end{array}$ & $\begin{array}{r}28 \\
0\end{array}$ & $\begin{array}{l}0 \\
5\end{array}$ \\
\hline $\begin{array}{l}\text { Gila River at Estrella Parkway near } \\
\text { Goodyear }^{2}\end{array}$ & 09514100 & & & 04-98 & 1 & 1 & 0 \\
\hline Hassayampa River near Arlington & 09517000 & WSRV & IFS-IND & $02-96$ to $02-98$ & 40 & 40 & 0 \\
\hline & & & & $06-96$ to $12-96$ & 0 & 0 & 2 \\
\hline \multicolumn{8}{|c|}{ Sites used in separate study } \\
\hline $\begin{array}{l}\text { Nogales Wash near the Nogales } \\
\text { International Wastewater Treatment Plant }\end{array}$ & 312314110565601 & USC & --- & $\begin{array}{l}11-97 \\
06-98\end{array}$ & $\begin{array}{l}1 \\
1\end{array}$ & $\begin{array}{l}1 \\
1\end{array}$ & $\begin{array}{l}1 \\
1\end{array}$ \\
\hline Santa Cruz River near Rio Rico, Arizona & 312809110592801 & USC & --- & $\begin{array}{l}11-97 \\
06-98\end{array}$ & $\begin{array}{l}1 \\
1\end{array}$ & $\begin{array}{l}1 \\
1\end{array}$ & $\begin{array}{l}1 \\
1\end{array}$ \\
\hline Santa Cruz River at Santa Gertrudis Lane & 313343110024701 & USC & --- & $\begin{array}{l}11-97 \\
06-98\end{array}$ & $\begin{array}{l}1 \\
1\end{array}$ & $\begin{array}{l}1 \\
1\end{array}$ & $\begin{array}{l}1 \\
1\end{array}$ \\
\hline Santa Cruz River at Tubac, Arizona & 09481740 & USC & --- & $\begin{array}{l}11-97 \\
06-98\end{array}$ & $\begin{array}{l}1 \\
1\end{array}$ & $\begin{array}{l}1 \\
1\end{array}$ & $\begin{array}{l}1 \\
1\end{array}$ \\
\hline
\end{tabular}

\footnotetext{
${ }^{1}$ Site used before site 09514000 was established.

2 Site used when high flows were present at 09514000.
}

The site at the 91st Avenue WWTP is within the West Salt River Valley and was selected as an indicator of treated-sewage effluent. Water-quality data from this basic fixed site were used to characterize the effect of sewage effluent at an intensive fixed site that is downstream, Gila River at Buckeye Canal. Because the input to the site at the WWTP includes water collected from a large urban area, six samples collected from June 1996 to April 1997 were analyzed for VOCs. Only a small number of samples were collected because high temperatures during most of the year can cause volatilization of most VOCs from the surface water.

\section{Intensive Fixed Sites}

Samples for pesticide and VOC analyses were collected at two intensive fixed sites (table 4) - the Hassayampa River near Arlington (09517000) and the Gila River at Buckeye Canal. On the basis of flow conditions, three different sampling locations were used during 1996-98 for the site on the Gila River at Buckeye Canal. During periods of low flow, all of the flow in the river is diverted into Buckeye Canal, whereas, during high flows only a portion of the low is diverted to protect the canal. Initially, NAWQA samples were collected during low flow at the Gila 
River above diversions at the head of Buckeye Canal (09513990). In May 1996, the sampling site was moved $0.3 \mathrm{mi}$ downstream in the canal (Buckeye Canal near Avondale- 09514000) to take advantage of better sampling conditions. During a single high flow in April 1998, a sample was collected at the Gila River at Estrella Parkway near Goodyear (09514100) because most of the flow was in the river channel.

Despite their varied locations, samples from these sites were considered representative of flow at the Gila River at Buckeye Canal, and they are treated in this report as a single set of samples from the site.

The site, Gila River at Buckeye Canal, was selected as an integrator site for characterizing streamflow leaving the CAZB study area. Because surface-water diversions in the CAZB study area and specifically within the Phoenix metropolitan area (fig. 2) are complex, the sources of surface water at this site are not always the same. Water quality is representative of different hydrologic provinces of the CAZB study area depending on the time of sample collection. For most samples, the source of surface water was base flow from the Gila River that is generally discharge from the 91st Avenue WWTP and ground-water inflow. Some samples included flow from rivers in the Central Highlands Province owing to releases from reservoirs upstream. Samples were collected for pesticide and VOC analyses at this site to determine the effects of local agricultural activities and upstream urban land use.

The Hassayampa River site was selected as an indicator site of agricultural activities in the CAZB. Two major irrigation canals-Buckeye Canal and Roosevelt Canal-drain into the river channel above the sampling site. The Hassayampa River is ephemeral above these canal inflow points. The source of water at this site is irrigation-return flow from these canals except during periods of runoff. Samples were collected at this site and analyzed for pesticides to determine the effects of the agricultural activities on surface-water quality in this drainage basin. Samples were collected at this site and analyzed for VOCs to determine the effects of wastewater from the $91 \mathrm{st}$ Avenue WWTP.

\section{METHODS OF DATA COLLECTION AND ANALYSIS}

Data were collected and samples were analyzed according to NAWQA standards and protocols with the exception of analytical techniques for some pesticide degradation compounds. For many of the analyses, the concentrations of compounds detected were less than the minimum reporting level (MRL) for the analytical method; these compounds were positively identified in samples, but the concentrations were estimated. Because of these results, numbers of detections of compounds rather than concentrations of compounds are sometimes discussed in this report. VOC data not previously published are presented in the section of this report entitled "Basic Data"; all other environmental data discussed in this report are presented in other reports (Smith and others, 1997; Tadayon and others, 1998 and 1999).

\section{Field and Laboratory Methods}

Koterba and others (1995) and Lapham and others (1995) describe field methods used for NAWQA ground-water sampling. Field methods used for NAWQA surface-water sampling during 1996-98 are described in detail by Shelton (1994). Methods used to collect surface-water samples for VOC analyses are described by Shelton (1997).

Samples collected during 1996-98 were analyzed for pesticides and VOCs at the National Water Quality Laboratory (NWQL) and the USGS Organic Geochemistry Research Laboratory. Water samples were analyzed at the NWQL for 86 pesticides or pesticide-degradation compounds using two different analytical methods (table 5). One method analyzes for 47 pesticides and uses GCMS on elutions from a C-18 column (Zaugg and others, 1995), and the other method analyzes for 39 pesticides (primarily those used in agricultural applications) and uses HPLC on elutions from a Carbopak-B cartridge (Werner and others, 1996). After review of data produced from the HPLC method, the MRLs for some pesticides were increased after December 15, 1997 (W.T. Foreman, chemist, and R.J. Gilliom, hydrologist, USGS, written commun., 1998; table 5, this report). Reported concentrations of some compounds determined from the HPLC method were qualified as estimated because of variable performance during the analytical process (W.T. Foreman, chemist, and R.J. Gilliom, hydrologist, USGS, written commun., 1998; table 5, this report). 
Table 5. Pesticide and pesticide-degradation compound analyses by the National Water Quality Laboratory for surface-water and groundwater samples, Central Arizona Basins study area, 1996-98

[Concentrations are in micrograms per liter; HPLC, high-performance liquid chromatography; GCMS, gas chromatography/mass spectrometry; NA, not applicable; Type: H, herbicide; I, insecticide; DP, degradation compound; F, fungicide]

\begin{tabular}{|c|c|c|c|c|c|}
\hline Pesticide & Type & WATSTORE code & Trade name(s) & Analysis method & $\begin{array}{c}\text { Minimum } \\
\text { reporting level }\end{array}$ \\
\hline Acetochlor & $\mathrm{H}$ & 49260 & Acenit, Guardian, and many other names & GCMS & 0.002 \\
\hline Acifluorfen & $\mathrm{H}$ & 49315 & Blazer, Tackle & HPLC & .035 \\
\hline Alachlor & $\mathrm{H}$ & 46342 & Lasso & GCMS & .002 \\
\hline Aldicarb & I & 49312 & Temik & HPLC & $1,2.550$ \\
\hline Aldicarb sulfone & $\begin{array}{c}\text { DP } \\
\text { (Aldicarb) }\end{array}$ & 49313 & NA & HPLC & $1,2.100$ \\
\hline Aldicarb sulfoxide & $\begin{array}{c}\text { DP } \\
\text { (Aldicarb) }\end{array}$ & 49314 & NA & HPLC & ${ }^{1,2} .021$ \\
\hline Atrazine & $\mathrm{H}$ & 39632 & AAtrex & GCMS & .001 \\
\hline Azinphos-methyl & I & 82686 & Guthion & GCMS & .001 \\
\hline Benfluralin & $\mathrm{H}$ & 82673 & Balan, Benefin & GCMS & .002 \\
\hline Bentazon & $\mathrm{H}$ & 38711 & Basagran & HPLC & .014 \\
\hline Bromacil & $\mathrm{H}$ & 04029 & Bromax 90, Urox B & HPLC & .035 \\
\hline Bromoxynil & $\mathrm{H}$ & 49311 & Buctril, Brominal & HPLC & .035 \\
\hline Butylate & $\mathrm{H}$ & 04028 & Sutan & GCMS & .002 \\
\hline Carbaryl & I & 82680 & Sevin & GCMS & .003 \\
\hline Carbaryl & I & 49310 & Sevin & HPLC & .008 \\
\hline Carbofuran & I & 82674 & Furadan & GCMS & .003 \\
\hline Carbofuran & I & 49309 & Furadan & HPLC & ${ }^{1} .120$ \\
\hline Chloramben & $\mathrm{H}$ & $\begin{array}{c}61188 \\
\text { orig. } 49307\end{array}$ & Amiben & HPLC & ${ }^{1} .420$ \\
\hline Chlorothalonil & $\mathrm{F}$ & 49306 & Bravo & HPLC & $1,2.480$ \\
\hline Chlorpyrifos & I & 38933 & Dursban, Lorsban & GCMS & .004 \\
\hline Clopyralid & $\mathrm{H}$ & 49305 & Lontrel & HPLC & ${ }^{1} .230$ \\
\hline Cyanazine & $\mathrm{H}$ & 04041 & Bladex & GCMS & .004 \\
\hline 2,4-D & $\mathrm{H}$ & 39732 & 2,4-D and many other names & HPLC & ${ }^{1} .150$ \\
\hline Dacthal & $\mathrm{H}$ & 82682 & DCPA, Chlorthal, Clorthal-dimethyl & GCMS & .002 \\
\hline Dacthal mono-acid & $\mathrm{H}$ & 49304 & D. monoacid & HPLC & .017 \\
\hline 2,4-DB & $\mathrm{H}$ & 38746 & Butoxone & HPLC & ${ }^{1} .240$ \\
\hline DDE & $\begin{array}{c}\mathrm{DP} \\
\text { (DDT) }\end{array}$ & 34653 & NA & GCMS & .006 \\
\hline Deethylatrazine & $\begin{array}{c}\text { DP } \\
\text { (Atrazine) }\end{array}$ & 04040 & NA & GCMS & .002 \\
\hline Diazinon & I & 39572 & Diazinon and many other names & GCMS & .002 \\
\hline Dicamba & $\mathrm{H}$ & 38442 & Banvel & HPLC & .035 \\
\hline Dichlobenil & $\mathrm{H}$ & 49303 & Casoron & HPLC & $1,21.200$ \\
\hline Dichlorprop & $\mathrm{H}$ & 49302 & 2,4-DP & HPLC & .032 \\
\hline Dieldrin & I & 39381 & Panoram D-31 & GCMS & .001 \\
\hline 2,6-Diethylaniline & DP (Alachlor) & 82660 & NA & GCMS & .003 \\
\hline Dinoseb & $\mathrm{H}$ & 49301 & Basanite and many other names & HPLC & .035 \\
\hline
\end{tabular}


Table 5. Pesticide and pesticide-degradation compound analyses by the National Water Quality Laboratory for surface-water and groundwater samples, Central Arizona Basins study area, 1996-98-Continued

\begin{tabular}{|c|c|c|c|c|c|}
\hline Pesticide & Type & WATSTORE code & Trade name(s) & Analysis method & $\begin{array}{c}\text { Minimum } \\
\text { reporting level }\end{array}$ \\
\hline Disulfoton & I & 82677 & Disyston and many other names & GCMS & .017 \\
\hline Diuron & $\mathrm{H}$ & 49300 & Diurex and many other names & HPLC & .020 \\
\hline DNOC & I & 49299 & Sinox and many other names & HPLC & $1,2.420$ \\
\hline EPTC & $\mathrm{H}$ & 82668 & Eptam & GCMS & .002 \\
\hline Ethalfluralin & $\mathrm{H}$ & 82663 & Sonalan & GCMS & .004 \\
\hline Ethoprop & I & 82672 & Mocap & GCMS & .003 \\
\hline Fenuron & $\mathrm{H}$ & 49297 & Beet-Kleen & HPLC & .013 \\
\hline Fluometuron & $\mathrm{H}$ & 38811 & Cotoran & HPLC & .035 \\
\hline Fonofos & I & 04095 & Dyfonate & GCMS & .003 \\
\hline alpha-HCH & I & 34253 & Lindane (impurity) & GCMS & .002 \\
\hline gamma-HCH & I & 39341 & Lindane & GCMS & .004 \\
\hline 3-Hydroxycarbofuran & $\begin{array}{c}\text { DP } \\
\text { (Carbofuran) }\end{array}$ & 49308 & NA & HPLC & .014 \\
\hline Linuron & $\mathrm{H}$ & 82666 & Lorox & GCMS & .002 \\
\hline Linuron & $\mathrm{H}$ & 38478 & Lorox & HPLC & .018 \\
\hline MCPA & $\mathrm{H}$ & 38482 & MCPA and many other names & HPLC & ${ }^{1} .170$ \\
\hline MCPB & $\mathrm{H}$ & 38487 & Thistrol & HPLC & ${ }^{1} .140$ \\
\hline Malathion & I & 39532 & Malathion and many other names & GCMS & .005 \\
\hline Methiocarb & I & 38501 & Mesurol & HPLC & .026 \\
\hline Methomyl & I & 49296 & Lannate and many other names & HPLC & .017 \\
\hline Methyl parathion & I & 82667 & Penncap-M & GCMS & .006 \\
\hline Metolachlor & $\mathrm{H}$ & 39415 & Dual & GCMS & .002 \\
\hline Metribuzin & $\mathrm{H}$ & 82630 & Lexone, Sencor & GCMS & .004 \\
\hline Molinate & $\mathrm{H}$ & 82671 & Ordram & GCMS & .004 \\
\hline Napropamide & $\mathrm{H}$ & 82684 & Devrinol & GCMS & .003 \\
\hline Neburon & $\mathrm{H}$ & 49294 & Neburex & HPLC & .015 \\
\hline Norflurazon & $\mathrm{H}$ & 49293 & Evital, Zorial & HPLC & .024 \\
\hline Oryzalin & $\mathrm{H}$ & 49292 & Surflan & HPLC & ${ }^{1} .310$ \\
\hline Oxamyl & I & 38866 & Vydate & HPLC & .018 \\
\hline Parathion & I & 39542 & Alkron and many other names & GCMS & .004 \\
\hline Pebulate & $\mathrm{H}$ & 82669 & Tillam & GCMS & .004 \\
\hline Pendimethalin & $\mathrm{H}$ & 82683 & Prowl & GCMS & .004 \\
\hline cis-Permethrin & I & 82687 & Ambush, Pounce & GCMS & .005 \\
\hline Phorate & I & 82664 & Thimet and many other names & GCMS & .002 \\
\hline Picloram & $\mathrm{H}$ & 49291 & Tordon & HPLC & .050 \\
\hline Prometon & $\mathrm{H}$ & 04037 & Pramitol & GCMS & .018 \\
\hline Pronamide & $\mathrm{H}$ & 82676 & Kerb & GCMS & .003 \\
\hline
\end{tabular}


Table 5. Pesticide and pesticide-degradation compound analyses by the National Water Quality Laboratory for surface-water and groundwater samples, Central Arizona Basins study area, 1996-98-Continued

\begin{tabular}{|c|c|c|c|c|c|}
\hline Pesticide & Type & WATSTORE code & Trade name(s) & Analysis method & $\begin{array}{c}\text { Minimum } \\
\text { reporting level }\end{array}$ \\
\hline Propachlor & $\mathrm{H}$ & 04024 & Ramrod & GCMS & .007 \\
\hline Propanil & $\mathrm{H}$ & 82679 & Stampede & GCMS & .004 \\
\hline Propargite & I & 82685 & Comite, Omite & GCMS & .013 \\
\hline Propham & $\mathrm{H}$ & 49236 & IPC & HPLC & .035 \\
\hline \multicolumn{6}{|c|}{ See footnotes at end of table. } \\
\hline Propoxur & I & 38538 & Baygon & HPLC & .035 \\
\hline Silvex & $\mathrm{H}$ & 39762 & $2,4,5-\mathrm{TP}$ & HPLC & .021 \\
\hline Simazine & $\mathrm{H}$ & 04035 & Princep, Aquazine & GCMS & .005 \\
\hline $2,4,5-\mathrm{T}$ & $\mathrm{H}$ & 39742 & Line Rider and many other names & HPLC & .035 \\
\hline Tebuthiuron & $\mathrm{H}$ & 82670 & Spike, Graslan & GCMS & .010 \\
\hline Terbacil & $\mathrm{H}$ & 82665 & Sinbar & GCMS & .007 \\
\hline Terbufos & I & 82675 & Counter & GCMS & .013 \\
\hline Thiobencarb & $\mathrm{H}$ & 82681 & Bolero & GCMS & .002 \\
\hline Triallate & $\mathrm{H}$ & 82678 & Far-Go & GCMS & .001 \\
\hline Triclopyr & $\mathrm{H}$ & 49235 & Garlon & HPLC & ${ }^{1} .250$ \\
\hline Trifluralin & $\mathrm{H}$ & 82661 & Treflan and many other names & GCMS & .002 \\
\hline
\end{tabular}

${ }^{1}$ Minimum reporting level was increased after December 15, 1997.

${ }^{2}$ If compound is in sample, concentration is estimated.

In addition to analyses completed by the NWQL, some samples were analyzed for pesticide-degradation compounds by the USGS Organic Geochemistry Research Laboratory in Lawrence, Kansas (table 6). Analytical methods included elutions from a C-18 column and GCMS and HPLC (Hostetler and Thurman, 1999).

Table 6. Pesticide-degradation compound analyses by the U.S. Geological Survey, Organic Geochemistry Research Group, Lawrence, Kansas, for ground-water and surface-water samples, Central Arizona Basins study area, 1996-98

[HPLC, high-performance liquid chromatography; GCMS, gas chromatography/mass spectrometry]

\begin{tabular}{llc}
\hline $\begin{array}{c}\text { Pesticide-degradation } \\
\text { compound }\end{array}$ & Pesticide source & $\begin{array}{c}\text { Analytical } \\
\text { method }\end{array}$ \\
\hline Acetochlor ESA & Acetochlor & HPLC \\
Alachlor ESA & Alachlor & HPLC \\
Metolachlor ESA & Metolachlor & HPLC \\
Deisopropylatrazine & Atrazine & GCMS \\
3,4-Dichloroaniline & Propanil, Linuron & GCMS \\
\hline
\end{tabular}

Data for VOCs were produced by three different analytical methods following sampling in 1996-98 (table 7). Purge-and-trap capillary-column GCMS was used to determine 60 VOCs at a MRL of $0.2 \mu \mathrm{g} / \mathrm{L}$ (Rose and Schroeder, 1995) in samples collected prior to March 27, 1996. Water samples collected after March 27, 1996, also were analyzed by purge-and-trap capillary-column GCMS according to Connor and others (1998). The analytical method used after March 27, 1996, provides reliable evidence of the presence of an analyte and is suitable for reporting concentrations at submicrogram-per-liter levels for 87 VOCs. After May 1997, vinyl acetate was eliminated from the analysis because of poor analytical performance. The method also allows for reporting estimated concentrations of other tentatively identified VOCs. The main difference between data obtained before October 1, 1996, and data obtained after this date is how low-level detections of VOCs that were affected by laboratory contaminants were evaluated or censored in relation to internal laboratory blanks. 
Table 7. Volatile organic compound analyses by the National Water Quality Laboratory for ground-water and surface-water samples, Central Arizona Basins study area, 1996-98

[WATSTORE, National Water Data Storage Retrieval System]

\begin{tabular}{|c|c|c|c|c|}
\hline \multirow[b]{2}{*}{ Volatile organic compound } & \multirow[b]{2}{*}{ WATSTORE Code } & \multicolumn{3}{|c|}{ Minimum reporting level } \\
\hline & & Prior to March 27, 1996 & March 27 to June 9, 1997 & $\begin{array}{l}\text { June 10, 1997, to } \\
\text { September 30, } 1998\end{array}$ \\
\hline Dibromomethane & 30217 & 0.2 & 0.1 & 0.05 \\
\hline Bromodichloromethane & 32101 & .2 & .1 & .048 \\
\hline Dibromochloromethane & 32105 & .2 & .1 & .182 \\
\hline Trichloromethane & 32106 & .2 & .05 & .052 \\
\hline Methylbenzene & 34010 & .2 & .05 & .038 \\
\hline Chlorobenzene & 34301 & .2 & .05 & .028 \\
\hline Tetrachloroethene & 34475 & .2 & .05 & .038 \\
\hline 1,4-Dichlorobenzene & 34571 & .2 & .05 & .05 \\
\hline Carbon disulfide & 77041 & --- & .05 & .08 \\
\hline Bromochloromethane & 77297 & .2 & .1 & .044 \\
\hline Tetrachloromethane & 32102 & .2 & .05 & .088 \\
\hline 1,2-Dichloroethane & 32103 & .2 & .05 & .134 \\
\hline Tribromomethane & 32104 & .2 & .2 & .104 \\
\hline Benzene & 34030 & .2 & .05 & .032 \\
\hline 2-Propenal & 34210 & --- & 2. & 1.43 \\
\hline 2-Propenenitrile & 34215 & --- & 2. & 1.23 \\
\hline Chloroethane & 34311 & .2 & .1 & .120 \\
\hline Ethylbenzene & 34371 & .2 & .05 & .03 \\
\hline 1,1,1,2,2,2-Hexa-chloroethane & 34396 & --- & .05 & .362 \\
\hline Bromomethane & 34413 & .2 & .1 & .148 \\
\hline Chloromethane & 34418 & .2 & .2 & .254 \\
\hline Dichloromethane & 34423 & .2 & .1 & .382 \\
\hline Trichlorofluoromethane & 34488 & .2 & .1 & .092 \\
\hline 1,1-Dichloroethane & 34496 & .2 & .05 & .066 \\
\hline 1,1-Dichloroethene & 34501 & .2 & .1 & .044 \\
\hline 1,1,1-Trichloroethane & 34506 & .2 & .05 & .032 \\
\hline $1,1,2$-Trichloroethane & 34511 & .2 & .1 & .064 \\
\hline 1,1,2,2-Tetrachloroethane & 34516 & .2 & .1 & .132 \\
\hline 1,2-Dichlorobenzene & 34536 & .2 & .05 & .048 \\
\hline 1,2-Dichloropropane & 34541 & .2 & .05 & .068 \\
\hline trans-1,2-Dichloroethene & 34546 & .2 & .05 & .032 \\
\hline 1,2,4-Trichlorobenzene & 34551 & .2 & .2 & .188 \\
\hline 1,3-Dichlorobenzene & 34566 & .2 & .05 & .054 \\
\hline Dichlorodifluoromethane & 34668 & .2 & .2 & .096 \\
\hline Naphthalene & 34696 & .2 & .2 & .250 \\
\hline trans-1,3-Dichloropropene & 34699 & .2 & .1 & .134 \\
\hline cis-1,3-Dichloropropene & 34704 & .2 & .1 & .092 \\
\hline Chloroethene & 39175 & .2 & .1 & .112 \\
\hline Trichloroethene & 39180 & .2 & .05 & .038 \\
\hline Hexachlorobutadiene & 39702 & .2 & .2 & .142 \\
\hline Methyl acrylate & 49991 & --- & 2. & 612 \\
\hline 1,2,3,4-Tetramethylbenzene & 49999 & --- & .05 & .230 \\
\hline $1,2,3,5$-Tetramethylbenzene & 50000 & --- & .05 & .240 \\
\hline
\end{tabular}




\begin{tabular}{|c|c|c|c|c|}
\hline Bromoethene & 50002 & --- & .1 & .1 \\
\hline Ethyl-tert-butyl ether & 50004 & --- & .1 & .054 \\
\hline tert-Amyl methyl ether & 50005 & --- & .1 & .112 \\
\hline trans-1,4-Dichloro-2-butene & 73547 & --- & 5. & .692 \\
\hline Ethyl methacrylate & 73570 & --- & 1.0 & .278 \\
\hline Vinyl acetate & 77057 & --- & 5. & --- \\
\hline cis-1,2-Dichloroethene & 77093 & .2 & .05 & .038 \\
\hline 2-Hexanone & 77103 & --- & 5. & .746 \\
\hline Ethenylbenzene & 77128 & .2 & .05 & .042 \\
\hline 1,2-Dimethylbenzene & 77135 & --- & .05 & .064 \\
\hline 1,1-Dichloropropene & 77168 & .2 & .05 & .026 \\
\hline 2,2-Dichloropropane & 77170 & .2 & .05 & .078 \\
\hline 1,3-Dichloropropane & 77173 & .2 & .05 & .116 \\
\hline 2-Ethyltoluene & 77220 & --- & .05 & .10 \\
\hline 1,2,3-Trimethylbenzene & 77221 & --- & .05 & .124 \\
\hline 1,2,4-Trimethylbenzene & 77222 & .2 & .05 & .056 \\
\hline (1-Methylethyl)benzene & 77223 & .2 & .05 & .032 \\
\hline n-Propylbenzene & 77224 & .2 & .05 & .042 \\
\hline 1,3,5-Trimethylbenzene & 77226 & .2 & .05 & .044 \\
\hline 1-Chloro-2-methylbenzene & 77275 & .2 & .05 & .042 \\
\hline 1-Chloro-4-methylbenzene & 77277 & .2 & .05 & .056 \\
\hline n-Butylbenzene & 77342 & .2 & .05 & .186 \\
\hline (1-Methylpropyl)benzene & 77350 & .2 & .05 & .048 \\
\hline (1,1-Dimethylethyl)benzene & 77353 & .2 & .05 & .096 \\
\hline 1-Isopropyl-4-methylbenzene & 77356 & .2 & .05 & .110 \\
\hline Iodomethane & 77424 & --- & .05 & .076 \\
\hline 1,2,3-Trichloropropane & 77443 & .2 & .2 & .07 \\
\hline 1,1,1,2-Tetrachloroethane & 77562 & .2 & .05 & .044 \\
\hline 1,2,3-Trichlorobenzene & 77613 & .2 & .2 & .266 \\
\hline 1,2-Dibromomethane & 77651 & .2 & .1 & .036 \\
\hline 1,1,2-Trichloro-1,2,2-trifluoroethane & 77652 & .2 & .05 & .032 \\
\hline Methyl tert-butyl ether & 78032 & .2 & .1 & .112 \\
\hline 3-Chloro-1-propene & 78109 & --- & .1 & .196 \\
\hline 4-Methyl-2-pentanone & 78133 & --- & 5. & .374 \\
\hline Xylenes, Total & 81551 & .2 & 1 & 1--- \\
\hline Acetone & 81552 & --- & 5. & 4.90 \\
\hline Bromobenzene & 81555 & .2 & .05 & .036 \\
\hline Diethyl ether & 81576 & --- & .1 & .170 \\
\hline Diisopropyl ether & 81577 & --- & .1 & .098 \\
\hline Methyl acrylonitrile & 81593 & --- & 2. & .570 \\
\hline 2-Butanone & 81595 & --- & 5. & 1.65 \\
\hline Methyl methacrylate & 81597 & --- & 1. & .35 \\
\hline Tetrahydrofuran & 81607 & --- & 5. & 1.15 \\
\hline 1,2-Dibromo-3-chloropropane & 82625 & .2 & .5 & .214 \\
\hline $\begin{array}{l}\text { 1,3-Dimethylbenzene and } \\
\text { 1,4-Dimethylbenzene }\end{array}$ & 85795 & --- & .05 & .064 \\
\hline
\end{tabular}


From March 27, 1996, to October 1, 1996, environmental samples found to contain laboratoryderived contaminants were censored (that is, reported as less than the detection limit) regardless of the magnitude of daily laboratory-blank values. Additional laboratory-blank samples were incorporated into the analytical sequence after October 1, 1996, to help distinguish between true low-level environmental concentrations and low-level environmental concentrations caused by laboratory background contamination. Laboratory contaminants that were censored were benzene, ethylbenzene, methylbenzene, xylenes, 1,4-dichlorobenzene, dichloromethane, acetone, and carbon disulfide. The automatic censoring used from March 27, 1996, to October 1, 1996, resulted in an underestimate of the occurrence of the censored compounds in environmental samples. Detections of benzene and carbon disulfide were reported for groundwater samples during this time period.

\section{Quality Assurance}

Quality-control samples were collected and analyzed as part of the ground-water and surface-water data collection. Evaluation of the quality-control samples for pesticides and VOCs in ground water indicated that the environmental data generally are acceptable. Some detections of VOCs are considered to be the result of sample contamination. Evaluation of quality-control samples for pesticides in surface water indicated that the associated environmental data are acceptable. The quality-control samples for VOCs in surface water are inadequate to determine whether the associated environmental data are acceptable.

The quantity of quality-control samples was not sufficient to characterize the quality of environmental data throughout the entire sampling period. A detailed discussion of the quality assurance is included in the section entitled "Quality-Assurance Information."

\section{Statistical Methods}

Concentrations of organic compounds detected in ground-water and surface-water samples generally were below the MRLs or qualified as estimated. Interpretation of the data is based primarily on the number of detections and not the reported concentrations.
The Kruskal-Wallis test for ordered categorical responses was applied (Helsel and Hirsch, 1992; p. 382) to compare results among the SUSs. For this test, laboratory results were categorized into three responses-below the reporting limit, above the reporting limit, and estimated (the compound was detected in the sample, but the concentration was not considered accurate). The Kruskal-Wallis test statistic was computed, and the results indicated whether the null hypothesis, which is that the proportion of data in each response category was the same for each groundwater study, was considered valid at the 95-percent confidence level. If the Kruskal-Wallis test indicated that there were differences between the SUSs, the Tukey test was applied to identify the specific differences.

\section{OCCURRENCE AND DISTRIBUTION OF PESTICIDES AND VOLATILE ORGANIC COMPOUNDS IN GROUND WATER AND SURFACE WATER AND RELATION TO LAND USE}

The occurrence and distribution of pesticides and VOCs in ground water and surface water from the areas studied during 1996-98 range from no detections to several detections in localized areas. The basin that is considered minimally developed, the Sierra Vista subbasin, had the fewest detections of pesticides and VOCs in ground water and of pesticides in surface water. Basins that are considered developed, the Upper Santa Cruz Basin and the West Salt River Valley, have some large localized areas that have water quality that is affected by human activities. The largest number of detections of pesticides and VOCs occurred in ground water and surface water in the agricultural area in the western part of the West Salt River Valley.

\section{Pesticides}

Detections of pesticides in ground water and surface water in the CAZB indicate the effects on water quality of both agricultural and urban pesticides used on the land surface. The largest number of detections of pesticides occurred in shallow ground water beneath the agricultural area in the West Salt River Valley (fig. 5). Fewer detections were found in ground water from the SUSs. 


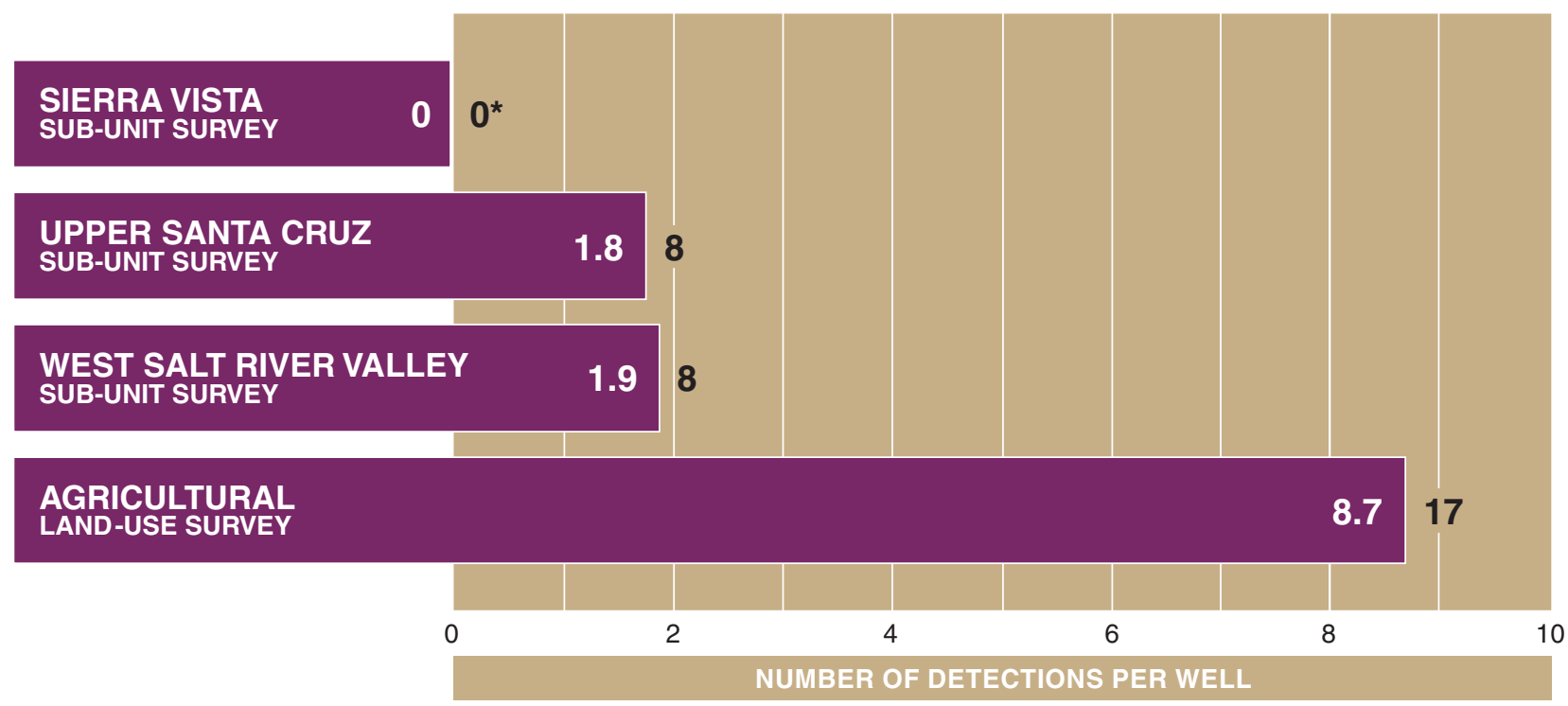

Figure 5. Number of pesticide detections per well, Central Arizona Basins study area, 1996-98. Detections per well equal number of pesticide detections divided by number of samples with detections. Numbers in black are the number of samples with pesticides detected. * No samples for HPLC analysis were collected in the Sierra Nevada sub-unit survey.

\section{Minimally Developed Basin}

Ground-water and surface-water samples from the minimally developed Sierra Vista subbasin did not contain any of the 47 or 86 pesticides for which these samples were analyzed, respectively (table 8). Quality control samples indicate that sample contamination was not an issue for ground-water samples from this basin. These results indicate that the water quality in this basin is not severely affected by sources of pesticides.

When pesticide detections for the minimally developed basin are compared with the results from other ground-water studies in the CAZB, it is apparent that the distribution of pesticide detections corresponds to the relative amounts of urban and agricultural landuse in each basin (fig. 5). The minimally developed basin had the fewest number of detections per well, and the other two basins had the most detections. The number of detections per well is determined by dividing the number of pesticide detections from all samples in a SUS or LUS survey by the number of samples with detections. Samples from the LUS had the highest number of detections of pesticides per well. This result corresponds with the intensive agricultural land use and shallow depths to ground water in the LUS area compared to conditions in the SUS areas. Statistical comparisons of pesticide detections for the
SUSs indicated that the results for the Sierra Vista SUS were different than those for the Upper Santa Cruz Basin and the West Salt River Valley SUSs at the 95- and 90-percent confidence levels, respectively. No difference was identified between pesticide results for the Upper Santa Cruz Basin SUS and results for the West Salt River Valley SUS. Comparison between the SUSs and the agricultural LUS was not deemed appropriate because of the large difference in available samples and the difference in well types.

Of the six surface-water samples collected for analyses of pesticides from the San Pedro River at Charleston (09471000), two samples represented highflow conditions and four samples represented low-flow conditions. A sample collected on June 24, 1997, contained DDE at an estimated concentration (below the MRL) of $0.0007 \mu \mathrm{g} / \mathrm{L}$. Samples collected at sites on the Buckeye Canal and the Hassayampa River on June 17 and June 18, 1997, respectively, also had detectable concentrations of DDE. Because the same sampling equipment was used at all sites, the detection of DDE at San Pedro on June 24 could represent carryover from samples collected on June 17 and June 18. Because of the possibility of carryover contamination, the detection of DDE in the San Pedro River will be considered a nondetection in this report. This decision is supported by the lack of detections of DDE in bed-sediment and fish-tissue samples collected from the San Pedro River (Gebler, 2000). 
Table 8. Number of pesticides detected in ground-water and surface-water samples, Sierra Vista subbasin, Central Arizona Basins study area, 1996-97

[Dashes indicate that the information is not applicable]

\begin{tabular}{lcccccc}
\hline & & \multicolumn{4}{c}{ Pesticides } \\
\cline { 4 - 7 } $\begin{array}{c}\text { Ground-water study name or } \\
\text { surface-water site name }\end{array}$ & $\begin{array}{c}\text { Medium } \\
\text { sampled }\end{array}$ & $\begin{array}{c}\text { Surface-water } \\
\text { site number }\end{array}$ & $\begin{array}{c}\text { Samples } \\
\text { collected }\end{array}$ & Detections & $\begin{array}{c}\text { Samples with } \\
\text { detectable } \\
\text { concentrations }\end{array}$ & $\begin{array}{c}\text { Compounds } \\
\text { detected }\end{array}$ \\
\hline Sierra Vista subbasin & ground water & -- & 19 & 10 & 0 & 0 \\
San Pedro River at Charleston & surface water & 09471000 & 6 & 0 & 0 & 0 \\
\hline
\end{tabular}

${ }^{1}$ Analyses of pesticides using the HPLC method were not completed.

The results from ground-water and surface-water samples from the Sierra Vista subbasin are important because they represent water quality in an area of the CAZB where pesticides generally have not been used on the land surface. Data like these have not been available before this study, and they provide important baseline information for future monitoring in this basin.

\section{Developed Basins}

Ground-water and surface-water samples from the Upper Santa Cruz Basin and the West Salt River Valley were used to characterize areas in which water quality is affected by human activities. In these basins, ground water and surface water are affected by pesticides currently used and pesticides used in the past.

Pesticides detected in ground water and surface water can be attributed to present-day urban and agricultural land use (U.S. Geological Survey, 1999); other pesticides can be attributed to the historical land use that previously dominated the landscape.

Concentrations of pesticides detected in ground water in these basins were below current water-quality limits established by the USEPA (1996) and the State of Arizona (1996); some concentrations of pesticides in surface water were above the water-quality limits (table 9). The detections indicate that the ground water and surface water in these areas are susceptible to contamination by human activities, and the results may provide information on the contaminant sources and transport processes.

\section{Upper Santa Cruz Basin}

Agricultural activities have been present in the Upper Santa Cruz Basin since the early 1900s (Water Resources Research Center, 1999), but the effects of these activities on ground water are not widely distributed. Detections of pesticides in the Santa Cruz River near Nogales indicate that urban activities are affecting the surface-water quality because the pesticides detected are characterized as "urban insecticides." A few detections of pesticides in surfacewater samples were higher than established waterquality limits of the USEPA and the State of Arizona (table 9).

Samples from 8 wells (28 percent; table 10) in the Upper Santa Cruz Basin (fig. 6) contained 5 of the 86 pesticides analyzed. Deethylatrazine was the most frequently detected compound and was detected in 6 samples; atrazine was detected in 5 samples; and prometon, 2,4-D, and diuron were detected in 1 sample each.

Samples from five wells-SC5, SC7, SC8, SC12, and $\mathrm{SC} 16$ - contained atrazine and deethylatrazine. Deethylatrazine is a degradation compound of the herbicide atrazine. Atrazine is a selective herbicide that controls broadleaf and grassy weeds in agricultural areas and also is applied as a nonselective herbicide in nonagricultural areas (Extension Toxicology Network, 1996). This compound is highly persistent in soil and is moderately to highly mobile in the subsurface. 
Table 9. Maximum Contaminant Levels, human health advisory limits, aquifer water-quality standards, and surface-water standards for pesticides detected in ground-water and surface-water samples, Central Arizona Basins study area, 1996-98

[Constituents are dissolved and are reported in micrograms per liter; HAL, U.S. Environmental Protection Agency established human health advisory level for drinking water; MCL, Maximum Contaminant Level; E, concentration is estimated; dashes indicate no data]

\begin{tabular}{|c|c|c|c|c|}
\hline \multirow[b]{2}{*}{ Constituent } & \multicolumn{2}{|c|}{ Concentration range of detections } & \multicolumn{2}{|c|}{ U.S. Environmental Protection Agency ${ }^{1}$} \\
\hline & Ground water & Surface water & $\mathrm{HAL}^{3}$ & Primary MCL \\
\hline Atrazine & E0.00267-.079 & $0.00486-.0366$ & --- & 3 \\
\hline Chlorpyrifos & E.0035 & $.0088-.154$ & 20 & --- \\
\hline Cyanazine & --- & $.007-.635$ & $5_{1}$ & --- \\
\hline DDE & Е.0012-.0076 & $.00191-.012$ & --- & --- \\
\hline Dieldrin $^{6}$ & $.006-.033$ & .0103 & --- & --- \\
\hline Disulfoton & --- & .826 & .3 & --- \\
\hline Diuron & $.04-\mathrm{E} 5.53$ & --- & 10 & --- \\
\hline Lindane (gamma-HCH) & --- & $.006-.030$ & .2 & .2 \\
\hline Metribuzin & .007 & $.0169-.153$ & 100 & --- \\
\hline Prometon & E.0024-.108 & $.0057-.0378$ & ${ }^{7} 100$ & --- \\
\hline Simazine & E.0014-.0662 & $.00554-.0365$ & --- & 4 \\
\hline Terbacil & E.015 & --- & 90 & --- \\
\hline Trifluralin & Е.0027-.0089 & $.00176-.097$ & 5 & --- \\
\hline
\end{tabular}

\begin{tabular}{|c|c|c|c|c|c|}
\hline \multirow[b]{3}{*}{ Constituent } & \multicolumn{5}{|c|}{ State of Arizona } \\
\hline & \multirow[b]{2}{*}{$\begin{array}{l}\text { Aquifer } \\
\text { water-quality } \\
\text { standard }\end{array}$} & \multicolumn{4}{|c|}{ Surface water } \\
\hline & & Fish consumption & $\begin{array}{l}\text { Agricultural } \\
\text { livestock } \\
\text { watering }\end{array}$ & $\begin{array}{c}\text { Agricultural } \\
\text { irrigation }\end{array}$ & $\begin{array}{l}\text { Aquatic and } \\
\text { wildlife }^{4}\end{array}$ \\
\hline Atrazine & --- & --- & --- & --- & --- \\
\hline Chlorpyrifos & --- & --- & --- & --- & --- \\
\hline Cyanazine & --- & --- & --- & --- & --- \\
\hline DDE & --- & 0.0006 & 0.001 & 0.001 & 0.02 \\
\hline Dieldrin 6 & --- & .0002 & ${ }^{7} .003$ & ${ }^{7} .003$ & .002 \\
\hline Disulfoton & --- & --- & --- & --- & --- \\
\hline Diuron & --- & --- & --- & --- & --- \\
\hline Lindane (gamma-HCH) & 0.2 & .02 & --- & --- & .61 \\
\hline Metribuzin & --- & --- & --- & --- & --- \\
\hline Prometon & --- & --- & --- & --- & --- \\
\hline Simazine & 4 & --- & --- & --- & --- \\
\hline Terbacil & --- & --- & --- & --- & --- \\
\hline Trifluralin & --- & --- & --- & --- & --- \\
\hline
\end{tabular}


Table 10. Number of pesticides detected in ground-water and surface-water samples in the Upper Santa Cruz Basin, Central Arizona Basins study area, 1996-98

[Dashes indicate that the information is not applicable]

\begin{tabular}{|c|c|c|c|c|c|c|}
\hline \multirow[b]{2}{*}{$\begin{array}{l}\text { Ground-water study name } \\
\text { or surface-water site name }\end{array}$} & \multirow[b]{2}{*}{$\begin{array}{l}\text { Medium } \\
\text { sampled }\end{array}$} & \multirow[b]{2}{*}{$\begin{array}{l}\text { Surface-water } \\
\text { site number }\end{array}$} & \multicolumn{4}{|c|}{ Pesticides } \\
\hline & & & $\begin{array}{l}\text { Samples } \\
\text { collected }\end{array}$ & Detections & $\begin{array}{l}\text { Samples with } \\
\text { detectable } \\
\text { concentrations }\end{array}$ & $\begin{array}{c}\text { Compounds } \\
\text { detected }\end{array}$ \\
\hline Upper Santa Cruz sub-unit survey & ground water & --- & 29 & 14 & 8 & 5 \\
\hline $\begin{array}{l}\text { Nogales Wash near the Nogales } \\
\text { International Wastewater Treatment Plant }\end{array}$ & surface water & 312314110565601 & 2 & 2 & 2 & 1 \\
\hline Santa Cruz River near Rio Rico, Arizona & surface water & 312809110592801 & 2 & 11 & 2 & 6 \\
\hline Santa Cruz River at Santa Gertrudis Lane & surface water & 313343110024701 & 2 & 11 & 2 & 6 \\
\hline Santa Cruz River at Tubac, Arizona & surface water & 09481740 & 2 & 9 & 2 & 6 \\
\hline
\end{tabular}

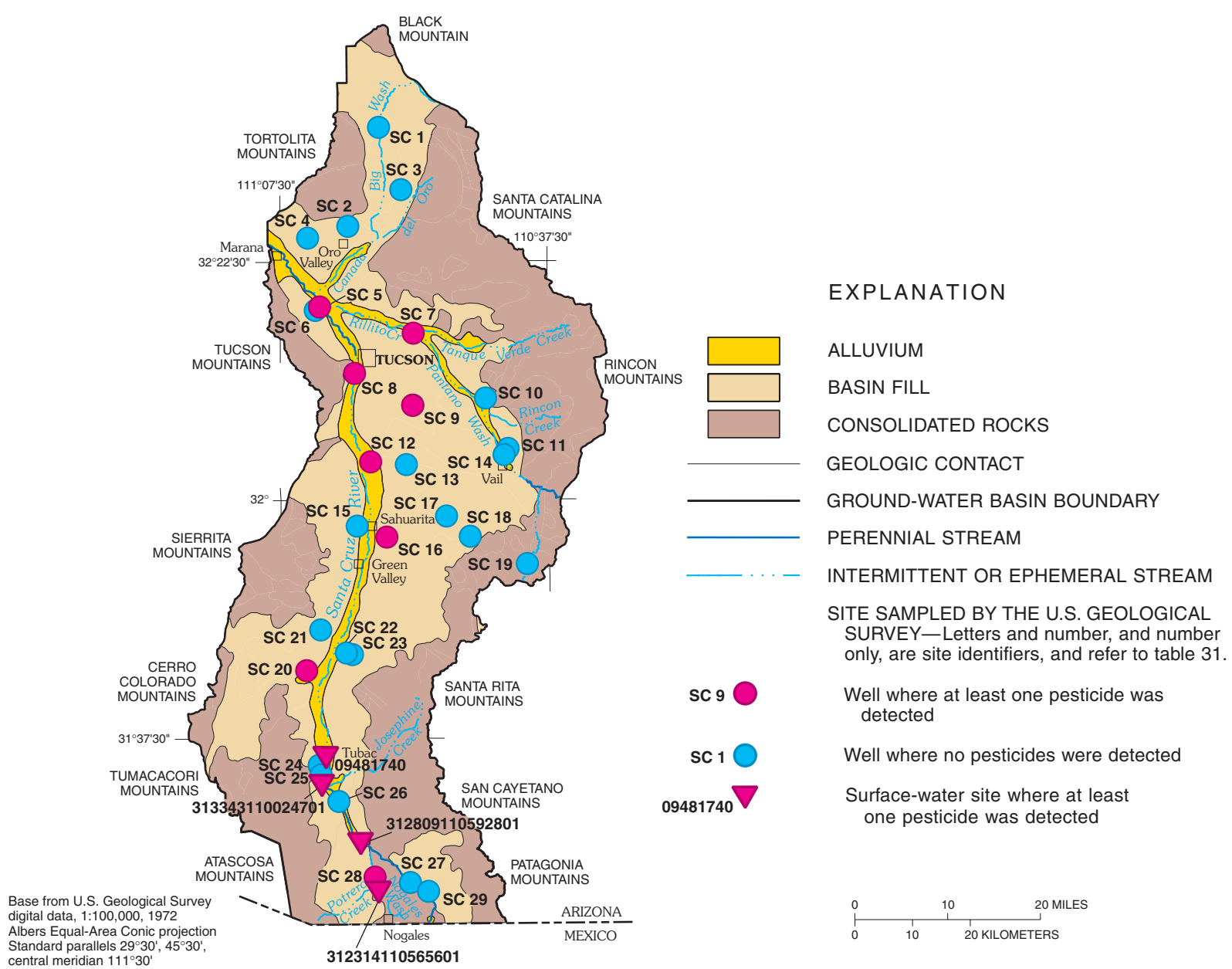

Figure 6. Locations of ground-water and surface-water sites where samples were collected for pesticide analyses, Upper Santa Cruz Basin, Central Arizona Basins study area. 
Detections of atrazine and its degradation compounds, including deethylatrazine, can be expected where this compound is used, especially in areas with shallow depths to ground water and significant recharge (Extension Toxicology Network, 1996). Wells $\mathrm{SC} 5, \mathrm{SC} 7$, and SC8 are in areas of historic agriculture, and agricultural activities have resulted in elevated concentrations of calcium, potassium, alkalinity, and dissolved solids (Coes and others, 2000). Pesticides detected in samples from these wells probably are the result of past applications in these areas. Wells SC12 and SC16 are near areas of agricultural activities, but these activities are not as close to the wells as the historical agricultural areas are to wells SC5, SC7, and SC8. The land surrounding SC12 has been used only to graze cattle; however, the well is about 1 mi east of the Santa Cruz River, which could provide transport of pesticides from upgradient agricultural areas. SC16 is in a newly developed area originally used for rangeland. The only known historical agricultural activities are downgradient from this well. Prometon, 2,4-D, and diuron are considered to be primarily from urban applications, although diuron was used on crops in agricultural areas in 1996 (table 2).

Surface-water samples were collected during lowflow conditions at three sites along the Santa Cruz River and at one site on Nogales Wash, which is a tributary of the Santa Cruz River (fig. 6 and table 10). Seven of the 86 pesticides analyzed were detected in these surface-water samples. Prometon was detected at three of the four sites-Nogales Wash, the Santa Cruz River at Santa Gertrudis Lane, and the Santa Cruz River at Tubac. Chlorpyrifos, lindane, malathion, diazinon, and carbaryl were detected at the three sites along the Santa Cruz River but not at Nogales Wash. The concentration of lindane in the two samples from the Santa Cruz River near Rio Rico exceeded the State of Arizona surface-water standard for fish consumption $(0.02 \mu \mathrm{g} / \mathrm{L}$; table 9). Ethalfluralin was detected at the Santa Cruz River near Rio Rico. Pesticides detected at these sites that are identified as "urban insecticides" (U.S. Geological Survey, 1999) are prometon, diazinon, cabaryl, malathion, and chlorpyrifos. Detections of these insecticides indicate that urban activities near Nogales are affecting surface-water quality in this area.

\section{West Salt River Valley}

Ground-water and surface-water samples from the West Salt River Valley contained more detectable pesticides than samples from any other area sampled in the CAZB during 1996-98. This basin has the most complex combination of land-use types in the CAZB, and the occurrence and distribution of pesticides reflects that combination. The pesticides detected are used in both agricultural and urban land uses. Detections were most numerous in samples of the shallow ground water from the agricultural area in the southwestern part of the basin. Seasonal patterns of pesticides in surface water at two sites in the agricultural area reflect the timing of application of these pesticides. Reuse of water in this arid area contributes to the transport of pesticides from the urban to the agricultural areas.

\section{Ground Water}

A variety of pesticides were detected in the two ground-water studies completed in the West Salt River Valley. Twenty-three percent of the 35 wells sampled for the SUS had detectable concentrations of pesticides (table 11). At least one pesticide was detected in samples from each of the nine wells in the LUS collected in August 1997 and February 1998. Concentrations of pesticides in ground water did not exceed limits established by the USEPA or the State of Arizona. Detections of pesticides in samples from the LUS wells were more numerous than detections in samples from any other group of wells in the CAZB during 1996-98. The ground-water quality in the West Salt River Valley generally represents conditions after large increases in population and agricultural and urban land use.

Eight of 86 pesticides analyzed were detected in water from 8 of the 35 wells sampled for the West Salt River Valley SUS. Numbers of detections for wells in the SUS varied throughout the basin, and pesticides were detected most frequently in samples from the southern part of the basin (fig. 7). The most frequently detected compound was deethylatrazine, which was detected in samples from 4 wells; atrazine, s-ethyl dipropylthiocarbamate (EPTC), simazine, and prometon were detected in samples from 2 wells; and DDE, acetochlor, and triallate each were detected in samples from 1 well. 
Table 11. Number of pesticides detected in ground-water and surface-water samples, West Salt River Valley, Central Arizona Basins study area, 1996-98

[Dashes indicate that the information is not applicable. Data for agricultural land-use study represent two sampling efforts—August 1997 and February 1998 ]

\begin{tabular}{|c|c|c|c|c|c|c|}
\hline \multirow[b]{2}{*}{$\begin{array}{l}\text { Ground-water study name or } \\
\text { surface-water site name }\end{array}$} & \multirow[b]{2}{*}{$\begin{array}{l}\text { Medium } \\
\text { sampled }\end{array}$} & \multirow[b]{2}{*}{$\begin{array}{l}\text { Surface-water } \\
\text { site number }\end{array}$} & \multicolumn{4}{|c|}{ Pesticides } \\
\hline & & & $\begin{array}{l}\text { Samples } \\
\text { collected }\end{array}$ & Detections & $\begin{array}{c}\text { Samples with } \\
\text { detectable } \\
\text { concentrations }\end{array}$ & $\begin{array}{l}\text { Compounds } \\
\text { detected }\end{array}$ \\
\hline $\begin{array}{l}\text { West Salt River Valley sub-unit } \\
\text { survey }\end{array}$ & Ground water & --- & 35 & 15 & 8 & 8 \\
\hline Agricultural land-use study & Ground water & --- & 18 & 78 & 17 & 10 \\
\hline Gila River at Buckeye Canal & Surface water & & 31 & 210 & 31 & 23 \\
\hline $\begin{array}{l}\text { Gila River above diversions at } \\
\text { head of Buckeye Canal }\end{array}$ & & 09513990 & & & & \\
\hline Buckeye Canal near Avondale & & 09514000 & & & & \\
\hline Gila River at Estrella Parkway & & 09514100 & & & & \\
\hline Hassayampa River near Arlington & Surface water & 09517000 & 40 & 317 & 38 & 26 \\
\hline
\end{tabular}

Deethylatrazine and atrazine were detected in samples from SUS wells in agricultural and nonagricultural areas. The detection of pesticides is attributed to characteristics of the pesticide and hydrogeologic conditions, as well as to land use. Atrazine is persistent in the subsurface (Extension Toxicology Network, 1996). The depths to water in 3 of the 4 wells where deethylatrazine was detected were $65 \mathrm{ft}$ or less (Edmonds and Gellenbeck, 2002). Depth to water was more than $400 \mathrm{ft}$ at one well. The combination of persistence and shallow depths to ground water probably contributed to these detections.

EPTC, simazine, and prometon are not as persistent in the subsurface as atrazine (Extension Toxicology Network, 1996; U.S. Department of Agriculture, 1995); therefore, the detection of these compounds may depend on when samples are collected. EPTC was detected in wells in agricultural areas, but simazine and prometon were detected in wells in an urban area. EPTC is used as a selective preemergent herbicide for control of grassy weeds, perennial weeds, and some broadleaf weeds in a variety of crops (Extension Toxicology Network, 1996). EPTC has a low persistence in the soil and a half-life of less than one week (Extension Toxicology Network, 1996). Sampling must have occurred close to the time of application for this compound to have been detected in the ground water. Simazine is applied in both agricultural and nonagricultural settings to control broadleaf weeds and annual grasses (Extension Toxicology Network, 1996). Prometon is applied in nonagricultural settings to control perennial broadleaf and grassy weeds (U.S. Department of Agriculture, 1995). Both compounds are mobile in the subsurface, especially in sandy loam soils (U.S. Department of Agriculture, 1995).

The detections of pesticides in nine wells in the agricultural LUS area in the southwestern part of the West Salt River Valley most clearly reflect the effects of present agricultural activities on ground-water quality, although the detection of some compounds is considered a consequence of urban activities (Edmonds and Gellenbeck, 2002). At least one pesticide was detected in samples from each of the nine wells (fig. 7). Twelve of the 86 pesticides analyzed were detected in samples from 8 of the 9 wells for the LUS in August 1997. Six of the 86 pesticides analyzed were detected in samples from all 9 wells in February 1998. The difference in the number of compounds detected in August and February could be related to the timing of the sampling relative to the irrigation season. The August sampling occurred at the end of the irrigation season, and all the compounds applied during that season could have been transported to the ground water. By February, several months had passed since the last irrigation; therefore, the compounds detected at that time were those that are persistent in the subsurface. 


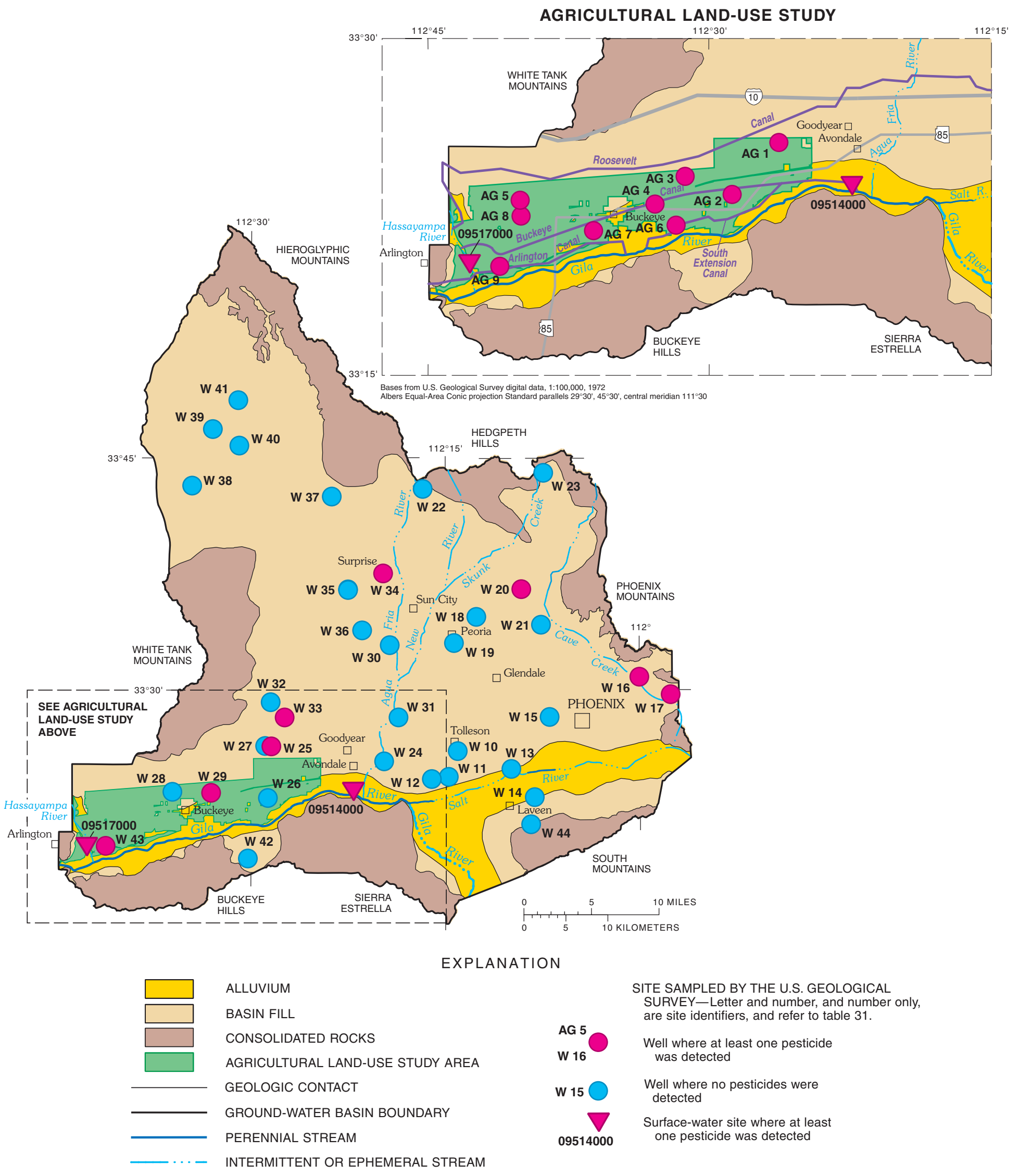

Figure 7. Locations of ground-water and surface-water sites where samples were collected for pesticide analyses, West Salt River Valley, Central Arizona Basins study area. 
Atrazine and deethylatrazine were the most frequently detected compounds and were detected in eight (89 percent) of the wells during both the August and February sampling periods. The ratio between deethylatrazine and atrazine can provide information about the degradation of atrazine in the subsurface. The ratio of deethylatrazine to atrazine was higher in samples collected in February 1998 than in the samples collected in August 1997 (fig. 8). These results indicate that atrazine is degrading in the shallow ground water between irrigation seasons (Edmonds and Gellenbeck, 2002). Bias due to matrix effects may affect the concentration of deethylatrazine; however, the difference in the ratios probably is not affected. Deisopropyl atrazine, which also is a pesticidedegradation product of atrazine was detected in wells AG6 and AG2 in August 1997 and in AG6 in February 1998 (E.M. Thurman, chemist, USGS, written commun., 1998). These data indicate that atrazine is breaking down in the ground water in this agricultural area.
For some pesticides, only the pesticide-degradation products were detected. One pesticide-degradation product-3,4-dichloroaniline-was detected in three samples collected in August 1997 and two samples collected in February 1998 (E.M. Thurman, chemist, USGS, written commun., 1998). The source of this degradation compound could be either propanil or linuron. Both propanil and linuron are used as herbicides. Propanil is used as a post-emergent herbicide on wheat and other crops. Linuron is used as a pre- and post-emergent herbicide on cotton and other crops. These compounds were not reported as being used in the agricultural LUS area (table 2), but nonreported uses could be sources of this degradation product. The presence of this pesticide-degradation compound in the absence of the parent compound is an indication of how quickly some pesticides breakdown in the environment.

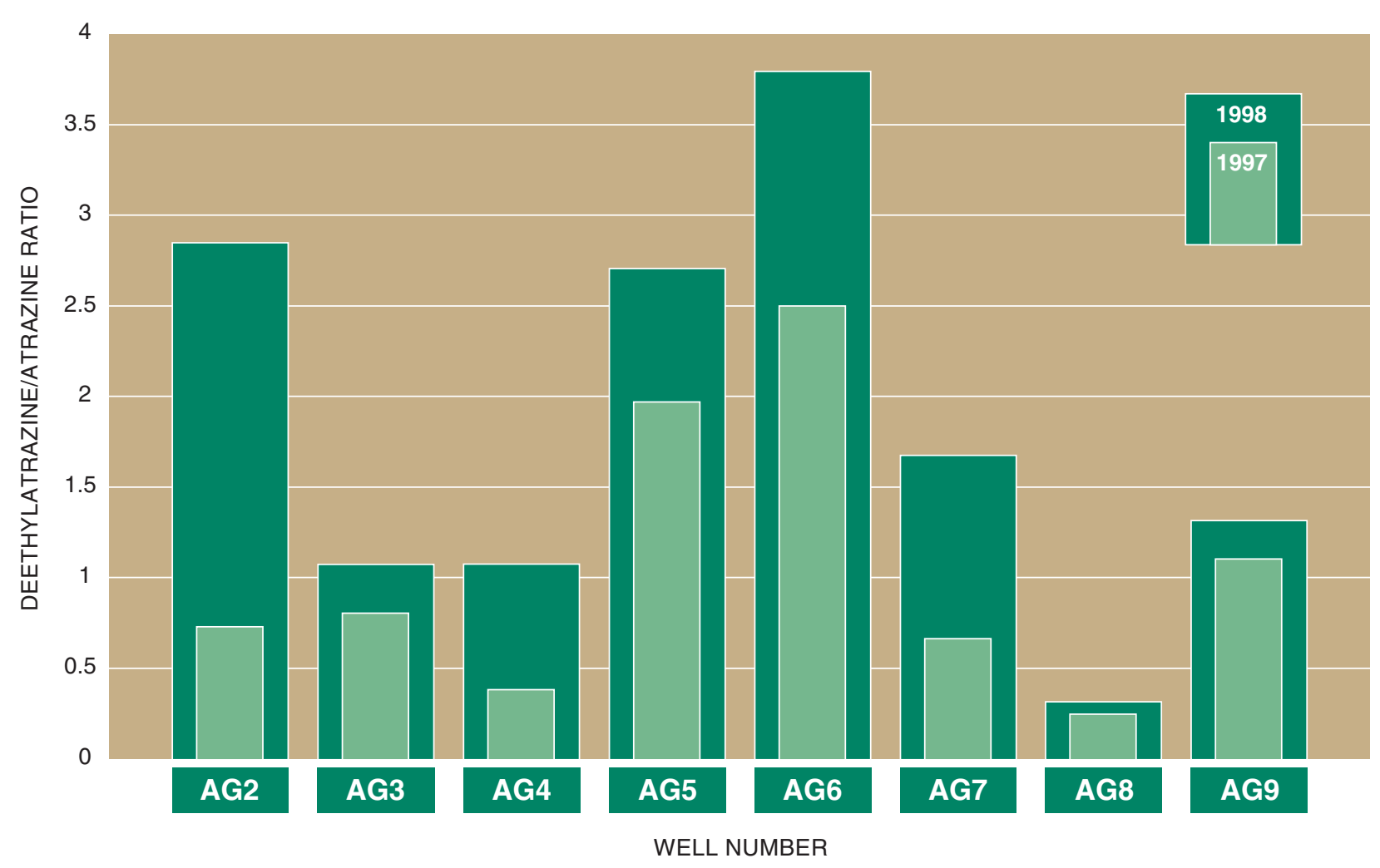

Figure 8. Deethylatrazine/atrazine ratios for samples from wells in the agricultural land-use study area, Central Arizona Basins study area, August 1997 and February 1998. 
Diuron was detected in samples collected from 5 wells in August 1997 (55 percent) and in samples collected from 4 wells in February 1998 (44 percent). Diuron is a herbicide that controls a wide variety of annual and perennial broadleaf and grassy weeds and is applied in agricultural and nonagricultural areas (Extension Toxicology Network, 1996). Diuron has been recommended for cotton crops in Arizona since 1955 (Brew and Baker, 1987; tables 1 and 2, this report). Although detections of diuron have been associated with urban land use (U.S. Geological Survey, 1999), diuron has been used for a long period of time in the West Salt River Valley on cotton crops, and its presence in ground water probably is related to land-surface application.

Simazine was detected in samples collected from 4 wells in August 1997 (44 percent) and in samples collected from 7 wells in February 1998 (78 percent). Prometon was detected in samples collected from 2 wells in August 1997 (22 percent) and in samples collected from 5 wells in February 1998 (55 percent). These detections indicate that pesticides applied for agricultural and nonagricultural uses are reaching the shallow ground water in this agricultural area.

DDE was detected in samples collected from 6 wells in August 1997 (67 percent) and in samples collected from 4 wells in February 1998 (44 percent). All but one of these detections were below the MRL (Tadayon and others, 1999). DDE is a degradation compound of DDT, an insecticide that was used in agricultural areas of the West Salt River Valley from 1944 until its use was banned in Arizona in 1965 (Brew and Baker, 1987). Previous studies have detected DDE in soils in the West Salt River Valley (Brown, 1993). DDT and DDE are highly persistent in the soil and have low solubilities in water; consequently, over long periods of time, it may be possible that the compounds leach into the ground water (Extension Toxicology Network, 1996).

Dieldrin has not been used for the last 10 years, but was detected in samples collected from 2 wells (AG5 and AG8) during August 1997 and February 1998. Dieldrin was recommended for cotton crops around 1955 when cotton was the primary crop in the area (Brew and Baker, 1987; table 1, this report). Dieldrin is an insecticide and also a pesticide-degradation compound of aldrin. Uses of aldrin and dieldrin were banned in 1972 except for subsurface termite control, nonfood agricultural uses, and moth proofing in closed systems in manufacturing processes (U.S. Environmental Protection Agency, 1980). By 1987, industry had removed these compounds from the marketplace for all uses (U.S. Environmental Protection Agency, 1995). The persistence of this insecticide is indicated by detections of dieldrin in shallow ground water more than 20 years after its use was banned.

\section{Surface Water}

Two surface-water sampling sites-Gila River at Buckeye Canal and Hassayampa River near Arlington-were used to determine the occurrence and distribution of pesticides in surface water affected by land-use activities in the West Salt River Valley (table 11). Although the sampling site on the Hassayampa River is outside the western boundary of the West Salt River Valley, it is included in this section because the base flow at this site is maintained by flow from the Buckeye Canal.

Twenty-three of the 86 pesticides analyzed were detected in 31 samples collected at the 3 sites used to represent the Gila River at Buckeye Canal (table 11) from February 1996 through April 1998. Of the 31 samples, 1 sample was collected during high-flow conditions due to releases from the reservoir upstream and 30 samples were collected during low-flow conditions (fig. 9). Concentrations of DDE in 12 samples (39 percent) exceeded the surface-water standards for agricultural irrigation, agricultural livestock watering, and fish consumption (State of Arizona, 1996; table 9, this report). Concentrations of dieldrin in 1 sample exceeded the fish-consumption and aquatic and wildlife standards for dieldrin. Eleven different pesticides were detected in more than 5 samples. Included in these 11 pesticides were 4 pre-emergent herbicides-simazine, trifluralin, EPTC, and dacthal - that were detected in samples collected during planting of crops, primarily between December and April (fig. 9).

Four other compounds detected in more than five samples were chlorpyrifos, diazinon, prometon, and malathion. Chlorpyrifos, diazinon, and malathion are insecticides used in agricultural and nonagricultural settings (Extension Toxicology Network, 1996).

A possible source of these four compounds is the same as the primary source of the water-outflow from the 91st Avenue WWTP. Diazinon and malathion are commonly used in residential applications, so it is 

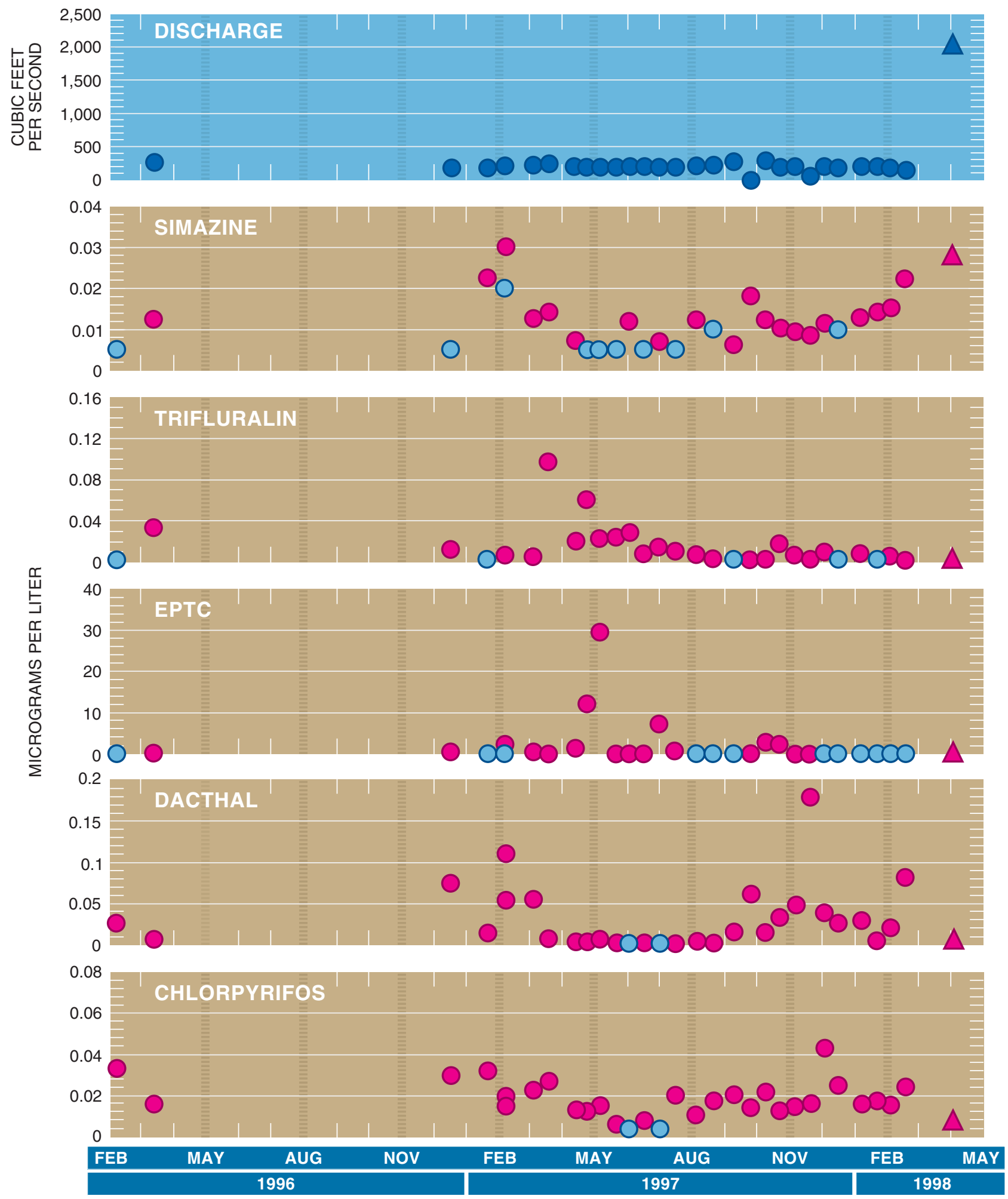

EXPLANATION

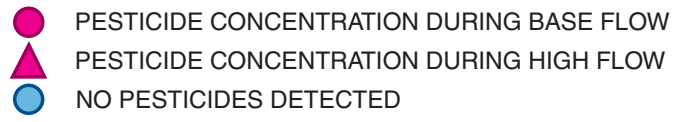

DISChARGE AT BASE FLOW

$\triangle$ DISCHARGE AT HIGH FLOW

Figure 9. Selected pesticides detected in surface-water samples collected from the Gila River at Buckeye Canal, West Salt River Valley, Central Arizona Basins study area, 1996-98. 

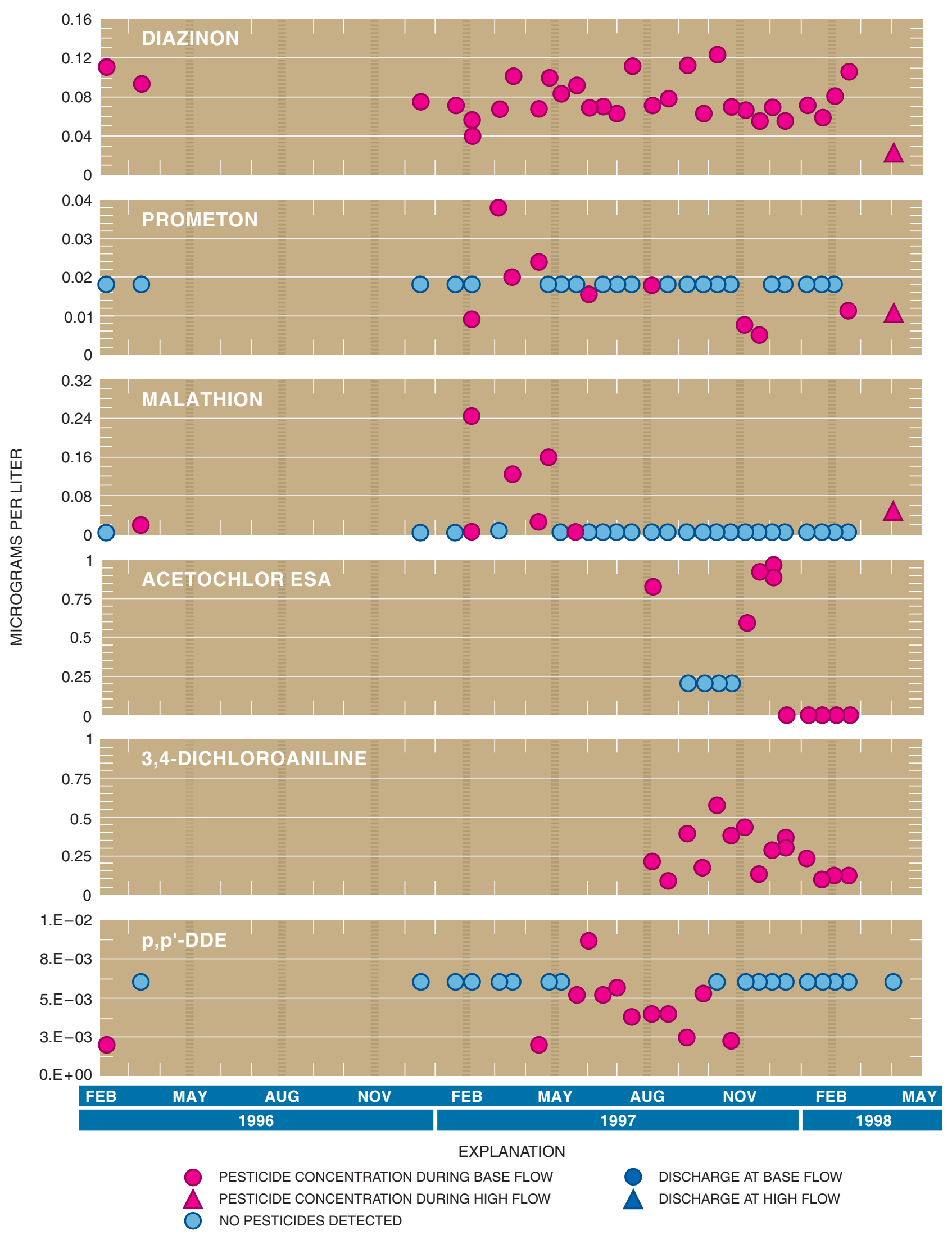

Figure 9. Continued. 
possible that these compounds are transported to the WWTP from household wastes and through the treatment processes to the outflow. The outflow is then diverted to the Buckeye Canal for use as irrigation. Chlorpyrifos and diazinon also may be from agricultural land downstream from the 91st Avenue WWTP. After application, transport of chlorpyrifos to the surface water may be delayed because it can be strongly adsorbed to soils (U.S. Department of Agriculture, 1995). Desorption from the soils can explain its persistence in surface water throughout the year. Diazinon has a low persistence in soils and seldom migrates below the top half inch in soil (Extension Toxicology Network, 1996). Malathion was detected in a few samples collected from February through May 1997. This period corresponds with the early planting season; therefore, the source of this insecticide may be primarily agricultural.

Pesticide-degradation compounds including DDE, acetochlor ESA, and 3,4-dichloroaniline were detected in several samples from Gila River at Buckeye Canal (Tadayon and others, 1998 and 1999; E.M. Thurman, chemist, USGS, written commun., 1998). The source of DDE probably is DDT that was applied in the past and is now present as DDE in the soils in the local area (Brown, 1993). The parent compounds of acetochlor ESA and 3,4-dichloroaniline were not detected in the surface-water samples.

The detections of only pesticide-degradation products for a short period during the irrigation season indicates that the pesticides are degrading quickly in the environment. Detections of 3,4-dichloroaniline were more numerous than detections of other degradation compounds probably because of the degradation of several different pesticides including diuron (tables $\mathbf{1}$ and 2).

The sample collected in April 1998 from the Gila River at Buckeye Canal (09514100) is from a release of surface water from a reservoir upstream on the Salt River and included detectable concentrations of some pesticide compounds (fig. 9). Of the 11 compounds most often detected in samples from this site, 4 compounds from agricultural and nonagricultural uses were detected in this sample (fig. 9). Of the 4 compounds detected, only simazine had a higher concentration in this sample than in samples collected during low-flow conditions. Lower concentrations may be the result of dilution. The source-and-transport processes associated with the high concentration of simazine in this sample are unknown.

Twenty-six of the 86 pesticides analyzed were detected in 38 samples collected from the Hassayampa River near Arlington (table 11) between February 1996 and February 1998. Six samples were collected during high-flow conditions, and 31 samples were collected during low-flow conditions. Thirty-three samples (89 percent) exceeded the agricultural irrigation, agricultural livestock watering, and fish-consumption standards for DDE (State of Arizona, 1996; table 9, this report). Fourteen different pesticides were detected in more than 5 samples (fig. 10). Of these 14 pesticides, 6 pre-emergent herbicides - simazine, trifluralin, EPTC, dacthal, linuron, and pendimethalin-were detected between October and May. Most of these compounds are applied to the fields before the appearance of broadleaf weeds and grasses; linuron and pendimethalin also may be applied after grasses and weeds have sprouted.

The other 8 pesticides that were detected in more than 5 samples were chlorpyrifos, diazinon, prometon, malathion, atrazine, deethylatrazine, DDE, and 3,4-dichloroaniline. Chlorpyrifos and diazinon were detected in surface-water samples from this site throughout the year and are similar to detections at the Gila River at Buckeye Canal. The highest concentrations of chlorpyrifos and diazinon in the Hassayampa River near Arlington (0.154 and $0.207 \mu \mathrm{g} / \mathrm{L}$, respectively) are higher than those from the Gila River at Buckeye Canal (0.0429 and $0.123 \mu \mathrm{g} / \mathrm{L}$, respectively). Higher concentrations in the Hassayampa River probably are due to the denser agricultural land use upstream from this site compared with the Gila River at Buckeye Canal. Variation in replicate samples could be related to sample processing and analytical procedures. Because these compounds are used in agricultural and nonagricultural settings, the source of the compounds could be outflow from the 91st Avenue WWTP, runoff from agricultural fields downstream from the 91st Avenue WWTP, and (or) desorption from soils.

Deethylatrazine, DDE, and 3,4-dichloroaniline are degradation compounds of pesticides used in the area (atrazine, DDT, and diuron, respectively). Deethylatrazine was detected during April-December- the same time period that the parent compound, atrazine, was detected. Detections of DDE in samples from the Hassayampa River near Arlington occurred throughout the year. The DDE may be transported in runoff from agricultural fields (Brown, 1993) upgradient from this site. Detections of 3,4-dichloroaniline were expected because diuron, one of several parent compounds for this degradation product, was used in the area (tables 1 and 2). 

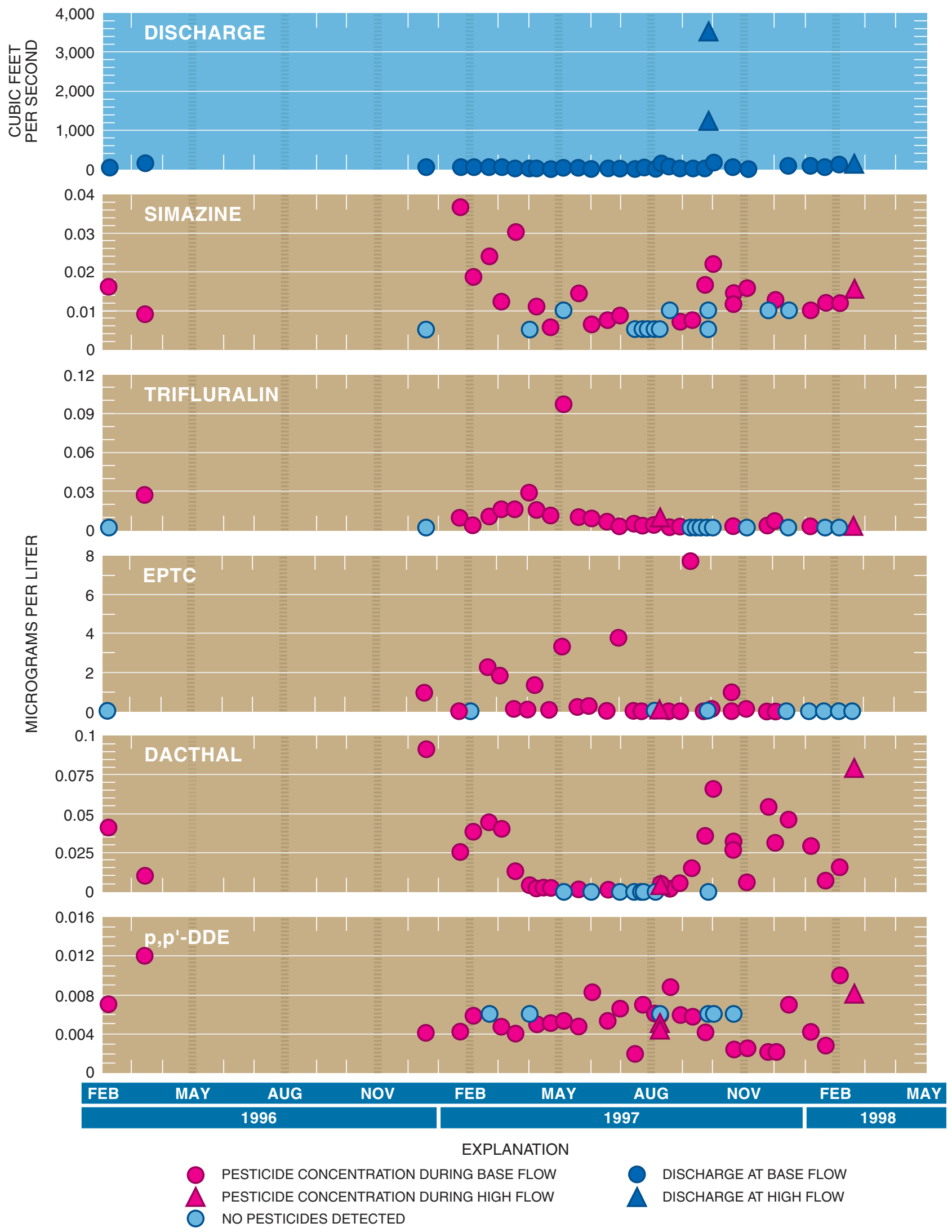

Figure 10. Selected pesticides detected in surface-water samples collected from the Hassayampa River near Arlington, West Salt River Valley, Central Arizona Basins study area, 1996-98. 

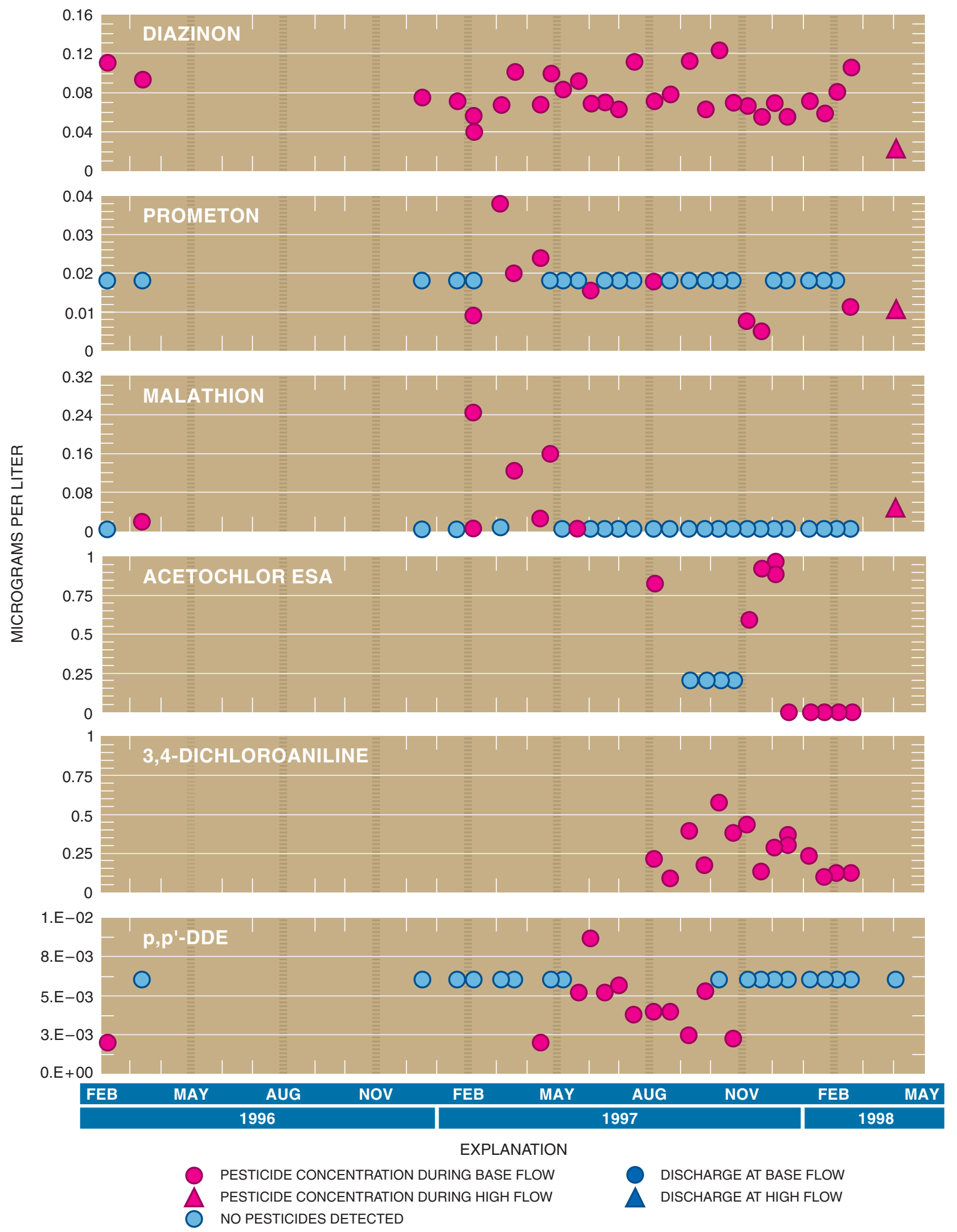

Figure 10. Continued. 


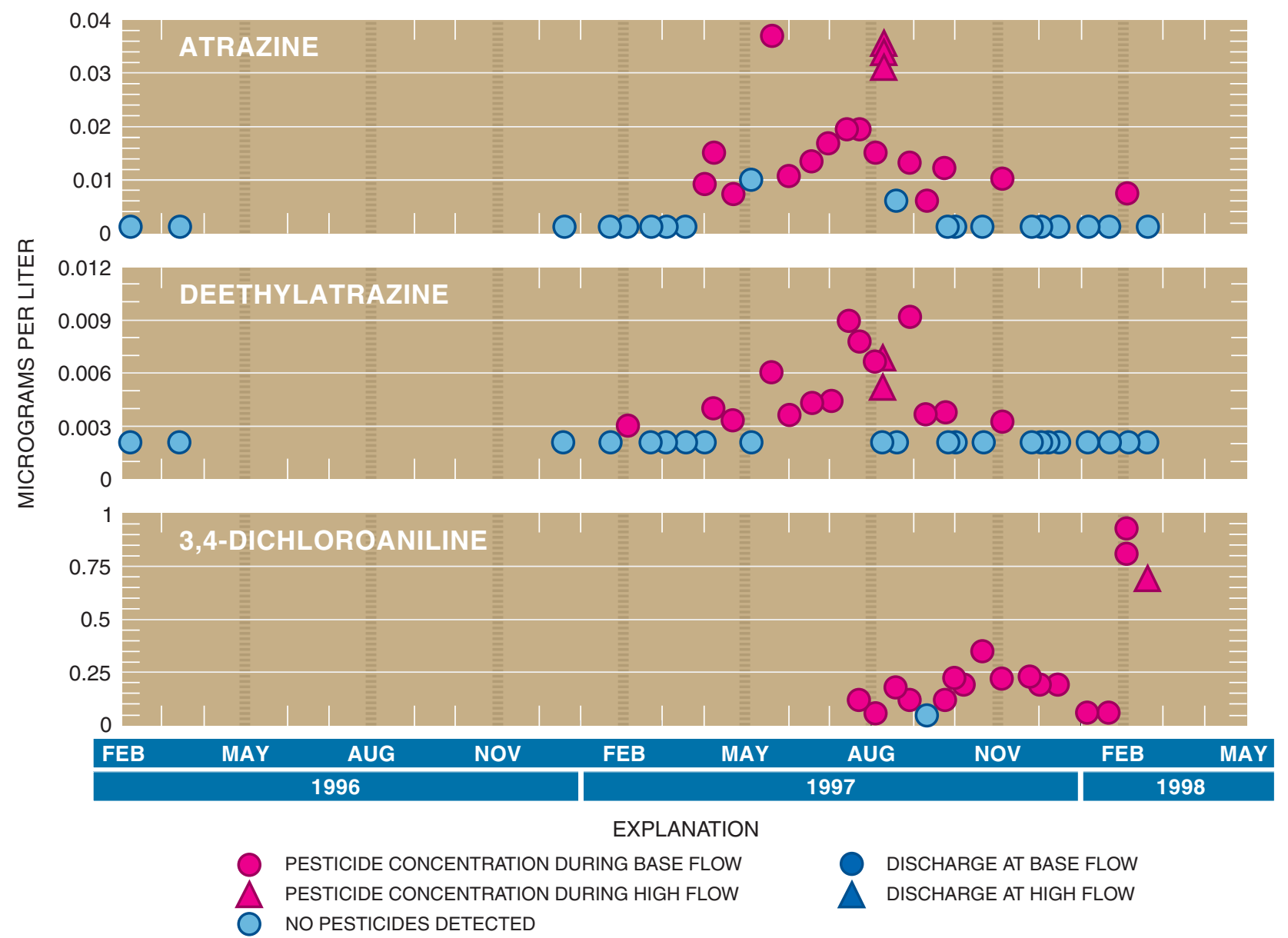

Figure 10. Continued.

Six high-flow samples were collected from the Hassayampa River near Arlington in August 1997 (three samples), September 1997 (two samples), and February 1998 (one sample). In the high-flow sample collected in September 1997, pesticide concentrations were low or nondetected. High-flow samples collected in August 1997 had detections of 13 compounds. Comparison between concentration in high-flow and low-flow samples indicates that sometimes concentrations were higher in high-flow samples for some pesticides and at other times concentrations of these same pesticides were equal to or lower than those in low-flow samples. Concentrations of prometon, cyanazine, atrazine, methyl parathion, and carbaryl were higher in high-flow samples. Concentrations of deethylatrazine, DDE, chlorpyrifos, trifluralin, EPTC, dacthal, and pendimethalin in the high-flow samples were similar to concentrations in low-flow samples. Concentrations of dacthal, DDE, prometon, and 3,4-dichloroaniline were somewhat higher in the highflow sample collected in February 1998, than in other samples collected earlier in 1998 at lower discharges. Variation in concentrations due to processing and analytical techniques could account for some of the differences in the concentrations of DDE and prometon. Reasons for the differences in concentrations between high-flow and low-flow samples could include differences in time of application, degree of adsorption of the compounds to soil, and origin of runoff. Without more detailed information, the cause of these differences cannot be determined.

Concentrations of some pesticide compounds in surface water in the West Salt River Valley appear to vary throughout the year, and concentrations of other pesticide compounds remain somewhat constant. Preemergent pesticides, which include simazine, trifluralin, EPTC, and dacthal, exhibit the most pronounced seasonal variations (figs. 9 and 10). At the Gila River at Buckeye Canal, these compounds were detected primarily from December through April; at the Hassayampa River near Arlington, these compounds were detected primarily from October through May. 
The Hassayampa River drainage is larger than the Gila River at Buckeye Canal drainage; therefore, travel times for compounds can be longer, which results in longer detection periods.

DDE was detected in samples collected from the Gila River at Buckeye Canal from April through December 1997 and in samples collected from the Hassayampa River near Arlington throughout the sampling period. Because this compound was detected at the Gila River at Buckeye Canal during the intense irrigation season, it is possible that the source of this compound is the agricultural fields upstream and not outflow from the 91st Avenue WWTP, which is the source of most of the water at this site. Detections of DDE at the Hassayampa River near Arlington throughout the sampling period indicate that the transport mechanism operates all year. For those compounds that occur throughout the year, likely sources are WWTP outflow, desorption from soils, and runoff from agricultural areas.

One major source of water for the Gila River at Buckeye Canal and Hassayampa River near Arlington is outflow from the 91st Avenue WWTP, which is available throughout the year and is primarily affected by urban activities. Although the reuse of water is important for conservation efforts, it provides a mechanism for pesticides in the water to move from urban land-use areas to agricultural land-use areas. The reuse of treated effluent for irrigation water and the reuse of irrigation tail water result in a complex mixture of pesticides in the West Salt River Valley. This complex mixture and the water-management practices in the West Salt River Valley make it difficult to determine the original sources of pesticides in surface water and ground water. Although the presence of some pesticides clearly is the result of historical use for agriculture, the presence of others could be related to either agriculture or urban land use. The presence of DDE in shallow ground water and surface water is the result of agricultural use of DDT in this area more than 30 years ago. The presence of some herbicides including simazine and prometon can be related to agricultural or urban land use. Insecticides that are linked to urban uses, including chlorpyrifos, diazinon, and malathion, also were detected in the Gila River at Buckeye Canal. Without more detailed studies of water sources, it is impossible to identify which land-use activity is the source of some pesticides in the West Salt River Valley.

\section{Volatile Organic Compounds}

Ground water and surface water sampled in the CAZB during 1996-98 contained a large variety of VOCs, especially in those areas where urban land use is prevalent. Most of the detectable concentrations were low and less than water-quality limits. VOCs are present in ground water and surface water in some areas of the CAZB, but not at a level that requires action by an enforcement agency. Identification of specific sources of VOCs is difficult because of the various pathways that ground water may have taken to the well that was sampled or the various sources of water contributing to flow at the surface-water sampling location. The difficulty of identifying specific sources was increased because the detectable concentrations of VOCs were very low. Generallyknown sources of VOCs are included in this report as a guide for the reader. Specific sources of VOCs in a localized area are presented if they were pertinent, but this study did not verify that those sources contributed the VOCs to the well or surface-water sampled. The cooccurrence of several VOCs or VOCs with pesticides and nitrate is not considered in the current implementation of water-quality limits. Many samples from ground water and surface water in the CAZB that had detectable concentrations of VOCs and (or) pesticides also had detectable concentrations of more than one compound. Little is known about the potential health effects from the co-occurrence of several VOC and pesticide compounds (Squillace and others, 1999).

\section{Minimally Developed Basin}

In the Sierra Vista subbasin, ground-water samples were collected to evaluate the occurrence and distribution of VOCs. No surface-water samples were collected for VOC analyses in this subbasin. Eleven of the 87 VOCs analyzed were detected in 14 wells (74 percent; fig. 11 and table 12). 1,2,4-trimethylbenzene was the most frequently detected compound and was detected in 10 samples. PCE was detected in 3 samples; chloromethane, dichlorodifluoromethane, and carbon disulfide were detected in 2 samples; and bromodichloromethane, tribromomethane, benzene, chlorobenzene, acetone, and tetrahydrofuran were detected in 1 sample each. Two detections of trichloromethane are not considered in this report because detections occurred in a field blank in the same time period. Concentrations were not higher than water-quality limits established by the USEPA (1996) and the State of Arizona (1996; table 13, this report). 


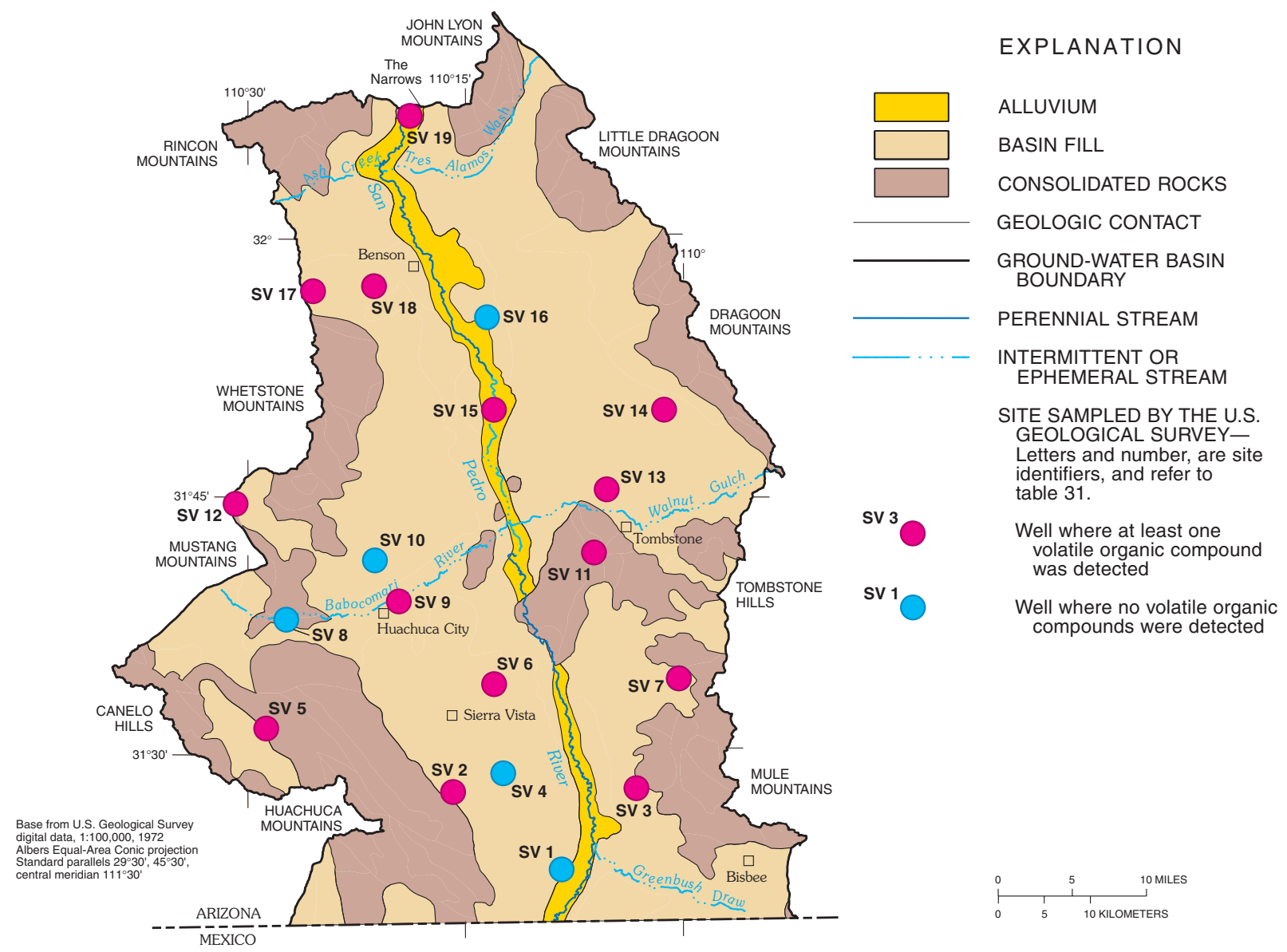

Figure 11. Locations of ground-water sites where samples were collected for volatile organic compound analyses, Sierra Vista subbasin, Central Arizona Basins study area, 1996-97.

Table 12. Number of volatile organic compounds detected in ground-water samples in the Sierra Vista subbasin, Central Arizona Basins study area, 1996-97

Volatile organic compounds

\begin{tabular}{lcccc}
\cline { 2 - 4 } Ground-water study name & $\begin{array}{c}\text { Samples } \\
\text { collected }\end{array}$ & Detections & $\begin{array}{c}\text { Samples with } \\
\text { detectable } \\
\text { concentrations }\end{array}$ & $\begin{array}{c}\text { Compounds } \\
\text { detected }\end{array}$ \\
\hline Vista subbasin sub-unit survey & 19 & 25 & 14 & 11 \\
\hline
\end{tabular}


Table 13. Maximum Contaminant Levels, human health advisory limits, aquifer water-quality standards, and surface-water standards for volatile organic compounds detected in ground-water and surface-water samples, Central Arizona Basins study area, 1996-98

[Constituents are dissolved and are reported in micrograms per liter; HAL, U.S. Environmental Protection Agency established human health advisory level for drinking water; MCL, Maximum Contaminant Level; E, concentration is estimated; dashes indicate no data]

\begin{tabular}{|c|c|c|c|c|c|c|c|c|c|}
\hline \multirow[b]{3}{*}{ Constituent } & \multicolumn{2}{|c|}{$\begin{array}{l}\text { Concentration range } \\
\text { of detections }\end{array}$} & \multicolumn{2}{|c|}{$\begin{array}{l}\text { U.S. Environmental } \\
\text { Protection } \\
\text { Agency }\end{array}$} & \multicolumn{5}{|c|}{ State of Arizona ${ }^{2}$} \\
\hline & \multirow[b]{2}{*}{ Ground water } & \multirow[b]{2}{*}{ Surface water } & \multirow[b]{2}{*}{$\mathrm{HAL}^{3}$} & \multirow[b]{2}{*}{$\begin{array}{c}\text { Primary } \\
\text { MCL }\end{array}$} & \multicolumn{5}{|c|}{ Surface water } \\
\hline & & & & & $\begin{array}{l}\text { Aquifer } \\
\text { water- } \\
\text { quality } \\
\text { standard }\end{array}$ & $\begin{array}{l}\text { Fish } \\
\text { consump- } \\
\text { tion }\end{array}$ & $\begin{array}{c}\text { Agri- } \\
\text { cultural } \\
\text { livestock } \\
\text { watering }\end{array}$ & $\begin{array}{c}\text { Agri- } \\
\text { cultural } \\
\text { irrigation }\end{array}$ & $\begin{array}{c}\text { Aquatic } \\
\text { and } \\
\text { wildlife }\end{array}$ \\
\hline Benzene & E0.006-E.020 & E.015 & --- & 5 & 5 & 120 & --- & --- & 700 \\
\hline Bromodichloromethane & E.01-297 & E0.1-4 & --- & $5_{100}$ & --- & 22 & --- & --- & --- \\
\hline Tetrachloromethane & --- & E.06 & --- & 5 & 5 & 5.5 & --- & --- & 1,100 \\
\hline Chloromethane & E.008-E.1 & E.04-E.218 & 3 & --- & --- & --- & --- & --- & 15,000 \\
\hline 1,1,1-Trichloroethane & E.01 & E.01 & --- & 200 & 200 & --- & --- & --- & 1,600 \\
\hline 1,1-Dichloroethene & E.03 & --- & --- & 7 & 7 & 4.5 & --- & --- & 950 \\
\hline Methyl tert-butyl ether & E.05-E.06 & E.04-E.061 & ${ }^{6} 20-200$ & --- & --- & --- & --- & --- & --- \\
\hline Tetrachloroethene & Е.005-5.48 & E.03-3.6 & --- & 5 & 5 & 11 & --- & --- & 680 \\
\hline Trichloroethene & E.01-1.26 & Е.02-E.04 & --- & 5 & 5 & --- & --- & --- & 1,300 \\
\hline Trichloromethane & $.008-.498$ & $.75-8.1$ & --- & ${ }^{5} 100$ & --- & --- & --- & --- & --- \\
\hline
\end{tabular}

VOCs were detected in ground-water samples from areas of the Sierra Vista subbasin that have urban land use and in areas that have rangeland. Although this subbasin is characterized as having minimal urban development (Gellenbeck and Coes, 1999), detections of VOCs indicate that ground water in localized areas could be affected by human activities. Detections of VOCs per well (fig. 12) for each ground-water study also indicate that the distribution corresponds with the amount of urban land use in each ground-water basin. Detections of VOCs per well were determined by dividing the number of VOC detections by the number of samples with detections. The highest number of detections of VOCs per well are in basins with the largest amount of urban-land use. The Upper Santa Cruz Basin SUS, the West Salt River Valley SUS, and the LUS each had more than two detections of VOCs per well (fig. 12), and the Sierra Vista SUS had fewer than two detections of VOCs per well. Statistical comparisons of VOC results from the SUSs identified no difference among the SUSs.
The most frequently detected VOC, 1,2,4-trimethylbenzene, has several possible sources. The largest users of 1,2,4-trimethylbenzene are chemical companies that make trimellitic anhydride. Dyes and perfumes also are sources of this compound (Sax and Lewis, 1987). Specific sources in the Sierra Vista subbasin could not be identified.

A sample from well SV18 contained the most VOCs (three)—chloromethane, carbon disulfide, and 1,2,4-trimethylbenzene. SV18 is south of Interstate 10 and downgradient from a gas station, which could be sources for the VOCs detected. Natural sources of chloromethane from fungus (Harper, 1985), forest fires (Palmer, 1976), and volcanic gases (Inn and others, 1981), and natural sources of carbon disulfide from volcanic gases (Inn and others, 1981) and mushrooms (Turner and others, 1975) also could have contributed the VOCs to the ground water. 


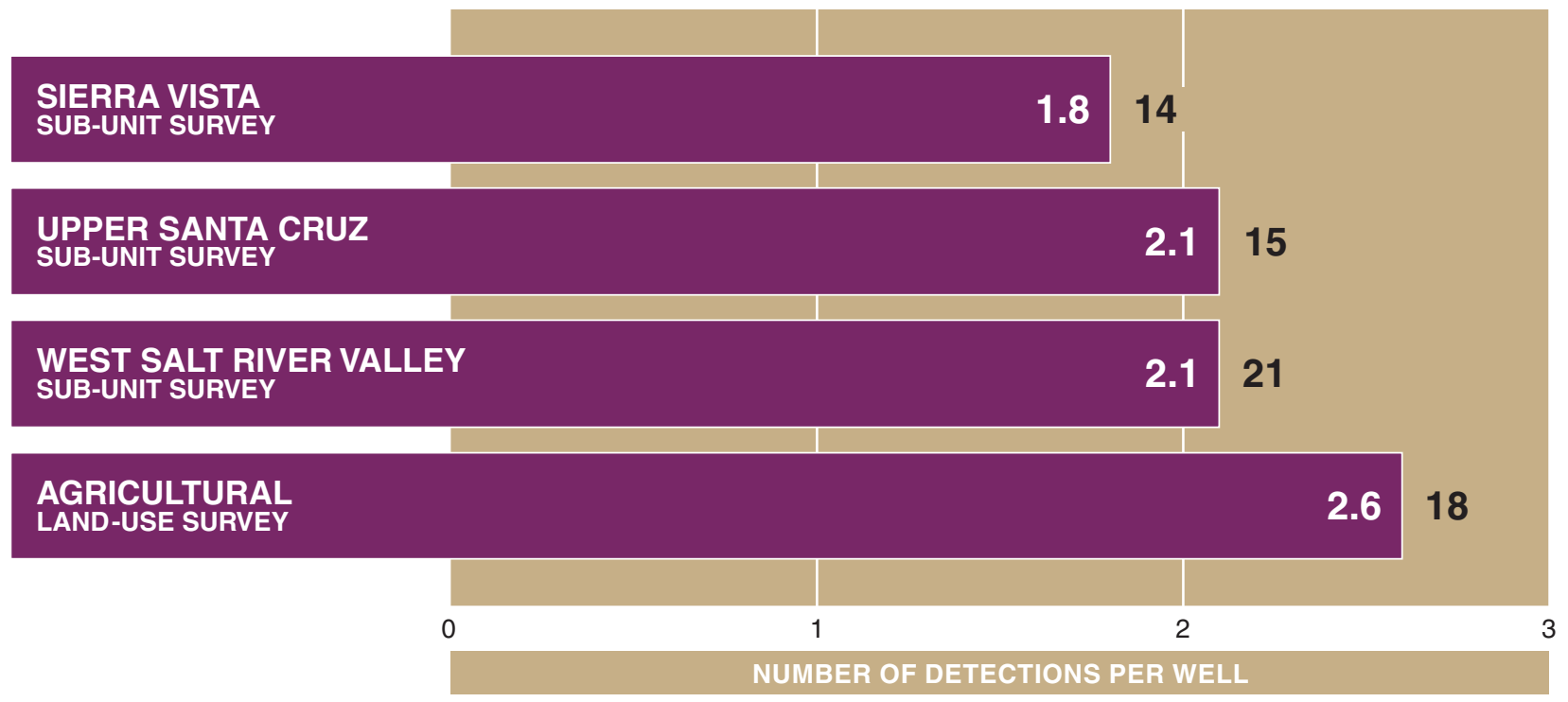

Figure 12. Number of volatile organic compound detections per well, Central Arizona Basins study area, 1996-98. Detections per well equal number of volatile organic compound detections divided by number of samples with detections. Numbers in black are the number of samples with volatile organic compounds detected.

Samples from two wells-SV2 and SV9-contained two VOCs, one of which was PCE. PCE is a solvent and one of the most commonly detected VOCs in ground water in the United States (Squillace and others, 1999). SV2 is in a housing area where PCE is not likely used. SV9 is downgradient from a landfill that could be the source of PCE and dichlorodifluoromethane that also was detected in the sample from this well. The Babocomari River, which is a few hundred feet from SV9, may aid in the transport of compounds to ground water that flows to this well.

\section{Developed Basins}

Detections of VOCs in the West Salt River Valley and the Upper Santa Cruz Basin unexpectedly are similar to the results for ground water in the Sierra Vista subbasin. The presence of VOCs generally reflects the urban land uses in these basins. The largest number of detections occurred in ground-water and surface-water samples from the West Salt River Valley.

\section{Upper Santa Cruz Basin}

Detections of VOCs in the Upper Santa Cruz Basin indicate that activities in urban areas in this basin are affecting the ground-water and surface-water quality.
Detections of VOCs in ground water and surface water in the Nogales area support findings of previous studies conducted in this area (Marsh, 1994). Detections of VOCs in ground water near Tucson indicate that water quality is being affected by VOCs used on the land surface.

Eleven VOCs (of the analytical suite of 86) were detected in samples from 15 wells (52 percent) in the Upper Santa Cruz Basin (table 14; fig. 13). The most commonly detected VOC was trichloromethane, which was detected in seven samples; chloromethane and 1,4-dichlorobenzene were detected in five samples; PCE was detected in four samples; methylbenzene was detected in three samples; bromodichloromethane and 1,2-dichlorobenzene were detected in two samples; and trichlorofluoromethane, dichloro-difluoromethane, trichloroethene, and 1,2,4-trimethylbenzene were detected in one sample each. Twenty-four detections of 1,2,4-trimethylbenzene were the result of sample contamination; the compound was detected in field blanks during the same time period that it was detected in environmental samples. The concentration of PCE in one sample exceeded established limits of the USEPA (1996) and the State of Arizona (1996). 
Table 14. Number of volatile organic compounds detected in ground-water and surface-water samples, Upper Santa Cruz Basin, Central Arizona Basins study area, 1996-98

\begin{tabular}{|c|c|c|c|c|c|c|}
\hline \multirow[b]{2}{*}{$\begin{array}{l}\text { Ground-water study name or } \\
\text { surface-water site name }\end{array}$} & \multirow[b]{2}{*}{$\begin{array}{l}\text { Medium } \\
\text { sampled }\end{array}$} & \multirow[b]{2}{*}{$\begin{array}{l}\text { Surface-water } \\
\text { site number }\end{array}$} & \multicolumn{4}{|c|}{ Volatile organic compounds } \\
\hline & & & $\begin{array}{l}\text { Samples } \\
\text { collected }\end{array}$ & Detections & $\begin{array}{l}\text { Samples } \\
\text { having } \\
\text { detectable } \\
\text { concentra- } \\
\text { tions }\end{array}$ & $\begin{array}{c}\text { Compounds } \\
\text { detected }\end{array}$ \\
\hline Upper Santa Cruz sub-unit survey & Ground water & --- & 29 & 32 & 15 & 11 \\
\hline Nogales Wash near the Nogales & Surface water & 312314110565601 & 2 & 7 & 2 & 5 \\
\hline \multicolumn{7}{|c|}{ Downstream from Nogales International Wastewater Treatment Plant } \\
\hline Santa Cruz River near Rio Rico, Arizona & Surface water & 312809110592801 & 2 & 6 & 2 & 4 \\
\hline Santa Cruz River at Santa Gertrudis Lane & Surface water & 313343110024701 & 2 & 3 & 1 & 3 \\
\hline Santa Cruz River at Tubac, Arizona & Surface water & 09481740 & 2 & 6 & 2 & 4 \\
\hline
\end{tabular}

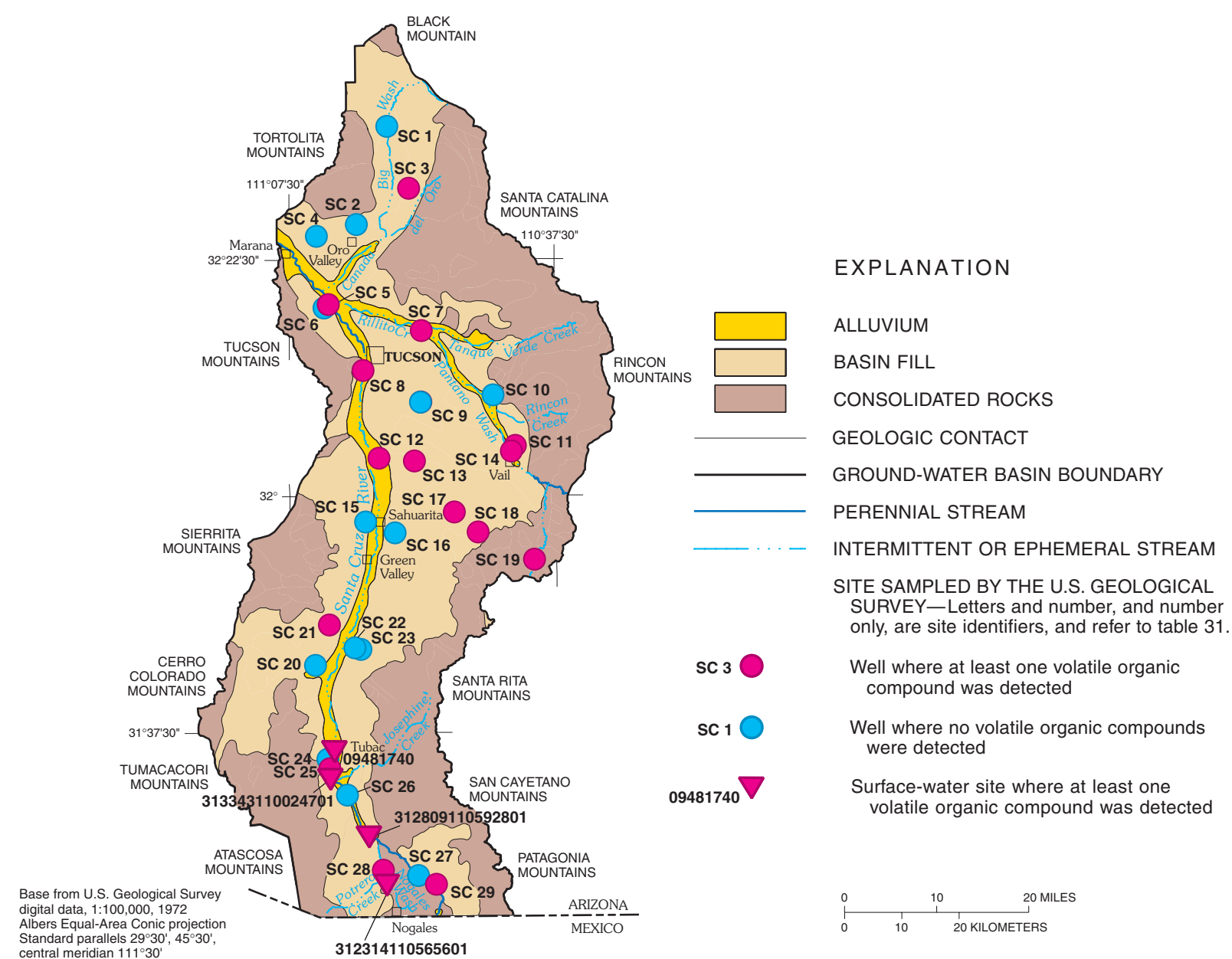

Figure 13. Locations of ground-water and surface-water sites where samples were collected for volatile organic compound analyses, Upper Santa Cruz River Basin, Central Arizona Basins study area. 
Samples from wells SC5 (five VOCs detected), SC17 (four VOCs detected), and SC28 (five VOCs detected) had the most VOCs detected. The following VOCs were detected in samples from at least two wells: bromodichloromethane (SC17 and SC28), 1,4-dichlorobenzene (SC5 and SC17), trichloromethane (SC5, SC17, and SC28), PCE (SC5 and SC28), and 1,2-dichlorobenzene (SC5 and SC28). In addition, a sample from well SC28 also contained detectable concentrations of TCE. SC28 is no longer used because of the high PCE concentrations (Pat Clymer, Arizona Department of Environmental Quality, oral commun., 1999); the concentration of PCE in this sample, $5.48 \mu \mathrm{g} / \mathrm{L}$, is above the aquifer water-quality standard of $5 \mu \mathrm{g} / \mathrm{L}$ (State of Arizona, 1996). According to Western Technologies, Inc. (1997), the source of PCE in this well is local to the area around the well. Well SC28 is at a produce shipping center near Nogales that is a high-traffic area for vehicles. Although the detection of PCE at well SC28 was the only detection of a VOC in ground water in this area, there is known ground-water contamination by VOCs, including TCE and many of its degradation products, in the Nogales area (Marsh, 1994). Well SC5 is downgradient from effluent releases in the Santa Cruz River and is about $1 \mathrm{mi}$ west of the river. A landfill near the river (Marsh, 1994) could be a source of the VOCs detected in ground water from this well. Well SC17 is in a newly developed residential area that was used originally for rangeland. There are no obvious sources of VOCs near this well.

Samples from seven wells-SC5, SC8, SC12, SC17, SC19, SC25, and SC28 - had detectable concentrations of trichloromethane. This compound is a solvent and a common byproduct of chlorinated drinking water, as well as a disinfection byproduct that may enter ground water through lawn irrigation, leaking water mains, and sewers (Squillace and others, 1999). Morrow (1999) linked trichloromethane in domestic wells to the use of bleach for disinfecting the wells; this practice also is used in Arizona (Doug Towne, hydrologist, Arizona Department of Environmental Quality, oral commun., 1999).

Detections of PCE also indicate that VOCs used at the land surface are reaching the ground water in this basin. PCE was detected in samples from wells SC5,
SC8, and SC28. Marsh (1994) lists many potential sources of PCE, as well as local use of solvents containing PCE.

Surface-water samples were collected during lowflow conditions at three sites along the Santa Cruz River and one site on Nogales Wash, which is tributary to the Santa Cruz River (table 8). Ten VOCs were detected in samples collected at all sites in November 1997 and June 1998 - chloroform, toluene, benzene, chloromethane, dichloromethane, 1,4-dichlorobenzene, carbon disulfide, styrene, 4-isopropyl-1-methylbenzene, and methyl tert-butyl ether (MTBE). Concentrations were low for these VOCs; however, the detections indicate that there is some effect from urban activities upstream from the sampling sites near Nogales, Arizona.

\section{West Salt River Valley}

Detections of VOCs in ground-water and surfacewater samples from the West Salt River Valley exceeded those for any other basin during 1996-98. Activities in the large urban area in this basin are the likely source of most of the VOCs. Several known ground-water contamination sites are in the West Salt River Valley (Marsh, 1994). Surface-water samples from the largest WWTP in the basin contained several VOCs, which probably derive from urban wastewater and the chemical reactions that occur during the wastewater-treatment processes. Surface-water samples from the Gila River at Buckeye Canal, downstream from the WWTP, contain fewer VOCs than samples from the WWTP. This difference could indicate that volatilization of compounds is occurring.

\section{Ground Water}

Eighteen VOCs were detected in 21 (70 percent) of the 30 wells sampled for VOCs in the West Salt River Valley SUS (fig. 14; table 15). Most samples collected for this SUS were analyzed for 86 VOCs; samples collected before March 27, 1996, were analyzed for 60 VOCs, and the MRLs for these analyses were higher than the MRLs for analyses of samples collected after March 27. None of the detections of VOCs occurred in the five samples collected before March 27, 1996. 
AGRICULTURAL LAND-USE STUDY

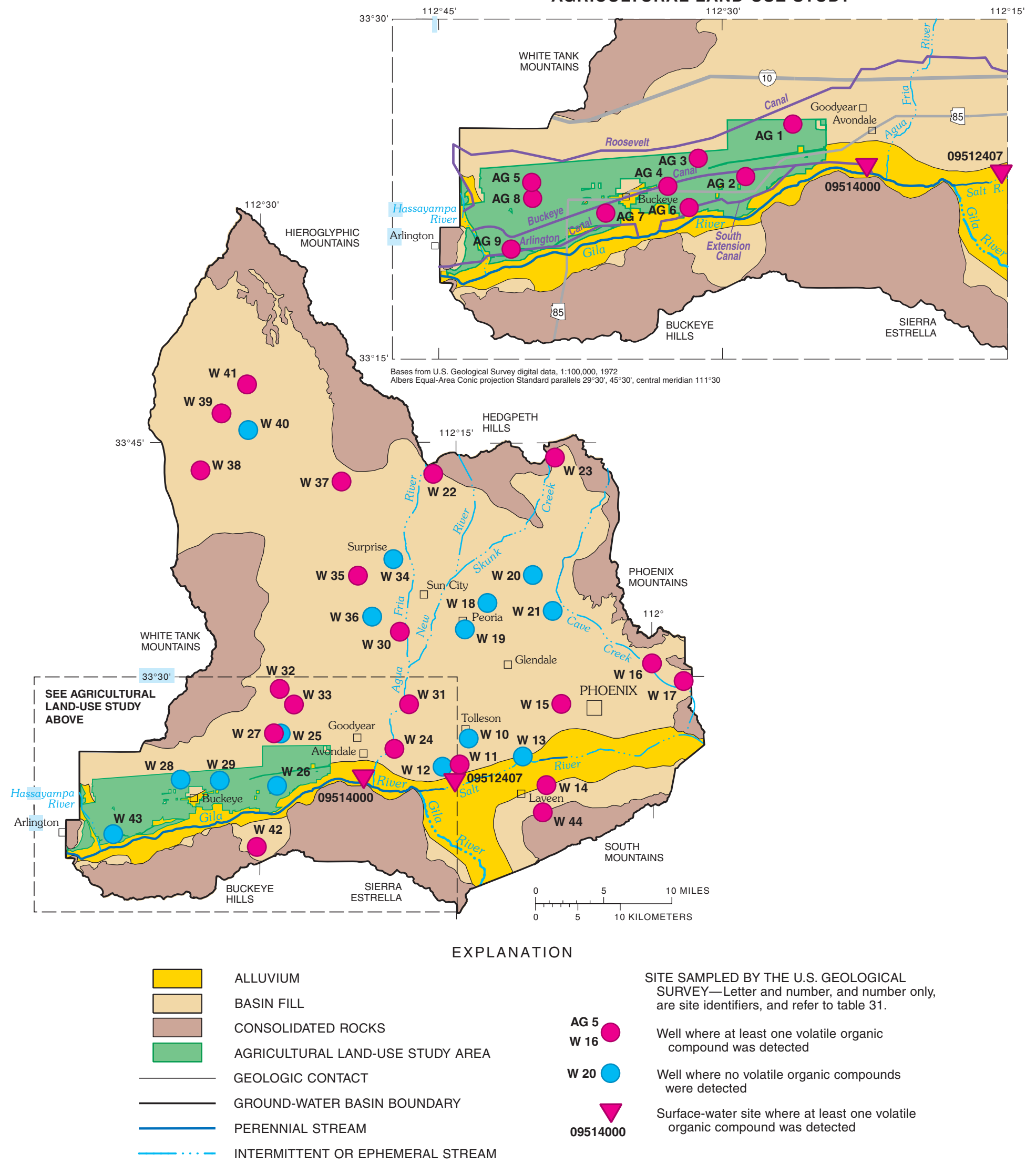

Figure 14. Locations of ground-water and surface-water sites where samples were collected for volatile organic compound analyses, West Salt River Valley, Central Arizona Basins study area. 
Table 15. Number of volatile organic compounds detected in ground-water and surface-water samples, West Salt River Valley, Central Arizona Basins study area, 1996-98

[Dashes indicate that the information is not applicable. Data for agricultural land-use study represents two sampling efforts—August 1997 and February 1998 ]

\begin{tabular}{|c|c|c|c|c|c|c|}
\hline \multirow[b]{2}{*}{$\begin{array}{l}\text { Ground-water study name or } \\
\text { Surface-water site name }\end{array}$} & \multirow[b]{2}{*}{$\begin{array}{l}\text { Medium } \\
\text { sampled }\end{array}$} & \multirow[b]{2}{*}{$\begin{array}{l}\text { Surface-water } \\
\text { site number }\end{array}$} & \multicolumn{4}{|c|}{$\begin{array}{l}\text { Volatile organic } \\
\text { compounds }\end{array}$} \\
\hline & & & $\begin{array}{l}\text { Samples } \\
\text { collected }\end{array}$ & Detections & $\begin{array}{l}\text { Samples with } \\
\text { detections }\end{array}$ & Compounds \\
\hline $\begin{array}{l}\text { West Salt River Valley } \\
\text { sub-unit survey }\end{array}$ & Ground water & --- & 30 & 44 & 21 & 18 \\
\hline Agricultural land-use study & $\begin{array}{l}\text { Shallow ground } \\
\text { water }\end{array}$ & --- & 18 & 46 & 18 & 10 \\
\hline $\begin{array}{l}\text { 91st Avenue Wastewater } \\
\text { Treatment Plant outfall }\end{array}$ & Surface water & 09512407 & 6 & 77 & 6 & 26 \\
\hline Gila River at Buckeye Canal & Surface water & & 5 & 34 & 5 & 15 \\
\hline $\begin{array}{l}\text { Gila River above diversions } \\
\text { at head of Buckeye Canal }\end{array}$ & & 09513990 & & & & \\
\hline $\begin{array}{l}\text { Buckeye Canal near } \\
\text { Avondale }\end{array}$ & & 09514000 & & & & \\
\hline $\begin{array}{l}\text { Gila River at Estrella } \\
\text { Parkway near Goodyear }\end{array}$ & & 09514100 & & & & \\
\hline
\end{tabular}

The most frequently detected VOC was 1,2,4trimethyl-benzene, which was detected in eight samples. Chloromethane, carbon disulfide, and iodomethane were detected in four samples. Trichloromethane, TCE, and 1,4-dichlorobenzene were detected in three samples. Bromodichloromethane, PCE, 1,1-dichloro-ethane, and MTBE were detected in two samples; and benzene, trichlorofluoromethane, 1,1,1-trichloro-ethane, 1,1,2-trichloro-1,2,2trifluoroethane, 1-chloro-2-methyl benzene, 1,1dichloroethene, and acetone were each detected in one sample. Four detections of trichloromethane and dichloromethane, three detec-tions of PCE and 1,4dichlorobenzene, two detections of 1,2dimethylbenzene, and one detection of acetone and ethylbenzene were not evaluated for this report because these compounds were detected in field blanks associated with the environmental samples.

The VOCs detected in ground water come from a variety of sources that are difficult to determine. 1,2,4-trimethylbenzene could be from various urban land-use areas because of the variety of sources for this compound. Chloromethane, carbon disulfide, and iodomethane have natural and man-made sources that could contribute these compounds to the environment. Natural sources of chloromethane and carbon disulfide, such as fungus, forest fires, and volcanic gases (Harper, 1985; Inn and others, 1981; Palmer, 1976) could be contributing to the ground water. Chloromethane is manufactured for use as a refrigerant, methylating agent, dewaxing agent, and catalytic solvent in synthetic rubber production (U.S. Environmental Protection Agency, 1996). Rayon manufacturing is a source of manmade carbon disulfide (U.S. Environmental Protection Agency, 1994). Natural sources of iodomethane include microbial fermentation (Lovelock, 1975). Iodomethane is used as a methylating agent in organic synthesis and also is in exhaust gases from nuclear reactors (Howard, 1993).

MTBE is a fuel oxygenate added to improve air quality in metropolitan areas such as Phoenix (Pitzl, 1997). At least two wells owned by the City of Phoenix are no longer used because MTBE concentrations of 46 to $200 \mu \mathrm{g} / \mathrm{L}$ were measured in the ground water (Marsh, 1994). Sources of MTBE include point sources, such as leaking gasoline storage tanks, and nonpoint sources, such as precipitation and urban runoff (Squillace and others, 1998). 
At least 4 VOCs were detected in 3 of the 21 wells that had detectable concentrations of VOCs (table 16). The VOCs detected in these wells are either refrigerants, solvents, or are used to make solvents. These detections may be typical for small-capacity wells in the metropolitan Phoenix area and are similar to detections in wells in previous studies (Marsh, 1994). Although only 3 wells had detectable concentrations of 4 or more VOCs, the large variety of VOCs and the large area where samples contained VOCs, indicate that much of the ground water in the West Salt River Valley is affected by human activities.

At least one VOC was detected in samples from each of the nine wells in the agricultural LUS area in August 1997 and February 1998. Eight different VOCs (of the analytical suite of 86) were detected a total of 23 times in samples collected in August 1997 and February 1998. Some detections of 1,4-dichlorobenzene, chloromethane, and 1,2,4-trimethylbenzene were not considered in this report because these compounds were detected in the field blanks associated with the environmental samples. Four detections of 1,4-dichlorobenzene in samples collected in August and three detections in samples collected in February were affected by sample contamination. Nine detections of chloromethane in samples collected in August and two detections of 1,2,4-trimethylbenzene in samples collected in February were affected by sample contamination.

The most frequently detected VOC was trichloromethane, which was detected in each of the nine wells during both sampling periods. Effluent water from the 91st Avenue WWTP is applied to fields near the nine wells; therefore, outflow from the WWTP could be the source of the trichloromethane.
Bromodichloromethane often is detected along with trichloromethane because it is also a byproduct of chlorinated drinking water. Bromodichloromethane was detected in wells AG1 and AG5. The source of this compound probably is outflow from the WWTP.

The second most commonly detected VOC in the LUS wells was PCE, which was detected in three wells in August 1997 and in five wells in February 1998. Four of the five wells where the compound was detected in February 1998 are downgradient from a Comprehensive Environmental Response, Compensation, and Liability Act (CERCLA) site at the Phoenix-Goodyear Airport. Ground water at this site is contaminated with TCE and PCE (Marsh, 1994). PCE and TCE were detected during both sampling periods. The CERCLA site may be the source of PCE and TCE in these wells; however, local use of the compounds also could be a source. All but one of these detections was below the MRL.

Dibromochloropropane (DBCP) was detected in one well in February 1998. This compound, although considered a VOC, was the first pesticide detected in ground water in Arizona (Daniel and others, 1988) and was applied to soils in citrus and cotton fields from the 1950s through the 1980s to control nematodes (Daniel and others, 1988; Brew and Baker, 1987). Daniel and others (1988) reported many detections of this compound throughout the West Salt River Valley, but the compound was detected only once during sampling of wells in the West Salt River Valley in 1996-98. The reason for the lack of detections during 1996-98 is unclear; possible reasons include degradation of the parent compound and (or) differences in sampling locations.

Table 16. Volatile organic compounds in water from three wells having the highest number of detections, West Salt River Valley, Central Arizona Basins study area, 1996-98

\begin{tabular}{ccc}
$\begin{array}{c}\text { Well } \\
\text { number }\end{array}$ & $\begin{array}{c}\text { Number of } \\
\text { volatile organic } \\
\text { compounds detected }\end{array}$ & Volatile organic compounds \\
\hline W15 & 4 & Tetrachloroethene, trichloroethene, methyl tert-butyl ether, 1,1,2-trichloro-1,2,2-trifluoroethane \\
W16 & 5 & $\begin{array}{c}\text { Trichloroethene, methyl tert-butyl ether, bromodichloromethane, 1,1-dichloroethane, } \\
\text { trichlorofluoromethane } \\
\text { Trichloroethene, bromodichloromethane, 1,1-dichloroethane, 1,1-dichloroethene, } \\
1,1,1-\text { trichloroethane, carbon disulfide }\end{array}$ \\
W17 & 6 &
\end{tabular}




\section{Surface Water}

Surface-water samples were collected at three sites in the West Salt River Valley for VOC analyses-91st Avenue WWTP outfall near Phoenix, Gila River at Buckeye Canal (09514000), and the Hassayampa River near Arlington (table 15; fig. 14). A discussion of VOC results from the Hassayampa River is not included in this report because of the limited data available. None of the VOC concentrations in surface-water samples were above established limits of the USEPA (1996) or the State of Arizona (1996; table 13, this report).

Twenty-six VOCs (of the analytical suite of 86) were detected in 6 samples collected at the 91st Avenue WWTP from June 1996 through April 1997. All of these samples were collected during low-flow conditions. Some detections of methyl-benzene, chlorobenzene, ethylbenzene, 1,4-dichlorobenzene, carbon disulfide, 1,2,4-trimethylbenzene, acetone, tetrahydrofuran, and 1,3- and 1,4-dimethylbenzene are not considered in this report because the same compounds were detected in the field blanks associated with the environmental samples. Of the 26 compounds, 5 were detected in each of the 6 samplesbromodichloromethane, dibromochloromethane, trichloromethane, PCE, and bromochloromethane. Bromodichloromethane, chlorodibromomethane, and trichloromethane are trihalomethanes (THMs); the presence of these compounds is an indication that chlorination processes may be affecting the surfacewater quality (Squillace and others, 1999). PCE is a common VOC detected in ground water in this area. Bromochloromethane that is primarily used for organic synthesis was one of two VOCs identified as being unique in urban areas by Squillace and others (1999).

Fifteen VOCs (of the analytical suite of 86) were detected in the 5 samples collected at the Gila River at Buckeye Canal from June 1996 through April 1997. All of these samples were collected during low-flow conditions. Of the 15 compounds, trichloromethane was detected in all 5 samples. The fewer number of VOCs detected at this site compared with the number detected at the 91st Avenue WWTP indicates that the VOCs are volatilizing from the surface water as it travels downstream, or that dilution from other water inputs is affecting the concentration of these compounds.

Detections of VOCs in ground water and surface water are indications of the effects of human activities in the metropolitan Phoenix area on water quality. PCE in ground-water samples collected in the metropolitan Phoenix area and in surface-water samples collected at the 91st Avenue WWTP and the Gila River at Buckeye
Canal also is indicative of the effects of urban activities. These results are similar to results reported for the entire United States by Squillace and others (1999).

\section{SUMMARY AND CONCLUSIONS}

An assessment of pesticides and VOCs in ground water and surface water was completed in the CAZB study area for the USGS NAWQA program during 1996-98. Samples of ground water were collected from 121 wells, and samples of surface water were collected from 4 sites. Samples were collected from 121 wells and 3 surface-water sites for pesticide analyses, and samples were collected from 109 wells and 3 surfacewater sites for VOC analyses. Pesticides have been used extensively in localized areas of the CAZB since the 1940s. VOCs have been used in industrial areas of the CAZB and have been detected in ground water, primarily in urban areas.

The study design for ground water included sampling in three ground-water basins. The West Salt River Valley and the Upper Santa Cruz Basin were included because ground water in those areas is used in large quantities, and ground-water quality has been, or has the potential to be, affected by human activities. The Sierra Vista subbasin was included to represent a minimally developed basin for comparison to the West Salt River Valley and the Upper Santa Cruz Basin. Results from the Sierra Vista subbasin provided information that can be used for the design and assessment of future monitoring. Two different types of ground-water studies were completed-SUSs to generally characterize the ground-water quality of an aquifer, and a LUS to characterize the ground-water quality beneath an agricultural area. Wells sampled for the SUSs were existing wells used for industrial, irrigation, stock, and municipal and domestic water supplies. In the West Salt River Valley, 35 of 64 wells sampled were used for evaluation of pesticides and VOCs. In the Upper Santa Cruz Basin, 29 wells were used, and in the Sierra Vista subbasin, 19 wells were used. For the agricultural LUS, 9 monitoring wells were installed and were sampled twice.

Surface-water sites sampled for pesticides and (or) VOCs were on four rivers in the CAZB - the San Pedro, Gila, Hassayampa, and Santa Cruz Rivers. One site was on the San Pedro River. A group of three sites was sampled at the Gila River at Buckeye Canal; one site was sampled at the outfall of the 91st 
Avenue WWTP; and one site was sampled on the Hassayampa River near Arlington. Data from a group of four sites on the Santa Cruz River near Nogales that were sampled for another project also were used.

Detections of pesticides in ground water and surface water indicate that pesticides used in agricultural and urban areas are affecting the water quality. Twenty-eight percent of the wells sampled in the Upper Santa Cruz Basin and all surface-water samples collected from the Santa Cruz River and Nogales Wash had detectable concentrations of pesticides. Thirty-three percent of the wells sampled and nearly all the surface-water samples in the West Salt River Valley had detectable concentrations of pesticides. At least one pesticide was detected in all wells in the agricultural area of the West Salt River Valley. Pesticides applied for agricultural and urban uses were detected in the West Salt River Valley and the Upper Santa Cruz Basin. Pesticides detected in the Santa Cruz River and Nogales Wash were insecticides used in urban areas; the detections indicate effects of urban land use upstream. Pesticides detected in ground water from the agricultural area were those that were applied for agricultural and urban uses. Surface-water samples from the Gila River at Buckeye Canal and the Hassayampa River near Arlington contained pesticides used in agricultural and urban areas. Among the SUSs, the West Salt River Valley and the Upper Santa Cruz Basin had the largest number of detections per well for VOCs and pesticides. Ground water from each well in the agricultural LUS had one VOC detected. No pesticides were detected in ground-water or surfacewater samples from the Sierra Vista subbasin.

Detections of VOCs in ground water and surface water indicate that the areas sampled are affected by VOCs; however, the concentrations are less than established water-quality limits. In the Sierra Vista subbasin, 74 percent of the wells sampled contained VOCs. This result was much higher than expected because the subbasin has a small amount of urban land use, and it indicates that ground water in localized areas of this subbasin is affected by human activities. No surface-water samples were collected in the Sierra Vista subbasin for VOC analyses. Fifty-two percent of the wells sampled in the Upper Santa Cruz Basin contained at least one VOC. Detections of VOCs in every surface-water sample from the Santa Cruz River and Nogales Wash indicate the effects of urban activities upstream. Seventy percent of the wells sampled in the West Salt River Valley contained at least one VOC, and all wells in the agricultural area contained at least one VOC. Surface-water samples from the 91st Avenue WWTP and the Gila River at Buckeye Canal contained VOCs that can be related to urban land uses and well-chlorination processes.

Concentrations of pre-emergent pesticides detected at the Gila River at Buckeye Canal and the Hassayampa River near Arlington showed evidence of seasonal variability. Detections of DDE at the Gila River at Buckeye Canal also appeared to have some seasonal variability. Seasonal variability could be caused by irrigation-return flow that enters the canal from April through December. The detection of DDE in the Hassayampa River near Arlington throughout the year indicates that a transport mechanism operates all year.

Historical and present land-use activities appear to be affecting the quality of ground water and surface water in the areas sampled. The most prevalent indicator of the effects of historical agricultural land use are detections of DDE in ground water and surface water in the West Salt River Valley. Detections of atrazine and deethylatrazine in ground water from the Upper Santa Cruz Basin also indicate the effects of historical agriculture in this area. These compounds have not been used for decades in the areas where detections occurred. In the Tucson area, VOCs and pesticides associated with urban areas were detected in ground water. In the Nogales area, detections of VOCs and pesticides in ground water and surface water associated with urban areas indicate the effects of the urban land-use activities.

Detections of pesticides and VOCs in surface water and ground water are the most complex in the West Salt River Valley. The highest number of different pesticides and VOCs were detected in ground-water and surface-water samples from this basin. Detections of PCE and TCE in ground water and surface water are the most apparent indicators that urban land-use activities are affecting water quality.

The effects of present agriculture are best characterized by pesticides detected in ground water from the monitoring wells in the agricultural LUS and surface water from the Gila River at Buckeye Canal and the Hassayampa River near Arlington. Pesticides and VOCs detected at these sites also are indicators of urban land use. Pesticides primarily used in urban areas were detected in samples that also contained pesticides primarily used in agricultural areas. The complex mixture of pesticides in ground water and surface water is the direct result of the mixture of land uses and water-management practices in the West Salt River Valley. The reuse of water provides a mechanism for transport of pesticides and VOCs to areas that may not normally be affected by these compounds. A more 
detailed study of the sources of water is required to better determine whether urban or agricultural land-use activities are the sources of specific compounds.

Concentrations of pesticides and VOCs generally are less than the current established water-quality limits. Potential health effects from the occurrence of several compounds in the same water are not well known, and more than one compound was detected in several ground-water and surface-water samples in this study. The detections of pesticides and VOCs are important because they indicate that the ground-water and surface-water resources in the CAZB study area are susceptible to contamination by human activities.

\section{SELECTED REFERENCES}

Agency for Toxic Substances and Disease Registry, 1993, 1,4-Dichlorobenzene: Agency for Toxic Substances and Disease Registry, accessed November 18, 1999, at URL http://www.atsdr.cdc.gov.

Anderson, T.W., Freethey, G.W., and Tucci, Patrick, 1992, Geohydrology and water resources of alluvial basins in south-central Arizona and parts of adjacent states: U.S. Geological Survey Professional Paper 1406-B, 67 p.

Arizona Agricultural Statistics Service, 1991, 1990 Arizona agricultural statistics: Phoenix, Arizona, Agricultural Statistics Services report, $110 \mathrm{p}$.

Arizona Department of Economic Security, rev. March 27, 2001, April 1, 1990 and 2000 population for state/county/incorporated places: accessed May 22, 2001, at URL http://www.de.state.az.us/links/ economic/webpage/popweb/total2000.xls.

Arizona Department of Environmental Quality, 1997, Arizona toxic chemical release inventory report summary—Data collected for reporting year 1995: Arizona Department of Environmental Quality EQR 97-5, v.p.

Arizona Game and Fish Department, 1993, Arizona riparian inventory and mapping project: Phoenix, Arizona Game and Fish Department report, p. 41.

Barbash, J.E. and Resek, E.A., 1996, Pesticides in ground water-Distribution, trends, and governing factors: Chelsea, Michigan, Ann Arbor Press, Pesticides in the Hydrologic System series, v. 2, 590 p.

Brew, T.M., and Baker, P.B., 1987, A survey of historical and current agricultural pesticide use in Arizona: Phoenix, Arizona Department of Environmental Quality, Pesticide Coordinator's Office, $63 \mathrm{p}$.
Brown, S.L., 1993, Organochlorine pesticide levels associated with historic agricultural areas within the middle Gila River Basin: Tempe, Arizona, Arizona State University, master's thesis, $48 \mathrm{p}$.

City of Phoenix, 1999, City statistics-Community trends and profile: City of Phoenix, August 31, 1999, accessed September 23, 1999, at URL http://www.ci.phoenix. az.us/CITYGOV/stats.html.

Coes, A.L., Gellenbeck, D.J., and Towne, D.C., 1999, Ground-water quality in the Sierra Vista subbasin, Arizona, 1996-97: U.S. Geological Survey WaterResources Investigations Report 99-4056, 50 p.

Coes, A.L., Gellenbeck, D.J., Towne, D.C., and Freark, M.C., 2000, Ground-water quality in the Upper Santa Cruz Basin, Arizona, 1998: U.S. Geological Survey Water-Resources Investigations Report 00-4117, 85 p.

Connor, B.F., Rose, D.L., Noriega, M.C., Murtagh, L.K., and Abney, S.R., 1998, Methods of analysis by the U.S. Geological Survey National Water Quality Laboratory-Determination of 86 volatile organic compounds in water by gas chromatography/mass spectrometry, including detections less than reporting limits: U.S. Geological Survey Open-File Report 97-829, $78 \mathrm{p}$.

Cordy, G.E., 1994, National Water-Quality Assessment program-Central Arizona Basins: U.S. Geological Survey Fact Sheet 94-016, 2 p.

Cordy, G.E., Rees, J.A., Edmonds, R.J., Gebler, J.B., Wirt, Laurie, Gellenbeck, D.J., and Anning, D.W., 1998, Water-quality assessment of the Central Arizona Basins, Arizona and Northern Mexico-Environmental setting and overview of water quality: U.S. Geological Survey Water-Resources Investigations Report 984097, $72 \mathrm{p}$.

Damon, P.E., Lynch, D.J., and Shafiqullah, Mohammed, 1984, Cenozoic landscape development in the Basin and Range province of Arizona, in Smiley, T.L., Nations, J.D., Péwé, T.L., and Schafer, J.P., eds., Landscapes of Arizona: Lanham, Maryland, University Press of America, p. 175-206.

Daniel, D.L., Munson, B.E., and Graf, C.G., 1988, Groundwater contamination by pesticides in ArizonaAn overview, in Rasmussen, T.C., ed., Survival in the desert-Water quality and quantity issues into the 21 st century: Arizona Hydrological Society, Proceedings of the Third Annual Symposium, Casa Grande, Arizona, September 16, 1988, p. 72-100. 
Edmonds, R.J., and Gellenbeck, D.J., 2002, Ground-water quality in the West Salt River Valley, Arizona, 199698-Relations to hydrogeology, water use, and land use: U.S. Geological Survey Water-Resources Investigations Report 01-4126, 60 p.

Edwards, T.K., and Glysson, D.G., 1988, Field methods for measurement of fluvial sediment: U.S. Geological Survey Open-File Report 86-531, 118 p.

Extension Toxicology Network, 1996, Pesticide information profiles: accessed June 28, 1999, at URL http://ace.orst.edu/info/extoxnet.

Fenneman, N.M., 1931, Physiography of western United States: New York, McGraw-Hill, 534 p.

Gebler, J.B., 2000, Organochlorine compounds in streambed sediment and in biological tissue from streams and their relations to land use, central Arizona: U.S. Geological Survey Water-Resources Investigations Report 004041, 21 p.

Gellenbeck, D.J., and Coes, A.L., 1999, Ground-water quality in alluvial basins that have minimal urban development, south-central Arizona: U.S. Geological Survey Water-Resources Investigation Report 99-4005, $27 \mathrm{p}$.

Gilliom, R.J., Alley, W.M., and Gurtz, M.E., 1995, Design of the National Water-Quality Assessment programOccurrence and distribution of water-quality conditions: U.S. Geological Survey Circular 1112, 33 p.

Harper, D.B., 1985, Halomethane from halide ion-a highly efficient fungal conversion of environmental significance: Nature, v. 315, p. 55-57.

Helsel, D.R., and Hirsch, R.M., 1992, Statistical methods in water resources: New York, Elsevier, 529 p.

Hirsch, R.M., Alley, W.M., and Wilber, W.G., 1988, Concepts for a National Water-Quality Assessment program: U.S. Geological Survey Circular 1021, 42 p.

Hostetler, K.A., and Thurman, E.M., 1999, Determination of chloroacetanilide herbicide metabolites in water using high-performance liquid chromatography-diode array detection and high-performance liquid chromatography/mass spectrometry in Morganwalp, D.W. and Buxton, H.T., eds., U.S. Geological Survey Toxic Substances Hydrology ProgramProceedings of the technical meeting: Charleston, South Carolina, March 8-12, 1999, v. 2, Contamination of Hydrologic Systems and Related Ecosystems: U.S. Geological Survey Water-Resources Investigations Report 99-4018-B, p. 345-351.
Howard, P.H., 1993, Handbook of environmental fate and exposure data for organic chemicals-Volume IV, Solvents 2: Chelsea, Michigan, Lewis Publishers, Inc., p. 353-358.

Inn, E.C.Y., Vedder, J.F., Conon, E.P., and O'Hara, D., 1981, Gaseous constituents in the plume from eruptions of Mount Saint Helens: Science, v. 211, no. 20, p. 821-823.

Koterba, M.T., Wilde, F.D., and Lapham, W.W., 1995, Ground-water data-collection protocols for the National Water-Quality Assessment program-Collection and documentation of water-quality samples and related data: U.S. Geological Survey Open-File Report 95-399, $113 \mathrm{p}$.

Lapham, W.W., Wilde, F.D., and Koterba, M.T., 1995, Ground-water data-collection protocols and procedures for the National Water-Quality Assessment programSelection, installation, and documentation of wells, and collection of related data: U.S. Geological Survey Open-File Report 95-398, 69 p.

Larson, S.J., Capel, P.D., and Majewski, M.S., 1997, Pesticides in surface waters-Distribution, trends, and governing factors: Chelsea, Michigan, Ann Arbor Press, $390 \mathrm{p}$.

Lovelock, J.E., 1975, Natural halocarbons in the air and in the sea: Nature, v. 256, p. 193-194.

Maricopa Association of Governments, 1997, Socioeconomic projections: Maricopa Association of Governments interim report, $280 \mathrm{p}$.

Marsh, Diana, ed., 1994, Arizona water-quality assessment 1994: Phoenix, Arizona Department of Environmental Quality Report EQR 94-3, 246 p.

Morrow, W.S., Jr., 1999, Volatile organic compounds in ground water of the lower Illinois River Basin: U.S. Geological Survey Water-Resources Investigations Report 99-4229, 6 p.

Mueller, D.K., Martin, J.D., and Lopes, T.J., 1997, Qualitycontrol design for surface-water sampling in the National Water-Quality Assessment program: U.S. Geological Survey Open-File Report 97-223, 17 p.

Padgitt, M., 1997, Pesticides of agricultural resources and environmental indicators, agricultural handbook 712, chap. 3.2: Washington, D.C., U.S. Department of Agriculture, Economic Research Service, Natural Resources and Environment Division, p. 116-133.

Palmer, T.Y., 1976, Combustion sources of atmospheric chlorine: Nature, v. 263, p. 44-46.

Pitzl, M.J., 1997, Clean-burning gas likely to boost pump prices: Phoenix, Arizona, The Arizona Republic, July 2, 1997. 
Reynolds, S.J., 1988, Geologic map of Arizona: Arizona Geological Survey Map 26, scale 1:1,000,000.

Rose, D.L., and Schroeder, M.P., 1995, Methods of analysis by the U.S. Geological Survey National Water Quality Laboratory-Determination of volatile organic compounds in water by purge and trap capillary gas chromatography/mass spectrometry: U.S. Geological Survey Open-File Report 94-708, 26 p.

Sax, N.I., and Lewis, R.J., eds., 1987, Condensed chemical dictionary, (11th ed.): New York, Nostrand Reinhold Company.

Scott, J.C., 1990, Computerized stratified random siteselection approaches for design of a ground-waterquality sampling network: U.S. Geological Survey Water-Resources Investigations Report 90-4101, 109 p.

Shafiqullah, Mohammed, Damon, P.E., Lynch, D.J., Reynolds, S.J., and Rehrig, W.A., 1980, K-Ar geochronology and geologic history of southwestern Arizona and adjacent areas, in Jenny, J.P., and Stone, Claudia, eds., Studies in western Arizona: Arizona Geological Society Digest, v. 12, p. 201-260.

Shelton, L.R., 1994, Field guide for collecting and processing stream-water samples for the National Water-Quality Assessment program: U.S. Geological Survey Open-File Report 94-455, 42 p.

1997, Field guide for collecting samples for analysis of volatile organic compounds in stream water for the National Water-Quality Assessment program: U.S. Geological Survey Open-File Report 97-401, 14 p.

Smith, C.F., Duet, N.R., Fisk, G.G., McCormack, H.F., Partin, C.K., Pope, G.L., and Rigas, P.D., 1997, Water resources data Arizona water year 1996: U.S. Geological Survey Water-Data Report AZ-96-1, 328 p.

Squillace, P.J., Moran, M.J., Lapham, W.W., Price, C.V., Clawges, R.M., and Zogorski, J.S., 1999, Volatile organic compounds in untreated ambient ground water of the United States, 1985-95: Environmental Science and Technology, v. 33, no. 23, p. 4176-4187.

Squillace, P.J., Pankow, J.F., Korte, N.E., and Zogorski, J.S., 1996, Environmental behavior and fate of methyl tertbutyl ether (MTBE): U.S. Geological Survey Fact Sheet FS-203-96, 6 p.

State of Arizona, 1996, Water quality standards: Phoenix, State of Arizona, Arizona Administrative Code, Title 18, Chap. 11, p. 1-60.

Tadayon, Saeid, Duet, N.R., Fisk, G.G., McCormack, H.F., Pope, G.L., and Rigas, P.D., 1998, Water resources data Arizona water year 1997: U.S. Geological Survey Water-Data Report AZ-97-1, 416 p.
Tadayon, Saeid, Duet, N.R., Fisk, G.G., McCormack, H.F., Partin, C.K., Pope, G.L., and Rigas, P.D., 1999, Water resources data, Arizona, water year 1998: U.S. Geological Survey Water-Data Report AZ-98-1, 454 p.

Thomsen, B.W., and Baldys, Stanley, III, 1985, Groundwater conditions in and near the Gila River Indian Reservation, south-central Arizona: U.S. Geological Survey Water-Resources Investigations Report 854073, 2 sheets.

Thomsen, B.W., and Miller, B.H., 1991, Ground-water conditions in and near the Salt River Indian Reservation, south-central Arizona: U.S. Geological Survey Water-Resources Investigations Report 894176, 2 sheets.

Turner, E.M., Ward, M.W.T., Osborne, D.J., and Self, R., 1975 , Production of ethylene and other volatiles and changes in cellulase and laccase activities during the life cycles of the cultivated mushroom, Agarius bisporus: Journal of General Microbiology, v. 19, p. 167-176.

U.S. Bureau of the Census, 1991, Summary population and housing characteristics-Arizona: U.S. Department of Commerce, Bureau of the Census, 1990 Census of Population and Housing Report CPH-1-4, 5 p.

U.S. Department of Agriculture, 1995, Pesticide fact sheets: Information Ventures Inc., accessed August 23, 1999, at URL http://infoventures.com/e-hlth/pesticide/ pestfac.html.

U.S. Environmental Protection Agency, 1980, Ambient water-quality criteria for aldrin/dieldrin: U.S. Environmental Protection Agency, Criteria and Standards Division, 440/5-80-019, v.p. 1994, Chemicals in the environment: Carbon disulfide: U.S. Environmental Protection Agency, Office of Pollution Prevention and Toxics, EPA Document 749-F-94-008, accessed August 24,1999, at URL http://www.epa.gov/docs/opptintr/chemfact/ f_carbds.txt.

1995, Guidance for assessing chemical contaminant data for use in fish advisories, v.1-Fish sampling and analysis, 2 ed.: Washington, D.C., U.S. Environmental Protection Agency, Office of Water, EPA-823-R-95007, v.p.

1996, Drinking water regulations and health advisories: Washington, D.C., U.S. Environmental Protection Agency, Office of Water Report EPA 822B-96-002, $10 \mathrm{p}$.

1998, 1996 Toxics release inventory public data release: Washington, D.C., U.S. Environmental Protection Agency, Office of Pollution Prevention and Toxics, EPA 745-R-98-005. 
U.S. Geological Survey, 1999, The quality of our nation's waters-Nutrients and pesticides: U.S. Geological Survey Circular 1225, $82 \mathrm{p}$.

Water Resources Research Center, 1999, Water in the Tucson area-Seeking sustainability: Tucson, Arizona, University of Arizona, College of Agriculture, Water Resources Research Center, 155 p.

Werner, S.L., Burkhardt, M.R., and DeRusseau, S.N., 1996, Methods of analysis by the U.S. Geological Survey National Water Quality Laboratory-Determination of pesticides in water by Carbopak-B solid-phase extraction and high-performance liquid chromatography: U.S. Geological Survey Open-File Report 96-216, 42 p.
Western Technologies, 1997, Groundwater sampling-The Nogales Wash WQARF area: Tucson, Arizona, Arizona Department of Environmental Quality, $22 \mathrm{p}$.

Zaugg, S.D., Sandstrom, M.W., Smith, S.G., and Fehlberg, K.M., 1995, Methods of analysis by the U.S. Geological Survey National Water Quality Laboratory-Determination of pesticides in water by C-18 solid-phase extraction and capillary-column gas chromatography/mass spectrometry with selected-ion monitoring: U.S. Geological Survey Open-File Report 95-181, 49 p. 
Quality-assurance procedures were followed to minimize negative effects on environmental data. Quality-control samples were collected to quantify bias and variability in the environmental ground-water and surface-water data. Designs of the ground-water quality-assurance plan and quality-control sample collection for NAWQA are described in Koterba and others (1995). Designs of the surface-water qualityassurance plan and quality-control sample collection for NAWQA are described in Mueller and others (1997). Discussion of quality-assurance and qualitycontrol samples in this section is limited to those samples associated with pesticide and VOC data. Several types of quality-control samples were used to evaluate the quality of the environmental data (table 17).

Systematic contamination was identified if a compound was detected in more than 50 percent of the field blanks collected during 1996-98. Contamination of a specific compound was considered if the concentration in the environmental sample was less than 10 times the median concentration of the field blanks (Tadayon and others, 1999). Sample populations for ground water and surface water were considered separately. For this report, sample contamination of VOCs was considered chronologically using results from field blanks. If a VOC was detected in a field blank before collection of an environmental sample, detection of that VOC in the environmental sample was considered to be affected by sample contamination. The results of this approach do not take into account detection of a VOC in a source solution blank. Because it is possible that source solution blanks were contaminated during collection procedures in the field, it was difficult to determine the composition of the original source solution.

\section{Ground Water}

Quality-control samples used to evaluate pesticide and VOC data included field and equipment blanks, source solution blanks, PVC-wash samples, fieldmatrix spikes, laboratory-matrix spikes, laboratory-set spikes, and recoveries of surrogates (compounds at specific concentrations added to environmental samples to evaluate sampling and analytical processes). Source-solution blanks were used for VOC analyses only after June 1, 1997. To determine the quality of extraction techniques at the office laboratory, replicate pesticide samples were extracted and analyzed at the NWQL and compared to samples extracted at the office laboratory and analyzed at the NWQL.

Table 17. Quality-control samples collected in the Central Arizona Basins study area, 1996-98

\begin{tabular}{|c|c|}
\hline Quality-control sample & Description \\
\hline Field blank & $\begin{array}{l}\text { A blank solution subjected to the same aspects of sample collection, field processing, preservation, } \\
\text { transportation, and laboratory handling as an environmental sample. }\end{array}$ \\
\hline Equipment blank & $\begin{array}{l}\text { A blank solution processed in the office laboratory through all equipment used for collecting and processing } \\
\text { an environmental sample. }\end{array}$ \\
\hline Source-solution blank & A blank solution placed directly into sample containers in the field. \\
\hline PVC-wash sample & Tap water used to rinse PVC casing before installation in monitoring wells. \\
\hline Field-matrix spike & $\begin{array}{l}\text { Replicate ground-water or surface-water samples that have a specific amount of a mixture (spike solution) } \\
\text { added that contains several of the compounds to be analyzed. }\end{array}$ \\
\hline Laboratory-matrix spike & Environmental samples that were spiked and extracted at the NWQL. \\
\hline Laboratory-set spike & $\begin{array}{l}\text { Reagent-grade water that contains spike solutions. For pesticide analyses } 0.1 \mu \mathrm{L} \text { and } 0.5 \mu \mathrm{L} \text { were used for } \\
\text { GCMS and HPLC methods. }\end{array}$ \\
\hline Concurrent replicates & $\begin{array}{l}\text { Environmental samples collected from the same site by different sampling crews as close in time as } \\
\text { possible. }\end{array}$ \\
\hline Sequential replicates & A single environmental sample collected at one time that is split into multiple samples. \\
\hline Canister blank & VOC-free water from the canister used to hold the VOC surface-water sampler. \\
\hline Sampler blank & $\begin{array}{l}\text { A blank collected after the canister blank. The sampler is submerged into the canister and a sample is } \\
\text { collected from the sampler for VOC analyses. }\end{array}$ \\
\hline Trip blank & $\begin{array}{l}\text { A blank solution put in a sample container at the NWQL and kept with the set of environmental samples } \\
\text { during storage, transport, and analysis. }\end{array}$ \\
\hline
\end{tabular}


Field blanks for ground water were collected at 16 and 18 sites for analyses of pesticides using HPLC and GCMS methods, respectively (table 18). Field blanks were collected by passing pesticide-free water through the sampling equipment subsequent to collection of the environmental samples and subsequent to cleaning of the sampling equipment. No pesticide compounds were detected in any of the 16 field blanks collected for HPLC analyses. GCMS methods detected DDE and dacthal at concentrations less than the MRLs at two sites and one site, respectively. These detections do not constitute systematic contamination and did not affect the ground-water pesticide data used in this report.

A total of 18 VOCs were detected in 18 groundwater field blanks; all 18 compounds were found in at least 1 of the 10 source-solution samples analyzed after June 1, 1997. Other than these detections, no VOCs were detected in more than 50 percent of field blanks (tables 19 and 20). Analysis of the occurrence of VOCs in field blanks and environmental samples resulted in the elimination of detections of trichloromethane, ethylbenzene, chloromethane, dichloromethane, tetrachloroethene, 1,4dichlorobenzene, 1,2-dimethyl-benzene, 1,2,4trimethylbenzene, and acetone from consideration during interpretation. Specific detections are discussed in the text of the report where appropriate.

Equipment blanks were collected from the Bennett submersible pump and other equipment used to sample ground water from the monitoring wells installed for the agricultural LUS. These samples were collected in the office before each of the sampling efforts in August 1997 and February 1998 to identify constituents or compounds that may be introduced to the environmental samples from the equipment. No pesticide compounds were detected in either of the equipment blanks (table 18). One VOC-1,2,4trimethylbenzene-was detected in equipment blanks and the accompanying source-solution blanks. These detections did not affect interpretation of the environmental data.

Two PVC-wash samples had detectable concentrations of 25 different VOCs (table 21). Of these compounds, five also were detected in environmental samples from the nine monitoring wells. Of these five compounds, three- bromodichloromethane, chloromethane, and carbon disulfidewere detected three times or less in environmental samples from the monitoring wells and are not considered an indication of sample contamination. Trichloromethane, which was detected in ground water from each of the nine monitoring wells and PVC-wash samples, was not detected in any field blank. Detections of trichloromethane in environmental samples from the monitoring wells, therefore, are not the result of contamination.

Field-matrix spikes were collected to provide information on the bias and variability of results due to field and laboratory techniques, the stability of compounds during typical sample holding times, and interference from the ground-water composition (matrix effects). The ground-water samples were spiked in the field during sample preservation.

Field-matrix spikes for pesticides in ground-water samples were collected and analyzed by HPLC and GCMS using $100 \mu \mathrm{L}$ of spike solution. Median recoveries ranged from 56 to 158 percent for fieldmatrix spikes for GCMS analyses and from 14 to 101 percent for HPLC analyses (table 22). Median recoveries for 4 laboratory-matrix spikes for GCMS analyses ranged from 49 to 136 percent and for HPLC analyses ranged from 50 to 120 percent (table 22). The laboratory-set spikes indicated that the analytical performance of 10 pesticides is considered to be poor (Jeff Martin, hydrologist, USGS, written commun., 1999). For these 10 pesticides (table 22), recoveries of compounds in the spike solution are either extremely low or extremely high compared to the ideal recovery of 100 percent.

Comparison of the median recovery of the fieldmatrix spikes to the laboratory-matrix spikes and the laboratory-set spikes indicates any biases that may have occurred in the pesticide data because of field techniques and (or) matrix effects. Bias in the data was identified by comparing the difference between median recoveries for field-matrix spikes to the interquartile range (IQR) of the laboratory-matrix spikes and laboratory-set spikes. Bias due to field techniques was identified when the difference between median recoveries for field-matrix spikes and laboratory-matrix spikes exceeded the IQR of the laboratory-matrix spikes. The results for twenty-two compounds were considered to have some bias owing to field techniques (table 22). Bias because of matrix effects was identified when the difference between median recoveries for the field-matrix spikes and laboratory-set spikes was greater than the IQR of the laboratory-set spikes. Twenty compounds were considered to have some bias due to matrix effects. No adjustments were made to the 
environmental data to compensate for any bias, but these results were considered during interpretation of the environmental data.

Surrogate compounds were added to environmental and quality control samples to provide information about matrix effects and gross sample-processing error. The surrogate compounds used are not normally found in environmental samples, but do behave like some of the compounds in the analysis. For interpretation, only results for surrogate compounds in environmental samples are discussed because they represent the variety of water types collected. For samples analyzed for pesticide compounds using GCMS, three surrogate compounds were used-diazinon-d10, terbuthylazine, and alpha-D6-1,2,3,4,5,6-hexachlorocyclohexane. In environmental ground-water samples collected during 1996-98, the median recoveries were 99 to 108 percent (table 23). For samples analyzed for pesticides using the HPLC method, one surrogate compound was used-4-bromo-3,5-dimethyl phenyl-nmethylcarbamate. The median recovery of this surrogate in all ground-water samples was 89.5 percent. No adjustments were made to groundwater pesticide data on the basis of surrogate recoveries.

Two types of spike solutions were used during the sampling period for VOC field-matrix spikes. Of the 26 field-matrix spikes completed for ground-water samples during 1996-98, 22 were spiked with a solution that contained 13 VOC compounds and 4 were spiked with a solution that contained 87 VOC compounds (table 24). Field-matrix spikes collected prior to June 1997 were spiked with $100 \mu \mathrm{L}$ of spike solution; after June 1997, $20 \mu \mathrm{L}$ of spike solution was used. The volume of spike solution was reduced to better evaluate the field and laboratory techniques at lower concentrations. Median recoveries of VOCs from field-matrix spikes ranged from 38 to 118 percent for individual compounds. One sample was spiked at the NWQL for 13 VOCs; recoveries for that sample were within one standard deviation of the median values for field-matrix spikes. Median recoveries of 2-propenal, chloromethane, and dichlorodifluoromethane in the field-matrix spikes were less than 60 percent. These recoveries indicate a possible negative bias in the data for these compounds owing to degradation or matrix effects. These compounds may not have been detected in ground-water samples if present in low concentrations. No adjustments were made to groundwater VOC data to compensate for this possible bias.
Three surrogate compounds were added to all VOC samples at the NWQL to evaluate matrix effects of sample-processing errors-1,4-bromofluorobenzene, 1,2-dichloroethane-d4, and toluene-d8. The median recoveries for these three compounds ranged from 95 to 106 percent (table 23). The recoveries for the VOC surrogate compounds indicate that the groundwater VOC data are acceptable.

\section{Surface Water}

Quality-control samples collected to evaluate surface-water pesticide data included field and equipment blanks, replicate samples, and two fieldmatrix spikes. Surface-water VOC data were evaluated using sampler blanks, canister blanks, source-solution blanks, and a trip blank. Quality-control samples for surface-water VOC data were collected at the 91st Avenue WWTP.

Seven pesticide field blanks were collected at four surface-water sites where pesticide samples were collected (table 25). Field blanks were collected before the environmental sample was collected by passing pesticide-free water through all surface-water sampling equipment. One field blank collected at the Hassayampa River near Arlington had a detectable concentration of DDE. No systematic contamination was identified for surface-water pesticide data.

Blank samples for VOCs in surface water included one sampler blank collected in June 1996 and a combination sampler and canister blank collected in January 1997. A source-solution blank also was collected in association with the sampler blank collected in the office laboratory in June 1996. In June 1996, all four compounds detected in the sampler blank collected also were detected in the associated sourcesolution blank (table 26). In January 1997, a sampler blank and a canister blank were collected. Analyses from the sampler and canister blanks were used to determine which surface-water samples were affected by sample contamination. Sample contamination was identified if a VOC was detected in a sampler or canister blank and if the concentration in the environmental samples was less than 10 times the concentration in the sampler or canister blank. Some detections of methylbenzene, chlorobenzene, ethylbenzene, 1,4-dichlorobenzene, carbon disulfide, 1,2,4-trimethylbenzene, acetone, and 1,3- and 1,4-dimethylbenzene were affected by sample contamination. 
Surface-water field-matrix spikes were collected to evaluate pesticide and VOC sampling and analytical procedures. One field-matrix spike was collected to evaluate the surface-water VOC data, and two fieldmatrix spikes were collected to evaluate the surfacewater pesticide data.

The field-matrix spikes for pesticides in surfacewater samples included both analytical methodsHPLC and GCMS — using $100 \mu \mathrm{L}$ of spike solution. Median recoveries for the GCMS method ranged from 0 to 318 percent, and median recoveries for the HPLC method ranged from 0 to 136 percent (table 27).

Measurable recoveries of 75 compounds occurred in at least one field-matrix spike. When the median recoveries for the 75 compounds were compared to the median recoveries for the laboratory-set spikes (table 22), recoveries for 41 compounds were outside the IQR for the laboratory-set spikes. These results indicate that a bias owing to matrix effects could be affecting the recoveries of those compounds in surfacewater samples. The small number of field-matrix spikes limits an evaluation of the bias; no adjustments were made to surface-water pesticide data on the basis of these results.

Median recoveries of surrogate pesticide compounds in surface-water samples analyzed using the GCMS method indicate no systematic problems with matrix effects or sample-processing errors. The median recoveries for the three surrogate compounds range from 100 to 151 percent (table 23). Recovery of the surrogate compound for pesticides analyzed using the HPLC method was 93 percent; this result indicates that the environmental samples are likely not affected by matrix effects or sample-processing errors. The surrogate compound for the HPLC method was added to 52 of the 73 samples analyzed for pesticides between December 1996 and April 1998; therefore, these results may not be indicative of all the environmental surfacewater samples collected.

The field-matrix spikes for VOCs used $100 \mu \mathrm{L}$ of spike solution that contained 13 VOC compounds (table 24). Recoveries for these 13 compounds ranged from 27 to 86 percent. Recoveries for all 13 compounds were less than the median recoveries for ground-water field-matrix spikes. Recoveries for most compounds were within the IQR of the field-matrix spikes for ground water except 1,2-dichloroethane and tribromomethane (table 24). This limited data could indicate that there is a bias towards lower values in surface-water samples analyzed for VOCs. Some VOCs may not have been detected if present in low concentrations in some surface-water samples. Because only one VOC field-matrix spike was collected, this interpretation was not applied to environmental surface-water VOC data.

Recoveries for the three surrogate compounds used for VOC analyses indicate that matrix effects and sample-processing errors did not affect the environmental surface-water VOC data. The median recoveries for the three surrogate compounds range from 99.5 to 105 percent (table 23), and are considered acceptable in this report.

Replicate samples were collected and analyzed to evaluate the variability in pesticide compound concentrations caused by differences in sample collection, processing procedures, and analytical techniques. Concurrent replicates were collected at the Gila River at Buckeye Canal and the Hassayampa River near Arlington during March and July 1997. Each of the concurrent samples was split into two split-replicate samples (table 28). Evaluation of these replicates identified variability due to sample collection procedures by comparing the difference between split replicates to the difference between concurrent replicates. Two additional split replicates were collected at both sites (table 29). Three of the splitreplicate samples were used to determine if any difference existed between samples extracted at the NWQL and samples extracted at the office laboratory. Only one compound was detected in the replicates of those analyzed by the HPLC method; because of the limited data, the performance of this method could not be evaluated.

For evaluation of the concurrent replicates, detections of 17 compounds can be considered. Median values of differences between concurrent replicate samples ranged from 2 to 18 percent. Variability due to differences in sampling techniques can be evaluated using data from concurrent replicates. For evaluation of split replicates, detections of 21 compounds can be evaluated. Median values of differences between split replicates ranged from 3 to 33 percent. Variability due to differences in processing and analytical procedures can be evaluated using data from split replicates. For the 17 compounds that were detected in both types of replicate samples, variability due to differences in processing and analytical procedures appears to be greater than variability due to sampling techniques. For most compounds, the median percent difference between concurrent and replicate samples was less than 
5 percent; the difference was more than 5 percent for one compound-pendimethalin. For this compound, some variation in concentrations may be due to differences in sampling procedures.

For split-replicate samples, six compounds have median differences greater than 10 percentcyanazine, DDE, deethylatrazine, gamma-HCH, prometon, and simazine. Variation in the concentrations of these compounds could be due to differences in processing and analytical procedures; this was considered during interpretation of these data. By comparing concentrations of these compounds in samples from the Hassayampa River near Arlington with concentrations in samples from the Gila River at Buckeye Canal, it was determined that processing and analytical procedures were the causes for differences.
Two split replicates were collected at the $91 \mathrm{st}$ Avenue WWTP to determine variability of VOC concentrations. Concentration data for 36 of the 86 compounds analyzed could be used in this analysis (table 26). The median percent differences for the 14 compounds that have measurable, nonestimated concentrations ranged from 2 to 178 . The range for all compounds with measurable values (both estimated and nonestimated) was 0 to 186 percent. These results indicate that variability in the environmental samples may be due to differences in sampling and analytical procedures and not variations in environmental conditions. Because only 14 VOCs in two split replicates could be evaluated, it is difficult to determine the application of these results to the environmental data. 


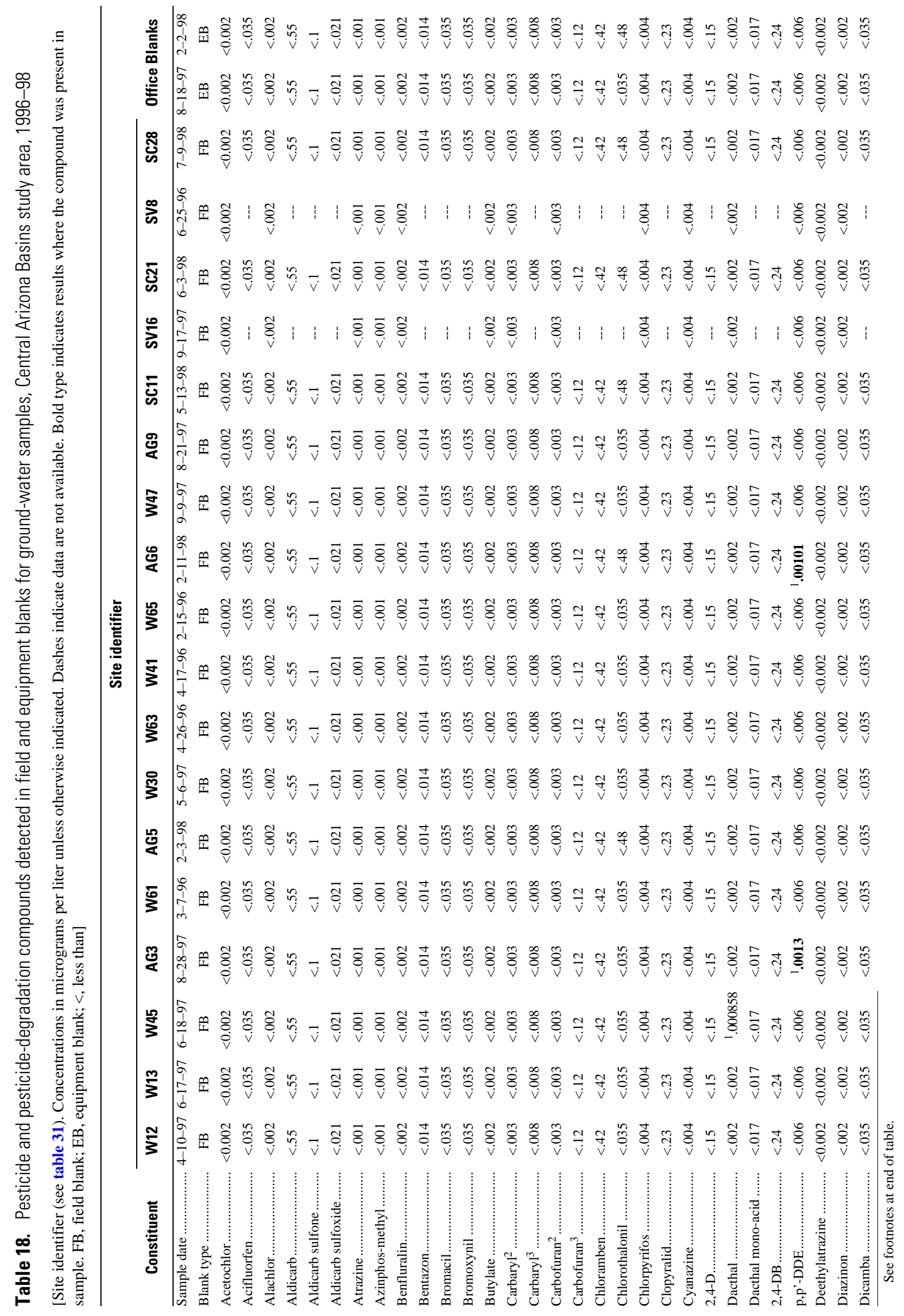




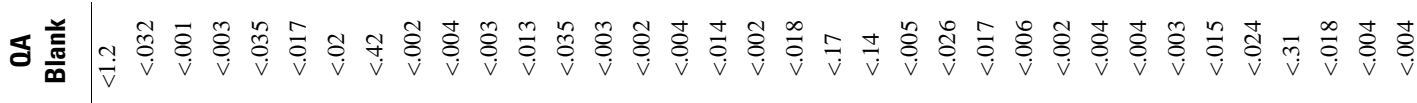

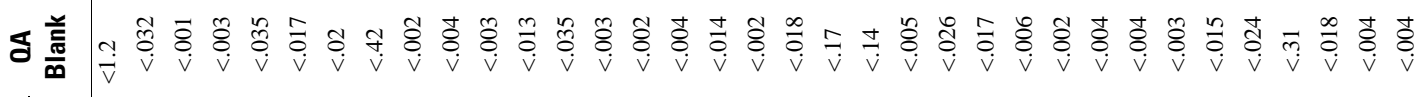

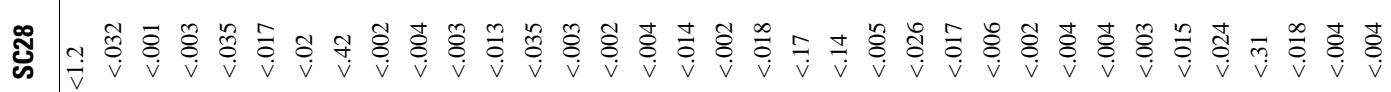

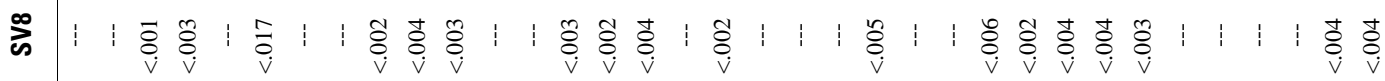
可

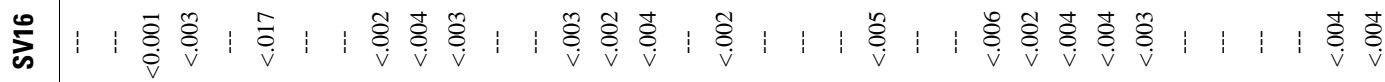

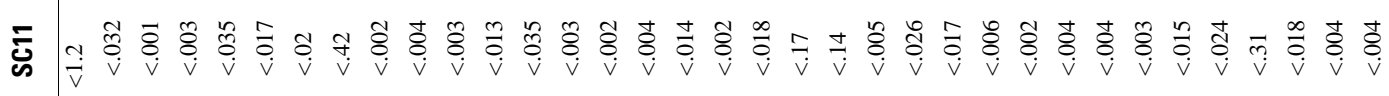

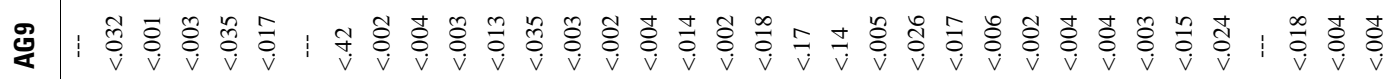

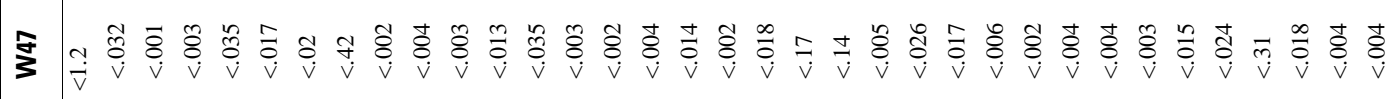

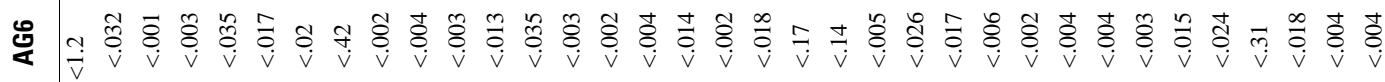

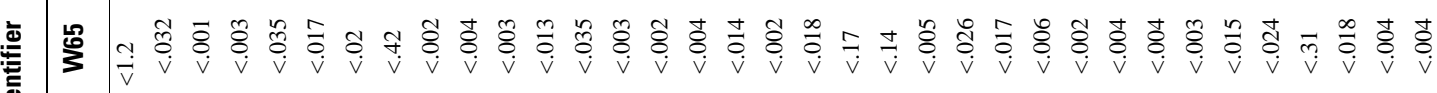

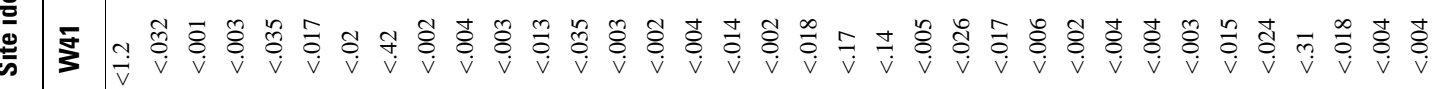

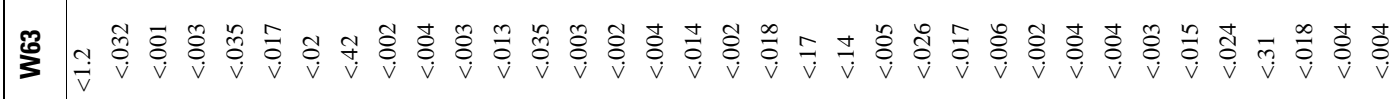

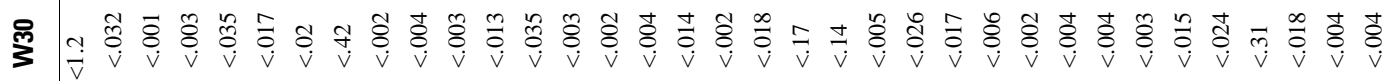

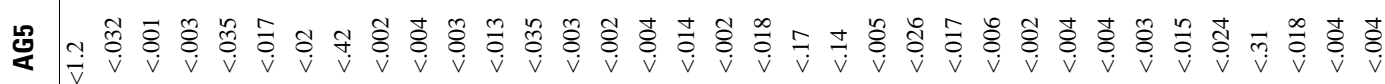

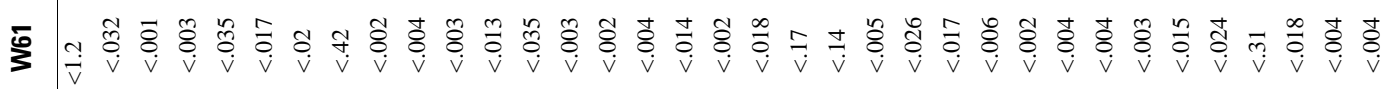

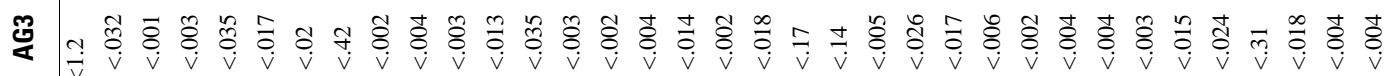

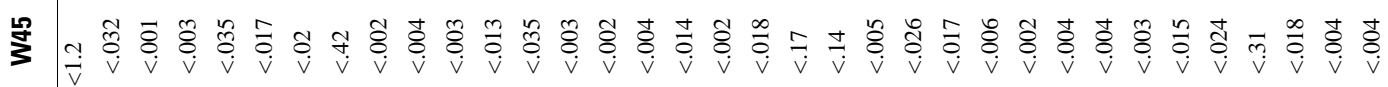

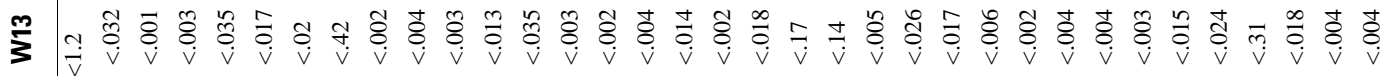

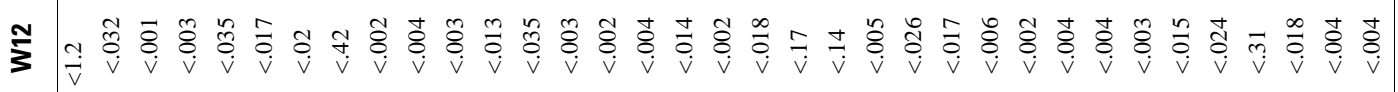




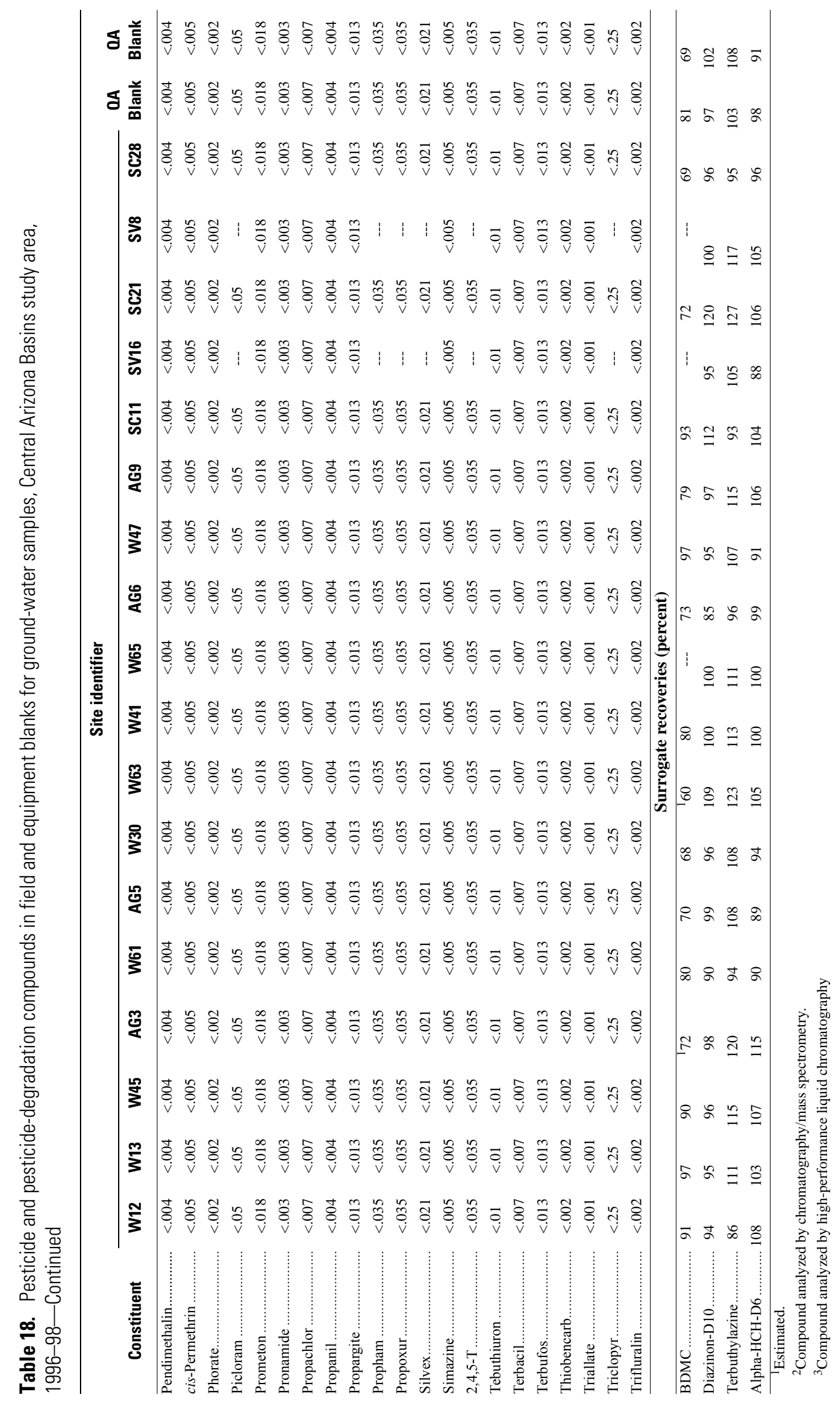


Table 19. Volatile organic compounds detected in field blanks for ground-water samples collected March 7 to September 17, 1996, Central Arizona Basins study area

[Dashes indicate data are not available. Bold type indicates results where the compound was present in sample.]

\begin{tabular}{|c|c|c|c|c|c|c|}
\hline \multirow[b]{2}{*}{ Constituent } & \multicolumn{6}{|c|}{ Well name } \\
\hline & W65 & W61 & W41 & SV8 & SV13 & SV16 \\
\hline Sample date & $2-15-96$ & $3-7-96$ & $4-17-96$ & $6-25-96$ & $8-14-96$ & $9-17-96$ \\
\hline Source solution lot number & 95228 & 95228 & 95228 & 95228 & 95304 & 95304 \\
\hline Dibromomethane & $<0.2$ & $<0.2$ & $<0.1$ & $<0.1$ & $<0.1$ & $<0.1$ \\
\hline Bromodichloromethane & $<.2$ & $<.2$ & ${ }^{1} .01$ & $<.1$ & $<.1$ & $<.1$ \\
\hline Tetrachloromethane & $<.2$ & $<.2$ & $<.05$ & $<.05$ & $<.05$ & $<.05$ \\
\hline 1,2-Dichloroethane & $<.2$ & $<.2$ & $<.05$ & $<.05$ & $<.05$ & $<.05$ \\
\hline Tribromomethane & $<.2$ & $<.2$ & $<.2$ & $<.2$ & $<.2$ & $<.2$ \\
\hline Dibromochloromethane & $<.2$ & $<.2$ & $<.1$ & $<.1$ & $<.1$ & $<.1$ \\
\hline Trichloromethane & $<.2$ & $<.2$ & ${ }^{1} .04$ & ${ }^{1} .04$ & ${ }^{1} .12$ & $<.08$ \\
\hline Methylbenzene & $<.2$ & $<.2$ & $<.05$ & $<.05$ & $<.05$ & $<.05$ \\
\hline Benzene & $<.2$ & $<.2$ & $<.05$ & $<.05$ & $<.05$ & $<.05$ \\
\hline 2-Propenal & --- & --- & $<2$ & $<2$ & $<2$ & $<2$ \\
\hline 2-Propenenitrile & -- & --- & $<2$ & $<2$. & $<2$ & $<2$ \\
\hline Chlorobenzene & $<.2$ & $<.2$ & ${ }^{1} .01$ & $<.05$ & $<.05$ & $<.05$ \\
\hline Chloroethane & $<.2$ & $<.2$ & $<.1$ & $<.1$ & $<.1$ & $<.1$ \\
\hline Ethylbenzene & $<.2$ & $<.2$ & $<.05$ & $<.05$ & $<.05$ & $<.05$ \\
\hline 1,1,1,2,2,2-Hexa-chloroethane & --- & --- & $<.05$ & $<.05$ & $<.05$ & $<.05$ \\
\hline Bromomethane & $<.2$ & $<.2$ & $<.1$ & $<.1$ & $<.1$ & $<.1$ \\
\hline Chloromethane & $<.2$ & $<.2$ & $<.2$ & $<.2$ & $<.2$ & $<.2$ \\
\hline Dichloromethane & $<.2$ & $<.2$ & $<.1$ & $<.1$ & $<.1$ & $<.1$ \\
\hline Tetrachloroethene & $<.2$ & $<.2$ & $<.05$ & $<.05$ & $<.05$ & $<.05$ \\
\hline Trichlorofluoromethane & $<.2$ & $<.2$ & $<.1$ & $<.1$ & $<.1$ & $<.1$ \\
\hline 1,1-Dichloroethane & $<.2$ & $<.2$ & $<.05$ & $<.05$ & $<.05$ & $<.05$ \\
\hline 1,1-Dichloroethene & $<.2$ & $<.2$ & $<.1$ & $<.1$ & $<.1$ & $<.1$ \\
\hline 1,1,1-Trichloroethane & $<.2$ & $<.2$ & $<.05$ & $<.05$ & $<.05$ & $<.05$ \\
\hline 1,1,2-Trichloroethane & $<.2$ & $<.2$ & $<.1$ & $<.1$ & $<.1$ & $<.1$ \\
\hline $1,1,2,2$-Tetrachloroethane & $<.2$ & $<.2$ & $<.1$ & $<.1$ & $<.1$ & $<.1$ \\
\hline 1,2-Dichlorobenzene & $<.2$ & $<.2$ & $<.05$ & $<.05$ & $<.05$ & $<.05$ \\
\hline 1,2-Dichloropropane & $<.2$ & $<.2$ & ${ }^{1} .02$ & $<.05$ & $<.05$ & $<.05$ \\
\hline trans-1,2-Dichloroethene & $<.2$ & $<.2$ & $<.05$ & $<.05$ & $<.05$ & $<.05$ \\
\hline 1,2,4-Trichlorobenzene & $<.2$ & $<.2$ & $<.2$ & $<.2$ & $<.2$ & $<.2$ \\
\hline 1,3-Dichlorobenzene & $<.2$ & $<.2$ & $<.05$ & $<.05$ & $<.05$ & $<.05$ \\
\hline 1,4-Dichlorobenzene & $<.2$ & $<.2$ & $<.05$ & $<.05$ & $<.05$ & $<.05$ \\
\hline Dichlorodifluoromethane & $<.2$ & $<.2$ & $<.2$ & $<.2$ & $<.2$ & $<.2$ \\
\hline Naphthalene & $<.2$ & $<.2$ & $<.2$ & $<.2$ & $<.2$ & $<.2$ \\
\hline trans-1,3-Dichloropropane & $<.2$ & $<.2$ & $<.1$ & $<.1$ & $<.1$ & $<.1$ \\
\hline cis-1,3-Dichloropropene & $<.2$ & $<.2$ & $<.1$ & $<.1$ & $<.1$ & $<.1$ \\
\hline Chloroethene & $<.2$ & $<.2$ & $<.1$ & $<.1$ & $<.1$ & $<.1$ \\
\hline
\end{tabular}

See footnote at end of table. 
Table 19. Volatile organic compounds detected in field blanks for ground-water samples collected March 7 to September 17, 1996, Central Arizona Basins study area—Continued

\begin{tabular}{|c|c|c|c|c|c|c|}
\hline \multirow[b]{2}{*}{ Constituent } & \multicolumn{6}{|c|}{ Well name } \\
\hline & W65 & W61 & W41 & SV8 & SV13 & SV16 \\
\hline Trichloroethene & $<.2$ & $<.2$ & $<.05$ & $<.05$ & $<.05$ & $<.05$ \\
\hline Hexachlorobutadiene & $<.2$ & $<.2$ & $<.2$ & $<.2$ & $<.2$ & $<.2$ \\
\hline Methyl acrylate & --- & --- & $<2$ & $<2$ & $<2$ & $<2$ \\
\hline 1,2,3,4-Tetramethylbenzene & --- & --- & $<.05$ & $<.05$ & $<.05$ & $<.05$ \\
\hline 1,2,3,5-Tetramethylbenzene & -- & -- & $<.05$ & $<.05$ & $<.05$ & $<.05$ \\
\hline Bromoethene & --- & --- & $<.1$ & $<.1$ & $<.1$ & $<.1$ \\
\hline Ethyl-tert-butyl ether & --- & --- & $<0.1$ & $<0.1$ & $<0.1$ & $<0.1$ \\
\hline tert-Amyl methyl ether & --- & -- & $<.1$ & $<.1$ & $<.1$ & $<.1$ \\
\hline trans-1,4-Dichloro-2-butene & --- & --- & $<5$ & $<5$ & $<5$ & $<5$ \\
\hline Ethyl methacrylate & --- & --- & $<1$. & $<1$ & $<1$. & $<1$. \\
\hline Carbon disulfide & --- & --- & ${ }^{1} .006$ & $<.05$ & $<.05$ & $<.05$ \\
\hline Vinyl acetate & --- & --- & $<5$. & $<5$ & $<5$ & $<5$ \\
\hline cis-1,2-Dichloroethene & $<0.2$ & $<0.2$ & $<.05$ & $<.05$ & $<.05$ & $<.05$ \\
\hline 2-Hexanone & ---- & ---- & $<5$. & $<5$ & $<5$. & $<5$. \\
\hline Ethenylbenzene & $<.2$ & $<.2$ & $<.05$ & $<.05$ & $<.05$ & $<.05$ \\
\hline 1,2-Dimethylbenzene & --- & --- & $<.05$ & $<.05$ & $<.05$ & $<.05$ \\
\hline 1,1-Dichloropropene & $<.2$ & $<.2$ & $<.05$ & $<.05$ & $<.05$ & $<.05$ \\
\hline 2,2-Dichloropropane & $<.2$ & $<.2$ & $<.05$ & $<.05$ & $<.05$ & $<.05$ \\
\hline 1,3-Dichloropropane & $<.2$ & $<.2$ & $<.05$ & $<.05$ & $<.05$ & $<.05$ \\
\hline 2-Ethyltoluene & -- & -- & $<.05$ & $<.05$ & $<.05$ & $<.05$ \\
\hline 1,2,3-Trimethylbenzene & --- & --- & $<.05$ & $<.05$ & $<.05$ & $<.05$ \\
\hline 1,2,4-Trimethylbenzene & $<.2$ & $<.2$ & ${ }^{1} .01$ & ${ }^{1} .05$ & .11 & ${ }^{1} .05$ \\
\hline (1-Methylethyl)benzene & $<.2$ & $<.2$ & $<.05$ & $<.05$ & $<.05$ & $<.05$ \\
\hline n-Propylbenzene & $<.2$ & $<.2$ & $<.05$ & $<.05$ & $<.05$ & $<.05$ \\
\hline 1,3,5-Trimethylbenzene & $<.2$ & $<.2$ & $<.05$ & $<.05$ & $<.05$ & $<.05$ \\
\hline 1-Chloro-2-methylbenzene & $<.2$ & $<.2$ & $<.05$ & $<.05$ & $<.05$ & $<.05$ \\
\hline 1-Chloro-4-methylbenzene & $<.2$ & $<.2$ & $<.05$ & $<.05$ & $<.05$ & $<.05$ \\
\hline Bromochloromethane & $<.2$ & $<.2$ & $<.1$ & $<.1$ & $<.1$ & $<.1$ \\
\hline n-Butylbenzene & $<.2$ & $<.2$ & $<.05$ & $<.05$ & $<.05$ & $<.05$ \\
\hline (1-Methylpropyl)benzene & $<.2$ & $<.2$ & $<.05$ & $<.05$ & $<.05$ & $<.05$ \\
\hline (1,1-Dimethylethyl)benzene & $<.2$ & $<.2$ & $<.05$ & $<.05$ & $<.05$ & $<.05$ \\
\hline 1-Isopropyl-4-methylbenzene & $<.2$ & $<.2$ & $<.05$ & $<.05$ & $<.05$ & $<.05$ \\
\hline Iodomethane & --- & --- & $<.05$ & $<.05$ & $<.05$ & $<.05$ \\
\hline 1,2,3-Trichloropropane & $<.2$ & $<.2$ & $<.2$ & $<.2$ & $<.2$ & $<.2$ \\
\hline 1,1,1,2-Tetrachloroethane & $<.2$ & $<.2$ & $<.05$ & $<.05$ & $<.05$ & $<.05$ \\
\hline 1,2,3-Trichlorobenzene & $<.2$ & $<.2$ & $<.2$ & $<.2$ & $<.2$ & $<.2$ \\
\hline 1,2-Dibromomethane & $<.2$ & $<.2$ & $<.1$ & $<.1$ & $<.1$ & $<.1$ \\
\hline 1,1,2-Trichloro-1,2,2-trifluoroethane & $<.2$ & $<.2$ & $<.05$ & $<.05$ & $<.05$ & $<.05$ \\
\hline Methyl tert-butyl ether & $<.2$ & $<.2$ & $<.1$ & $<.1$ & $<.1$ & $<.1$ \\
\hline 3-Chloro-1-propene & --- & -- & $<.1$ & $<.1$ & $<.1$ & $<.1$ \\
\hline
\end{tabular}


Table 19. Volatile organic compounds detected in field blanks for ground-water samples collected March 7 to September 17, 1996, Central Arizona Basins study area—Continued

\begin{tabular}{|c|c|c|c|c|c|c|}
\hline \multirow[b]{2}{*}{ Constituent } & \multicolumn{6}{|c|}{ Well name } \\
\hline & W65 & W61 & W41 & SV8 & SV13 & SV16 \\
\hline 4-Methyl-2-pentanone & --- & $\begin{array}{c}-- \\
\end{array}$ & $<5$. & $<5$ & $<5$. & $<5$. \\
\hline Xylenes & $<.2$ & $<.2$ & --- & --- & --- & --- \\
\hline Acetone & ---- & ---- & 8.3 & $<5$ & $<5$ & $<5$. \\
\hline Bromobenzene & $<.2$ & $<.2$ & $<.05$ & $<.05$ & $<.05$ & $<.05$ \\
\hline Diethyl ether & --- & --- & $<.1$ & $<.1$ & $<.1$ & $<.1$ \\
\hline Diisopropyl ether & --- & --- & $<.1$ & $<.1$ & $<.1$ & $<.1$ \\
\hline Methyl acrylonitrile & --- & --- & $<2$ & $<2$ & $<2$ & $<2$ \\
\hline 2-Butanone & --- & --- & ${ }^{1} .4$ & $<5$ & $<5$. & $<5$. \\
\hline Methyl methacrylate & --- & --- & $<1$. & $<1$ & $<1$. & $<1$. \\
\hline Tetrahydrofuran & --- & --- & ${ }^{1} .6$ & $<5$ & $<5$. & $<5$ \\
\hline 1,2-Dibromo-3-chloropropane & $<1$. & $<1$. & $<.5$ & $<.5$ & $<.5$ & $<.5$ \\
\hline $\begin{array}{l}\text { 1,3-Dimethylbenzene and } \\
\text { 1,4-Dimethylbenzene }\end{array}$ & --- & --- & $<.05$ & $<.05$ & $<.05$ & $<.05$ \\
\hline
\end{tabular}




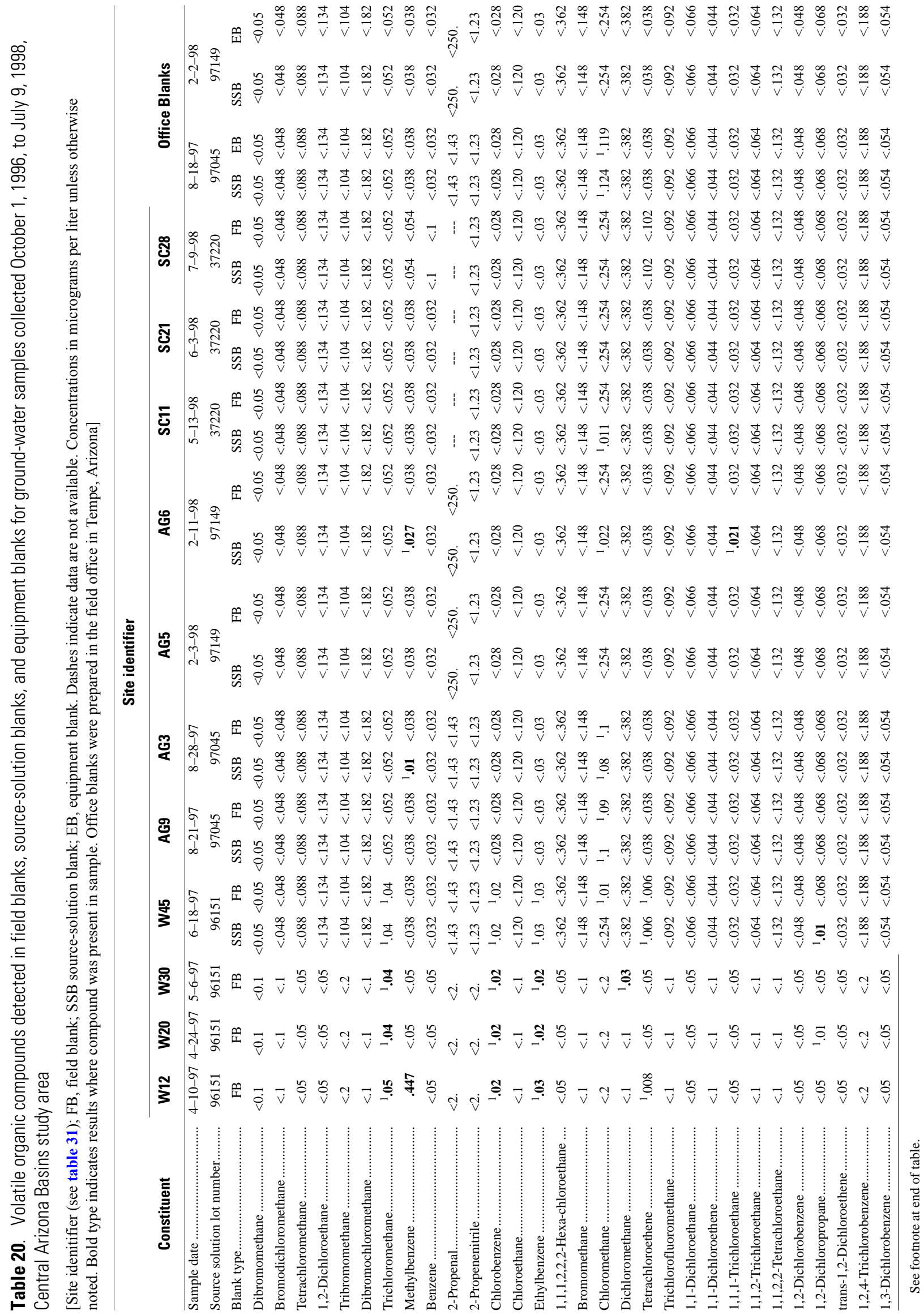




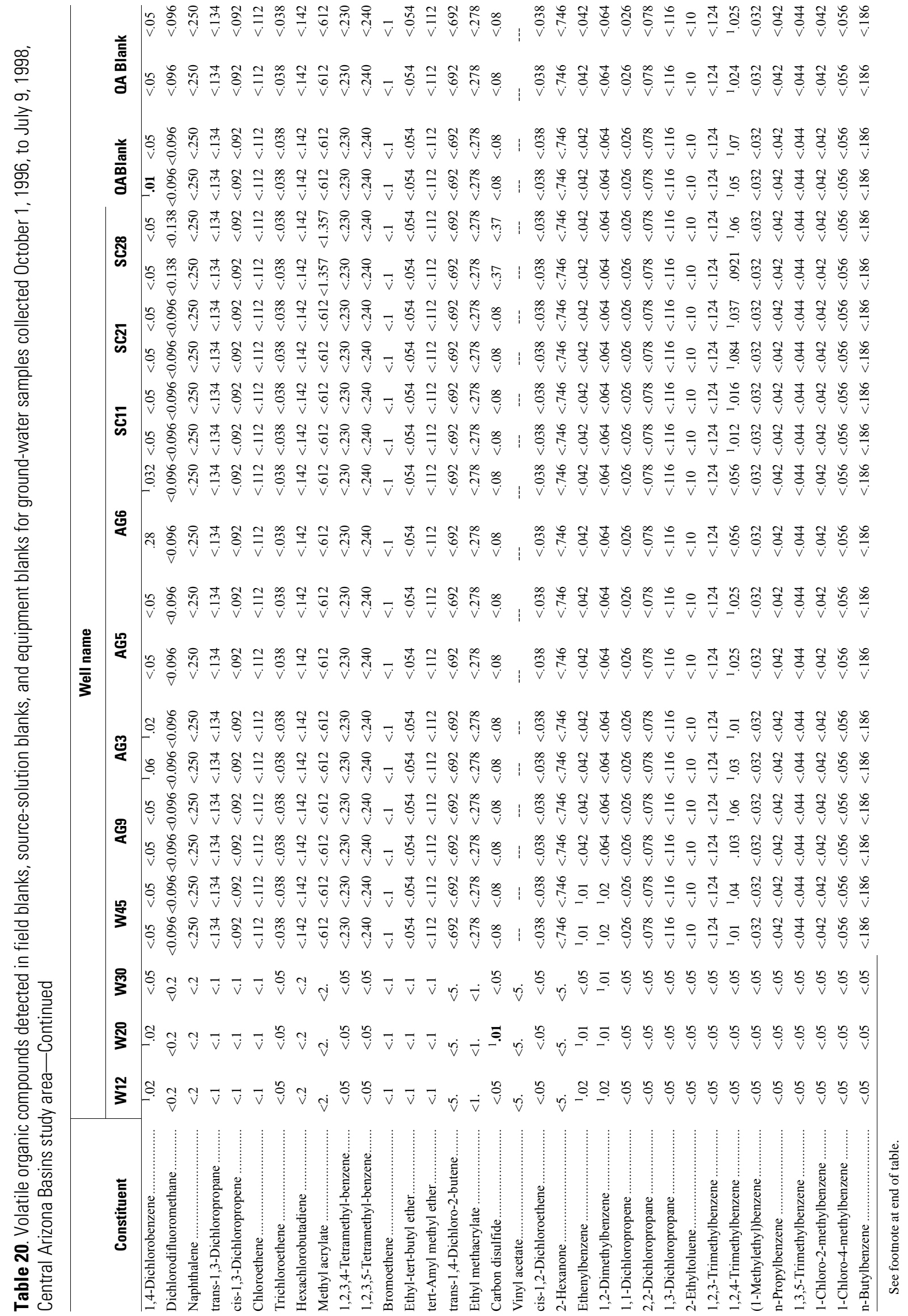




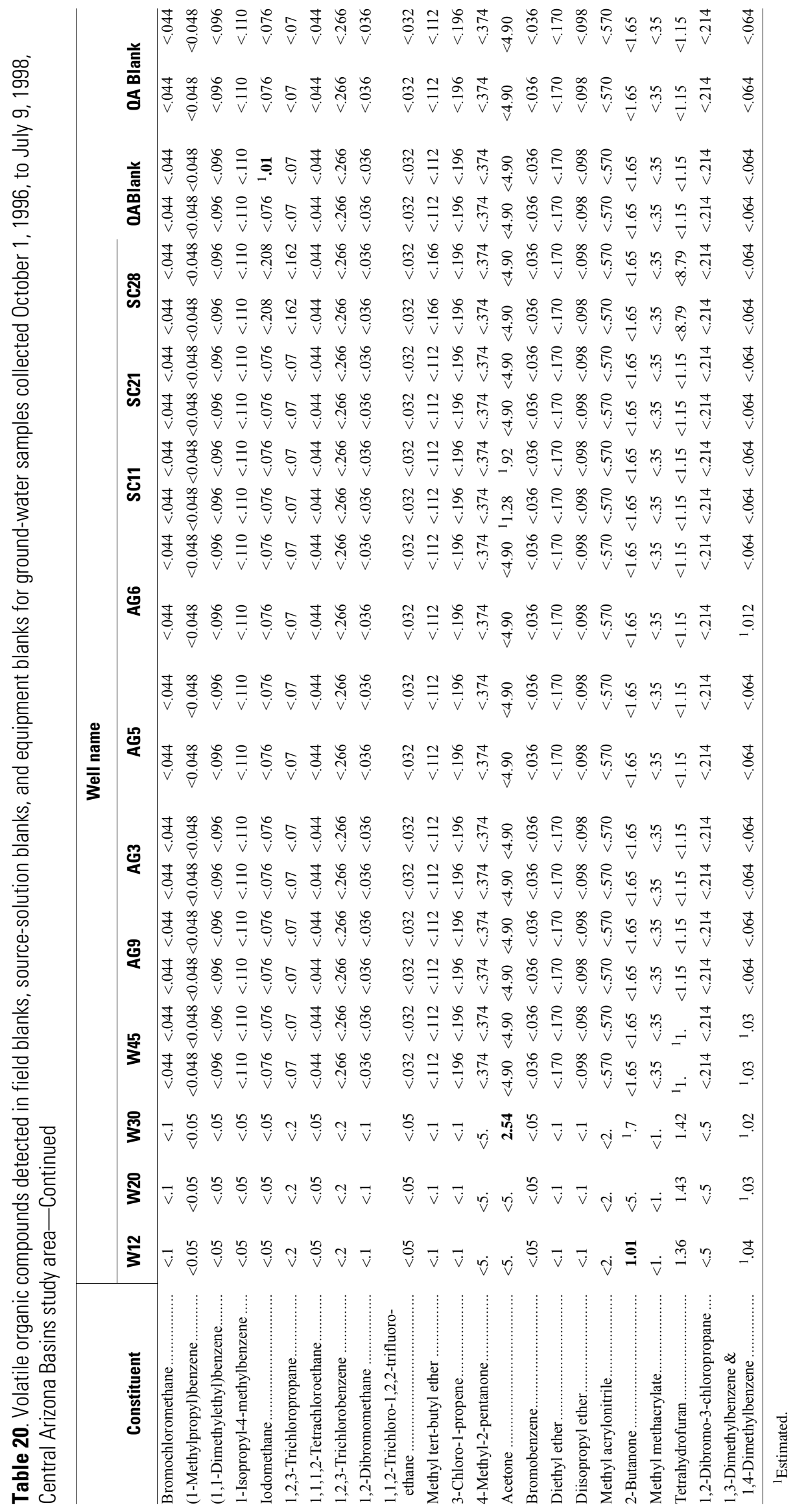

68 Characteristics of Shallow Deposits Beneath Rillito Creek, Pima County, Arizona 
Table 21. Volatile organic compounds detected in trip blanks and polyvinyl chloride wash samples for ground-water samples, Central Arizona Basins study area, 1996-98

[Site identifier (see table 31); TB, Trip blank; PVC, polyvinyl-chloride wash. Concentrations are in micrograms per liter unless otherwise indicated. Dashes indicate data are not available. Bold type indicates results where the compound was present in sample.]

\begin{tabular}{|c|c|c|c|c|c|}
\hline \multirow[b]{2}{*}{ Constituent } & \multicolumn{5}{|c|}{ Site identifier } \\
\hline & SV13 & W34 & SC11 & & \\
\hline Sample date & $8-14-96$ & $5-5-97$ & $5-13-98$ & & \\
\hline Blank type & $\mathrm{TB}$ & TB & $\mathrm{TB}$ & PVC & PVC \\
\hline Dibromomethane & $<0.1$ & $<0.1$ & $<0.05$ & ${ }^{1} 0.01$ & 0.13 \\
\hline Bromodichloromethane & $<.1$ & $<.1$ & $<.048$ & ${ }^{1} .01$ & ${ }^{1} .01$ \\
\hline Tetrachloromethane & $<.05$ & $<.05$ & $<.088$ & $<.088$ & $<.088$ \\
\hline 1,2-Dichloroethane & $<.05$ & $<.05$ & $<.134$ & $<.134$ & $<.134$ \\
\hline Tribromomethane & $<.2$ & $<.2$ & $<.104$ & 1.31 & ${ }^{1} .04$ \\
\hline Dibromochloromethane & $<.1$ & $<.1$ & $<.182$ & ${ }^{1} .06$ & $<.182$ \\
\hline Trichloromethane & $<.05$ & $<.05$ & $<.052$ & ${ }^{1} .05$ & ${ }^{1} .09$ \\
\hline Methylbenzene & $<.05$ & $<.05$ & ${ }^{1} .029$ & ${ }^{1} .06$ & $<.038$ \\
\hline Benzene & $<.05$ & $<.05$ & $<.032$ & ${ }^{1} .03$ & ${ }^{1} .02$ \\
\hline 2-Propenal & $<2$ & $<2$ & --- & $<1.43$ & $<1.43$ \\
\hline 2-Propenenitrile & $<2$ & $<2$ & $<1.23$ & $<1.23$ & $<1.23$ \\
\hline Chlorobenzene & $<.05$ & $<.05$ & $<.028$ & $<.028$ & $<.028$ \\
\hline Chloroethane & $<.1$ & $<.1$ & $<.120$ & $<.120$ & ${ }^{1} .03$ \\
\hline Ethylbenzene & $<.05$ & $<.05$ & $<.03$ & ${ }^{1} .01$ & $<.03$ \\
\hline 1,1,1,2,2,2-Hexa-chloroethane & $<.05$ & $<.05$ & $<.362$ & $<.362$ & $<.362$ \\
\hline Bromomethane & $<.1$ & $<.1$ & $<.148$ & $<.148$ & $<.148$ \\
\hline Chloromethane & $<.2$ & $<.2$ & $<.254$ & $<.254$ & 1.2 \\
\hline Dichloromethane & $<.1$ & $<.1$ & $<.382$ & $<.382$ & $<.382$ \\
\hline Tetrachloroethene & $<.05$ & $<.05$ & $<.038$ & $<.038$ & $<.038$ \\
\hline Trichlorofluoromethane & $<.1$ & $<.1$ & $<.092$ & $<.092$ & $<.092$ \\
\hline 1,1-Dichloroethane & $<.05$ & $<.05$ & $<.066$ & $<.066$ & $<.066$ \\
\hline 1,1-Dichloroethene & $<.1$ & $<.1$ & $<.044$ & $<.044$ & $<.044$ \\
\hline 1,1,1-Trichloroethane & $<.05$ & $<.05$ & $<.032$ & $<.032$ & $<.032$ \\
\hline 1,1,2-Trichloroethane & $<.1$ & $<.1$ & $<.064$ & $<.064$ & $<.064$ \\
\hline 1,1,2,2-Tetrachloroethane & $<.1$ & $<.1$ & $<.132$ & $<.132$ & $<.132$ \\
\hline 1,2-Dichlorobenzene & $<.05$ & $<.05$ & $<.048$ & $<.048$ & $<.048$ \\
\hline 1,2-Dichloropropane & $<.05$ & $<.05$ & $<.068$ & $<.068$ & $<.068$ \\
\hline trans-1,2-Dichloroethene & $<.05$ & $<.05$ & $<.032$ & $<.032$ & $<.032$ \\
\hline 1,2,4-Trichlorobenzene & $<.2$ & $<.2$ & $<.188$ & $<.188$ & $<.188$ \\
\hline 1,3-Dichlorobenzene & $<.05$ & $<.05$ & $<.054$ & $<.054$ & $<.054$ \\
\hline 1,4-Dichlorobenzene & ${ }^{1} .07$ & $<.05$ & $<.05$ & $<.05$ & $<.05$ \\
\hline Dichlorodifluoromethane & $<.2$ & $<.2$ & $<.096$ & $<.096$ & $<.096$ \\
\hline Naphthalene & $<.2$ & $<.2$ & $<.250$ & $<.250$ & ${ }^{1} .1$ \\
\hline trans-1,3-Dichloropropane & $<.1$ & $<.1$ & $<.134$ & $<.134$ & $<.134$ \\
\hline cis-1,3-Dichloropropene & $<.1$ & $<.1$ & $<.092$ & $<.092$ & $<.092$ \\
\hline Chloroethene & $<.1$ & $<.1$ & $<.112$ & $<.112$ & $<.112$ \\
\hline Trichloroethene & $<.05$ & $<.05$ & $<.038$ & $<.038$ & $<.038$ \\
\hline Hexachlorobutadiene & $<.2$ & $<.2$ & $<.142$ & $<.142$ & $<.142$ \\
\hline Methyl acrylate & $<2$ & $<2$ & $<.612$ & $<.612$ & $<.612$ \\
\hline 1,2,3,4-Tetramethylbenzene & $<.05$ & $<.05$ & $<.230$ & $<.230$ & ${ }^{1} .01$ \\
\hline 1,2,3,5-Tetramethylbenzene & $<.05$ & $<.05$ & $<.240$ & $<.240$ & ${ }^{1} .03$ \\
\hline Bromoethene & $<.1$ & $<.1$ & $<.1$ & $<.1$ & $<.1$ \\
\hline Ethyl-tert-butyl ether & $<.1$ & $<.1$ & $<.054$ & $<.054$ & $<.054$ \\
\hline
\end{tabular}


Table 21. Volatile organic compounds detected in trip blanks and polyvinyl chloride wash samples for ground-water samples, Central Arizona Basins study area, 1996-98-Continued

\begin{tabular}{|c|c|c|c|c|c|}
\hline \multirow[b]{2}{*}{ Constituent } & \multicolumn{5}{|c|}{ Site identifier } \\
\hline & SV13 & W34 & SC11 & & \\
\hline tert-Amyl methyl ether & $<0.1$ & $<0.1$ & $<0.112$ & $<0.112$ & $<0.112$ \\
\hline trans-1,4-Dichloro-2-butene & $<5$ & $<5$ & $<.692$ & $<.692$ & $<.692$ \\
\hline Ethyl methacrylate & $<1$. & $<1$. & $<.278$ & $<.278$ & $<.278$ \\
\hline Carbon disulfide & $<.05$ & $<.05$ & $<.08$ & ${ }^{1} .02$ & $<.08$ \\
\hline Vinyl acetate & $<5$ & $<5$ & --- & --- & --- \\
\hline cis-1,2-Dichloroethene & $<.05$ & $<.05$ & $<.038$ & $<.038$ & $<.038$ \\
\hline 2-Hexanone & $<5$ & $<5$. & $<.746$ & $<.746$ & $<.746$ \\
\hline Ethenylbenzene & $<.05$ & $<.05$ & $<.042$ & $<.042$ & $<.042$ \\
\hline 1,2-Dimethylbenzene & $<.05$ & $<.05$ & $<.064$ & ${ }^{1} .02$ & ${ }^{1} .01$ \\
\hline 1,1-Dichloropropene & $<.05$ & $<.05$ & $<.026$ & $<.026$ & $<.026$ \\
\hline 2,2-Dichloropropane & $<.05$ & $<.05$ & $<.078$ & $<.078$ & $<.078$ \\
\hline 1,3-Dichloropropane & $<.05$ & $<.05$ & $<.116$ & $<.116$ & $<.116$ \\
\hline 2-Ethyltoluene & $<.05$ & $<.05$ & $<.1$ & $<.1$ & ${ }^{1} .05$ \\
\hline 1,2,3-Trimethylbenzene & $<.05$ & $<.05$ & $<.124$ & $<.124$ & ${ }^{1} .03$ \\
\hline 1,2,4-Trimethylbenzene & $<.05$ & $<.05$ & $<.056$ & ${ }^{1} .02$ & .185 \\
\hline (1-Methylethyl)benzene & $<.05$ & $<.05$ & $<.032$ & $<.032$ & $<.032$ \\
\hline n-Propylbenzene & $<.05$ & $<.05$ & $<.042$ & $<.042$ & ${ }^{1} .02$ \\
\hline 1,3,5-Trimethylbenzene & $<.05$ & $<.05$ & $<.044$ & $<.044$ & ${ }^{1} .02$ \\
\hline 1-Chloro-2-methylbenzene & $<.05$ & $<.05$ & $<.042$ & $<.042$ & $<.042$ \\
\hline 1-Chloro-4-methylbenzene & $<.05$ & $<.05$ & $<.056$ & $<.056$ & $<.056$ \\
\hline Bromochloromethane & $<.1$ & $<.1$ & $<.044$ & $<.044$ & $<.044$ \\
\hline n-Butylbenzene & $<.05$ & $<.05$ & $<.186$ & $<.186$ & $<.186$ \\
\hline (1-Methylpropyl)benzene & $<.05$ & $<.05$ & $<.048$ & $<.048$ & $<.048$ \\
\hline (1,1-Dimethylethyl)benzene & $<.05$ & $<.05$ & $<.096$ & $<.096$ & $<.096$ \\
\hline 1-Isopropyl-4-methylbenzene & $<.05$ & $<.05$ & $<.110$ & ${ }^{1} .008$ & $<.11$ \\
\hline Iodomethane & $<.05$ & $<.05$ & $<.076$ & $<.076$ & ${ }^{1} .1$ \\
\hline 1,2,3-Trichloropropane & $<.2$ & $<.2$ & $<.07$ & $<.07$ & $<.07$ \\
\hline 1,1,1,2-Tetrachloroethane & $<.05$ & $<.05$ & $<.044$ & $<.044$ & $<.044$ \\
\hline 1,2,3-Trichlorobenzene & $<.2$ & $<.2$ & $<.266$ & $<.266$ & $<.266$ \\
\hline 1,2-Dibromomethane & $<.1$ & $<.1$ & $<.036$ & $<.036$ & $<.036$ \\
\hline 1,1,2-Trichloro-1,2,2-trifluoroethane & $<.05$ & $<.05$ & $<.032$ & $<.032$ & $<.032$ \\
\hline Methyl tert-butyl ether & $<.1$ & $<.1$ & $<.112$ & ${ }^{1} .08$ & ${ }^{1} .04$ \\
\hline 3-Chloro-1-propene & $<.1$ & $<.1$ & $<.196$ & $<.196$ & $<.196$ \\
\hline 4-Methyl-2-pentanone & $<5$ & $<5$ & $<.374$ & $<.374$ & 2.12 \\
\hline Acetone & $<5$ & $<5$. & $<4.90$ & ${ }^{1} 11$. & 124. \\
\hline Bromobenzene & $<.05$ & $<.05$ & $<.036$ & $<.036$ & $<.036$ \\
\hline Diethyl ether & $<.1$ & $<.1$ & $<.170$ & $<.170$ & $<.170$ \\
\hline Diisopropyl ether & $<.1$ & $<.1$ & $<.098$ & $<.098$ & $<.098$ \\
\hline Methyl acrylonitrile & $<2$ & $<2$ & $<.570$ & $<.570$ & $<.570$ \\
\hline 2-Butanone & $<5$ & $<5$. & $<1.65$ & $<1.65$ & $<1.65$ \\
\hline Methyl methacrylate & $<1$. & $<1$ & $<.35$ & $<.35$ & $<.35$ \\
\hline Tetrahydrofuran & $<5$ & $<5$. & $<1.15$ & $<1.15$ & $<1.15$ \\
\hline 1,2-Dibromo-3-chloropropane & $<.5$ & $<.5$ & $<.214$ & $<.214$ & $<.214$ \\
\hline $\begin{array}{l}\text { 1,3-Dimethylbenzene and } \\
\text { 1,4-Dimethylbenzene }\end{array}$ & $<.05$ & $<.05$ & $<.064$ & ${ }^{1} .05$ & $<.064$ \\
\hline
\end{tabular}


Table 22. Percent recovery statistics for pesticides and pesticide-degradation compounds detected in field-matrix and laboratory-spike samples for ground-water samples and in laboratory set-spike samples, Central Arizona Basins study area, 1996-98

[Concentrations are in micrograms per liter unless otherwise indicated. Dashes indicate no data available. Compounds in bold font may be affected by matrix effects; compounds shaded may be affected by field techniques; n, number of samples; IQR, interquartile range; FMS-LS, field-matrix spike sample median minus the laboratory-spike sample median; FMS-SS, field-matrix spike median minus the set-spike median; GCMS, gas chromatography/mass spectrometry; HPLC, high-performance liquid chromatography; WATSTORE, National Water Data Storage Retrieval System]

\begin{tabular}{|c|c|c|c|c|c|c|c|c|c|c|c|c|c|c|}
\hline \multirow[b]{3}{*}{ 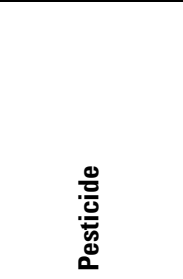 } & \multirow{3}{*}{ 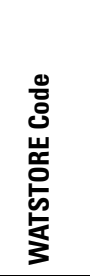 } & \multirow{3}{*}{ 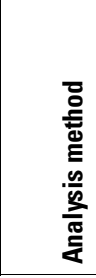 } & \multicolumn{12}{|c|}{ Percent recovery } \\
\hline & & & \multicolumn{4}{|c|}{$\begin{array}{c}\text { Field-matrix } \\
\text { spike samples }\end{array}$} & \multicolumn{4}{|c|}{$\begin{array}{c}\text { Laboratory-spike } \\
\text { samples }\end{array}$} & \multicolumn{2}{|c|}{ Set spikes ${ }^{1}$} & \multicolumn{2}{|c|}{$\begin{array}{c}\text { Relative } \\
\text { difference }\end{array}$} \\
\hline & & & $=$ & 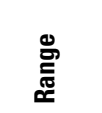 & 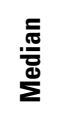 & 뜨므 & $=$ & 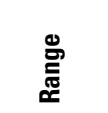 & 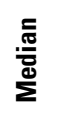 & 똠 & 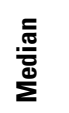 & 뜸 & $\begin{array}{l}\text { es } \\
\sum_{i}^{\infty}\end{array}$ & $\begin{array}{l}\mathscr{P} \\
\sum_{i}^{\infty}\end{array}$ \\
\hline Acetochlor & 49260 & GCMS & 21 & $81-120$ & 109 & 8 & 4 & 84-109 & 104 & 6 & 102 & 12 & 5 & 12 \\
\hline Acifluorfen & 49315 & HPLC & 22 & $57-117$ & 87 & 12 & 3 & $85-118$ & 94 & 17 & 88 & 11 & 7 & 1 \\
\hline Alachlor & 46342 & GCMS & 21 & $87-118$ & 109 & 7 & 4 & $93-113$ & 106 & 14 & 102 & 11 & 3 & 7 \\
\hline Aldicarb $^{2}$ & 49312 & HPLC & 16 & $30-94$ & 61 & 32 & 3 & $29-84$ & 77 & 28 & 43 & 28 & 21 & 41 \\
\hline $\begin{array}{l}\text { Aldicarb } \\
\text { sulfone }^{2}\end{array}$ & 49313 & HPLC & 22 & $0-51$ & 14 & 33 & 3 & $49-76$ & 58 & 14 & 60 & 16 & 76 & 77 \\
\hline $\begin{array}{l}\text { Aldicarb } \\
\quad \text { sulfoxide }^{2}\end{array}$ & 49314 & HPLC & 22 & $0-103$ & 60 & 46 & 3 & $63-150$ & 120 & 43 & 90 & 25 & 50 & 33 \\
\hline Atrazine & 39632 & GCMS & 21 & $80-118$ & 102 & 13 & 4 & $89-112$ & 104 & 8 & 100 & 11 & 3 & 2 \\
\hline Azinphos-methyl & 82686 & GCMS & 21 & $43-729$ & 100 & 59 & 4 & $48-113$ & 99 & 18 & 80 & 56 & 1 & 25 \\
\hline Benfluralin & 82673 & GCMS & 21 & $36-102$ & 78 & 32 & 4 & $72-94$ & 83 & 21 & 67 & 21 & 6 & 16 \\
\hline Bentazon & 38711 & HPLC & 20 & 60-94 & 78 & 12 & 3 & $73-92$ & 76 & 9 & 80 & 13 & 3 & 3 \\
\hline Bromacil & 04029 & HPLC & 22 & $65-102$ & 85 & 20 & 3 & $71-99$ & 90 & 14 & 82 & 15 & 6 & 3 \\
\hline Bromoxynil & 49311 & HPLC & 22 & $12-101$ & 84 & 10 & 3 & $82-87$ & 82 & 2 & 85 & 11 & 3 & 1 \\
\hline Butylate & 04028 & GCMS & 21 & $86-121$ & 101 & 9 & 4 & 85-104 & 95 & 11 & 89 & 9 & 6 & 13 \\
\hline Carbaryl $^{2}$ & 82680 & GCMS & 21 & $51-491$ & 158 & 138 & 4 & $106-207$ & 129 & 36 & 126 & 74 & 23 & 26 \\
\hline Carbaryl & 49310 & HPLC & 20 & $47-97$ & 79 & 8 & 3 & $72-94$ & 80 & 11 & 88 & 12 & 2 & 11 \\
\hline Carbofuran $^{2}$ & 82674 & GCMS & 21 & $68-267$ & 130 & 76 & 4 & $103-170$ & 130 & 21 & 113 & 37 & 0 & 15 \\
\hline Carbofuran & 49309 & HPLC & 22 & $69-121$ & 82 & 21 & 3 & $67-110$ & 99 & 22 & 83 & 15 & 17 & 1 \\
\hline Chloramben & 49307 & HPLC & 16 & $0-95$ & 47 & 70 & 1 & 88 & --- & --- & 70 & 18 & --- & 33 \\
\hline $\begin{array}{l}\text { Chloro- } \\
\text { thalonil }^{2}\end{array}$ & 49306 & HPLC & 20 & $0-58$ & 25 & 20 & 3 & $19-87$ & 56 & 34 & 36 & 20 & 54 & 30 \\
\hline Chlorpyrifos & 38933 & GCMS & 21 & 68-107 & 93 & 23 & 4 & 82-104 & 84 & 7 & 90 & 12 & 11 & 4 \\
\hline Clopyralid & 49305 & HPLC & 21 & $0-68$ & 25 & 61 & 3 & $67-81$ & 77 & 7 & 77 & 19 & 67 & 67 \\
\hline Cyanazine & 04041 & GCMS & 21 & $28-132$ & 107 & 24 & 4 & $37-123$ & 109 & 31 & 103 & 25 & 1 & 4 \\
\hline 2,4-D & 39732 & HPLC & 20 & 46-99 & 82 & 9 & 3 & $67-89$ & 79 & 11 & 86 & 12 & 4 & 4 \\
\hline Dacthal & 82682 & GCMS & 21 & $91-125$ & 110 & 20 & 4 & $92-127$ & 113 & 9 & 103 & 13 & 3 & 7 \\
\hline $\begin{array}{l}\text { Dacthal mono- } \\
\quad \text { acid }\end{array}$ & 49304 & HPLC & 22 & $60-107$ & 88 & 5 & 3 & $85-90$ & 86 & 2 & 83 & 11 & 2 & 6 \\
\hline 2,4-DB & 38746 & HPLC & 22 & $8-121$ & 74 & 14 & 3 & $71-83$ & 73 & 6 & 81 & 16 & 2 & 8 \\
\hline p,p'-DDE & 34653 & GCMS & 21 & $50-119$ & 75 & 23 & 4 & $51-103$ & 55 & 17 & 58 & 13 & 37 & 29 \\
\hline $\begin{array}{l}\text { Deethyl- } \\
\quad \text { atrazine }^{2}\end{array}$ & 04040 & GCMS & 21 & $27-85$ & 56 & 23 & 4 & $36-79$ & 49 & 12 & 45 & 18 & 14 & 24 \\
\hline Diazinon & 39572 & GCMS & 21 & $62-105$ & 94 & 13 & 4 & $62-94$ & 87 & 13 & 88 & 12 & 8 & 7 \\
\hline Dicamba & 38442 & HPLC & 22 & $15-103$ & 73 & 28 & 3 & $68-86$ & 77 & 9 & 82 & 12 & 4 & 11 \\
\hline Dichlobenil $^{2}$ & 49303 & HPLC & 13 & 4-90 & 58 & 10 & 3 & $43-72$ & 63 & 14 & 48 & 38 & 8 & 21 \\
\hline
\end{tabular}

See footnotes at end of table. 
Table 22. Percent recovery statistics for pesticides and pesticide-degradation compounds detected in field-matrix and laboratory-spike samples for ground-water samples and in laboratory set-spike samples, Central Arizona Basins study area,

\begin{tabular}{|c|c|c|c|c|c|c|c|c|c|c|c|c|c|c|}
\hline \multirow[b]{3}{*}{ 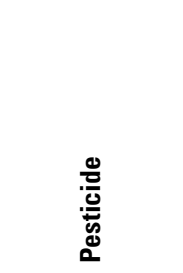 } & \multirow{3}{*}{ 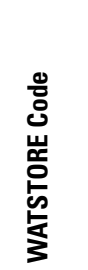 } & \multirow{3}{*}{ 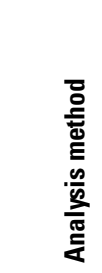 } & \multicolumn{12}{|c|}{ Percent recovery } \\
\hline & & & \multicolumn{4}{|c|}{$\begin{array}{c}\text { Field matrix } \\
\text { spike samples }\end{array}$} & \multicolumn{4}{|c|}{$\begin{array}{l}\text { Laboratory spike } \\
\text { samples }\end{array}$} & \multicolumn{2}{|c|}{ Set spikes ${ }^{1}$} & \multicolumn{2}{|c|}{$\begin{array}{c}\text { Relative } \\
\text { difference }\end{array}$} \\
\hline & & & $=$ & 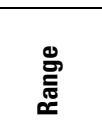 & .: & 똠 & $=$ & 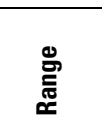 & 挋 & 똥 & 挋 & 똠 & $\sum_{i}^{\infty}$ & $\sum_{\substack{1 \\
4}}^{\infty}$ \\
\hline Dichlorprop & 49302 & HPLC & 22 & $54-100$ & 81 & 12 & 3 & $86-95$ & 92 & 4 & 85 & 12 & 12 & 5 \\
\hline Dieldrin & 39381 & GCMS & 21 & $82-128$ & 101 & 11 & 4 & 74-91 & 86 & 9 & 81 & 16 & 17 & 24 \\
\hline $\begin{array}{l}\text { 2,6-Diethyl- } \\
\text { aniline }\end{array}$ & 82660 & GCMS & 21 & $67-106$ & 93 & 11 & 4 & 84-104 & 90 & 7 & 85 & 9 & 3 & 9 \\
\hline Dinoseb & 49301 & HPLC & 20 & $63-95$ & 82 & 7 & 3 & $81-83$ & 82 & 1 & 81 & 14 & 0 & 2 \\
\hline Disulfoton & 82677 & GCMS & 21 & $37-112$ & 81 & 29 & 4 & $78-104$ & 86 & 11 & 73 & 23 & 6 & 11 \\
\hline Diuron & 49300 & HPLC & 13 & $31-130$ & 85 & 10 & 3 & 79-99 & 80 & 10 & 88 & 13 & 6 & 4 \\
\hline DNOC $^{2}$ & 49299 & HPLC & 22 & $0-73$ & 52 & 19 & 3 & $19-56$ & 50 & 18 & 39 & 19 & 4 & 34 \\
\hline EPTC & 82668 & GCMS & 21 & $70-107$ & 94 & 16 & 4 & $78-105$ & 88 & 12 & 90 & 9 & 7 & 4 \\
\hline Ethalfluralin & 82663 & GCMS & 21 & $52-133$ & 93 & 38 & 4 & $87-123$ & 105 & 33 & 78 & 23 & 12 & 19 \\
\hline Ethoprop & 82672 & GCMS & 21 & $63-119$ & 102 & 12 & 4 & 73-106 & 97 & 18 & 95 & 17 & 5 & 7 \\
\hline Fenuron & 49297 & HPLC & 22 & $68-94$ & 84 & 13 & 3 & $80-103$ & 84 & 12 & 89 & 11 & 0 & 5 \\
\hline Fluometuron & 38811 & HPLC & 22 & 73-109 & 87 & 11 & 3 & 79-101 & 92 & 11 & 93 & 18 & 5 & 6 \\
\hline Fonofos & 04095 & GCMS & 21 & $63-103$ & 92 & 6 & 4 & 89-96 & 94 & 3 & 88 & 13 & 1 & 5 \\
\hline alpha-HCH & 34253 & GCMS & 12 & $82-121$ & 102 & 13 & 1 & 83 & --- & --- & 90 & 13 & --- & 13 \\
\hline gamma-HCH & 39341 & GCMS & 21 & $81-120$ & 99 & 19 & 4 & $82-108$ & 90 & 13 & 92 & 15 & 10 & 8 \\
\hline $\begin{array}{l}\text { 3-Hydroxy- } \\
\text { carbo-furan }\end{array}$ & 49308 & HPLC & 22 & 49-106 & 74 & 21 & 3 & $59-111$ & 78 & 26 & 76 & 13 & 6 & 3 \\
\hline Linuron & 82666 & GCMS & 21 & $82-221$ & 116 & 41 & 4 & $67-118$ & 98 & 22 & 101 & 29 & 18 & 14 \\
\hline Linuron & 38478 & HPLC & 20 & 58-99 & 80 & 12 & 3 & $78-105$ & 95 & 14 & 85 & 10 & 16 & 6 \\
\hline МCPA & 38482 & HPLC & 22 & 37-99 & 70 & 22 & 3 & $55-71$ & 66 & 8 & 84 & 13 & 7 & 17 \\
\hline МСРВ & 38487 & HPLC & 22 & $21-93$ & 69 & 17 & 3 & $68-78$ & 75 & 5 & 77 & 15 & 9 & 11 \\
\hline Malathion & 39532 & GCMS & 21 & $71-111$ & 99 & 18 & 4 & $87-123$ & 98 & 16 & 95 & 22 & 1 & 4 \\
\hline Methiocarb & 38501 & HPLC & 22 & $47-93$ & 75 & 16 & 3 & $71-90$ & 77 & 9 & 85 & 14 & 3 & 12 \\
\hline Methomyl & 49296 & HPLC & 22 & 64-99 & 88 & 18 & 3 & $71-103$ & 103 & 16 & 90 & 16 & 15 & 3 \\
\hline Methyl parathion & 82667 & GCMS & 21 & $50-240$ & 99 & 60 & 4 & $86-132$ & 103 & 12 & 89 & 27 & 4 & 11 \\
\hline Metolachlor & 39415 & GCMS & 21 & $95-166$ & 120 & 10 & 4 & $80-123$ & 110 & 22 & 106 & 16 & 9 & 13 \\
\hline Metribuzin & 82630 & GCMS & 21 & $69-111$ & 88 & 25 & 4 & $78-128$ & 95 & 17 & 78 & 18 & 7 & 13 \\
\hline Molinate & 82671 & GCMS & 21 & $70-107$ & 95 & 10 & 4 & $88-103$ & 97 & 7 & 94 & 8 & 2 & 1 \\
\hline Napropamide & 82684 & GCMS & 21 & $86-127$ & 97 & 11 & 4 & $85-98$ & 94 & 3 & 94 & 15 & 3 & 3 \\
\hline Neburon & 49294 & HPLC & 22 & $0-98$ & 72 & 25 & 3 & $76-105$ & 77 & 15 & 85 & 13 & 7 & 16 \\
\hline Norflurazon & 49293 & HPLC & 20 & $72-112$ & 86 & 25 & 3 & 79-101 & 98 & 11 & 88 & 12 & 12 & 2 \\
\hline Oryzalin & 49292 & HPLC & 18 & $21-77$ & 65 & 20 & 3 & $56-105$ & 105 & 25 & 75 & 22 & 38 & 13 \\
\hline Oxamyl & 38866 & HPLC & 22 & $29-76$ & 64 & 13 & 3 & $62-90$ & 66 & 14 & 77 & 13 & 4 & 17 \\
\hline Parathion & 39542 & GCMS & 21 & $49-127$ & 93 & 46 & 4 & $98-123$ & 105 & 17 & 93 & 22 & 12 & 0 \\
\hline Pebulate & 82669 & GCMS & 21 & $69-120$ & 99 & 15 & 4 & $87-105$ & 92 & 5 & 90 & 8 & 8 & 8 \\
\hline Pendimeth-alin & 82683 & GCMS & 21 & $35-120$ & 93 & 32 & 4 & $85-94$ & 92 & 4 & 73 & 24 & 0 & 27 \\
\hline $\begin{array}{l}\text { cis-Per- } \\
\text { methrin }\end{array}$ & 82687 & GCMS & 21 & $29-115$ & 60 & 15 & 4 & $30-66$ & 63 & 14 & 45 & 19 & 4 & 34 \\
\hline
\end{tabular}


Table 22. Percent recovery statistics for pesticides and pesticide-degradation compounds detected in field-matrix and laboratory-spike samples for ground-water samples and in laboratory set-spike samples, Central Arizona Basins study area,

1996-98-Continued

\begin{tabular}{|c|c|c|c|c|c|c|c|c|c|c|c|c|c|c|}
\hline \multirow[b]{3}{*}{ 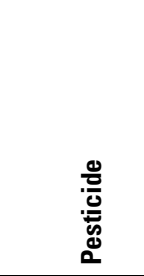 } & \multirow{3}{*}{ 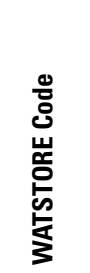 } & \multirow{3}{*}{ 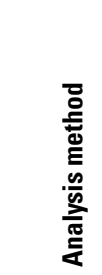 } & \multicolumn{12}{|c|}{ Percent recovery } \\
\hline & & & \multicolumn{4}{|c|}{$\begin{array}{c}\text { Field matrix } \\
\text { spike samples }\end{array}$} & \multicolumn{4}{|c|}{$\begin{array}{l}\text { Laboratory spike } \\
\text { samples }\end{array}$} & \multicolumn{2}{|c|}{ Set spikes ${ }^{1}$} & \multicolumn{2}{|c|}{$\begin{array}{r}\text { Relative } \\
\text { difference }\end{array}$} \\
\hline & & & $=$ & 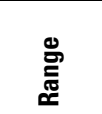 & 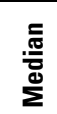 & 똠 & $=$ & 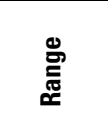 & 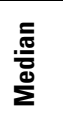 & 뜸 & 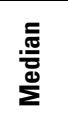 & 뜸 & $\sum_{i}^{\infty}$ & p \\
\hline Phorate & 82664 & GCMS & 21 & $41-112$ & 82 & 22 & 4 & $75-94$ & 83 & 13 & 72 & 19 & 0 & 14 \\
\hline Picloram & 49291 & HPLC & 21 & $17-93$ & 76 & 30 & 3 & $41-90$ & 80 & 25 & 81 & 13 & 4 & 6 \\
\hline Prometon & 04037 & GCMS & 21 & $74-129$ & 103 & 20 & 4 & $88-107$ & 92 & 12 & 71 & 43 & 11 & 45 \\
\hline Pronamide & 82676 & GCMS & 12 & 69-109 & 91 & 26 & 1 & 106 & --- & --- & 89 & 14 & --- & 2 \\
\hline Propachlor & 04024 & GCMS & 21 & $95-113$ & 105 & 7 & 4 & $93-138$ & 112 & 27 & 105 & 14 & 6 & 0 \\
\hline Propanil & 82679 & GCMS & 21 & $74-123$ & 109 & 15 & 4 & $103-113$ & 112 & 4 & 108 & 17 & 3 & 1 \\
\hline Propargite & 82685 & GCMS & 21 & $49-145$ & 88 & 33 & 4 & 61-109 & 68 & 21 & 71 & 23 & 30 & 24 \\
\hline Propham & 49236 & HPLC & 17 & $50-96$ & 62 & 13 & 2 & $67-80$ & 73 & 7 & 68 & 21 & 16 & 9 \\
\hline Propoxur & 38538 & HPLC & 22 & $60-230$ & 78 & 21 & 3 & 64-91 & 88 & 14 & 73 & 11 & 11 & 7 \\
\hline Silvex & 39762 & HPLC & 22 & $35-96$ & 84 & 13 & 3 & $83-86$ & 84 & 2 & 88 & 13 & 0 & 4 \\
\hline Simazine & 04035 & GCMS & 21 & $77-119$ & 103 & 8 & 4 & $93-111$ & 102 & 8 & 101 & 13 & 1 & 2 \\
\hline $2,4,5-T$ & 39742 & HPLC & 16 & $84-127$ & 101 & 26 & 3 & $81-89$ & 82 & 4 & 82 & 11 & 22 & 23 \\
\hline Tebuthiuron & 82670 & GCMS & 21 & $75-141$ & 103 & 45 & 4 & $129-163$ & 136 & 17 & 116 & 39 & 24 & 11 \\
\hline Terbacil $^{2}$ & 82665 & GCMS & 21 & $58-120$ & 89 & 17 & 4 & $78-116$ & 98 & 18 & 80 & 29 & 9 & 11 \\
\hline Terbufos & 82675 & GCMS & 21 & $54-110$ & 92 & 13 & 4 & $83-90$ & 84 & 3 & 76 & 18 & 9 & 21 \\
\hline Thiobencarb & 82681 & GCMS & 21 & $72-120$ & 107 & 11 & 4 & $96-113$ & 103 & 4 & 100 & 15 & 4 & 7 \\
\hline Triallate & 82678 & GCMS & 21 & $64-122$ & 94 & 13 & 4 & $87-104$ & 92 & 6 & 89 & 14 & 2 & 5 \\
\hline Triclopyr & 49235 & HPLC & 20 & $47-98$ & 77 & 25 & 3 & $43-72$ & 53 & 14 & 79 & 12 & 46 & 2 \\
\hline Trifluralin & 82661 & GCMS & 21 & $37-111$ & 77 & 33 & 4 & 75-104 & 90 & 27 & 71 & 23 & 14 & 9 \\
\hline
\end{tabular}

${ }^{1}$ Source: J.D. Martin, hydrologist, U.S. Geological Survey, written commun., 1999.

${ }^{2}$ Analytical performance for this compound is considered poor (J.D. Martin, hydrologist, U.S. Geological Survey, written commun., 1999). 
Table 23. Percent recovery statistics for surrogate compounds for pesticides and volatile organic compounds detected in ground-water and surface-water samples, Central Arizona Basins study area, 1996-98

\begin{tabular}{|c|c|c|c|c|c|c|c|c|}
\hline \multirow[b]{2}{*}{ Compound name } & \multicolumn{4}{|c|}{$\begin{array}{l}\text { Percent recovery for environmental ground-water } \\
\text { samples }\end{array}$} & \multicolumn{4}{|c|}{$\begin{array}{l}\text { Percent recovery for environmental and replicate } \\
\text { surface-water samples }\end{array}$} \\
\hline & $\begin{array}{l}\text { Number of } \\
\text { samples }\end{array}$ & Minimum & Maximum & Median & $\begin{array}{l}\text { Number of } \\
\text { samples }\end{array}$ & Minimum & Maximum & Median \\
\hline \multicolumn{9}{|c|}{ Surrogate compounds for pesticides analyzed by gas chromatography/mass spectrometry } \\
\hline Terbuthylazine & 108 & 3 & 221 & 108 & 73 & 96 & 303 & 151 \\
\hline alpha-HCH-D6 & 108 & 3 & 220 & 100 & 73 & 69 & 154 & 99 \\
\hline Diazinon-D10 & 108 & 3 & 200 & 99 & 73 & 75 & 165 & 100 \\
\hline
\end{tabular}

Surrogate compound for pesticides analyzed by high-performance liquid chromatography

\begin{tabular}{lcccccccc}
\hline BDMC & 90 & 54 & 117 & 89.5 & 73 & 52 & 214 & 93 \\
\hline \multicolumn{7}{c}{ Surrogate compounds for volatile organic compounds } \\
\hline 1,2-Dichloroethane-d4 & 88 & 90 & 125 & 106 & 12 & 98 & 114 \\
Toluene-d8 & 88 & 94 & 111 & 99 & 12 & 98 & 101 & 99.5 \\
1,4-Bromoflurorbenzene & 88 & 79 & 122 & 95 & 12 & 97 & 105 & 100 \\
\hline
\end{tabular}


Table 24. Percent recovery statistics for volatile organic compounds detected in field-matrix spike samples for ground-water and surfacewater samples, Central Arizona Basins study area, 1996-98

[Dashes indicate no data available; n, number of samples; WATSTORE, National Water Data Storage Retrieval System]

\begin{tabular}{|c|c|c|c|c|c|c|c|}
\hline \multirow[b]{2}{*}{ Volatile organic compound } & \multirow[b]{2}{*}{$\begin{array}{l}\text { WATSTORE } \\
\text { code }\end{array}$} & \multicolumn{4}{|c|}{ Ground-water samples } & \multicolumn{2}{|c|}{ Surface-water sample } \\
\hline & & $\mathbf{n}$ & $\begin{array}{c}\text { Range of } \\
\text { recoveries, } \\
\text { in percent }\end{array}$ & $\begin{array}{l}\text { Median recovery, } \\
\text { in percent }\end{array}$ & $\begin{array}{l}\text { Interquartile } \\
\text { range }\end{array}$ & $\mathbf{n}$ & $\begin{array}{l}\text { Recovery, } \\
\text { in percent }\end{array}$ \\
\hline Dibromomethane & 30217 & 4 & $81-89$ & 85 & 7 & 0 & --- \\
\hline Bromodichloromethane & 32101 & 25 & $69-160$ & 96 & 30 & 1 & 86 \\
\hline Dibromochloromethane & 32105 & 25 & $67-137$ & 96 & 20 & 1 & 83 \\
\hline Trichloromethane & 32106 & 4 & $81-153$ & 118 & 69 & 0 & --- \\
\hline Methylbenzene & 34010 & 4 & $90-96$ & 90 & 2 & 0 & --- \\
\hline Chlorobenzene & 34301 & 4 & $83-88$ & 86.5 & 3 & 0 & --- \\
\hline Tetrachloroethene & 34475 & 25 & $44-192$ & 91 & 57 & 1 & 76 \\
\hline 1,4-Dichlorobenzene & 34571 & 25 & $54-153$ & 86 & 38 & 1 & 67 \\
\hline Carbon disulfide & 77041 & 4 & $72-88$ & 80 & 4 & 0 & --- \\
\hline Bromochloromethane & 77297 & 4 & $82-87$ & 84.5 & 4 & 0 & --- \\
\hline Tetrachloromethane & 32102 & 25 & $44-192$ & 87 & 39 & 1 & 63 \\
\hline 1,2-Dichloroethane & 32103 & 25 & $76-160$ & 100 & 26 & 1 & 71 \\
\hline Tribromomethane & 32104 & 25 & $75-137$ & 99 & 19 & 1 & 73 \\
\hline Benzene & 34030 & 4 & $84-88$ & 85.5 & 3 & 0 & --- \\
\hline 2-Propenal & 34210 & 4 & $35-41$ & 37.5 & 2 & 0 & --- \\
\hline 2-Propenenitrile & 34215 & 4 & $84-92$ & 87 & 7 & 0 & --- \\
\hline Chloroethane & 34311 & 4 & $66-82$ & 74.5 & 4 & 0 & --- \\
\hline Ethylbenzene & 34371 & 25 & $51-160$ & 86 & 39 & 1 & 68 \\
\hline 1,1,1,2,2,2-Hexachloroethane & 34396 & 4 & $86-89$ & 86.5 & 2 & 0 & --- \\
\hline Bromomethane & 34413 & 4 & $68-82$ & 74.5 & 10 & 0 & --- \\
\hline Chloromethane & 34418 & 4 & $38-73$ & 49.5 & 17 & 0 & --- \\
\hline Dichloromethane & 34423 & 4 & 94-102 & 100.5 & 3 & 0 & --- \\
\hline Trichlorofluoromethane & 34488 & 4 & $66-78$ & 73.5 & 4 & 0 & --- \\
\hline 1,1-Dichloroethane & 34496 & 4 & $84-90$ & 88 & 3 & 0 & --- \\
\hline 1,1-Dichloroethene & 34501 & 25 & 34-192 & 80 & 26 & 1 & 58 \\
\hline 1,1,1-Trichloroethane & 34506 & 25 & $51-176$ & 91 & 28 & 1 & 67 \\
\hline 1,1,2-Trichloroethane & 34511 & 4 & $86-95$ & 92.5 & 5 & 0 & --- \\
\hline 1,1,2,2-Tetrachloroethane & 34516 & 4 & 89-99 & 92.5 & 3 & 0 & --- \\
\hline 1,2-Dichlorobenzene & 34536 & 4 & $87-96$ & 88.5 & 3 & 0 & --- \\
\hline 1,2-Dichloropropane & 34541 & 4 & $82-88$ & 85 & 6 & 0 & --- \\
\hline trans-1,2-Dichloroethene & 34546 & 4 & $83-90$ & 87.5 & 3 & 0 & --- \\
\hline 1,2,4-Trichlorobenzene & 34551 & 4 & $78-84$ & 81 & 3 & 0 & --- \\
\hline 1,3-Dichlorobenzene & 34566 & 4 & $85-90$ & 86.5 & 4 & 0 & --- \\
\hline Dichlorodifluoromethane & 34668 & 4 & $36-97$ & 53.5 & 28 & 0 & --- \\
\hline Naphthalene & 34696 & 4 & $61-88$ & 74 & 26 & 0 & --- \\
\hline trans-1,3-Dichloropropene & 34699 & 4 & $80-90$ & 82.5 & 6 & 0 & --- \\
\hline cis-1,3-Dichloropropene & 34704 & 4 & $81-88$ & 84.5 & 4 & 0 & --- \\
\hline Chloroethene & 39175 & 25 & $0-223$ & 64 & 44 & 1 & 27 \\
\hline
\end{tabular}


Table 24. Percent recovery statistics for volatile organic compounds detected in field-matrix spike samples for ground-water and surfacewater samples, Central Arizona Basins study area, 1996-98-Continued

\begin{tabular}{|c|c|c|c|c|c|c|c|}
\hline \multirow[b]{2}{*}{ Volatile organic compound } & \multirow[b]{2}{*}{$\begin{array}{l}\text { WATSTORE } \\
\text { code }\end{array}$} & \multicolumn{4}{|c|}{ Ground-water samples } & \multicolumn{2}{|c|}{ Surface-water sample } \\
\hline & & $\mathbf{n}$ & $\begin{array}{l}\text { Range of } \\
\text { recoveries, in } \\
\text { percent }\end{array}$ & $\begin{array}{l}\text { Median } \\
\text { recovery, in } \\
\text { percent }\end{array}$ & $\begin{array}{l}\text { Inter- } \\
\text { quartile } \\
\text { range }\end{array}$ & $\mathbf{n}$ & $\begin{array}{c}\text { Recovery, in } \\
\text { percent }\end{array}$ \\
\hline Trichloroethene & 39180 & 25 & $52-192$ & 93 & 43 & 1 & 69 \\
\hline Hexachlorobutadiene & 39702 & 4 & $83-86$ & 84 & 1 & 0 & --- \\
\hline Methyl acrylate & 49991 & 4 & $79-91$ & 84 & 3 & 0 & --- \\
\hline 1,2,3,4-Tetramethylbenzene & 49999 & --- & --- & -- & --- & 0 & --- \\
\hline 1,2,3,5-Tetramethylbenzene & 50000 & 4 & $68-74$ & 71 & 3 & 0 & --- \\
\hline Bromoethene & 50002 & 4 & $72-84$ & 79 & 6 & 0 & --- \\
\hline Ethyl-tert-butyl ether & 50004 & 4 & $77-85$ & 81 & 7 & 0 & --- \\
\hline tert-Amyl methyl ether & 50005 & 4 & $77-82$ & 79.5 & 5 & 0 & --- \\
\hline trans-1,4-Dichloro-2-butene & 73547 & 4 & $78-88$ & 79 & 4 & 0 & -- \\
\hline Ethyl methacrylate & 73570 & 4 & $72-80$ & 75.5 & 6 & 0 & -- \\
\hline Vinyl acetate & 77057 & --- & --- & -- & --- & 0 & --- \\
\hline cis-1,2-Dichloroethene & 77093 & 4 & $82-88$ & 85.5 & 4 & 0 & --- \\
\hline 2-Hexanone & 77103 & 4 & $88-99$ & 90.5 & 5 & 0 & --- \\
\hline Ethenylbenzene & 77128 & 4 & $81-89$ & 83.5 & 3 & 0 & --- \\
\hline 1,2-Dimethylbenzene & 77135 & 4 & 89-94 & 90 & 3 & 0 & --- \\
\hline 1,1-Dichloropropene & 77168 & 4 & $85-90$ & 86 & 3 & 0 & --- \\
\hline 2,2-Dichloropropane & 77170 & 4 & $60-65$ & 64 & 1 & 0 & --- \\
\hline 1,3-Dichloropropane & 77173 & 4 & $88-95$ & 91.5 & 4 & 0 & --- \\
\hline 2-Ethyltoluene & 77220 & 4 & $85-92$ & 89 & 3 & 0 & --- \\
\hline 1,2,3-Trimethylbenzene & 77221 & 4 & $92-101$ & 97.5 & 3 & 0 & --- \\
\hline 1,2,4-Trimethylbenzene & 77222 & 4 & $90-93$ & 91.5 & 2 & 0 & --- \\
\hline (1-Methylethyl)benzene & 77223 & 4 & $85-91$ & 86.5 & 4 & 0 & --- \\
\hline n-Propylbenzene & 77224 & 4 & $83-88$ & 85 & 4 & 0 & --- \\
\hline 1,3,5-Trimethylbenzene & 77226 & 4 & $83-90$ & 88 & 5 & 0 & --- \\
\hline 1-Chloro-2-methylbenzene & 77275 & 4 & $84-88$ & 86 & 3 & 0 & --- \\
\hline 1-Chloro-4-methylbenzene & 77277 & 4 & $81-88$ & 83 & 3 & 0 & --- \\
\hline n-Butylbenzene & 77342 & 4 & $75-78$ & 76.5 & 2 & 0 & --- \\
\hline (1-Methylpropyl)benzene & 77350 & 4 & $84-88$ & 85 & 3 & 0 & --- \\
\hline (1,1-Dimethylethyl)benzene & 77353 & 4 & $85-90$ & 86.5 & 4 & 0 & --- \\
\hline 1-Isopropyl-4-methylbenzene & 77356 & 4 & $85-90$ & 87 & 3 & 0 & --- \\
\hline Iodomethane & 77424 & 4 & $81-102$ & 86.5 & 12 & 0 & --- \\
\hline 1,2,3-Trichloropropane & 77443 & 4 & $86-96$ & 91.5 & 3 & 0 & --- \\
\hline 1,1,1,2-Tetrachloroethane & 77562 & 4 & $81-89$ & 85 & 5 & 0 & --- \\
\hline 1,2,3-Trichlorobenzene & 77613 & 4 & $95-104$ & 100.5 & 5 & 0 & --- \\
\hline 1,2-Dibromomethane & 77651 & 4 & $81-91$ & 85.5 & 8 & 0 & --- \\
\hline
\end{tabular}


Table 24. Percent recovery statistics for volatile organic compounds detected in field-matrix spike samples for ground-water and surfacewater samples, Central Arizona Basins study area, 1996-98-Continued

\begin{tabular}{|c|c|c|c|c|c|c|c|}
\hline \multirow[b]{2}{*}{ Volatile organic compound } & \multirow[b]{2}{*}{$\begin{array}{l}\text { WATSTORE } \\
\text { code }\end{array}$} & \multicolumn{4}{|c|}{ Ground-water samples } & \multicolumn{2}{|c|}{ Surface-water sample } \\
\hline & & $\mathbf{n}$ & $\begin{array}{l}\text { Range of } \\
\text { recoveries, in } \\
\text { percent }\end{array}$ & $\begin{array}{l}\text { Median } \\
\text { recovery, in } \\
\text { percent }\end{array}$ & $\begin{array}{l}\text { Inter- } \\
\text { quartile } \\
\text { range }\end{array}$ & $\mathbf{n}$ & $\begin{array}{l}\text { Recovery, in } \\
\text { percent }\end{array}$ \\
\hline $\begin{array}{l}\text { 1,1,2-Trichloro-1,2,2- } \\
\text { trifluoroethane }\end{array}$ & 77652 & 4 & $66-70$ & 70 & 1 & 0 & --- \\
\hline Methyl tert-butyl ether & 78032 & 25 & $60-131$ & 87 & 24 & 1 & 69 \\
\hline 3-Chloro-1-propene & 78109 & 4 & $77-81$ & 80 & 1 & 0 & --- \\
\hline 4-Methyl-2-pentanone & 78133 & 4 & $80-90$ & 84.5 & 3 & 0 & --- \\
\hline Xylenes, Total & 81551 & --- & ---- & ---- & --- & 0 & --- \\
\hline Acetone & 81552 & 4 & $81-90$ & 86.5 & 6 & 0 & --- \\
\hline Bromobenzene & 81555 & 4 & $82-87$ & 84.5 & 2 & 0 & --- \\
\hline Diethyl ether & 81576 & 4 & $64-70$ & 68 & 3 & 0 & --- \\
\hline Diisopropyl ether & 81577 & 4 & $80-86$ & 83.5 & 4 & 0 & --- \\
\hline Methyl acrylonitrile & 81593 & 4 & $82-89$ & 85.5 & 6 & 0 & --- \\
\hline 2-Butanone & 81595 & 4 & $80-90$ & 84.5 & 6 & 0 & --- \\
\hline Methyl methacrylate & 81597 & 4 & $64-79$ & 70.5 & 5 & 0 & --- \\
\hline Tetrahydrofuran & 81607 & 4 & $67-80$ & 71 & 6 & 0 & --- \\
\hline 1,2-Dibromo-3-chloropropane & 82625 & 4 & $83-95$ & 85 & 5 & 0 & --- \\
\hline $\begin{array}{l}\text { 1,3-Dimethylbenzene } \\
\text { and 1,4-Dimethylbenzene }\end{array}$ & 85795 & 4 & $87-90$ & 88.5 & 2 & 0 & --- \\
\hline
\end{tabular}


Table 25. Pesticide and pesticide-degradation compounds detected in field blanks for surface-water samples, Central Arizona Basins study area, 1996-98

[Concentrations in micrograms per liter unless otherwise indicated. Bold type indicates results where the compound was present in sample. <, less than]

\begin{tabular}{|c|c|c|c|c|c|c|c|}
\hline Constituent & $\begin{array}{l}\text { Buckeye } \\
\text { Canal near } \\
\text { Avondale }\end{array}$ & $\begin{array}{c}\text { Hassayampa } \\
\text { River near } \\
\text { Arlington }\end{array}$ & $\begin{array}{l}\text { San Pedro } \\
\text { River at } \\
\text { Charleston }\end{array}$ & $\begin{array}{c}\text { Hassayampa } \\
\text { River near } \\
\text { Arlington }\end{array}$ & $\begin{array}{l}\text { Buckeye Canal } \\
\text { near Avondale }\end{array}$ & $\begin{array}{c}\text { Hassayampa } \\
\text { River near } \\
\text { Arlington }\end{array}$ & $\begin{array}{c}\text { Hassayampa } \\
\text { River near } \\
\text { Arlington }\end{array}$ \\
\hline Station site number & 09514000 & 09517000 & 09471000 & 09517000 & 09514000 & 09517000 & 09517000 \\
\hline Sample date & $12-17-96$ & $12-20-96$ & $03-25-97$ & 04-22-97 & $11-20-97$ & $12-02-97$ & $01-21-98$ \\
\hline Sample time & 0815 & 1350 & 1400 & 1130 & 0900 & 1030 & 0930 \\
\hline Acetochlor & $<0.002$ & $<0.002$ & $<0.002$ & $<0.002$ & $<0.002$ & $<0.002$ & $<0.002$ \\
\hline Acifluorfen & $<.035$ & $<.035$ & $<.035$ & $<.035$ & $<.035$ & $<.035$ & $<.035$ \\
\hline Alachlor & $<.002$ & $<.002$ & $<.002$ & $<.002$ & $<.002$ & $<.002$ & $<.002$ \\
\hline Aldicarb & $<.016$ & $<.016$ & $<.016$ & $<.016$ & $<.55$ & $<.55$ & $<.55$ \\
\hline Aldicarb sulfone & $<.016$ & $<.016$ & $<.016$ & $<.016$ & $<.1$ & $<.1$ & $<.1$ \\
\hline Aldicarb sulfoxide & $<.021$ & $<.021$ & $<.021$ & $<.021$ & $<.021$ & $<.021$ & $<.021$ \\
\hline Atrazine & $<.001$ & $<.001$ & $<.001$ & $<.001$ & $<.001$ & $<.001$ & $<.001$ \\
\hline Azinphos-methyl & $<.001$ & $<.001$ & $<.001$ & $<.001$ & $<.001$ & $<.001$ & $<.001$ \\
\hline Benfluralin & $<.002$ & $<.002$ & $<.002$ & $<.002$ & $<.002$ & $<.002$ & $<.002$ \\
\hline Bentazon & $<.014$ & $<.014$ & $<.014$ & $<.014$ & $<.014$ & $<.014$ & $<.014$ \\
\hline Bromacil & $<.035$ & $<.035$ & $<.035$ & $<.035$ & $<.035$ & $<.035$ & $<.035$ \\
\hline Bromoxynil & $<.035$ & $<.035$ & $<.035$ & $<.035$ & $<.035$ & $<.035$ & $<.035$ \\
\hline Butylate & $<.002$ & $<.002$ & $<.002$ & $<.002$ & $<.002$ & $<.002$ & $<.002$ \\
\hline Carbaryl $^{2}$ & $<.008$ & $<.008$ & $<.008$ & $<.008$ & $<.008$ & $<.008$ & $<.008$ \\
\hline Carbaryl $^{3}$ & $<.003$ & $<.003$ & $<.003$ & $<.003$ & $<.003$ & $<.003$ & $<.003$ \\
\hline Carbofuran $^{2}$ & $<.028$ & $<.028$ & $<.028$ & $<.028$ & $<.12$ & $<.12$ & $<.12$ \\
\hline Carbofuran $^{3}$ & $<.003$ & $<.003$ & $<.003$ & $<.003$ & $<.003$ & $<.003$ & $<.003$ \\
\hline Chloramben & $<.011$ & $<.011$ & $<.011$ & $<.011$ & $<.42$ & $<.42$ & $<.42$ \\
\hline Chlorothalonil & $<.035$ & $<.035$ & $<.035$ & $<.035$ & $<.48$ & $<.48$ & $<.48$ \\
\hline Chlorpyrifos & $<.004$ & $<.004$ & $<.004$ & $<.004$ & $<.004$ & $<.004$ & $<.004$ \\
\hline Clopyralid & $<.05$ & $<.05$ & $<.05$ & $<.05$ & $<.23$ & $<.23$ & $<.23$ \\
\hline Cyanazine & $<.004$ & $<.004$ & $<.004$ & $<.004$ & $<.004$ & $<.004$ & $<.004$ \\
\hline 2,4-D & $<.035$ & $<.035$ & $<.035$ & $<.035$ & $<.15$ & $<.15$ & $<.15$ \\
\hline Dacthal & $<.002$ & $<.002$ & $<.002$ & $<.002$ & $<.002$ & $<.002$ & $<.002$ \\
\hline Dacthal mono-acid & $<.017$ & $<.017$ & $<.017$ & $<.017$ & $<.017$ & $<.017$ & $<.017$ \\
\hline 2,4-DB & $<.035$ & $<.035$ & $<.035$ & $<.035$ & $<.24$ & $<.24$ & $<.24$ \\
\hline $\mathrm{p}, \mathrm{p}^{\prime}-\mathrm{DDE}$ & $<.006$ & $<.006$ & $<.006$ & ${ }^{1} .00184$ & $<.006$ & $<.006$ & $<.006$ \\
\hline Deethylatrazine & $<.002$ & $<.002$ & $<.002$ & $<.002$ & $<.002$ & $<.002$ & $<.002$ \\
\hline Diazinon & $<.002$ & $<.002$ & $<.002$ & $<.002$ & $<.002$ & $<.002$ & $<.002$ \\
\hline Dicamba & $<.035$ & $<.035$ & $<.035$ & $<.035$ & $<.035$ & $<.035$ & $<.035$ \\
\hline Dichlobenil & $<.02$ & $<.02$ & $<.02$ & $<.02$ & $<1.2$ & $<1.2$ & $<1.2$ \\
\hline Dichlorprop & $<.032$ & $<.032$ & $<.032$ & $<.032$ & $<.032$ & $<.032$ & $<.032$ \\
\hline Dieldrin & $<.001$ & $<.001$ & $<.001$ & $<.001$ & $<.001$ & $<.001$ & $<.001$ \\
\hline
\end{tabular}

See footnotes at end of table. 
Table 25. Pesticide and pesticide-degradation compounds detected in field blanks for surface-water samples, Central Arizona Basins study area, 1996-98-Continued

\begin{tabular}{|c|c|c|c|c|c|c|c|}
\hline Constituent & $\begin{array}{l}\text { Buckeye } \\
\text { Canal near } \\
\text { Avondale }\end{array}$ & $\begin{array}{c}\text { Hassayampa } \\
\text { River near } \\
\text { Arlington }\end{array}$ & $\begin{array}{c}\text { San Pedro } \\
\text { River at } \\
\text { Charleston }\end{array}$ & $\begin{array}{c}\text { Hassayampa } \\
\text { River near } \\
\text { Arlington }\end{array}$ & $\begin{array}{l}\text { Buckeye } \\
\text { Canal near } \\
\text { Avondale }\end{array}$ & $\begin{array}{c}\text { Hassayampa } \\
\text { River near } \\
\text { Arlington }\end{array}$ & $\begin{array}{c}\text { Hassayampa } \\
\text { River near } \\
\text { Arlington }\end{array}$ \\
\hline 2,6-Diethylaniline & $<0.003$ & $<0.003$ & $<0.003$ & $<0.003$ & $<0.003$ & $<0.003$ & $<0.003$ \\
\hline Dinoseb & $<.035$ & $<.035$ & $<.035$ & $<.035$ & $<.035$ & $<.035$ & $<.035$ \\
\hline Disulfoton & $<.017$ & $<.017$ & $<.017$ & $<.017$ & $<.017$ & $<.017$ & $<.017$ \\
\hline Diuron & $<.02$ & $<.02$ & $<.02$ & $<.02$ & $<.02$ & $<.02$ & $<.02$ \\
\hline DNOC & $<.035$ & $<.035$ & $<.035$ & $<.035$ & $<.42$ & $<.42$ & $<.42$ \\
\hline EPTC & $<.002$ & $<.002$ & $<.002$ & $<.002$ & $<.002$ & $<.002$ & $<.002$ \\
\hline Ethalfluralin & $<.004$ & $<.004$ & $<.004$ & $<.004$ & $<.004$ & $<.004$ & $<.004$ \\
\hline Ethoprop & $<.003$ & $<.003$ & $<.003$ & $<.003$ & $<.003$ & $<.003$ & $<.003$ \\
\hline Fenuron & $<.013$ & $<.013$ & $<.013$ & $<.013$ & $<.013$ & $<.013$ & $<.013$ \\
\hline Fluometuron & $<.035$ & $<.035$ & $<.035$ & $<.035$ & $<.035$ & $<.035$ & $<.035$ \\
\hline Fonofos & $<.003$ & $<.003$ & $<.003$ & $<.003$ & $<.003$ & $<.003$ & $<.003$ \\
\hline alpha-HCH & $<.002$ & $<.002$ & $<.002$ & $<.002$ & $<.002$ & $<.002$ & $<.002$ \\
\hline gamma-HCH & $<.004$ & $<.004$ & $<.004$ & $<.004$ & $<.004$ & $<.004$ & $<.004$ \\
\hline 3-Hydroxycarbofuran & $<.014$ & $<.014$ & $<.014$ & $<.014$ & $<.014$ & $<.014$ & $<.014$ \\
\hline Linuron $^{2}$ & $<.018$ & $<.018$ & $<.018$ & $<.018$ & $<.018$ & $<.018$ & $<.018$ \\
\hline Linuron $^{3}$ & $<.002$ & $<.002$ & $<.002$ & $<.002$ & $<.002$ & $<.002$ & $<.002$ \\
\hline MCPA & $<.05$ & $<.05$ & $<.05$ & $<.05$ & $<.17$ & $<.17$ & $<.17$ \\
\hline МCPB & $<.035$ & $<.035$ & $<.035$ & $<.035$ & $<.14$ & $<.14$ & $<.14$ \\
\hline Malathion & $<.005$ & $<.005$ & $<.005$ & $<.005$ & $<.005$ & $<.005$ & $<.005$ \\
\hline Methiocarb & $<.026$ & $<.026$ & $<.026$ & $<.026$ & $<.026$ & $<.026$ & $<.026$ \\
\hline Methomyl & $<.017$ & $<.017$ & $<.017$ & $<.017$ & $<.017$ & $<.017$ & $<.017$ \\
\hline Methyl parathion & $<.006$ & $<.006$ & $<.006$ & $<.006$ & $<.006$ & $<.006$ & $<.006$ \\
\hline Metolachlor & $<.002$ & $<.002$ & $<.002$ & $<.002$ & $<.002$ & $<.002$ & $<.002$ \\
\hline Metribuzin & $<.004$ & $<.004$ & $<.004$ & $<.004$ & $<.004$ & $<.004$ & $<.004$ \\
\hline Molinate & $<.004$ & $<.004$ & $<.004$ & $<.004$ & $<.004$ & $<.004$ & $<.004$ \\
\hline Napropamide & $<.003$ & $<.003$ & $<.003$ & $<.003$ & $<.003$ & $<.003$ & $<.003$ \\
\hline Neburon & $<.015$ & $<.015$ & $<.015$ & $<.015$ & $<.015$ & $<.015$ & $<.015$ \\
\hline Norflurazon & $<.024$ & $<.024$ & $<.024$ & $<.024$ & $<.024$ & $<.024$ & $<.024$ \\
\hline Oryzalin & $<.019$ & $<.019$ & $<.019$ & $<.019$ & $<.31$ & $<.31$ & $<.31$ \\
\hline Oxamyl & $<.018$ & $<.018$ & $<.018$ & $<.018$ & $<.018$ & $<.018$ & $<.018$ \\
\hline Parathion & $<.004$ & $<.004$ & $<.004$ & $<.004$ & $<.004$ & $<.004$ & $<.004$ \\
\hline Pebulate & $<.004$ & $<.004$ & $<.004$ & $<.004$ & $<.004$ & $<.004$ & $<.004$ \\
\hline Pendimethalin & $<.004$ & $<.004$ & $<.004$ & $<.004$ & $<.004$ & $<.004$ & $<.004$ \\
\hline cis-Permethrin & $<.005$ & $<.005$ & $<.005$ & $<.005$ & $<.005$ & $<.005$ & $<.005$ \\
\hline Phorate & $<.002$ & $<.002$ & $<.002$ & $<.002$ & $<.002$ & $<.002$ & $<.002$ \\
\hline Picloram & $<.05$ & $<.05$ & $<.05$ & $<.05$ & $<.05$ & $<.05$ & $<.05$ \\
\hline Prometon & $<.018$ & $<.018$ & $<.018$ & $<.018$ & $<.018$ & $<.018$ & $<.018$ \\
\hline Pronamide & $<.003$ & $<.003$ & $<.003$ & $<.003$ & $<.003$ & $<.003$ & $<.003$ \\
\hline
\end{tabular}


Table 25. Pesticide and pesticide-degradation compounds detected in field blanks for surface-water samples, Central Arizona Basins study area, 1996-98-Continued

\begin{tabular}{|c|c|c|c|c|c|c|c|}
\hline Constituent & $\begin{array}{l}\text { Buckeye } \\
\text { Canal near } \\
\text { Avondale }\end{array}$ & $\begin{array}{c}\text { Hassayampa } \\
\text { River near } \\
\text { Arlington }\end{array}$ & $\begin{array}{l}\text { San Pedro } \\
\text { River at } \\
\text { Charleston }\end{array}$ & $\begin{array}{c}\text { Hassayampa } \\
\text { River near } \\
\text { Arlington }\end{array}$ & $\begin{array}{l}\text { Buckeye } \\
\text { Canal near } \\
\text { Avondale }\end{array}$ & $\begin{array}{c}\text { Hassayampa } \\
\text { River near } \\
\text { Arlington }\end{array}$ & $\begin{array}{c}\text { Hassayampa } \\
\text { River near } \\
\text { Arlington }\end{array}$ \\
\hline \multicolumn{8}{|c|}{ See footnotes at end of table. } \\
\hline Propachlor & $<0.007$ & $<0.007$ & $<0.007$ & $<0.007$ & $<0.007$ & $<0.007$ & $<0.007$ \\
\hline Propanil & $<.004$ & $<.004$ & $<.004$ & $<.004$ & $<.004$ & $<.004$ & $<.004$ \\
\hline Propargite & $<.013$ & $<.013$ & $<.013$ & $<.013$ & $<.013$ & $<.013$ & $<.013$ \\
\hline Propham & $<.035$ & $<.035$ & $<.035$ & $<.035$ & $<.035$ & $<.035$ & $<.035$ \\
\hline Propoxur & $<.035$ & $<.035$ & $<.035$ & $<.035$ & $<.035$ & $<.035$ & $<.035$ \\
\hline Silvex & $<.021$ & $<.021$ & $<.021$ & $<.021$ & $<.021$ & $<.021$ & $<.021$ \\
\hline Simazine & $<.005$ & $<.005$ & $<.005$ & $<.005$ & $<.005$ & $<.005$ & $<.005$ \\
\hline $2,4,5-\mathrm{T}$ & $<.035$ & $<.035$ & $<.035$ & $<.035$ & $<.035$ & $<.035$ & $<.035$ \\
\hline Tebuthiuron & $<.01$ & $<.01$ & $<.01$ & $<.01$ & $<.01$ & $<.01$ & $<.01$ \\
\hline Terbacil & $<.007$ & $<.007$ & $<.007$ & $<.007$ & $<.007$ & $<.007$ & $<.007$ \\
\hline Terbufos & $<.013$ & $<.013$ & $<.013$ & $<.013$ & $<.013$ & $<.013$ & $<.013$ \\
\hline Thiobencarb & $<.002$ & $<.002$ & $<.002$ & $<.002$ & $<.002$ & $<.002$ & $<.002$ \\
\hline Triallate & $<.001$ & $<.001$ & $<.001$ & $<.001$ & $<.001$ & $<.001$ & $<.001$ \\
\hline Triclopyr & $<.05$ & $<.05$ & $<.05$ & $<.05$ & $<.25$ & $<.25$ & $<.25$ \\
\hline Trifluralin & $<.002$ & $<.002$ & $<.002$ & $<.002$ & $<.002$ & $<.002$ & $<.002$ \\
\hline \multicolumn{8}{|c|}{ Surrogate recoveries, in percent } \\
\hline BDMC & 69 & 36 & 94 & 76 & 73 & 69 & 86 \\
\hline Diazinon-D10 & 86 & 87 & 99 & 82 & 103 & 94 & 95 \\
\hline Terbuthylazine & 87 & 100 & 109 & 89 & 116 & 107 & 106 \\
\hline Alpha-HCH-D6 & 78 & 75 & 106 & 71 & 86 & 78 & 84 \\
\hline
\end{tabular}

${ }^{1}$ Estimated.

${ }^{2}$ Compound analyzed by gas chromatography/mass spectrometry.

${ }^{3}$ Compound analyzed by high-performance liquid chromotography. 


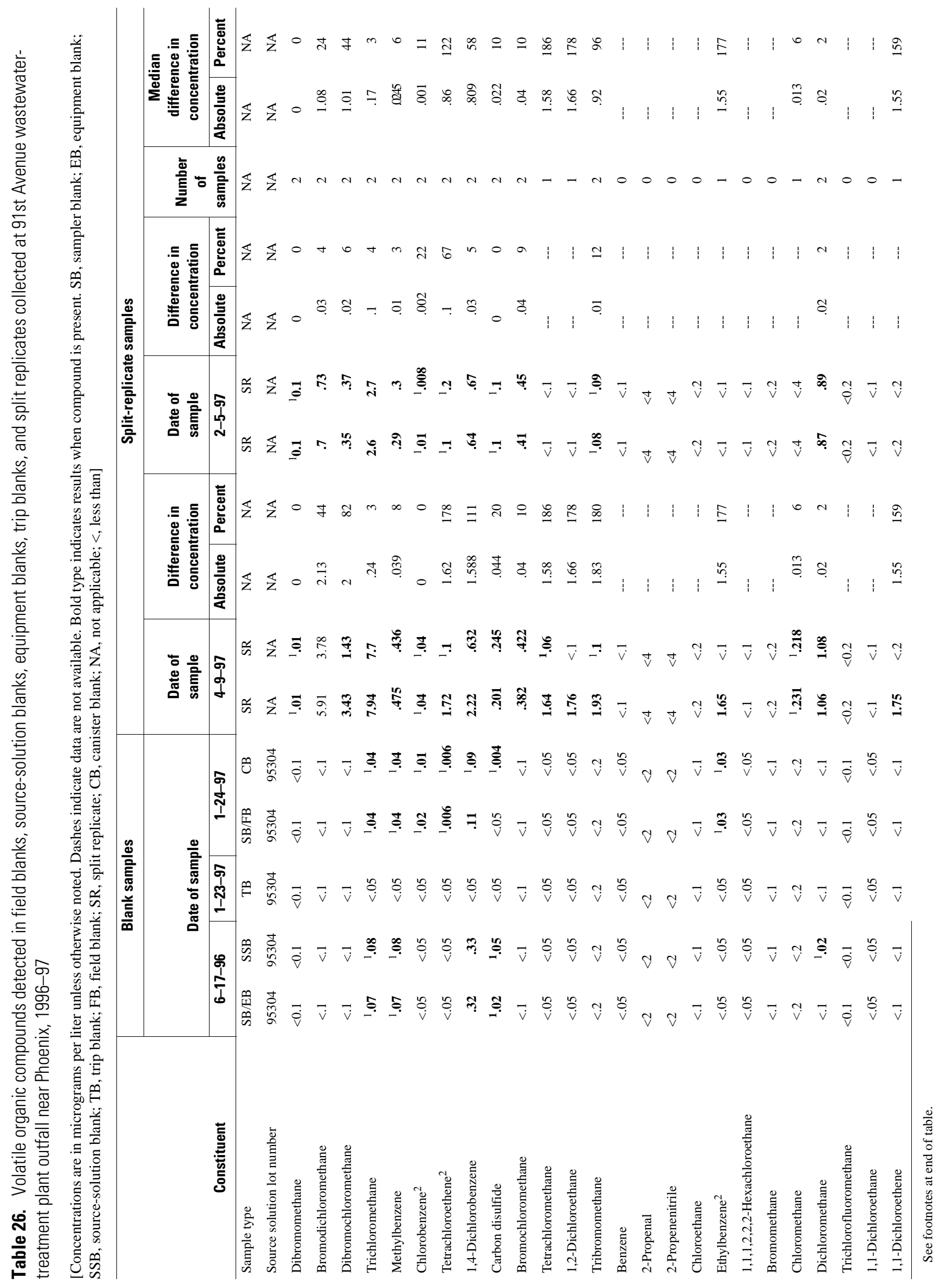




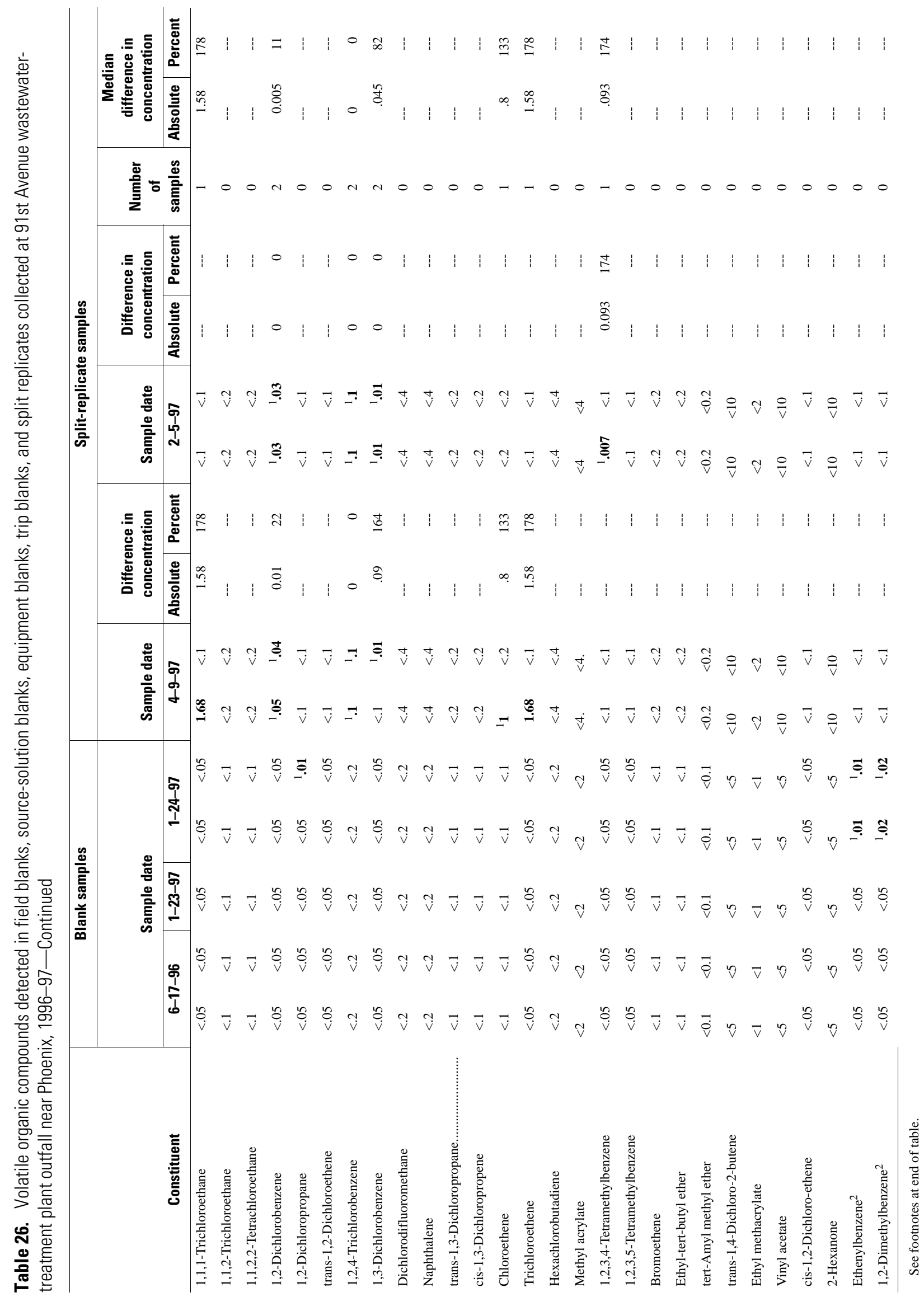




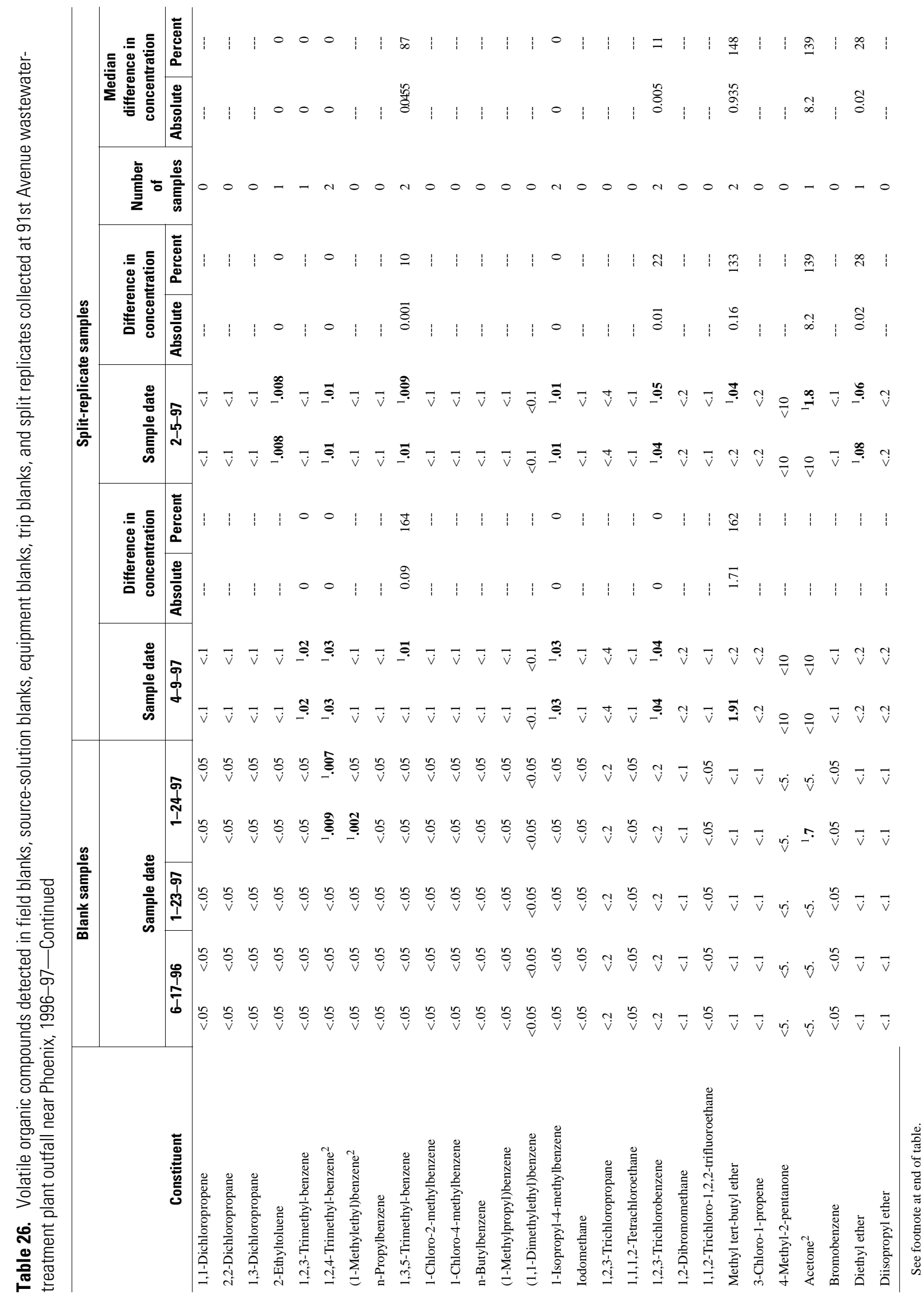




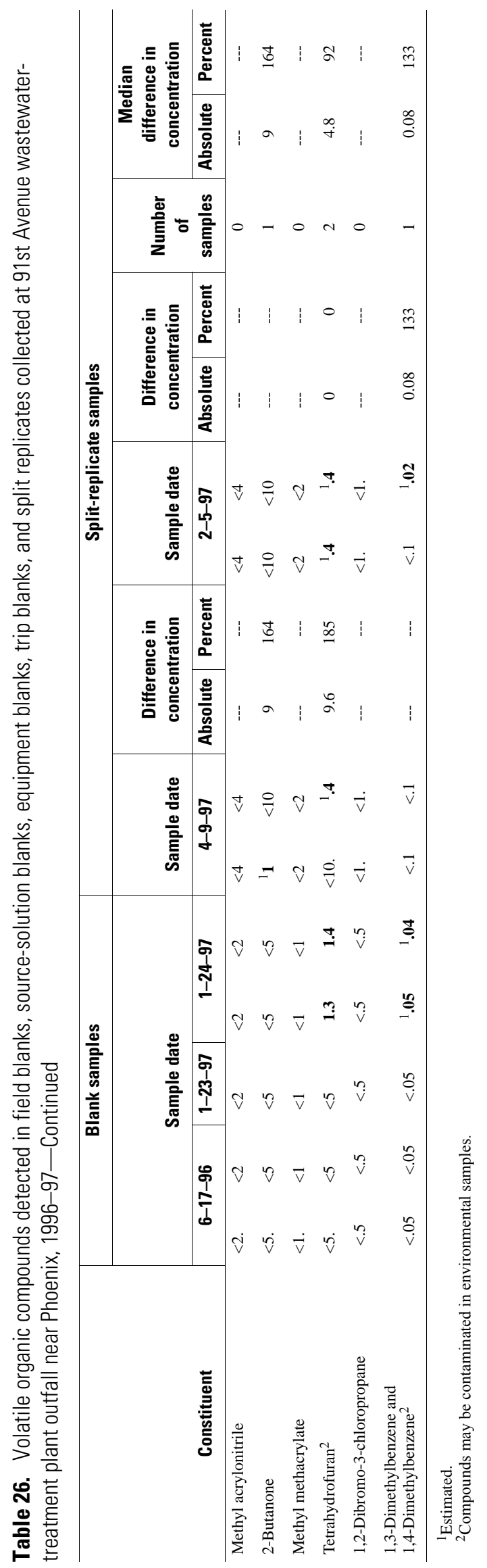

84 Characteristics of Shallow Deposits Beneath Rillito Creek, Pima County, Arizona 
Table 27. Summary statistics for pesticides and pesticide-degradation compounds detected in field-matrix spike samples and replicates for surface-water samples, Central Arizona Basins study area, 1996-98

[Concentrations are in micrograms per liter unless otherwise indicated. Methods of analysis for pesticide and pesticide-degradation compounds are listed in table 5. Compounds in bold font may be affected by matrix effects. Dashes indicate no data; n, number of field-matrix spike samples or number of replicatesample pairs; FMS-SS, field-matrix spike sample median minus the set-spike sample median (from table 22)]

\begin{tabular}{|c|c|c|c|c|c|c|c|c|c|c|c|c|c|c|}
\hline \multirow[b]{4}{*}{ Pesticide } & \multicolumn{4}{|c|}{ Field-matrix spike samples } & \multicolumn{10}{|c|}{ Replicate samples } \\
\hline & \multirow{2}{*}{\multicolumn{4}{|c|}{ Recoveries }} & \multicolumn{5}{|c|}{ Sequential replicates } & \multicolumn{5}{|c|}{ Concurrent replicates } \\
\hline & & & & & \multicolumn{5}{|c|}{ Difference between replicates } & \multicolumn{5}{|c|}{ Diference between replicates } \\
\hline & $=$ & 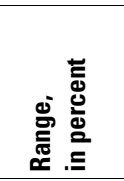 & 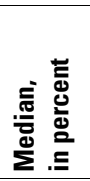 & 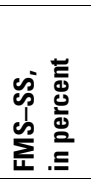 & $=$ & 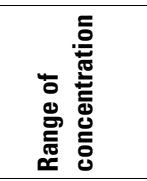 & 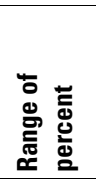 & 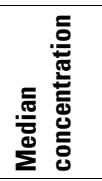 & 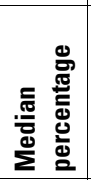 & $=$ & 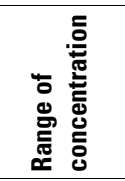 & 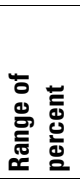 & 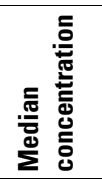 & 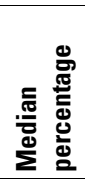 \\
\hline Acetochlor & 2 & $98-110$ & 104 & 2 & 0 & --- & --- & --- & --- & 0 & --- & --- & --- & --- \\
\hline Acifluorfen & 2 & $30-48$ & 39 & 49 & 0 & --- & --- & --- & --- & 0 & --- & --- & --- & --- \\
\hline Alachlor & 2 & $102-109$ & 106 & 4 & 0 & --- & --- & --- & --- & 0 & --- & --- & --- & --- \\
\hline Aldicarb $^{1}$ & 0 & --- & --- & --- & 0 & --- & --- & --- & --- & 0 & --- & --- & --- & --- \\
\hline Aldicarb sulfone ${ }^{1}$ & 0 & --- & --- & --- & 0 & --- & --- & --- & --- & 0 & --- & --- & --- & --- \\
\hline Aldicarb sulfoxide ${ }^{1}$ & 0 & --- & --- & --- & 0 & --- & --- & --- & --- & 0 & --- & --- & --- & --- \\
\hline Atrazine & 2 & $93-106$ & 100 & 0 & 4 & $\begin{array}{c}0.0004- \\
.00466\end{array}$ & $2-140$ & 0.0018 & 10 & 1 & 0.0013 & 7 & --- & --- \\
\hline Azinphos-methyl & 1 & 221 & --- & 141 & 0 & --- & --- & --- & --- & 0 & --- & --- & --- & --- \\
\hline Benfluralin & 2 & $81-150$ & 116 & 49 & 1 & .0026 & 12 & --- & --- & 0 & --- & --- & --- & --- \\
\hline Bentazon & 2 & $43-47$ & 45 & 35 & 0 & --- & --- & --- & --- & 0 & --- & --- & --- & --- \\
\hline Bromacil & 1 & 130 & --- & 48 & 0 & --- & --- & --- & --- & 0 & --- & --- & --- & --- \\
\hline Bromoxynil & 2 & $83-84$ & 84 & 1 & 0 & --- & --- & --- & --- & 0 & --- & --- & --- & --- \\
\hline Butylate & 2 & $113-124$ & 118 & 29 & 0 & --- & --- & --- & --- & 0 & --- & --- & --- & --- \\
\hline Carbaryl $^{1,2}$ & 2 & $178-318$ & 248 & 122 & 0 & --- & --- & --- & --- & 0 & --- & --- & --- & --- \\
\hline Carbaryl $^{3}$ & 1 & 50 & --- & 38 & 3 & $.002-.007$ & $6-31$ & .0034 & 9 & 1 & .0005 & 2 & --- & --- \\
\hline Carbofuran $^{2}$ & 2 & $165-183$ & 174 & 61 & 0 & --- & --- & --- & --- & 0 & --- & --- & --- & --- \\
\hline Carbofuran $^{3}$ & 1 & 104 & --- & 21 & 0 & --- & --- & --- & --- & 0 & --- & --- & --- & --- \\
\hline Chloramben & 1 & 0 & --- & 70 & 0 & --- & --- & --- & --- & 0 & --- & --- & --- & --- \\
\hline Chlorothalonil $^{1}$ & 1 & 27 & --- & 9 & 0 & --- & --- & --- & --- & 0 & --- & --- & --- & --- \\
\hline Chlorpyrifos & 2 & 97 & 97 & 7 & 1 & $0-.025$ & $0-62$ & .00322 & 7 & 4 & $0-.00815$ & $0-20$ & 0.0024 & 11 \\
\hline Clopyralid & 1 & 0 & --- & 77 & 0 & --- & --- & --- & --- & 0 & --- & --- & --- & --- \\
\hline Cyanazine & 2 & $80-126$ & 103 & 0 & 4 & $.0006-.018$ & $1-138$ & .00437 & 20 & 1 & .00185 & 3 & --- & --- \\
\hline 2,4-D & 0 & --- & --- & --- & 0 & --- & --- & --- & --- & 0 & --- & --- & --- & --- \\
\hline Dacthal & 2 & $102-112$ & 107 & 4 & 9 & $0-.0031$ & $0-13$ & .00007 & 6 & 3 & $0-.005$ & $0-10$ & .00012 & 7 \\
\hline Dacthal mono-acid & 2 & $51-64$ & 58 & 25 & 0 & --- & --- & --- & --- & 0 & --- & --- & --- & \\
\hline 2,4-DB & 0 & --- & --- & --- & 0 & --- & --- & --- & --- & 0 & --- & --- & --- & \\
\hline p,p'-DDE & 2 & $46-47$ & 46 & 12 & 9 & $0-.00231$ & $0-73$ & .00102 & 22 & 2 & $0-.0005$ & $0-12$ & .00025 & 6 \\
\hline Deethylatrazine $^{1}$ & 2 & $34-37$ & 36 & 9 & 3 & $\begin{array}{l}.0009- \\
.00199\end{array}$ & $10-25$ & .00099 & 16 & 1 & .001515 & 17 & --- & \\
\hline Diazinon & 2 & $91-102$ & 96 & 8 & 1 & $.0005-.011$ & $2-36$ & .005 & 7 & 4 & $0-.00425$ & $0-21$ & .00175 & 2 \\
\hline Dicamba & 0 & --- & --- & --- & 0 & --- & --- & --- & --- & 0 & --- & --- & --- & --- \\
\hline Dichlobenil $^{1}$ & 0 & --- & --- & --- & 0 & --- & --- & --- & --- & 0 & --- & --- & --- & --- \\
\hline Dichlorprop & 2 & $69-136$ & 102 & 17 & 0 & --- & --- & --- & --- & 0 & --- & --- & --- & --- \\
\hline
\end{tabular}


Table 27. Summary statistics for pesticides and pesticide-degradation compounds detected in field-matrix spike samples and replicates for surface-water samples, Central Arizona Basins study area, 1996-98-Continued

\begin{tabular}{|c|c|c|c|c|c|c|c|c|c|c|c|c|c|c|}
\hline \multirow[b]{4}{*}{ Pesticide } & \multicolumn{4}{|c|}{ Field-matrix spike samples } & \multicolumn{10}{|c|}{ Replicate samples } \\
\hline & \multirow{2}{*}{\multicolumn{4}{|c|}{ Recoveries }} & \multicolumn{5}{|c|}{ Sequential replicates } & \multicolumn{5}{|c|}{ Concurrent replicates } \\
\hline & & & & & \multicolumn{5}{|c|}{$\begin{array}{c}\text { Difference, in } \\
\text { concentration, in percent }\end{array}$} & \multicolumn{5}{|c|}{$\begin{array}{c}\text { Difference, in } \\
\text { concentration, in percent }\end{array}$} \\
\hline & $=$ & 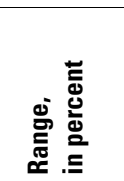 & 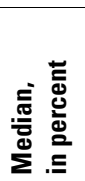 & 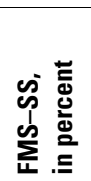 & $=$ & 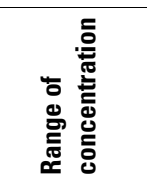 & 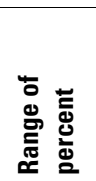 & 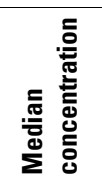 & 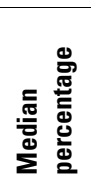 & $=$ & 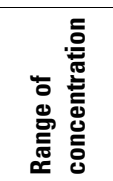 & 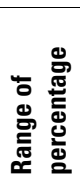 & 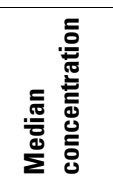 & 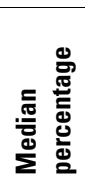 \\
\hline Dieldrin & 2 & $97-99$ & 98 & 17 & 0 & --- & --- & --- & --- & 0 & --- & --- & --- & --- \\
\hline 2,6-Diethyl-aniline & 2 & $81-88$ & 84 & 1 & 0 & --- & --- & --- & --- & 0 & --- & --- & --- & --- \\
\hline Dinoseb & 2 & $34-38$ & 36 & 45 & 0 & --- & --- & --- & --- & 0 & --- & --- & --- & --- \\
\hline Disulfoton & 2 & $38-90$ & 64 & 9 & 2 & $0.041-.053$ & $6-7$ & 0.047 & 6 & 1 & 0.057 & 7 & --- & --- \\
\hline Diuron & 0 & --- & --- & --- & 1 & .21 & 3 & --- & --- & 0 & --- & --- & --- & --- \\
\hline $\mathrm{DNOC}^{1}$ & 2 & $52-56$ & 54 & 15 & 0 & --- & --- & --- & --- & 0 & --- & --- & --- & --- \\
\hline EPTC & 2 & $0-146$ & 73 & 17 & 9 & $0-.088$ & $0-32$ & .0088 & 10 & 4 & $\begin{array}{l}.0005- \\
.0405\end{array}$ & $3-14$ & 0.00385 & 6 \\
\hline Ethalfluralin & 2 & $93-182$ & 138 & 60 & 0 & --- & --- & --- & --- & 0 & --- & --- & --- & --- \\
\hline Ethoprop & 2 & $104-124$ & 114 & 19 & 0 & --- & --- & --- & --- & 0 & --- & --- & --- & --- \\
\hline Fenuron & 1 & 50 & --- & 39 & 0 & --- & --- & --- & --- & 0 & --- & --- & --- & --- \\
\hline Fluometuron & 1 & 55 & --- & 38 & 0 & --- & --- & --- & --- & 0 & --- & --- & --- & --- \\
\hline Fonofos & 2 & $84-107$ & 96 & 8 & 0 & --- & --- & --- & --- & 0 & --- & --- & --- & --- \\
\hline alpha-HCH & 2 & $90-94$ & 92 & 2 & 0 & --- & --- & --- & --- & 0 & --- & --- & --- & --- \\
\hline gamma-HCH & 2 & $120-130$ & 125 & 33 & 3 & $\begin{array}{l}.0017- \\
.0028\end{array}$ & $13-24$ & .0025 & 17 & 1 & .00155 & 13 & --- & --- \\
\hline $\begin{array}{l}\text { 3- } \\
\text { Hydroxycarbofur } \\
\text { an }\end{array}$ & 1 & 49 & --- & 27 & 0 & --- & --- & --- & --- & 0 & --- & --- & --- & --- \\
\hline Linuron $^{2}$ & 2 & 116 & 116 & 15 & 2 & $.003-.005$ & $7-12$ & .004 & 10 & 0 & --- & --- & --- & --- \\
\hline Linuron $^{3}$ & 1 & 54 & --- & 31 & 0 & --- & --- & --- & --- & 1 & .001 & 2 & --- & --- \\
\hline MCPA & 1 & 43 & --- & 41 & 0 & --- & --- & --- & --- & 0 & --- & --- & --- & --- \\
\hline МСРВ & 0 & --- & --- & --- & 0 & --- & --- & --- & --- & 0 & --- & --- & --- & --- \\
\hline Malathion & 2 & $85-112$ & 98 & 3 & 4 & $.001-.011$ & $0-9$ & .005 & 4 & 2 & $\begin{array}{l}.0025- \\
.0085\end{array}$ & $2-3$ & .0055 & 3 \\
\hline Methiocarb & 1 & 49 & --- & 36 & 0 & --- & --- & --- & --- & 0 & --- & --- & --- & --- \\
\hline Methomyl & 1 & 51 & --- & 39 & 0 & --- & --- & --- & --- & 0 & --- & --- & --- & --- \\
\hline Methyl parathion & 2 & $105-171$ & 138 & 49 & 1 & .0101 & 33 & --- & --- & 0 & --- & --- & --- & --- \\
\hline Metolachlor & 2 & $116-146$ & 131 & 25 & 0 & --- & --- & --- & --- & 0 & --- & --- & --- & --- \\
\hline Metribuzin & 2 & 89-109 & 99 & 21 & 0 & --- & --- & --- & --- & 0 & --- & --- & --- & --- \\
\hline Molinate & 2 & $101-115$ & 108 & 14 & 0 & --- & --- & --- & --- & 0 & --- & --- & --- & --- \\
\hline Napropamide & 2 & $91-100$ & 96 & 2 & 0 & --- & --- & --- & --- & 0 & --- & --- & --- & --- \\
\hline Neburon & 1 & 32 & --- & 53 & 0 & --- & --- & --- & --- & 0 & --- & --- & --- & --- \\
\hline Norflurazon & 1 & 52 & --- & 36 & 0 & --- & --- & --- & --- & 0 & --- & --- & --- & --- \\
\hline Oryzalin & 0 & --- & --- & --- & 0 & --- & --- & --- & --- & 0 & --- & --- & --- & --- \\
\hline Oxamyl & 1 & 30 & --- & 47 & 0 & --- & --- & --- & --- & 0 & --- & --- & --- & --- \\
\hline Parathion & 2 & $100-173$ & 136 & 43 & 0 & --- & --- & --- & --- & 0 & --- & --- & --- & --- \\
\hline Pebulate & 2 & $107-115$ & 111 & 21 & 0 & --- & --- & --- & --- & 0 & --- & --- & --- & --- \\
\hline Pendimethalin & 2 & $7-155$ & 81 & 8 & 6 & $.0003-.009$ & $1-22$ & .0034 & 9 & 2 & $\begin{array}{l}.004- \\
.01145\end{array}$ & $5-32$ & .007725 & 18 \\
\hline cis-Permethrin & 2 & $25-31$ & 28 & 17 & 0 & --- & --- & --- & --- & 0 & --- & --- & --- & --- \\
\hline Phorate & 2 & $40-97$ & 68 & 4 & 2 & 0-.001 & $0-10$ & 0.0005 & 5 & 1 & 0.0005 & 5 & --- & \\
\hline Picloram & 2 & $47-57$ & 52 & 29 & 0 & --- & --- & --- & --- & 0 & --- & --- & --- & --- \\
\hline
\end{tabular}


Table 27. Summary statistics for pesticides and pesticide-degradation compounds detected in field-matrix spike samples and replicates for surface-water samples, Central Arizona Basins study area, 1996-98-Continued

\begin{tabular}{|c|c|c|c|c|c|c|c|c|c|c|c|c|c|c|}
\hline \multirow[b]{4}{*}{ Pesticide } & \multicolumn{4}{|c|}{ Field-matrix spike samples } & \multicolumn{10}{|c|}{ Replicate samples } \\
\hline & \multirow{2}{*}{\multicolumn{4}{|c|}{ Recoveries }} & \multicolumn{5}{|c|}{ Sequential replicates } & \multicolumn{5}{|c|}{ Concurrent replicates } \\
\hline & & & & & \multicolumn{4}{|c|}{$\begin{array}{c}\text { Difference, in } \\
\text { concentration, in percent }\end{array}$} & \multirow[b]{2}{*}{ 兽 } & \multicolumn{4}{|c|}{$\begin{array}{l}\text { Difference, in } \\
\text { concentration, in percent }\end{array}$} & \multirow[b]{2}{*}{ 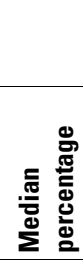 } \\
\hline & $=$ & 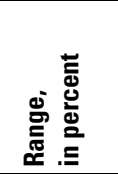 & 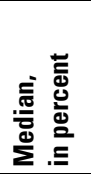 & 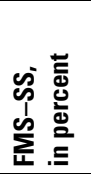 & $=$ & 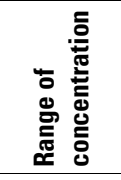 & 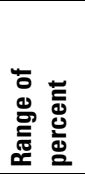 & 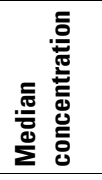 & & $=$ & 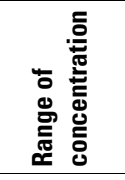 & 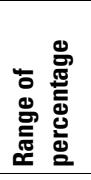 & 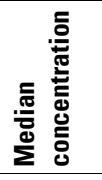 & \\
\hline \multicolumn{15}{|c|}{ See footnotes at end of table. } \\
\hline Prometon & 2 & $109-112$ & 110 & 39 & 9 & $0-.009$ & $0-67$ & .00156 & 12 & 3 & $\begin{array}{l}.0005- \\
.00398\end{array}$ & $2-35$ & .0025 & 9 \\
\hline Pronamide & 2 & $92-108$ & 100 & 11 & 0 & --- & --- & --- & --- & 0 & --- & --- & --- & --- \\
\hline Propachlor & 2 & $117-129$ & 123 & 18 & 0 & --- & --- & --- & --- & 0 & --- & --- & --- & --- \\
\hline Propanil & 2 & $106-125$ & 116 & 8 & 0 & --- & --- & --- & --- & 0 & --- & --- & --- & --- \\
\hline Propargite & 2 & $90-130$ & 110 & 39 & 0 & --- & --- & --- & --- & 0 & --- & --- & --- & --- \\
\hline Propham & 1 & 42 & ---- & 26 & 0 & --- & --- & --- & --- & 0 & --- & --- & --- & --- \\
\hline Propoxur & 1 & 81 & ---- & 8 & 0 & --- & --- & --- & --- & 0 & --- & --- & --- & --- \\
\hline Silvex & 1 & 80 & ---- & 8 & 0 & --- & --- & --- & --- & 0 & --- & --- & --- & --- \\
\hline Simazine & 2 & 101-106 & 104 & 3 & 8 & $\begin{array}{l}.0003- \\
.0073\end{array}$ & $2-84$ & .00112 & 11 & 2 & $\begin{array}{l}.0015- \\
.003\end{array}$ & $5-19$ & .00225 & 12 \\
\hline $2,4,5-\mathrm{T}$ & 1 & 59 & ---- & 23 & 0 & --- & --- & --- & --- & 0 & --- & --- & --- & --- \\
\hline Tebuthiuron & 2 & 109-179 & 144 & 28 & 0 & --- & --- & --- & --- & 0 & --- & --- & --- & --- \\
\hline Terbacil $^{1}$ & 2 & $75-78$ & 76 & 4 & 0 & --- & --- & --- & --- & 0 & --- & --- & --- & --- \\
\hline Terbufos & 2 & $80-103$ & 92 & 16 & 0 & --- & --- & --- & --- & 0 & --- & --- & --- & --- \\
\hline Thiobencarb & 2 & 85-102 & 94 & 6 & 0 & --- & --- & --- & --- & 0 & --- & --- & --- & --- \\
\hline Triallate & 2 & 80-97 & 88 & 1 & 0 & --- & --- & --- & --- & 0 & --- & --- & --- & --- \\
\hline Triclopyr & 0 & ---- & ---- & --- & 0 & --- & --- & --- & --- & 0 & --- & --- & --- & --- \\
\hline Trifluralin & 2 & $80-154$ & 117 & 46 & 1 & $\begin{array}{c}.00003- \\
.007\end{array}$ & $1-85$ & .00088 & 6 & 4 & $\begin{array}{c}.00024- \\
.008\end{array}$ & $2-40$ & .001623 & 9 \\
\hline
\end{tabular}

${ }^{1}$ Analytical performance for this compound is considered poor (J.D. Martin, hydrologist, U.S. Geological Survey, written commun., 1999).

${ }^{2}$ Compound analyzed by gas chromatography/mass spectrometry.

${ }^{3}$ Compound analyzed by high-performance liquid chromatography. 


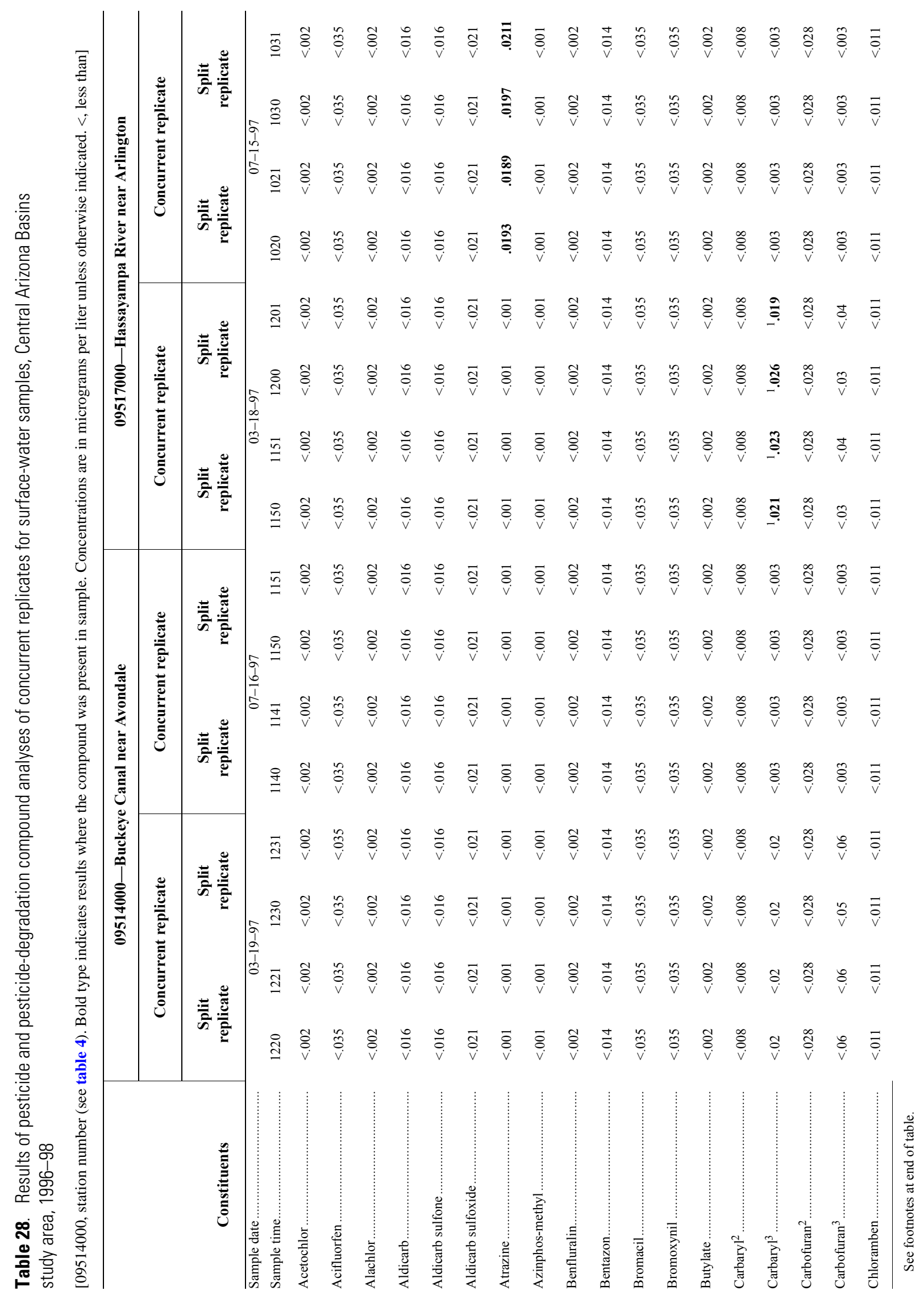

88 Characteristics of Shallow Deposits Beneath Rillito Creek, Pima County, Arizona 


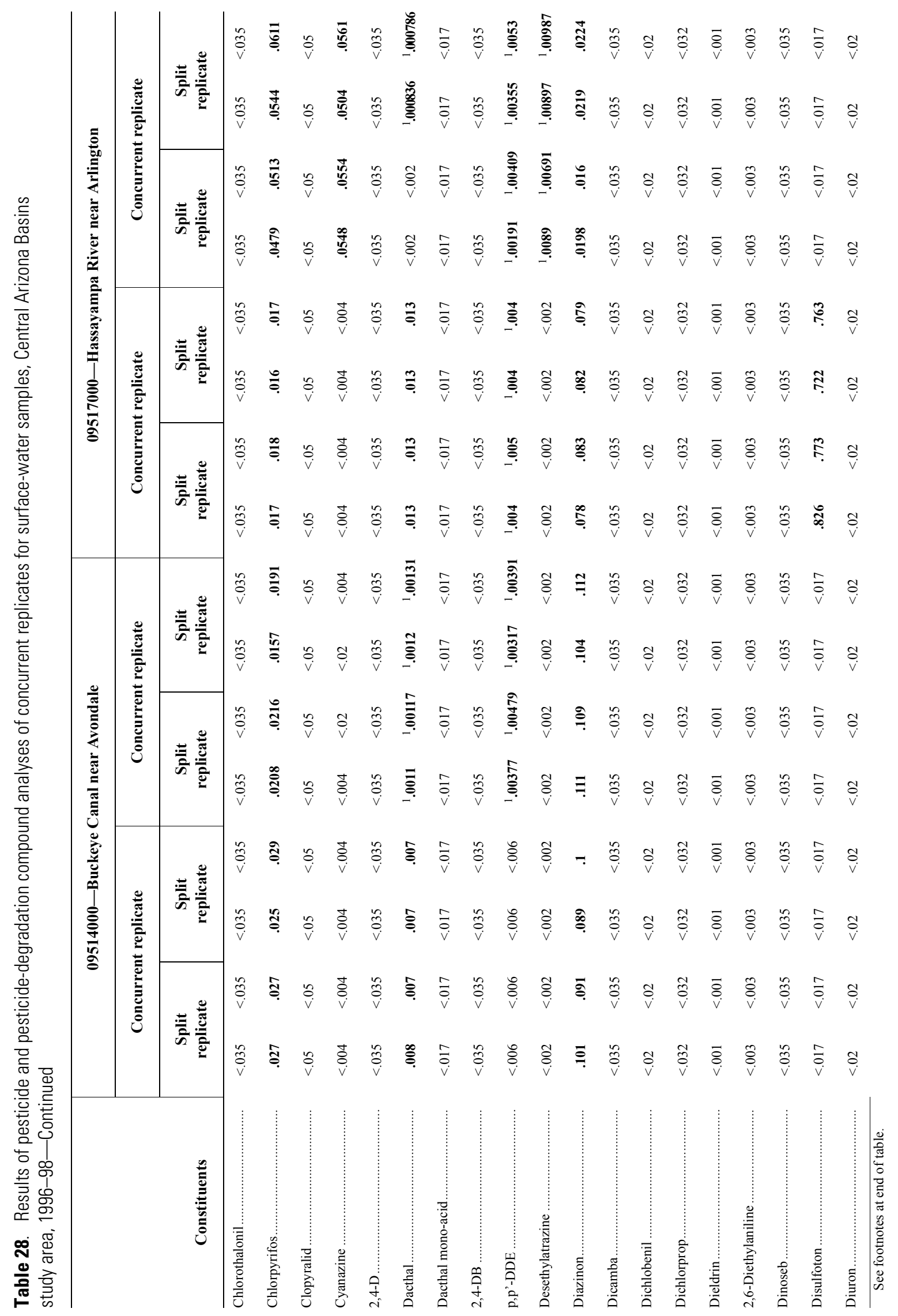




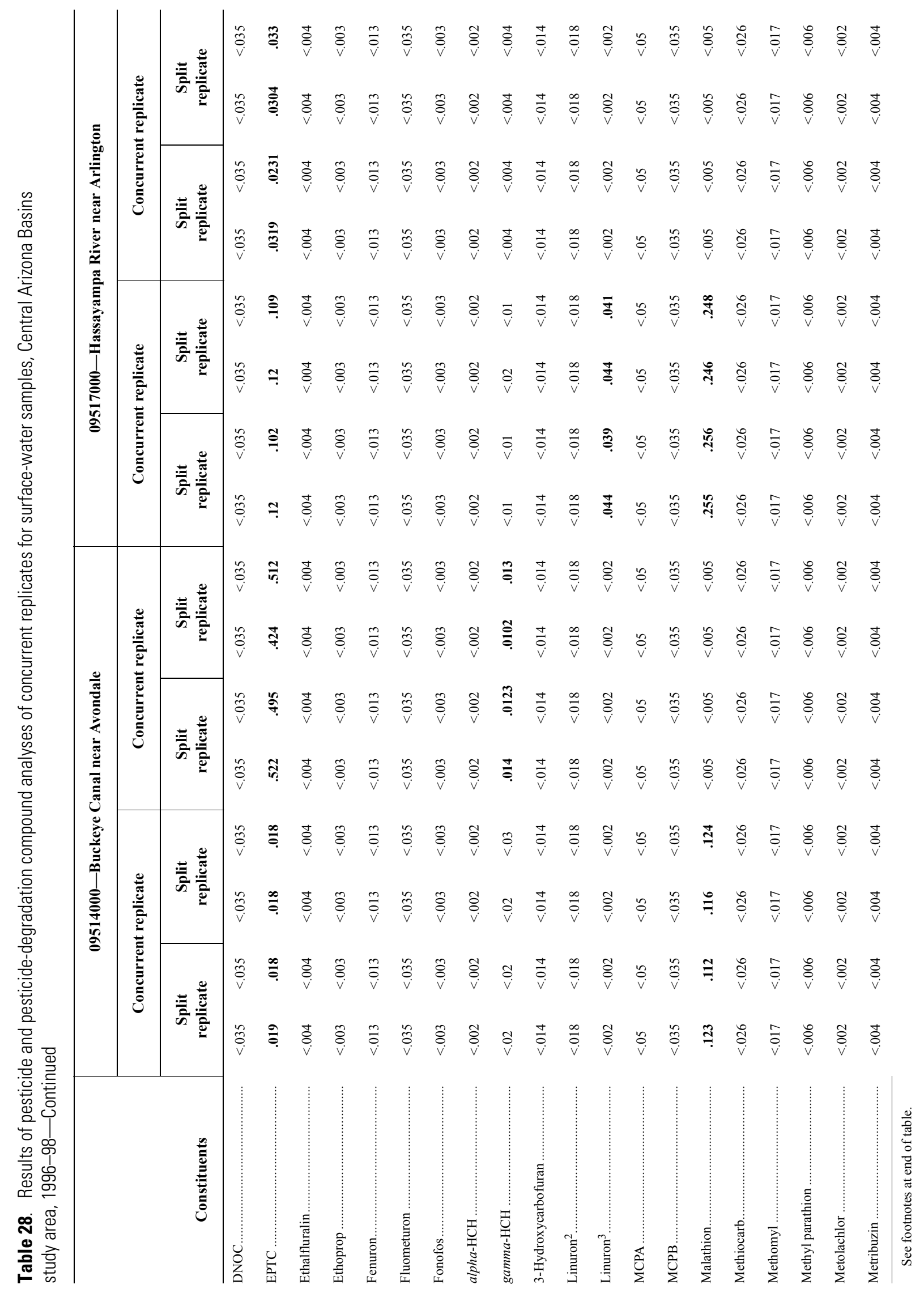

90 Characteristics of Shallow Deposits Beneath Rillito Creek, Pima County, Arizona 


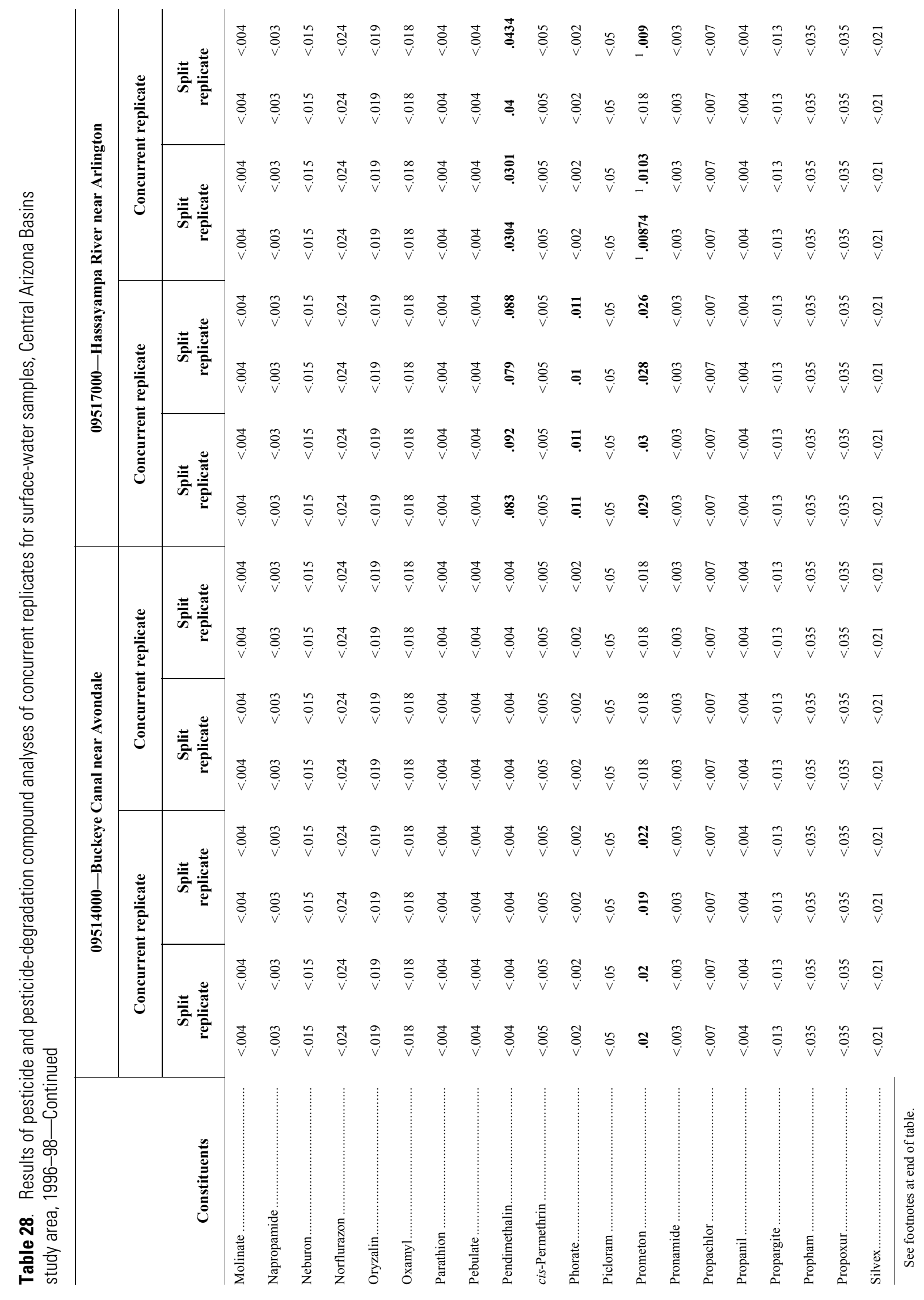




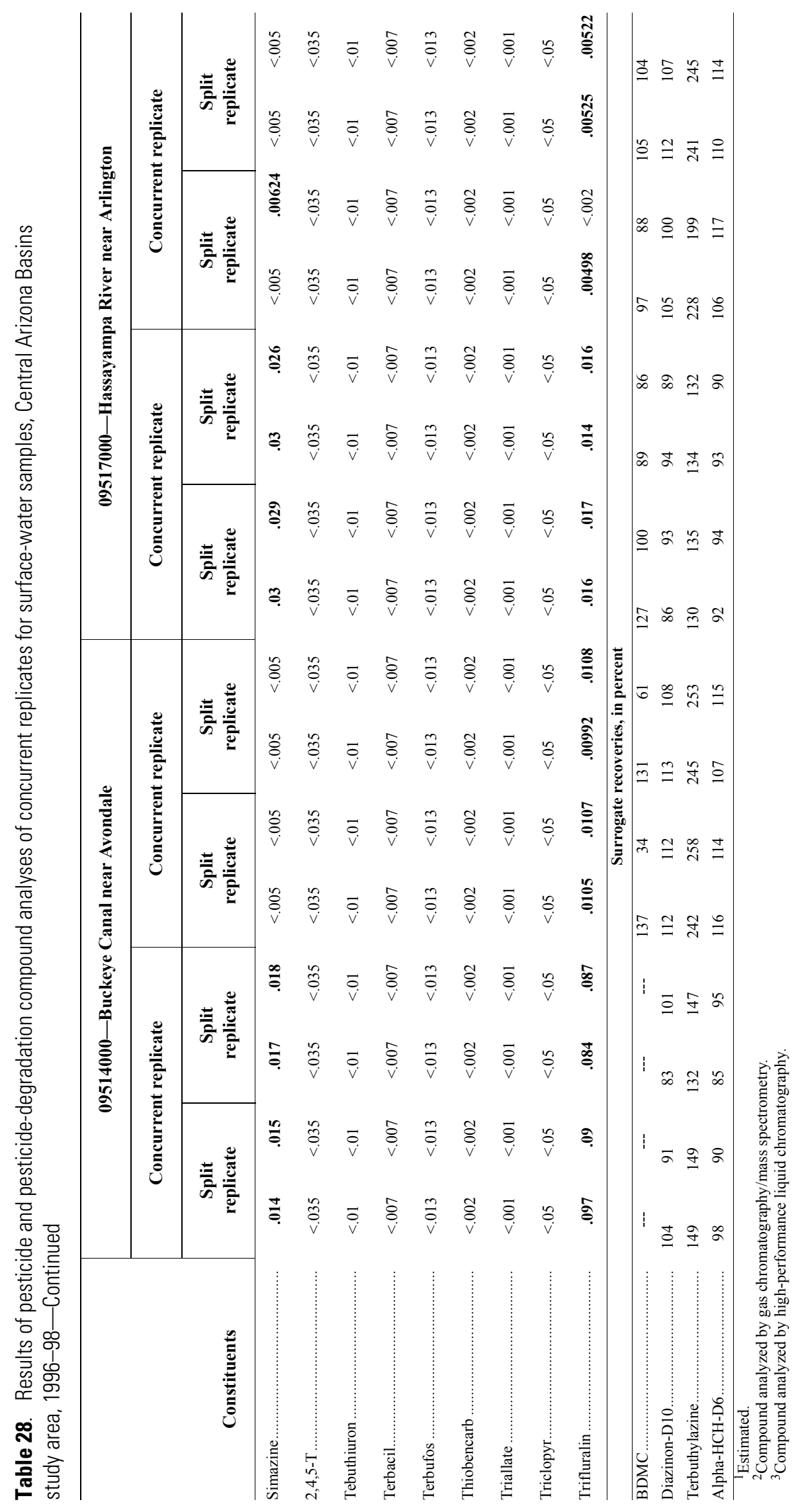

92 Characteristics of Shallow Deposits Beneath Rillito Creek, Pima County, Arizona 
Table 29. Pesticide and pesticide-degradation compounds detected in split-replicates for surface-water samples, Central Arizona Basins study area, 1996-98

[09514000, station number (see table 4). Concentrations are in micrograms per liter unless otherwise indicated. Sample dates and times in parentheses are for those compounds analyzed by high-performance liquid chromatography (see table 5). Bold type indicates results where the compound was present. Dashes indicate no data. <, less than]

\begin{tabular}{|c|c|c|c|c|c|c|c|c|}
\hline \multirow[b]{2}{*}{ Constituent } & \multicolumn{4}{|c|}{09514000 -Buckeye Canal near Avondale } & \multicolumn{4}{|c|}{09517000 - Hassayampa River near Arlington } \\
\hline & \multicolumn{2}{|c|}{$\begin{array}{c}\text { Split } \\
\text { replicate }\end{array}$} & \multicolumn{2}{|c|}{$\begin{array}{c}\text { Split } \\
\text { replicate }\end{array}$} & \multicolumn{2}{|c|}{$\begin{array}{c}\text { Split } \\
\text { replicate }\end{array}$} & \multicolumn{2}{|c|}{$\begin{array}{c}\text { Split } \\
\text { replicate }\end{array}$} \\
\hline Sample date & \multicolumn{2}{|c|}{$8-5-97$} & \multicolumn{2}{|c|}{$8-21-97$} & \multicolumn{2}{|c|}{$\begin{array}{c}8-4-97 \\
(8-19-97)\end{array}$} & \multicolumn{2}{|c|}{$2-18-98$} \\
\hline Sample time & 1000 & 1001 & 1140 & 1141 & $\begin{array}{c}1030 \\
(1120)\end{array}$ & $\begin{array}{c}1031 \\
(1121)\end{array}$ & 1410 & 1411 \\
\hline Acetochlor & $<0.002$ & $<0.002$ & $<0.002$ & --- & $<0.002$ & $<0.002$ & $<0.002$ & $<0.002$ \\
\hline Acifluorfen & $<.035$ & $<.035$ & $<.035$ & $<.035$ & $<.035$ & $<.035$ & $<.035$ & $<.035$ \\
\hline Alachlor & $<.002$ & $<.002$ & $<.002$ & --- & $<.002$ & $<.002$ & $<.002$ & $<.002$ \\
\hline Aldicarb & $<.016$ & $<.016$ & $<.016$ & $<.016$ & $<.016$ & $<.016$ & $<.55$ & $<.55$ \\
\hline Aldicarb sulfone & $<.016$ & $<.016$ & $<.016$ & $<.016$ & $<.016$ & $<.016$ & $<.1$ & $<.1$ \\
\hline Aldicarb sulfoxide & $<.021$ & $<.021$ & $<.021$ & $<.021$ & $<.021$ & $<.021$ & $<.021$ & $<.021$ \\
\hline Atrazine & .00566 & $<.001$ & $<.001$ & --- & .0151 & .0173 & $<.001$ & $<.001$ \\
\hline Azinphos-methyl & $<.001$ & $<.001$ & $<.001$ & --- & $<.001$ & $<.001$ & $<.001$ & $<.001$ \\
\hline Benfluralin & $<.002$ & $<.002$ & $<.002$ & --- & $<.002$ & $<.002$ & .0206 & .0232 \\
\hline Bentazon & $<.014$ & $<.014$ & $<.014$ & $<.014$ & $<.014$ & $<.014$ & $<.014$ & $<.014$ \\
\hline Bromacil & $<.035$ & $<.035$ & $<.035$ & $<.035$ & $<.035$ & $<.035$ & $<.035$ & $<.035$ \\
\hline Bromoxynil & $<.035$ & $<.035$ & $<.035$ & $<.035$ & $<.035$ & $<.035$ & $<.035$ & $<.035$ \\
\hline Butylate & $<.002$ & $<.002$ & $<.002$ & --- & $<.002$ & $<.002$ & $<.002$ & $<.002$ \\
\hline Carbaryl $^{2}$ & $<.008$ & $<.008$ & $<.008$ & $<.008$ & $<.008$ & $<.008$ & $<.008$ & $<.008$ \\
\hline Carbaryl $^{3}$ & $<.003$ & $<.003$ & $<.003$ & -- & $<.003$ & $<.003$ & ${ }^{1} .0522$ & ${ }^{1} .0556$ \\
\hline Carbofuran $^{2}$ & $<.028$ & $<.028$ & $<.028$ & $<.028$ & $<.028$ & $<.028$ & $<.12$ & $<.12$ \\
\hline Carbofuran $^{3}$ & $<.02$ & $<.003$ & $<.003$ & --- & $<.003$ & $<.003$ & $<.003$ & $<.003$ \\
\hline Chloramben & $<.011$ & $<.011$ & $<.011$ & $<.011$ & $<.011$ & $<.011$ & $<.42$ & $<.42$ \\
\hline Chlorothalonil & $<.035$ & $<.035$ & $<.035$ & $<.035$ & $<.035$ & $<.035$ & $<.48$ & $<.48$ \\
\hline Chlorpyrifos & .0105 & .0109 & .0176 & --- & .152 & .127 & .00678 & ${ }^{1} .00356$ \\
\hline Clopyralid & $<.05$ & $<.05$ & $<.05$ & $<.05$ & $<.05$ & $<.05$ & $<.23$ & $<.23$ \\
\hline Cyanazine & .0119 & .00886 & $<.004$ & -- & $<.004$ & .022 & $<.004$ & $<.004$ \\
\hline 2,4-D & $<.035$ & $<.035$ & $<.035$ & $<.035$ & $<.035$ & $<.035$ & $<.15$ & $<.15$ \\
\hline Dacthal & .00414 & ${ }^{1} .00381$ & ${ }^{1} .0194$ & --- & $<.002$ & $<.002$ & .0781 & .0812 \\
\hline Dacthal mono-acid & $<.017$ & $<.017$ & $<.017$ & $<.017$ & $<.017$ & $<.017$ & $<.017$ & $<.017$ \\
\hline 2,4-DB & $<.035$ & $<.035$ & $<.035$ & $<.035$ & $<.035$ & $<.035$ & $<.24$ & $<.24$ \\
\hline $\mathrm{p}, \mathrm{p}$ '-DDE & ${ }^{1} .00397$ & ${ }^{1} .00468$ & ${ }^{1} .004$ & -- & .0061 & ${ }^{1} .00379$ & .00796 & .00996 \\
\hline Deethylatrazine & $<.002$ & $<.002$ & $<.002$ & --- & ${ }^{1} .00659$ & ${ }^{1} .0056$ & $<.002$ & $<.002$ \\
\hline Diazinon & .0711 & .0665 & .0777 & -- & .0157 & .0226 & .0418 & .0501 \\
\hline Dicamba & $<.035$ & $<.035$ & $<.035$ & $<.035$ & $<.035$ & $<.035$ & $<.035$ & $<.035$ \\
\hline Dichlobenil & $<.02$ & $<.02$ & --- & $<.02$ & --- & $<.02$ & $<1.2$ & $<1.2$ \\
\hline Dichlorprop & $<.032$ & $<.032$ & $<.032$ & $<.032$ & $<.032$ & $<.032$ & $<.032$ & $<.032$ \\
\hline Dieldrin & $<.001$ & $<.001$ & $<.001$ & -- & $<.001$ & $<.001$ & $<.001$ & $<.001$ \\
\hline 2,6-Diethylaniline & $<.003$ & $<.003$ & $<.003$ & --- & $<.003$ & $<.003$ & $<.003$ & $<.003$ \\
\hline Dinoseb & $<.035$ & $<.035$ & $<.035$ & $<.035$ & $<.035$ & $<.035$ & $<.035$ & $<.035$ \\
\hline
\end{tabular}


Table 29. Pesticide and pesticide-degradation compounds detected in split-replicates for surface-water samples, Central Arizona Basins study area, 1996-98-Continued

\begin{tabular}{|c|c|c|c|c|c|c|c|c|}
\hline \multirow[b]{2}{*}{ Constituent } & \multicolumn{4}{|c|}{09514000} & \multicolumn{4}{|c|}{09517000} \\
\hline & \multicolumn{2}{|c|}{$\begin{array}{c}\text { Split } \\
\text { replicate }\end{array}$} & \multicolumn{2}{|c|}{$\begin{array}{c}\text { Split } \\
\text { replicate }\end{array}$} & \multicolumn{2}{|c|}{$\begin{array}{l}\text { Split } \\
\text { replicate }\end{array}$} & \multicolumn{2}{|c|}{$\begin{array}{c}\text { Split } \\
\text { replicate }\end{array}$} \\
\hline Disulfoton & $<.017$ & $<.017$ & $<.017$ & --- & $<.017$ & $<.017$ & $<.017$ & $<.017$ \\
\hline Diuron & $<0.02$ & $<0.02$ & --- & $<0.02$ & --- & $<0.02$ & ${ }^{1} 7.61$ & ${ }^{1} 7.4$ \\
\hline DNOC & $<.035$ & $<.035$ & $<.035$ & $<.035$ & $<.035$ & $<.035$ & $<.42$ & $<.42$ \\
\hline EPTC & $<.002$ & $<.002$ & $<.002$ & --- & $<.002$ & ${ }^{1} .00239$ & $<.002$ & $<.002$ \\
\hline Ethalfluralin & $<.004$ & $<.004$ & $<.004$ & --- & $<.004$ & $<.004$ & $<.004$ & $<.004$ \\
\hline Ethoprop & $<.003$ & $<.003$ & --- & --- & $<.003$ & $<.003$ & $<.003$ & $<.003$ \\
\hline Fenuron & $<.013$ & $<.013$ & $<.013$ & $<.013$ & $<.013$ & $<.013$ & $<.013$ & $<.013$ \\
\hline Fluometuron & $<.035$ & $<.035$ & $<.035$ & $<.035$ & $<.035$ & $<.035$ & $<.035$ & $<.035$ \\
\hline Fonofos & $<.003$ & $<.003$ & $<.003$ & --- & $<.003$ & $<.003$ & $<.003$ & $<.003$ \\
\hline alpha-HCH & $<.002$ & $<.002$ & $<.002$ & --- & $<.002$ & $<.002$ & $<.002$ & $<.002$ \\
\hline gamma-HCH & .0158 & .0133 & .0194 & --- & $<.004$ & $<.004$ & $<.004$ & $<.004$ \\
\hline 3-Hydroxycarbofuran & $<.014$ & $<.014$ & $<.014$ & $<.014$ & $<.014$ & $<.014$ & $<.014$ & $<.014$ \\
\hline Linuron $^{2}$ & $<.018$ & $<.018$ & $<.018$ & $<.018$ & $<.018$ & $<.018$ & $<.018$ & $<.018$ \\
\hline Linuron $^{3}$ & $<.002$ & $<.002$ & $<.002$ & --- & $<.002$ & $<.002$ & $<.02$ & $<.02$ \\
\hline MCPA & $<.05$ & $<.05$ & $<.05$ & $<.05$ & $<.05$ & $<.05$ & $<.17$ & $<.17$ \\
\hline MCPB & $<.035$ & $<.035$ & $<.035$ & $<.035$ & $<.035$ & $<.035$ & $<.14$ & $<.14$ \\
\hline Malathion & $<.005$ & $<.005$ & $<.005$ & --- & $<.005$ & $<.005$ & $<.005$ & $<.005$ \\
\hline Methiocarb & $<.026$ & $<.026$ & $<.026$ & $<.026$ & $<.026$ & $<.026$ & $<.026$ & $<.026$ \\
\hline Methomyl & $<.017$ & $<.017$ & $<.017$ & $<.017$ & $<.017$ & $<.017$ & $<.017$ & $<.017$ \\
\hline Methyl parathion & $<.006$ & $<.006$ & $<.006$ & --- & .0253 & .0354 & $<.006$ & $<.006$ \\
\hline Metolachlor & $<.002$ & $<.002$ & --- & --- & $<.002$ & $<.002$ & $<.002$ & $<.002$ \\
\hline Metribuzin & $<.004$ & $<.004$ & $<.004$ & --- & $<.004$ & $<.004$ & $<.01$ & $<.004$ \\
\hline Molinate & $<.004$ & $<.004$ & $<.004$ & --- & $<.004$ & $<.004$ & $<.004$ & $<.004$ \\
\hline Napropamide & $<.003$ & $<.003$ & $<.003$ & --- & $<.003$ & $<.003$ & $<.003$ & $<.003$ \\
\hline Neburon & $<.015$ & $<.015$ & $<.015$ & $<.015$ & $<.015$ & $<.015$ & $<.015$ & $<.015$ \\
\hline Norflurazon & $<.024$ & $<.024$ & $<.024$ & $<.024$ & $<.024$ & $<.024$ & $<.024$ & $<.024$ \\
\hline Oryzalin & $<.019$ & $<.019$ & --- & $<.019$ & --- & $<.019$ & $<.31$ & $<.31$ \\
\hline Oxamyl & $<.018$ & $<.018$ & $<.018$ & $<.018$ & $<.018$ & $<.018$ & $<.018$ & $<.018$ \\
\hline Parathion & $<.004$ & $<.004$ & $<.004$ & --- & $<.004$ & $<.004$ & $<.004$ & $<.004$ \\
\hline Pebulate & $<.004$ & $<.004$ & $<.004$ & --- & $<.004$ & $<.004$ & $<.004$ & $<.004$ \\
\hline Pendimethalin & $<.004$ & $<.004$ & $<.004$ & --- & .017 & .0136 & .0357 & .0375 \\
\hline cis-Permethrin & $<.005$ & $<.005$ & $<.005$ & --- & $<.005$ & $<.005$ & $<.005$ & $<.005$ \\
\hline Phorate & $<.002$ & $<.002$ & $<.002$ & --- & $<.002$ & $<.002$ & $<.002$ & $<.002$ \\
\hline Picloram & $<.05$ & $<.05$ & $<.05$ & $<.05$ & $<.05$ & $<.05$ & $<.05$ & $<.05$ \\
\hline Prometon & ${ }^{1} .0178$ & $<.018$ & $<.018$ & --- & $<.018$ & ${ }^{1} .0102$ & ${ }^{1} .00849$ & ${ }^{1} .00753$ \\
\hline Pronamide & $<.003$ & $<.003$ & $<.003$ & --- & $<.003$ & $<.003$ & $<.003$ & $<.003$ \\
\hline Propachlor & $<.007$ & $<.007$ & $<.007$ & --- & $<.007$ & $<.007$ & $<.007$ & $<.007$ \\
\hline Propanil & $<.004$ & $<.004$ & --- & --- & $<.004$ & $<.004$ & $<.004$ & $<.004$ \\
\hline Propargite & $<.013$ & $<.013$ & $<.013$ & --- & $<.013$ & $<.013$ & $<.013$ & $<.013$ \\
\hline
\end{tabular}


Table 29. Pesticide and pesticide-degradation compounds detected in split-replicates for surface-water samples, Central Arizona Basins study area, 1996-98-Continued

\begin{tabular}{|c|c|c|c|c|c|c|c|c|}
\hline \multirow[b]{2}{*}{ Constituent } & \multicolumn{4}{|c|}{09514000} & \multicolumn{4}{|c|}{09517000} \\
\hline & \multicolumn{2}{|c|}{$\begin{array}{c}\text { Split } \\
\text { replicate }\end{array}$} & \multicolumn{2}{|c|}{$\begin{array}{c}\text { Split } \\
\text { replicate }\end{array}$} & \multicolumn{2}{|c|}{$\begin{array}{c}\text { Split } \\
\text { replicate }\end{array}$} & \multicolumn{2}{|c|}{$\begin{array}{c}\text { Split } \\
\text { replicate }\end{array}$} \\
\hline Propham & $<.035$ & $<.035$ & $<.035$ & $<.035$ & $<.035$ & $<.035$ & $<.035$ & $<.035$ \\
\hline Propoxur & $<.035$ & $<.035$ & $<.035$ & $<.035$ & $<.035$ & $<.035$ & $<.035$ & $<.035$ \\
\hline Silvex & $<0.021$ & $<0.021$ & $<0.021$ & $<0.021$ & $<0.021$ & $<0.021$ & $<0.021$ & $<0.021$ \\
\hline Simazine & .0123 & $<.005$ & $<.01$ & --- & $<.005$ & .00835 & .0152 & .0149 \\
\hline $\begin{array}{l}\text { (2,4,5-trichlorophenoxy) } \\
\text { acetic acid }\end{array}$ & $<.035$ & $<.035$ & $<.035$ & $<.035$ & $<.035$ & $<.035$ & $<.035$ & $<.035$ \\
\hline Tebuthiuron & $<.01$ & $<.01$ & $<.01$ & --- & $<.01$ & $<.01$ & $<.01$ & $<.01$ \\
\hline Terbacil & $<.007$ & $<.007$ & $<.007$ & --- & $<.007$ & $<.007$ & $<.007$ & $<.007$ \\
\hline Terbufos & $<.013$ & $<.013$ & $<.013$ & --- & $<.013$ & $<.013$ & $<.013$ & $<.013$ \\
\hline Thiobencarb & $<.002$ & $<.002$ & --- & --- & $<.002$ & $<.002$ & $<.002$ & $<.002$ \\
\hline Triallate & $<.001$ & $<.001$ & --- & --- & $<.001$ & $<.001$ & $<.001$ & $<.001$ \\
\hline Triclopyr & $<.05$ & $<.05$ & $<.05$ & $<.05$ & $<.05$ & $<.05$ & $<.25$ & $<.25$ \\
\hline Trifluralin & .00704 & .00684 & ${ }^{2} .00329$ & --- & .0043 & .00448 & .000265 & ${ }^{2} .00295$ \\
\hline \multicolumn{9}{|c|}{ Surrogate recoveries, in percent } \\
\hline BDMC & 56 & 56 & 57 & 45 & --- & 65 & 81 & 90 \\
\hline Diazinon-D1 & 98 & 93 & 101 & --- & 97 & 99 & 90 & 95 \\
\hline Terbuthylazine & 198 & 191 & 146 & --- & E 214 & 203 & 115 & 132 \\
\hline Alpha-HCH-D6 & 97 & 100 & 104 & --- & 198 & 97 & 100 & 98 \\
\hline
\end{tabular}

${ }^{1}$ Compound analyzed by gas chromatography/mass spectrometry.
${ }^{2}$ Estimated. 


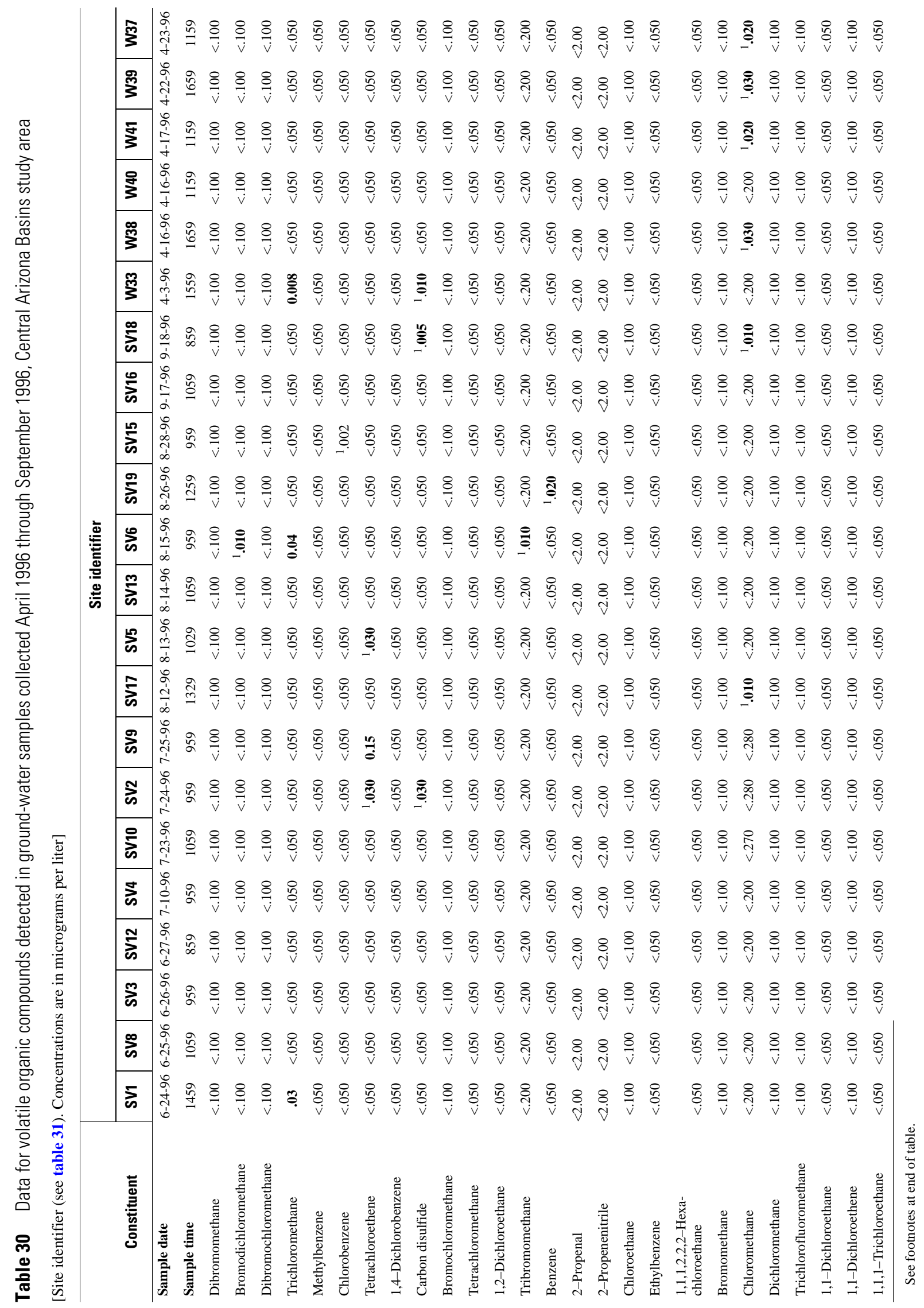




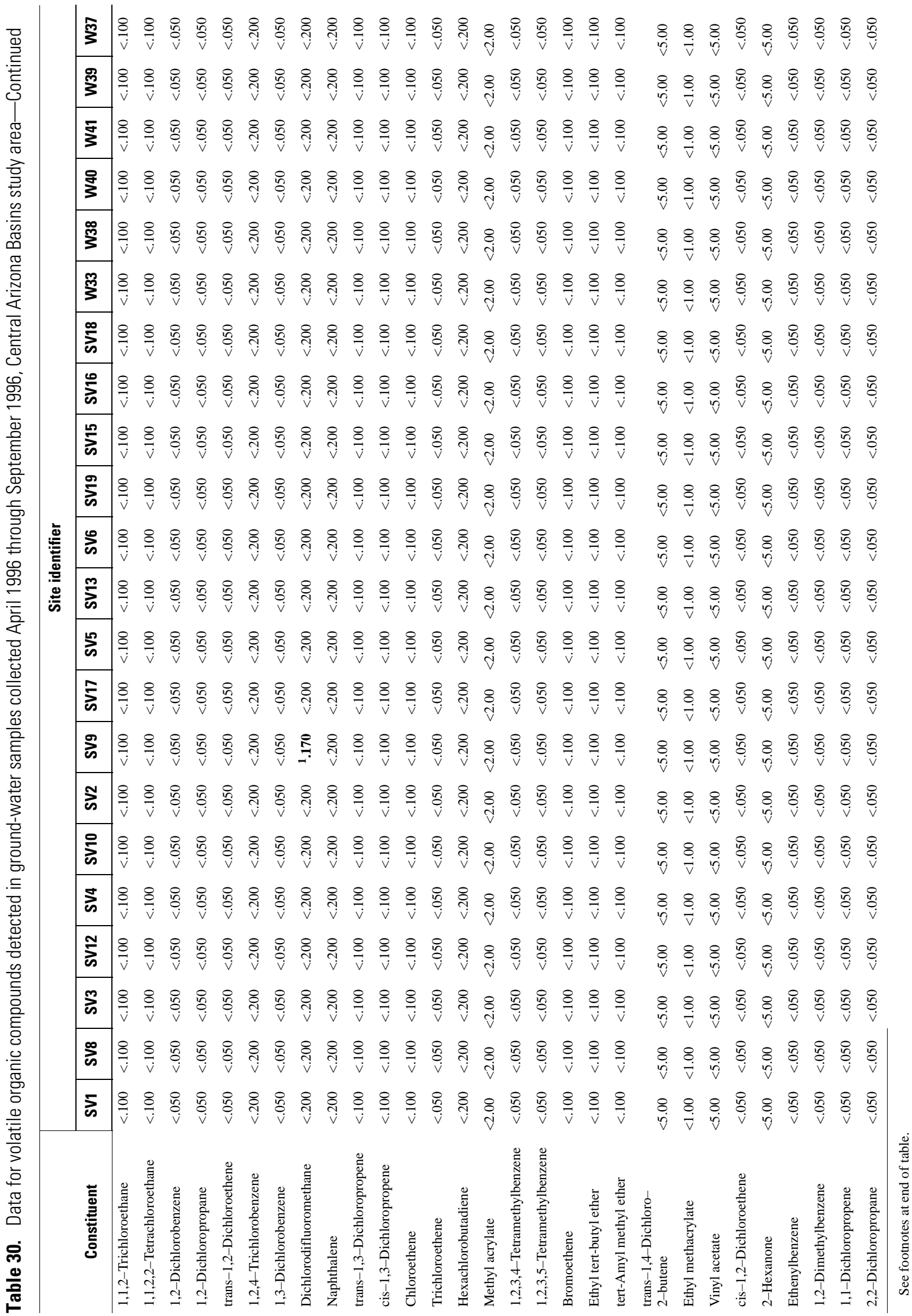




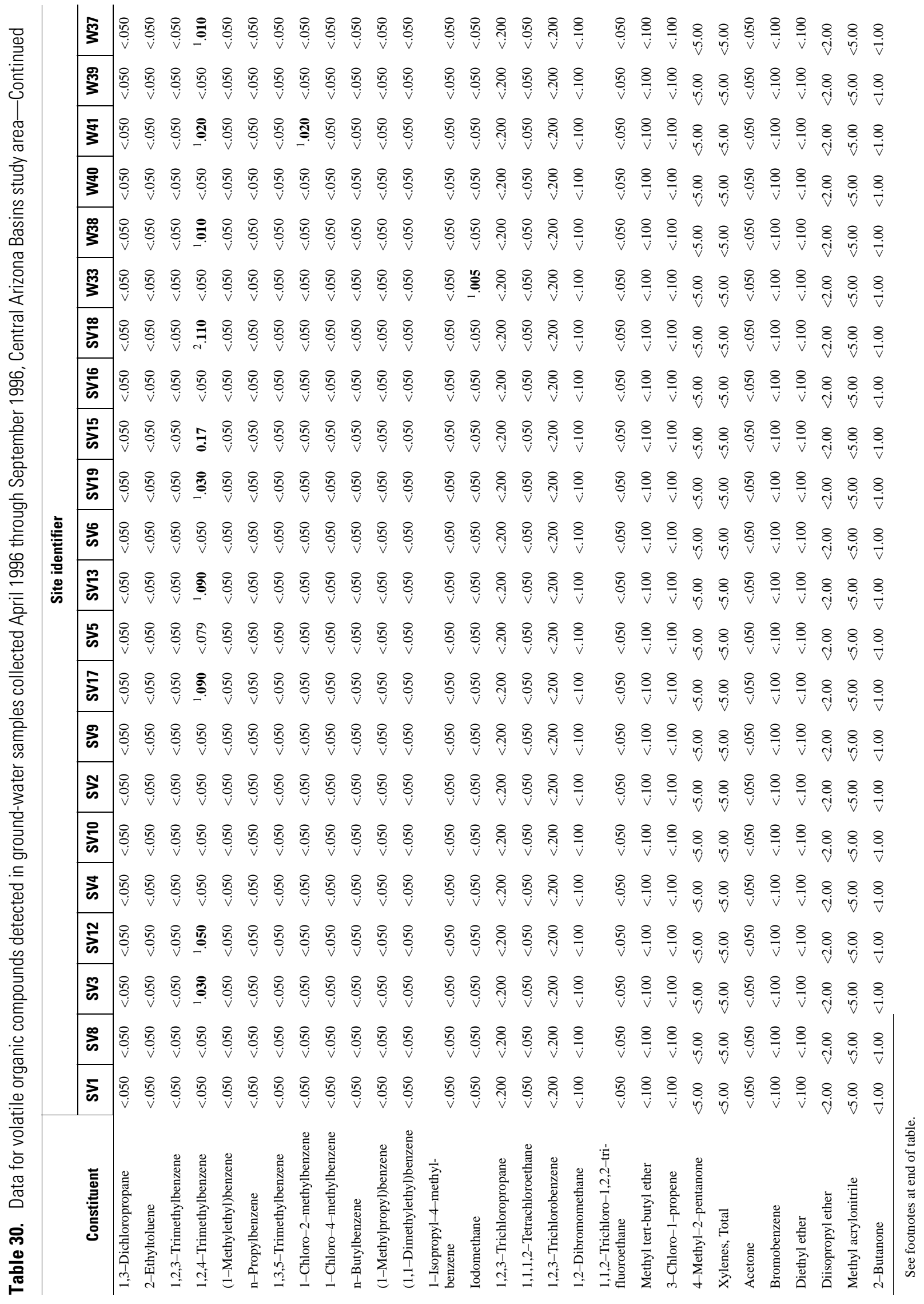




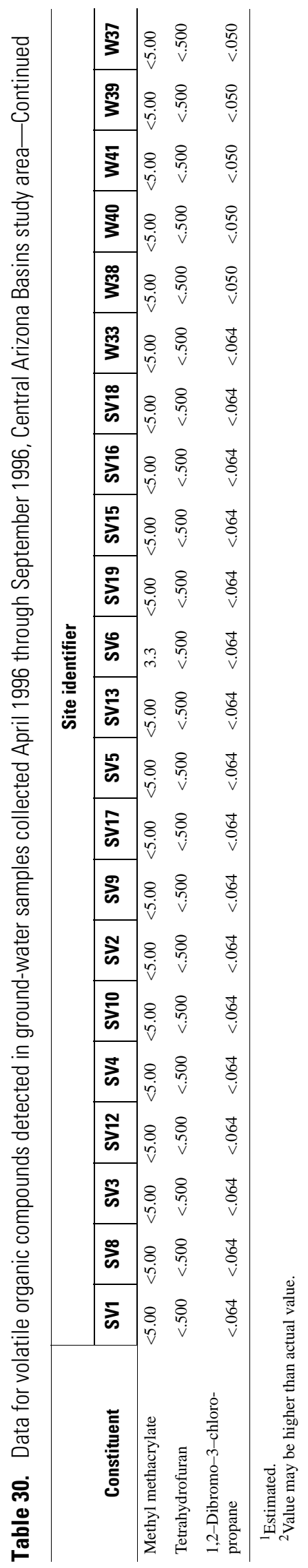

100 Characteristics of Shallow Deposits Beneath Rillito Creek, Pima County, Arizona 
Table 31. Site information for wells sampled, Central Arizona Basins study area, 1996-98

[unsurv, unsurveyed]

\begin{tabular}{|c|c|c|c|c|c|c|c|}
\hline $\begin{array}{c}\text { Site } \\
\text { identifier }\end{array}$ & Well number & $\begin{array}{c}\text { Site } \\
\text { identifier }\end{array}$ & Well number & $\begin{array}{c}\text { Site } \\
\text { identifier }\end{array}$ & Well number & $\begin{array}{c}\text { Site } \\
\text { identifier }\end{array}$ & Well number \\
\hline \multicolumn{8}{|c|}{ Sierra Vista subbasin sub-unit survey } \\
\hline SV1 & (D-23-22)31dda & SV6 & (D-21-21)33bda & SV11 & (D-20-22) $16 \mathrm{ddb}$ & SV16 & (D-17-21)29dca \\
\hline SV2 & (D-23-20)01acc2 & SV7 & (D-21-23)33aаa & SV12 & (D-19-18)33aаa2 & SV17 & (D-17-19) $17 \mathrm{ddd} 2$ \\
\hline SV3 & (D-23-23)06bcc2 & SV8 & $\begin{array}{l}\text { (D-21-19) 06cc } \\
\text { unsurv }\end{array}$ & SV13 & (D-19-22)27acc & SV18 & (D-17-20) $18 b b b$ \\
\hline SV4 & (D-22-21)33aаa & SV9 & $(\mathrm{D}-20-20) 32 \mathrm{dcb} 2$ & SV14 & (D-18-23) 32abc & SV19 & (D-15-20)21bda \\
\hline SV5 & (D-22-18) 13bbd & SV10 & $(\mathrm{D}-20-20) 18 \mathrm{ccc}$ & SV15 & (D-18-21)33bbb & & \\
\hline \multicolumn{8}{|c|}{ Upper Santa Cruz Basin sub-unit survey } \\
\hline $\mathrm{SC} 1$ & (D-10-14)06dca & SC9 & (D-15-14) $02 d d c$ & SC16 & (D-17-14)21bba & $\mathrm{SC} 23$ & (D-19-13)22ddd \\
\hline $\mathrm{SC} 2$ & (D-11-13)34add & SC10 & (D-15-16)06aad & SC17 & (D-17-15)09bac & $\mathrm{SC} 24$ & (D-21-13) 19cdb \\
\hline $\mathrm{SC} 3$ & (D-11-14) 10dab2 & $\mathrm{SC} 11$ & (D-15-16)34cba & SC18 & (D-17-15)23add & $\mathrm{SC} 25$ & (D-21-13)30cda \\
\hline $\mathrm{SC} 4$ & (D-12-12)01cda & $\mathrm{SC} 12$ & (D-16-14)06cdc & SC19 & (D-18-16)01bcc & SC26 & (D-22-13)09cad \\
\hline SC5 & (D-13-13) 18acb & $\mathrm{SC} 13$ & (D-16-14) $11 \mathrm{bca}$ & $\mathrm{SC} 20$ & (D-19-12)36cbb & $\mathrm{SC} 27$ & (D-23-14)26cca \\
\hline SC6 & (D-13-13) $18 \mathrm{cbd}$ & SC14 & (D-16-16)04dab & $\mathrm{SC} 21$ & (D-19-13) 07cba & SC28 & (D-23-14)30baa \\
\hline SC7 & (D-13-14)35aad3 & $\mathrm{SC} 15$ & (D-17-13) $11 \mathrm{dcd} 2$ & $\mathrm{SC} 22$ & (D-19-13)22ccc & $\mathrm{SC} 29$ & (D-23-15)31cbb \\
\hline $\mathrm{SC} 8$ & (D-14-13)23aca & & & & & & \\
\hline
\end{tabular}

Agricultural land-use study

\begin{tabular}{llllll}
\hline AG1 & $(\mathrm{B}-01-02) 14 \mathrm{cac}$ & AG4 & $(\mathrm{B}-01-03) 34 \mathrm{cdd}$ & AG7 & (C-01-03)07bbd \\
AG2 & $(\mathrm{B}-01-02) 32 \mathrm{cbb}$ & AG5 & $(\mathrm{B}-01-04) 33 \mathrm{cad}$ & AG8 & (C-01-04)04caa \\
AG3 & $(\mathrm{B}-01-03) 25 \mathrm{ccb}$ & AG6 & $(\mathrm{C}-01-03) 02 \mathrm{dcc}$ & AG9 & (C-01-04)20baa
\end{tabular}

West Salt River Valley sub-unit survey

\begin{tabular}{|c|c|c|c|c|c|c|c|}
\hline W10 & (A-01-01)14bab2 & W20 & (A-03-02)16aaa & W30 & (B-02-01)01bbb & W40 & (B-05-03)24ada \\
\hline W11 & $(\mathrm{A}-01-01) 22 \mathrm{dcc}$ & W21 & $(\mathrm{A}-03-02) 26 \mathrm{dcb}$ & W31 & (B-02-01)36add & W41 & (B-06-03)36ddd \\
\hline W12 & $(\mathrm{A}-01-01) 28 \mathrm{bba}$ & W22 & (A-04-01)05acc & W32 & (B-02-02)28bca & W42 & $(\mathrm{C}-01-02) 19 \mathrm{ccc}$ \\
\hline W13 & $(\mathrm{A}-01-02) 21 \mathrm{bcd}$ & W23 & (A-05-02)35acb2 & W33 & (B-02-02)33ada & W43 & (C-01-04)20bab \\
\hline W14 & (A-01-02)34ada & W24 & (B-01-01)14dbd & W34 & (B-03-01)02dcc & W44 & (D-01-02)10aca \\
\hline W15 & $(\mathrm{A}-02-02) 36 \mathrm{cba}$ & W25 & (B-01-02)09cbd & W35 & (B-03-01)09ccc & ${ }^{1} \mathrm{~W} 45$ & (B-01-02)22dba2 \\
\hline W16 & $(\mathrm{A}-02-03) 13 \mathrm{dac} 2$ & W26 & (B-01-02)32add & W36 & (B-03-01)34bbb & ${ }^{1} \mathrm{~W} 47$ & (C-01-03)05bbd \\
\hline W17 & $(\mathrm{A}-02-04) 21 \mathrm{ccb}$ & W27 & (B-01-03)13dba & W37 & (B-04-01)07aaa & ${ }^{1} \mathrm{~W} 61$ & (B-01-04)32dbb \\
\hline W18 & (A-03-01)25abb & W28 & (B-01-03)30cdc & W38 & (B-04-03)04bdb & ${ }^{1} \mathrm{~W} 63$ & (B-02-02)24baa \\
\hline W19 & (A-03-01)34ddd2 & W29 & (B-01-03)34bbb & W39 & (B-05-03)15aaa & ${ }^{1} \mathrm{~W} 65$ & $(\mathrm{C}-01-02) 06 \mathrm{dbb}$ \\
\hline
\end{tabular}

\footnotetext{
${ }^{1}$ Well was not used for interpretation of sub-unit survey results
} 